Linköping Studies in Science and Technology

Dissertations, No.1600

\title{
Logistics Service Providers Going Green - A Framework for Developing Green Service Offerings
}

\author{
Karin Isaksson
}

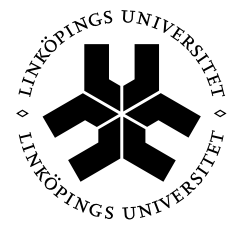

\section{Linköping University \\ INSTITUTE OF TECHNOLOGY}

2014

Department of Management and Engineering Linköpings universitet, SE-581 83 Linköping, Sweden 
(C) Karin Isaksson "Unless otherwise noted"

Logistics Service Providers Going Green - A Framework for Developing Green Service Offerings.

Linköping Studies in Science and Technology, Dissertations, No. 1600

ISBN: 978-91-7519-315-1

ISSN: 0345-7524

Printed by: LiU-Tryck, Linköping

Distributed by:

Linköping University

Department of Management and Engineering

SE-581 83 Linköping, Sweden

Tel: +46 13281000 


\section{Abstract}

Environmental impact has increasingly become a "buzzword" and an important topic. This topic has been integrated into the agenda of many companies worldwide, and this dissertation focuses on the transportation and logistics industry. Environmental concerns have gained increased attention among many logistic service providers (LSPs) due to the environmental impact from their operations, and they have been identified of having a significant role in reducing the environmental burden in the supply chain. An environmental approach of the LSPs' business has also been identified as a way to achieve competitive advantage and provide market opportunities where the development and marketing of new products and services associated with green issues are suggested as important aspects for future growth. However, considering the scarcity of research regarding this topic, a study that reveals potential aspects in the development of green service offerings can bridge the knowledge gap and provide opportunities for further research within this field. The purpose of this dissertation is therefore to develop and explain a framework for LSPs' development of green service offerings. The purpose is addressed by first investigating LSPs' service development from a general perspective in order to, in a second stage, reach a better understanding of the implications when integrating green aspects in LSPs' service development efforts.

Theoretically, this dissertation departed from service marketing literature or more specifically new service development (NSD) research. This resulted in a conceptual framework including key dimensions and aspects regarding a company's NSD efforts and activities. From this foundation, the theoretical framework was developed further based on research regarding LSPs' service development and innovation management. Finally the framework was extended with green logistics literature as well as research regarding LSPs' green development and influences on their service offerings.

Empirically, this research is mainly based on qualitative data from an in-depth case study on a large LSP active on the Swedish market. In addition, empirical data from a multiple case study and a questionnaire survey conducted for the Licentiate thesis were used in order to enrich the analysis regarding the LSPs' development of green service offerings. The analysis followed a stepwise approach where literature and empirical data were analysed.

One of the main results in this dissertation is the framework for LSPs' new service development, consisting of five dimensions: NSD culture, NSD strategy, NSD process focus, IT use and expertise and NSD knowledge and skills. The NSD framework presents a holistic view of the LSPs' NSD efforts by revealing different dimensions, their roles and relations to each other as well as the pre-requisites to take into consideration in the development of new services. Thus, the different NSD dimensions should not solely be viewed as isolated dimensions; instead, there is a need for LSPs to have a holistic view and understanding of the NSD activities' reciprocity.

Another main result concerns the adaption of the NSD framework to green service development. The results reveal some pre-requisites relevant for LSPs to consider in their efforts to develop green service offerings and are summarised in the following main dimensions: 
- Creating green awareness in the NSD culture - encourage participation regarding green initiatives within the organisation, defining a "common picture" in order to facilitate collaboration efforts and knowledge exchange concerning green expertise. The support from top management was also identified of having an influencing impact.

- Defining the strategic approach of green service offerings - integrate a green concern in the overall business strategy and to define the strategic role and incentives for developing green service offerings. The results also suggest LSPs to adapt green NSD efforts to different business contexts and market possibilities to match existing resources and skills with customers' green requirements, and to perform a segmentation of customers' environmental work and ambitions to increase the understanding of customers' green attitudes and requirements.

- Create processes and routines to facilitate spreading of green knowledge highlights the relevance of a process focus for spreading green knowledge both from an external and internal perspective. It involves e.g. adoption of certifications, procedures for environmental calculations and documentation as well as routines to spread and integrate green knowledge among employees as well as identification of customers' green requirements.

- Improve green internal knowledge and build green collaborations - provide training and education to increase the level of green awareness and knowledge among employees as well as customers and strive for collaboration efforts both internally and externally to utilise each other's knowledge and resources towards the development of green service offerings.

- Increase transparency of green information both internally and externally - improve green information transparency to build both internal and external trust and increase possibilities to effectively use other actors' knowledge and resources to develop environmental improvements in the supply chain. Integration of IT expertise and synchronisations of IT systems to facilitate and support environmental work and development of green service offerings. 


\section{Sammanfattning}

Miljöpåverkan har i en allt större utsträckning blivit ett nyckelord och en viktig punkt på många företags agendor. Denna avhandling tar sig an detta område inom transport- och logistikindustrin. Miljöfrågan har fått ökad uppmärksamhet bland många logistikföretag på grund av miljöpåverkan från deras aktiviteter och verksamhet men logistikföretagen har också blivit uppmärksammande då de spelar en viktig roll att minska miljöpåverkan i hela försörjningskedjan. Att arbete aktivt med sin miljöpåverkan har också uppmärksammats som ett sätt att uppnå konkurrensfördelar samt öppna upp för nya marknadsmöjligheter. $\mathrm{T}$ ex kan utveckling och lansering av nya produkter och tjänster som associeras med ett miljötänk komma att utgöra en viktig del i företagens fortsatta utveckling och tillväxt. Dock råder det en brist på forskning kring detta område. Därför kan en studie som belyser olika aspekter vid utveckling av miljötjänster bland logistikföretag vara av värde för att tillföra ökad kunskap samt öppna upp för fortsatta forskningsmöjligheter kring detta område. Syftet med denna avhandling är att utveckla ett ramverk för logistikföretags utveckling av miljötjänster samt förklara dess innehåll. Syftet besvaras genom att först undersöka logistikföretags generella tjänsteutveckling för att på så vis erhålla en djupare förståelse för att i nästa steg undersöka vad detta får för implikationer vid integrering av miljöaspekter i tjänsteutvecklingen.

Teoretiskt tog avhandlingen avstamp i litteratur om tjänstemarknadsföring eller mer specifikt i forskning kring ny tjänsteutveckling. Det resulterade i ett konceptuellt ramverk kring nyckeldimensioner och aspekter som kan kopplas till företags utveckling av nya tjänster. Utifrån detta utvecklades det teoretiska ramverket vidare med forskning kring logistikföretags tjänste- och innovationsutveckling. Slutligen utökades det teoretiska ramverket med litteratur om miljölogistik samt forskning kring logistikföretags miljöutveckling och dess påverkan på deras tjänsteutbud.

Empirin byggde i huvudsak på en kvalitativ fallstudie, där ett större logistikföretag verksam på den svenska marknaden undersöktes. Dessutom har även empiri erhållen från flerfallstudien samt enkätstudien i Licentiatavhandling använts för att berika analysen kring logistikföretags utveckling av miljötjänster. Analysen följde en fler-stegs ansats där både litteratur och empirisk data analyserades.

Ett av huvudresultaten i avhandlingen är det framtagna ramverket för logistikföretags tjänsteutveckling, bestående av fem olika dimensioner: kultur, strategi, process fokus, ITanvändning och -expertis samt kunskaper och förmågor. Ramverket ämnar ge en holistisk bild av logistikföretags tjänsteutveckling genom att identifiera viktiga dimensioner, deras roll samt relationer till varandra såväl som förutsättningar som är relevanta att ta $\mathrm{i}$ beaktning vid utveckling av nya tjänster. Detta medför att de olika dimensionerna inte enbart ska ses som isolerade enheter utan att det finns ett behov för logistikföretag att ha ett helhetsperspektiv kring tjänsteutvecklingen samt förståelse kring hur deras aktiviteter samverkar.

Ett annat huvudresultat berör anpassningen av ramverket till utvecklandet av miljötjänster. Analysen ledde till en rad olika förutsättningar vilka är relevanta för logistikföretag att ta $i$ beaktning vid utvecklandet av miljötjänster, och resultatet har summerats i följande dimensioner nedan: 
- Skapa miljömedvetenhet $i$ tjänsteutvecklingskulturen - uppmuntra deltagande kring miljöåtgärder i organisationen, samt definiera en "gemensam bild" för att underlätta samarbeten och kunskapsutbyte kring miljöinitiativ. Ledningens stöd identifierades också som en viktig punkt i denna utveckling.

- Definiera den strategiska ståndpunkten kring miljötjänster - integrera miljöaspekter i den övergripande affärsstrategin samt definiera den strategiska rollen och incitamenten för utveckling av miljötjänster. Resultaten stöder att logistikföretag ska anpassa tjänsteutvecklingsåtgärder till olika affärsområden och marknadsmöjligheter för att matcha existerande resurser och förmågor med kunders miljökrav. Resultaten föreslår genomförandet av segmentering av kunders miljöarbete och ambitioner för att öka förståelsen kring kunders miljöattityder samt krav.

- Skapa processer och rutiner för spridning av miljökunskap - denna dimension poängterar vikten av att ha ett process fokus kring spridning av miljökunskap både ur ett internt och ur ett externt perspektiv. Detta inkluderar bland annat införande av certifieringar, tillvägagångssätt för miljöberäkningar och dokumentation så väl som rutiner för att sprida och integrera miljökunskap hos anställda, samt identifiering av kunders miljökrav.

- Förbättra miljökunskap internt samt bilda miljösamarbeten - tillhandahålla utbildning för att höja medvetandet och kunskapsnivå kring miljö hos anställda och kunder samt sträva efter samarbeten både internt och externt för att ta del av varandras kunskaper och resurser vid utvecklandet av miljötjänster.

- Öka transparensen av miljöinformation både internt och externt - förbättra transparensen kring miljöinformation för att bygga förtroende både internt och externt samt öka möjligheterna att effektivt ta del av andra aktörers kunskaper och resurser för att utveckla miljöbättringar i försörjningskedjan. Integrering av IT-expertis samt synkronisering av IT-system för att underlätta och stödja miljöarbetet och utveckling av miljötjänster. 


\section{Acknowledgements}

I was looking for a challenge and I found it: the challenge to become a $\mathrm{PhD}$. It has been a great and inspiring time but it has not been completed without sacrifices. However, like someone special once said to me: things don't grow if you don't bless them with your patience. So, I did. Weeks became months and months became years until today, when I finally am writing some of the last words of my dissertation. These words may be the last but also the most emotional, since these words are showing my gratitude to those people who have supported me during my $\mathrm{PhD}$ time.

First, I would like to express my gratitude to my supervisors Maria Huge-Brodin, Maria Björklund and Mats Abrahamsson whose encouragement and support from the first start to the final end have enabled me to develop an understanding of my research subject.

I am also thankful to Gunnar Stefansson who read an earlier draft of this dissertation and made many valuable comments and suggestions.

Another thank you is directed to the Ragnar Söderberg foundation, for funding the research project, The development of Logistics companies towards environmentally sustainable strategies, which I have been a part of.

In addition, I would like to thank my colleagues at the division Logistics Management as well as at the division Industrial Economics and a special thank you to Kristina Dalberg for your general support during my $\mathrm{PhD}$ time.

Lastly, I offer my deepest gratitude and blessings to Stephan and to my family, which during the last year has expanded with another beloved family member. Words are not enough to thank you for helping me to find the strength and patience of carrying on with this process, and for that I am eternally grateful.

So, this challenge has now come to an end and it is time for me to find a new challenge. Wish me good luck!

Linköping, April 2014

Karin Helena Isaksson 


\section{Table of Content}

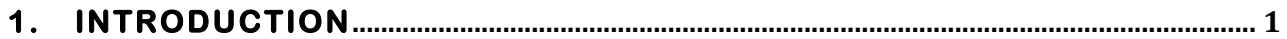

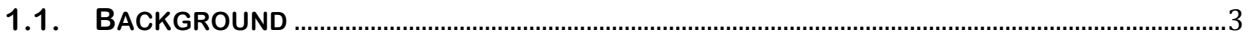

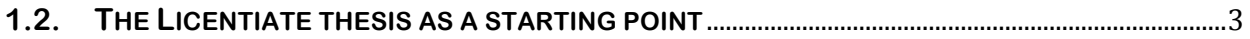

1.2.1 Main conclusions from the Licentiate Thesis.......................................................... 4

1.2.2 The connection between the Licentiate thesis and the Dissertation ............. 5

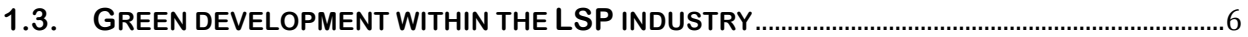

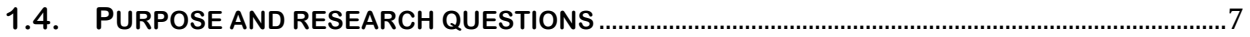

1.4.1 Service development within the LSP industry..................................................... 8

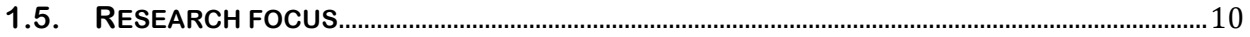

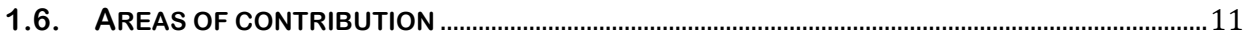

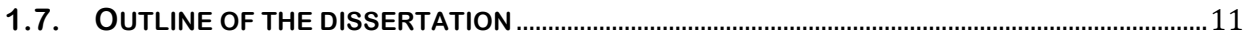

2. METHODOLOGY

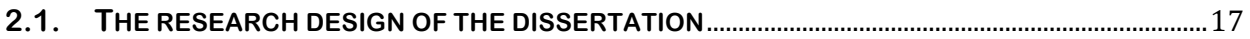

2.2. IDENTIFYING AND DEFINING THE RESEARCH PROBLEM

2.2.1 The methodological link to the Licentiate thesis..............................................18

2.2.2 The researcher's philosophical views and personal experience...................19

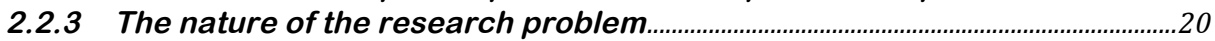

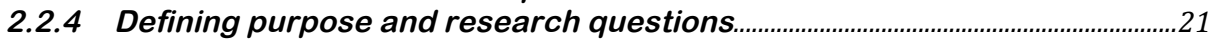

2.3. THEORETICAL PERSPECTIVE OF THIS DISSERTATION........................................................... 22

2.3.1 The aim, content and link between the different literature parts....................23

2.3.2 Search methods for the different literature areas..................................................24

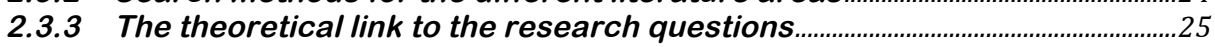

2.4. EMPIRICAL INPUT TO THE DISSERTATION ..........................................................................25

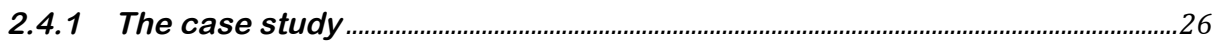

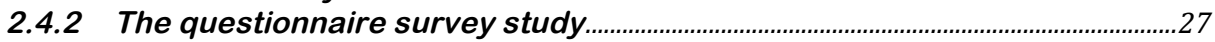

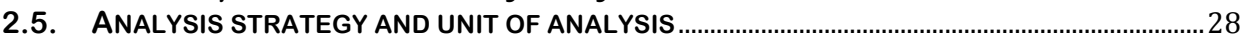

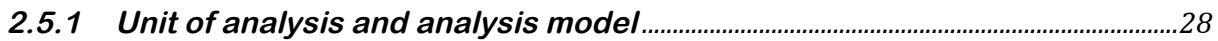

2.5.2 The analysis strategy for the case and its link to the RQs..............................29

2.6. REFLECTIONS UPON CONTRIBUTIONS OF THE DISSERTATION............................................ 30

3. SERVICE DEVELOPMENT MANAGEMENT - A THEORETICAL FRAMEWORK

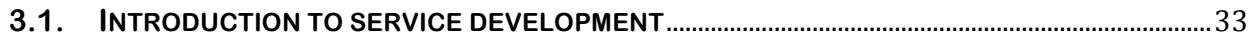

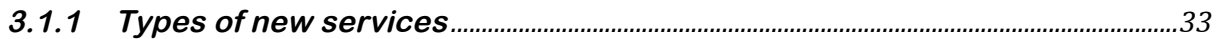

3.1.2 Why a strategic perspective on NSD?

3.2. TOWARDS A FRAMEWORK FOR NSD

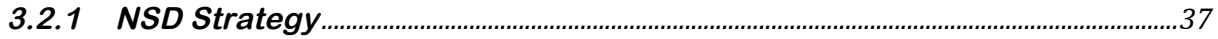

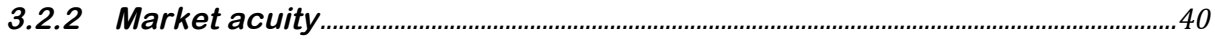

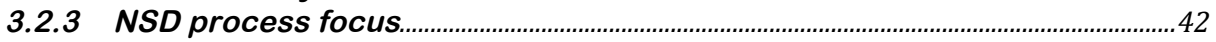

3.2.4 IT Expertise

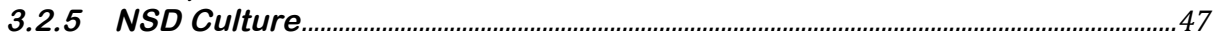

3.2.6 A conceptual framework for management of NSD.............................................49

4. THE LSP CONTEXT OF NEW SERVICE DEVELOPMENT ................................53

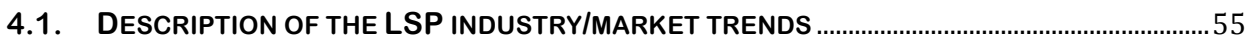

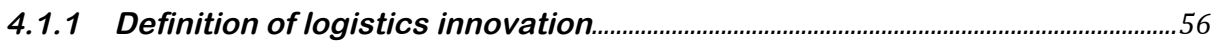

4.2. THE STATUS OF AND NEED FOR NSD WITHIN THE LOGISTICS INDUSTRY...........................56

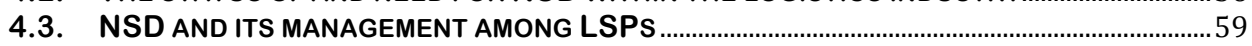

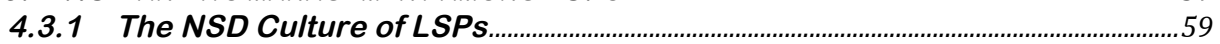

4.3.2 Strategic focus and existence of an NSD Strategy ............................................61

4.3.3 Adoption of NSD processes and structures...........................................................64 
4.3.4 The multifaceted role of information technology ................................................69

4.3.5 Knowledge and skills needed for LSPs' NSD.

4.4. TOWARDS A FRAMEWORK FOR LSPS' MANAGEMENT OF NSD …......................................... 75

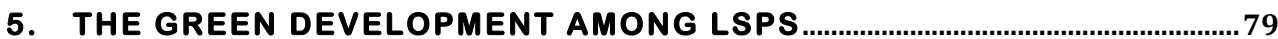

5.1. GREEN LOGISTICS - A NEW AREA OF NSD AMONG LSPS ............................................. 81

5.1.1 Drivers and barriers towards adoption of green initiatives...............................82

5.2. ASPECTS INFLUENCING THE DEVELOPMENT OF GREEN LOGISTICS SERVICES..................86

5.3. TOWARDS A FRAMEWORK FOR LSPS' MANAGEMENT OF GREEN NSD ................................ 92

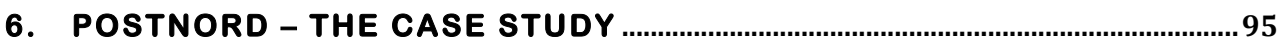

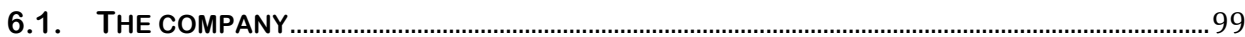

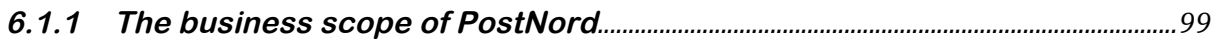

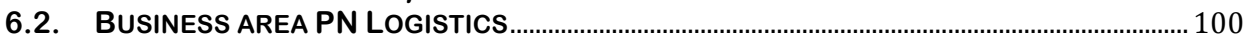

6.2.1 The overall strategic development and business model of PN Logistics......

6.2.2 The range of service offerings and position on the market........................... 101

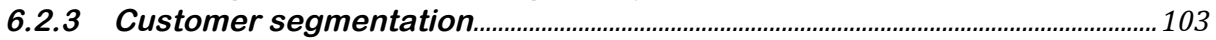

6.3. SERVICE DEVELOPMENT MANAGEMENT WITHIN PN LOGISTICS - FROM AN

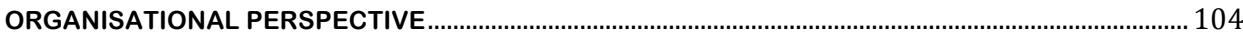

6.3.1 Collaboration between the different operational units .....................................105

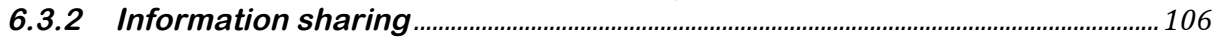

6.4. SERVICE DEVELOPMENT MANAGEMENT WITHIN PN LOGISTICS - FROM A STRATEGIC

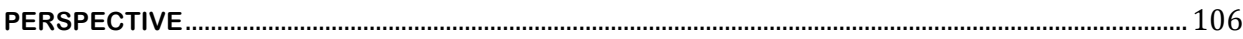

6.4.1 Requirements when developing new service offerings................................... 108

6.4.2 Identifying new ideas, evaluating and closing down existing service

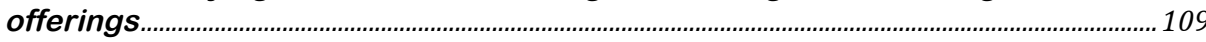

6.5. SERVICE DEVELOPMENT MANAGEMENT WITHIN PN LOGISTICS - FROM A PROCESS

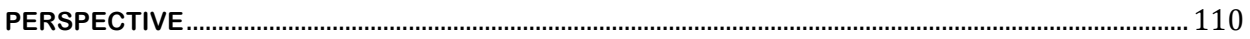

6.5.1 Existence of structured procedures and supporting tools............................111

6.5.2 From idea generation to launching of the service concept.............................112

6.6. SERVICE DEVELOPMENT MANAGEMENT WITHIN PN LOGISTICS - FROM AN IT

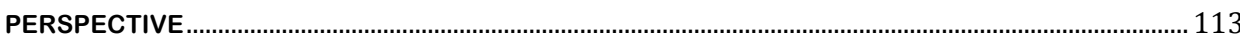

6.7. SERVICE DEVELOPMENT MANAGEMENT WITHIN PN LOGISTICS - FROM A MARKET

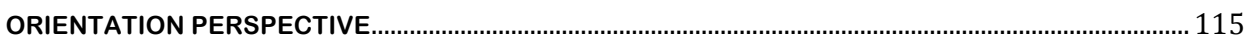

6.7.1 Market analysis as an input to service development.......................................115

6.7.2 Identifying new customers and monitoring the competitors..........................115

6.7.3 External actors as an inspiration source for service development............116

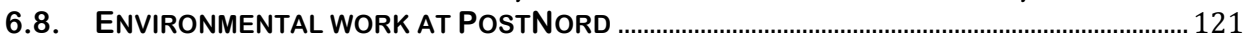

6.8.1 Environmental initiatives adopted within PostNord.........................................122

6.8.2 Driving forces regarding the environmental work .......................................... 123

6.8.3 Barriers regarding the environmental work

6.8.4 Environmental concerns - a potential competitive advantage...................... 124

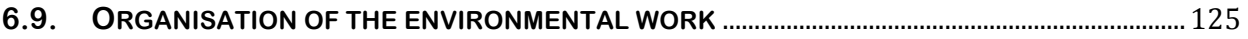

6.10. GREEN SERVICE OFFERINGS AND CUSTOMERS' GREEN REQUIREMENTS ......................126

6.10.1 Environmental work and green service offerings within PN Logistics.126

6.10.2 Efforts for bringing the business areas together and developing green

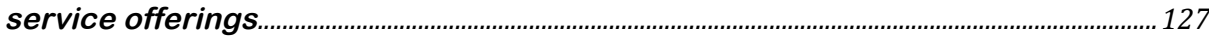

6.10.3 Customer segmentation based on environmental requirements.............. 128

6.10.4 Communication regarding environmental efforts to customers................129

6.10.5 External inputs for the environmental work

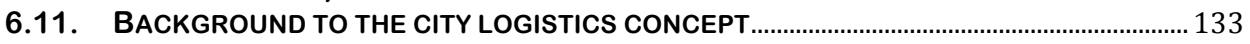

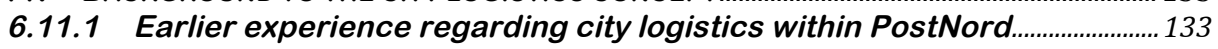

6.11.2 Identified problems within the city logistics area.............................................134

6.11.3 The next step within the city logistics concept.............................................. 135 
7.1. THE ANALYSIS APPROACH

7.2. ANALYSIS I - IDENTIFYING PRE-REQUISITES FOR \& ROLES OF THE NSD DIMENSIONS 141

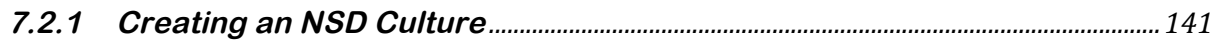

7.2.2 A strategic focus of NSD and existence of an NSD strategy......................... 145

7.2.3 A focus on processes and structural relevance of NSD ................................... 149

7.2.4 The IT adoption and expertise regarding NSD ................................................152

7.2.5 Internal and external knowledge and skills regarding NSD .............................153

7.3. ANALYSIS II - LINKING THE DIFFERENT NSD DIMENSIONS TO EACH OTHER ................... 158

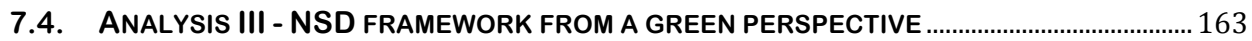

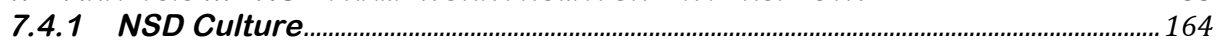

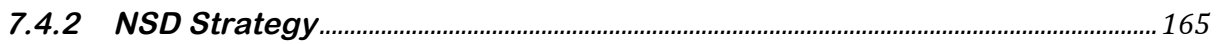

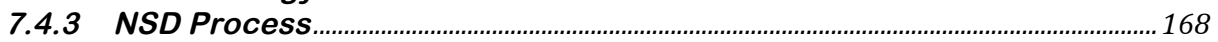

7.4.4 NSD Knowledge and Skills......................................................................................... 170

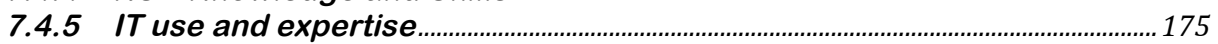

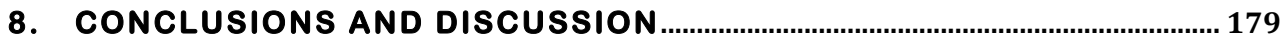

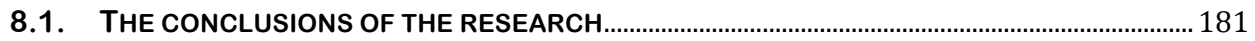

8.1.1 The LSP NSD framework

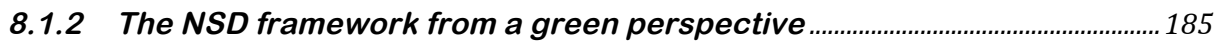

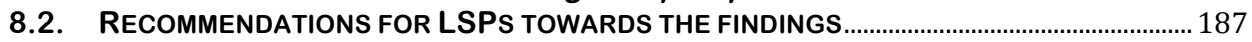

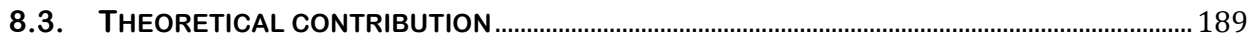

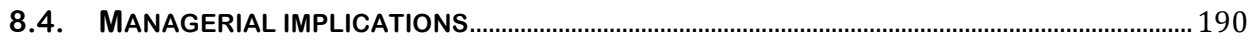

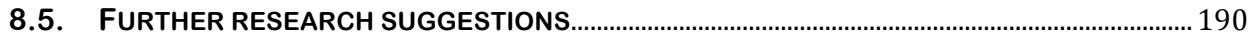

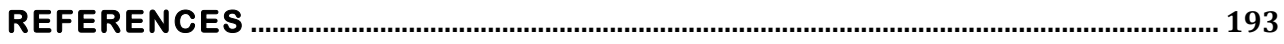

\section{APPENDIX}

Licentiate Thesis: Logistics Service Providers going green - insights from the Swedish market

\section{LIST OF FIGURES}

Figure 1.1 The structure of the Licentiate thesis..........................................................................

Figure 1.2 The main focus of the dissertation......................................................................................

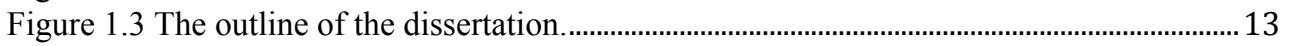

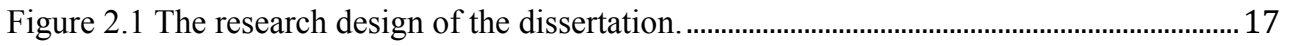

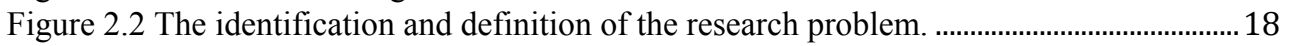

Figure 2.3 The theoretical and empirical contents of the Licentiate thesis.................................. 18

Figure 2.4 The theoretical perspectives of the dissertation .............................................................. 23

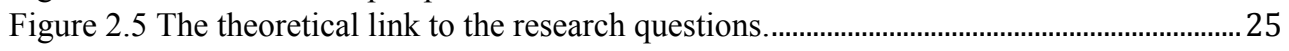

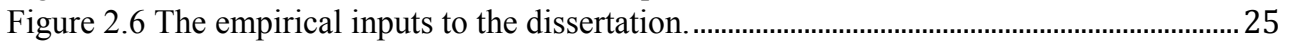

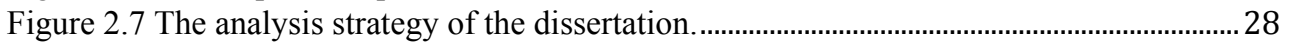

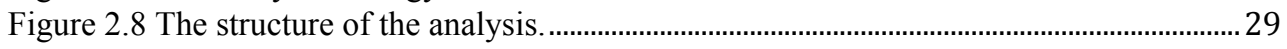

Figure 3.1 Conceptual model of NSD competence dimensions (source: Menor \& Roth, 2007,

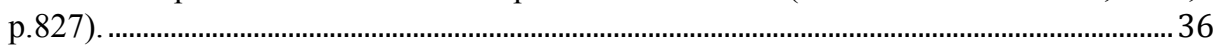

Figure 3.2 The NSD Process Cycle (source: Johnson et al., 2000, p. 18)..................................... 43

Figure 3.3 A conceptual framework of NSD management. .......................................................... 51

Figure 4.1 Matrix of Market Leadership Examples and Key Dynamics (source: Flowers et al.,

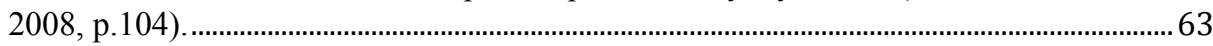

Figure 4.2 A Logistics Innovation Process Model (source: Flint et al., 2005, p.127)............... 65

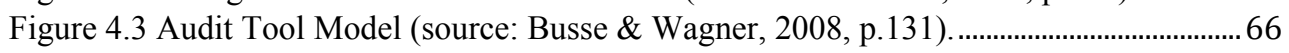

Figure 4.4 A platform and toolkit model (source: Wagner \& Franklin, 2008, p. 71)................67 
Figure 4.5 Perceptions of 3PLs IT capabilities (source: Third-Party Logistics Study 2013, p.

21).

Figure 4.6 Examples of customer clue gathering activities (source: Flint et al., 2005, p. 133).

Figure 4.7 The empirical guide for an LSP's management of NSD.....................

Figure 5.1 Framework towards green logistics services (source: Isaksson, 2012, first paper in

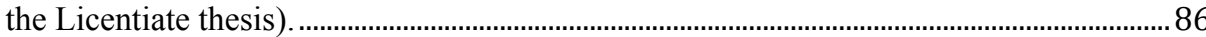

Figure 5.2 The empirical guide for a LSP's management of green NSD.................................... 93

Figure 6.1 PostNord's different business areas (source: PostNord Annual Report, 2012)...... 99

Figure 6.2 Structural organisation of PN Logistics business area (source: PostNord)........... 100

Figure 6.3 The Business Model of PN Logistics (source: PostNord Annual report, 2012). . 101

Figure 6.4 PN Logistics' range of service offerings (source: PostNord, 2012)......................... 102

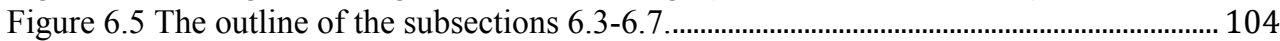

Figure 6.6 The structure of the functional unit Market and Sales (source: PostNord, 2012).104

Figure 6.7 The Business process of PN Logistics (source: PostNord, 2012)........................... 111

Figure 7.1 The analysis approach................................................................................................ 141

Figure 7.2 The link between NSD culture and NSD strategic focus.......................................... 158

Figure 7.3 NSD strategy, NSD culture and NSD process........................................................... 160

Figure 7.4 NSD culture, NSD strategy, NSD process and NSD knowledge and skills......... 161

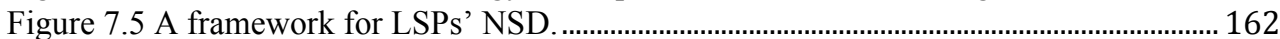

Figure 7.6 The developed NSD framework.................................................................................. 164

Figure 8.1 The NSD framework for LSPs.................................................................................. 181

\section{LIST OF TABLES}

Table 1 The repondents contributions to the case descrpition.......................................................27

Table 2 Summarisation of the NSD Strategy dimension. ............................................................... 40

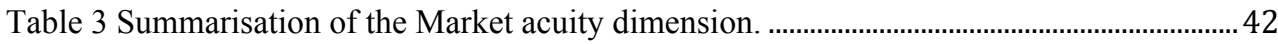

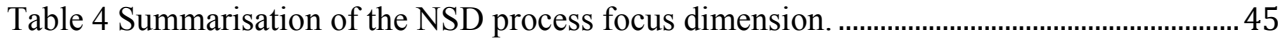

Table 5 The summarisation of the dimension IT Expertise. ............................................................ 47

Table 6 Summarisation of the NSD Culture dimension.................................................................... 49

Table 7 Transport related measures adopted by LSP to reduce the environmental impact...... 81

Table 8 Supply chain related measures adopted by LSPs to reduce the environmental impact.

Table 9 Examples of drivers towards the adoption of green initiatives (source Isaksson, 2012,

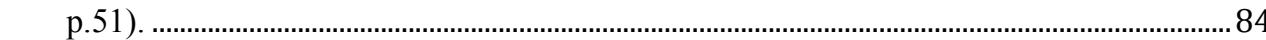

82

Table 10 Examples of different functions involved in managing green initiatives....................8 88

Table 11 Examples of ICT aplications for managing the environmental impact........................89

Table 12 Examples of arguments for investing in ICT to support green initiatives. ................. 90

Table 13 The level of ICT integration with other supply chain participants................................90 



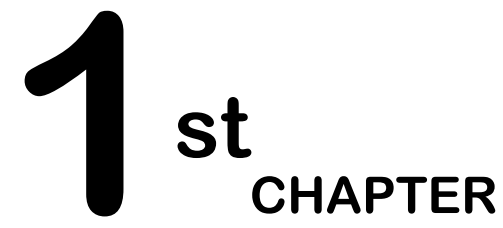

\section{INTRODUCTION}

This chapter aims to first introduce the focus area of this dissertation, namely the development of green service offerings among logistics service providers (LSPS). Thereafter follows a section describing the main results of the Licentiate thesis and its connection to this dissertation. This description together with a review of service development within the LSP industry as well as its connection to environmental management leads thereafter to the purpose and the research questions of this dissertation. In addition, the research focus as well as the practical and academic relevance of this study is also discussed and the chapter ends with a short description of the outline of this dissertation. 
$-2-$ 


\subsection{Background}

Environmental impact has increasingly become a "buzzword" and an important topic that has been integrated into the agenda of many companies. This growing concern emanates from various sources such as increasing awareness and pressures from society and customers regarding reducing the environmental impact as well as potential economic benefits that may be perceived. As a response, many companies have started to develop sustainable solutions in order to reduce the environmental burden from their activities both within the company as well as in the supply chain. The development towards sustainable supply chains together with the fact that logistics outsourcing continues to be a growing business (Third-Party Logistics Study, 2012) has led to an increased focus on logistics service providers (LSPs) who have been identified as important actors in this process (Rossi et al., 2013; Zailani et al., 2011), not only due to the fact that LSPs' core activities (e.g. transport activities) have a strong environmental impact but also because LSPs have the opportunities and capabilities to provide transport and supply chain solutions that take environmental considerations into account.

The greening of LSPs' business has also been identified as a source for achieving and maintaining competitive advantage (Lieb \& Lieb, 2010) and provides market possibilities where the development of new products and services associated with environmental concerns is considered to be an important aspect for the future growth of these firms. For example, in a report, the CEO of Deutsche Post DHL states the following regarding the focus on sustainability logistics:

“...it is important for us - so important that providing sustainability solutions has been integrated into our corporate strategy ("Strategy 2015"). We are also confident that this will increase our - and our customers' - competiveness."(DHL, 2010, p. 10)

This implies that in order to successfully take advantage of such new market opportunities, LSPs need to place a higher focus on environmental concerns as well as an increased focus on how these concerns can be integrated into their business operations and service offerings. This dissertation focuses on LSPs and on the challenge of integrating environmental concerns into these actors' service development efforts. But to address this new challenge, an increased understanding of why LSPs choose to adopt green initiatives is needed. This was investigated in a previously presented Licentiate thesis, a summary of whose results is presented below, which in turn contributes to the background and the basis of this dissertation.

\subsection{The Licentiate thesis as a starting point}

The content and approach of this dissertation takes its starting point in the Licentiate thesis Logistics Service Providers going green - insights from the Swedish market (which can be found in its complete version in Appendix I). The Licentiate thesis takes an LSP perspective and its content and structure is illustrated in Figure 1.1 below. The focus was to describe and understand how different factors can affect the adoption of green initiatives among LSPs. The term "factor" was divided into triggers, drivers and barriers as well as characteristics of the firm. In addition, the Licentiate thesis also aimed to describe how the adoption of green initiatives can be reflected in the service offering (as illustrated by the large arrow in Figure 1.1 below). 


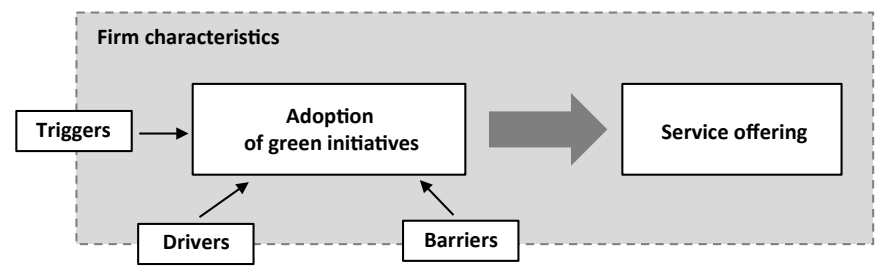

Figure 1.1 The structure of the Licentiate thesis.

The next section gives a summary of some of the main contributions from the Licentiate thesis.

\subsubsection{Main conclusions from the Licentiate Thesis}

The findings from the Licentiate thesis suggest that adoption of green initiatives among the studied LSPs should not be viewed as a short-lived trend nor as an established practice; instead, it can be described as a potential business opportunity necessary to maintain longterm competitiveness.

As indicated above, in the long term, adopting green initiatives may create an advantage of having superior standing among competitors as well as have a positive effect on the company's image and brand. However, if an LSP chooses to remain inactive regarding the adoption of green initiatives this can lead to an increased risk of exposure to criticism and negative press, lagging behind competition as well as losing market shares.

The influence from customers and top management seem to have a prominent role as drivers in the continued development of green initiatives among the studied LSPs. By adopting green initiatives, LSPs strive to improve customer relationships in order to retain existing customers as well as winning new customers. According to the LSPs studied, customers' curiosity regarding green initiatives has increased in recent years, even if there seems to be a certain lack of willingness to invest in and pay for green initiatives. Regarding top management, the findings suggest that a successful adoption of green initiatives and integration of green considerations in the business strategy will not occur without a clear and active support from the company's top management. In addition, the engagement and support from top management can be crucial for how successfully the adoption of green initiatives is integrated into the company and received by their employees, which in turn can be considered as an essential driver and resource when adopting green initiatives. Regarding the barriers studied, financial and economical barriers emerge as crucial when LSPs adopt green initiatives. Uncertain payback period, high investment cost, increased need for external financial support as well as difficulties in justifying investment regarding green initiatives within the company are examples of such barriers. In addition, customers were also mentioned among the studied LSPs as a barrier, for reasons such as lack of customer support, unwillingness to pay for green initiatives, and unclear and unreasonable green requirements from customers.

Not all companies will be exposed to the same type or extent of green pressure; instead, the disparity of perceptions can depend on and be explained by the nature of the company and its characteristics. The findings suggest that size of the company and type of service offerings provided seem to play a meaningful role when adopting green initiatives. When it comes to strategic priority, the findings suggest that smaller LSPs are more keen to meet the customer's immediate requirements than a larger company that prefers to focus on building longer-term strategic market positions. Furthermore, the type of customers also seems to affect LSPs regarding how different drivers and barriers are experienced within the companies. For 
example, the analysis concluded that customers from different industries put varying pressures and requirements on LSPs in regard to green initiatives and solutions.

From a more overall perspective, it does not matter what kind of business change or decision an LSP faces; the response to a new challenge will most likely depend on what kind of business the company is running, and the different requirements the company itself and its customers may have. This implies that type of business, as well as requirements from the LSP itself and its customers, should not be ignored when it comes to adopting green initiatives. Different LSPs may find similar benefits in adopting green initiatives but how these are achieved depends on the LSP's specific firm characteristics in combination with its business context. Therefore, it may require unique solutions adjusted for specific business conditions, as well as customer needs and requirements.

Finally, in addition to how different factors affect LSPs when adopting green initiatives, the findings suggest how green initiatives can be reflected in the service offering. Despite the early phase of LSPs' greening process, the attitude towards a green approach regarding the service offerings seems to differ among the LSPs studied: while some were working towards a green integration in the entire business, others offer green alternatives to the original service offering. The findings suggest possible explanations as to why these differences appear, as well as suggesting different patterns in a green approach in the development of service offerings. Furthermore, the findings suggest that the development of green services includes both the internal work as well as the explicit service offering.

\subsubsection{The connection between the Licentiate thesis and the Dissertation}

The development of green services within the LSP industry can be seen as a fruitful and promising area in responding to increasing green demands of customers as well as to maintain competitiveness on the market. This dissertation continues on from and aims to develop further where the Licentiate thesis ended, namely the development of green service offerings. The findings from the Licentiate thesis provide a foundation in the continuing work to integrate and adopt green initiatives into LSPs and also how this affects their service offerings. The identification and investigation of how different factors affect the adoption of green initiatives among LSPs can be of special interest when it comes to understanding the willingness to develop green service offerings. While the Licentiate thesis has broader approach through its organisational perspective, the focus in the dissertation is narrowed down to concern LSPs' environmental work from a service development perspective, see Figure 1.2.

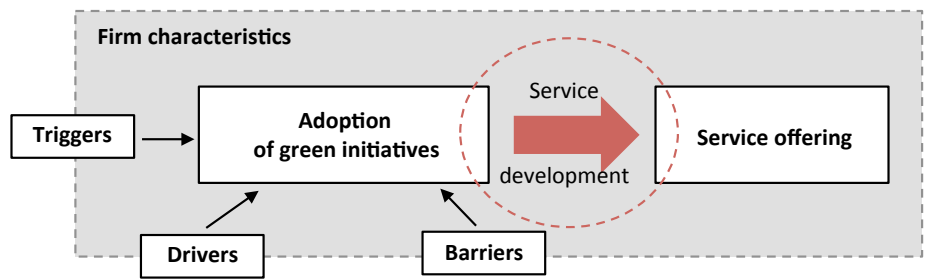

Figure 1.2 The main focus of the dissertation.

However, as indicated above, the development of green service offerings among LSPs is still in an early phase. But the results also reveal an increasing interest regarding service offerings that include green considerations among their customers. This can in turn be seen as a starting point and suggests that green service offerings may be of increasing importance in the future. 
However, this presents LSPs with new challenges. If customers are to be provided with green service offerings, this must to some extent also influence and be integrated into their service development activities and efforts. The extent to which an LSP chooses to address development of green service offerings may depend much on the strategic direction, but also on the company's capability to support the development of green service offerings.

The next section will give a brief introduction and status of the existing research concerning green development among LSPs' business and service offerings.

\subsection{Green development within the LSP industry}

In general, environmental management efforts in the context of the LSP industry have been given only little attention in the literature (Rossi et al., 2013; Lieb \& Lieb, 2010). Despite the lack of research, environmental awareness and adoption of green initiatives has become a topic of great interest among several LSPs. Suggested explanations for this awareness include a corporate desire to do the right thing, pressure from customers, corporate desire to enhance company image, corporate desire to attract green customers and competitive pressure (Lieb \& Lieb, 2010; Isaksson, 2012).

The adoption of green initiatives among LSPs may take various forms. It can be regarded as a way to boost the company's brand in order to strengthen its position on the market and act as an environmentally responsible member of society. According to Lieb and Lieb (2010), brand maintenance issues can become particularly applicable in the LSP industry, where actors offer more or less the same services. Many LSPs have started to include statements regarding environmental awareness in both their business strategies and their operations. Hence, the greening of the business and its brand can be seen as a way for a company to seek a legitimate basis for differentiation of the company's service offerings. The response towards this development can be seen among several LSPs; for example, DB Schenker states that one of their goals is to become a leader in environmentally sustainable transport and logistics solutions (DB Schenker, 2014); DHL states that they view environmental awareness, as well as inclusion of their service offerings, as a way to create value both to their company and also to their customer (DHL, 2014); and PostNord wants to position itself as a sustainable organisation with a focus on environmental concerns that benefits their customers, employees and shareholders, as well as society (PostNord, 2014). In line with Pieters et al. (2012), the reasoning above implies that environmental concerns have become a major source of influence on LSPs' behaviour and strategies as well as improving their competitive advantages. However, the corporate messages often appear to be more of a strategic intent than realised into actions or concrete service offerings. Pieters et al. (2012) identify a knowledge gap regarding how these strategies are translated into real actions and Rossi et al. (2013) stress that capabilities and tools to deploy a strategy for integrating green initiatives within the business seem to be lacking.

Furthermore, another reason for implementing green initiatives concerns the customers. LSPs think it is important, their customers ask them to do so and there is a desire to attract "green" customers. Despite this, LSP customers have been pointed out in the literature as being interested in discussing sustainability issues but do not seem to be willing to allow an economic issues trade-off against environmental sustainability. According to Gammelgaard and Prockl's study (2012), environmental sustainability is regarded as an important issue but the authors argue that environmental sustainability is today expected and viewed as an "add- 
on" factor. This view may, however, change slightly in the future and the authors indicate that manufacturing companies who are customers of LSPs have until now been occupied mainly with their internal environmental work and are just starting to think more about requirements for their logistics and transport services.

Even if customers in general show a reluctance to pay extra for green investments, many LSPs seems to be future-oriented as there seems to exist an anticipation among these actors that the future will bring a change in direction towards a demand for sustainability (Gammelgaard \& Prockl, 2012; Isaksson, 2012). In all probability, a possible future trend is that companies will demand sustainability (Gammelgaard \& Prock1, 2012) and future stringent environmental regulations will speed up this development, which will have implications for the whole supply chain, including the LSPs (Lieb \& Lieb, 2010). This in turn will provide market opportunities for these LSPs that have been future-oriented and developed green knowledge and resources in order to offer their customers assistance and develop new green service offerings. In order to meet set targets and regulations as well as fulfil customers' green demands there will be a greater need to develop new and innovative green solutions. Integrating green concerns into logistics services has been identified as paramount for the LSP industry (Lin \& Ho, 2008). This is seen as a promising research area as well as a practice that has the potential to provide significant benefits to the firm and the society at large (Zailani et al., 2011).

Overall, logistics research literature in regard to knowledge of general service development is scarce, especially in the transportation and logistics industry (e.g. Wagner, 2008; Cui et al., 2009; Busse \& Wallenburg 2011). In addition, Zhou and Wang (2012) state that there is a lack of service development expertise, awareness and skills among LSPs. By adding a green perspective to this research area, the amount of research and number of reports will decrease even more (e.g. Gammelgard \& Prockl, 2012; Zailani et al., 2011; Lin \& Ho 2008). Based on the suggested trend that environmental sustainability is likely to play an increasingly important role in the future, this may place requirements on LSPs regarding how environmental awareness can be reflected and integrated into their service offerings.

However, understanding how LSPs can integrate environmental concerns into their service development efforts raises, in turn, a research need to reveal and investigate different dimensions of service development that LSPs need to take into account in their service development efforts towards the development of green service offerings. Since service development by nature involves a multitude of functions, activities and resources (Menor \& Roth, 2007; 2008), there is justification for taking a broad and holistic approach.

\subsection{Purpose and research questions}

Based on the lack of research into service development, in particular green service development among LSPs, and the broad scope of dimensions that service development embraces, the purpose of the dissertation is the following;

\section{The purpose is to develop and explain a framework for LSP' development of green service offerings}

This dissertation aims to build up an understanding of LSP efforts to develop green service offerings and moreover, for a future orientation, strives to inspire and create a foundation of 
both academic and practical relevance. More specifically, this dissertation aims to give an academic footprint in primarily two research areas, namely green logistics and service development.

First, this dissertation, together with the Licentiate thesis, provides descriptions of the greening status of some LSPs. This description is further developed in an in-depth study investigating both the general environmental work and the integration of green concerns into service development activities and efforts. Research into the area of green logistics includes various research streams that take an environmental perspective on logistics. One such stream that addresses companies and their business conditions is green supply chain management (McKinnon, 2010). However, most writing and empirical studies concerning sustainability in supply chain management have originally focused on manufacturing companies (e.g. Eltayeb \& Zailani, 2009; Hong et al., 2009) and not much attention has been paid to LSPs specifically (e.g. Martinsen, 2014; Lin \& Ho, 2008; Lieb \& Lieb, 2010). Second, research into service development also seems to exist to a limited extent in logistics research in general, in particular in the LSP industry. By integrating green aspects into the service development area, a better understanding can be reached and suggestions can be made for how green logistics and service development can be linked to each other. This, in turn, can be further developed and the results of this dissertation can act as a foundation or a point of departure for future research regarding green logistics as well as service development.

The purpose can thus be viewed as two-part: general, as well as green service development among LSPs, which separately require a deeper understanding in order to be able to answer the purpose. These parts are linked to each other in the sense that an understanding of an LSP's adoption of green initiatives as well as an understanding of how an LSP manages the service development is needed in order to investigate dimensions needed for the development of green services. The next section will give an overview of service development within the LSP industry and also present the research questions for this dissertation.

\subsubsection{Service development within the LSP industry}

Over the past years, the transport and logistics industry has been influenced by several reconstruction and consolidation efforts. In addition, the boundary between the different transportation modes within this sector has largely been erased in order to meet growing customer needs for flexibility, cost effectiveness and international presence. As a result of this change, the range of services offered by LSPs has broadened considerably from transportation and warehousing into more advanced supply-chain solutions (Soinio et al., 2012). Due to increasing competition, as well as more demanding customer requirements, it is argued in the literature that LSPs need to provide more value to their customers than standardised services (Wagner \& Franklin, 2008). For these reasons, a focus on service development can been seen as vital for LSPs to stay competitive on the market. The competitiveness of companies in the LSP industry is increasingly dependent on their ability to add value to customers' bottom lines (Flint et al., 2005). Companies such as LSPs that operate in markets typified by variations in customer characteristics cannot fulfil customer requirements solely by offering standard logistics service offerings. According to Chapman et al. (2003), the logistics industry is a classic example of the birth and development of a vital new service-based industry, transformed from the business concept of transportation to that of serving the entire logistical needs of customers. Due to the fact that different customers have different needs, differentiation is essential for the business of LSPs (Cui et al., 2009) but without jeopardising a balance between customer satisfaction and cost levels for the service offered (van der Veeken \& Rutten, 1998). 
In addressing these challenges and attempting to recognise opportunities that emerge outside traditional business models, there is a need for LSPs to constantly seek new knowledge, "think for the customers", predict and strive to develop new services to meet customers' evolving needs. However, as mentioned earlier, there seems to be a scant amount of research into service development among LSPs, and Flint et al. (2005, p.113) state that: "logistics research has largely ignored the concept of innovation". In this dissertation, the term innovation is interpreted within the frame of service development and the terms are used interchangeably.

According to a study in the German transportation industry, the output of product/service innovations as well as of process innovations is relatively small (Wagner, 2008). This can partly be explained by the fact that the transportation and logistics sector is not amongst the most innovative, because it is a particularly mature industry in which changes are typically evolutionary rather than revolutionary (Mena et al., 2007). But at the same time, Lin and Ho (2008) stress the external environment in which a company conducts its business as a factor influencing the innovative capability as well as intention to adopt innovations. Hence, there is a challenge for the traditional LSP industry to increase its perceptiveness towards changing customer needs.

Furthermore, the modest degree of delivery of new service offerings and innovation can, according to Wagner (2008), depend on insufficiently related strategies, structures, processes and manpower within the LSP's own organisation. This in turn reveals that service development can be regarded as rather complex, as it includes and consists of several different dimensions that need to match and support each other in order to be successful to repeat benefits from the service development efforts. In addition, service development does not solely focus on internal aspects but also needs to address external aspects in order to respond to the market demands. The external focus is highlighted by Chapman et al. (2003), who stress that LSPs need to reappraise the full extent and breadth of their functions, systems and processes, not only within their own business but also in relation to the whole supply chain. These arguments stress the need for a holistic view on how service development efforts can be formally managed within the LSP industry. In line with purpose, the framework needs to include multiple dimensions capturing a wide range of aspects. This reasoning leads to the first research question:

\section{- RQ1: How can LSP' service development be described in the form of different dimensions?}

In order to reach a holistic understanding, the dimensions need to be captured per se as well as how they interact. Each dimension can be captured through a range of pre-requisites, which can benefit a successful service development. To increase the understanding of each of the dimensions and their contributions to service development, the role of the dimensions needs to be explained. In order to tie the framework of dimensions together, the links between the dimensions need to be explained. This means that RQ1 can be further specified into the following underlying questions:

\footnotetext{
- Which are the pre-requisites to address the different dimensions in service development?

- What is the role of the dimensions in service development?

- How are the dimensions in service development linked to each other?
} 
The first research question aims to investigate LSPs' service development from a general perspective. However, in relation to the purpose of this dissertation, the second research question builds on the results of RQ1 and extends the framework by taking a green perspective. This leads to the following research question:

\section{- RQ2: How can various pre-requisites support LSP' green service development?}

Based on the discussion and result from the first research question, the second research question aims to investigate what kind of green pre-requisites are utilised when an LSP develops green service offerings. As the area of green service development is even less researched and certainly less developed in practice compared to service development in general among LSPs, green service development is in consequence addressed to a less detailed extent.

\subsection{Research focus}

The first part of this dissertation investigates service development within an LSP context. This first step is necessary in order to understand the consequences when developing green service offerings. Regarding the term green service offering, this dissertation will apply the same description as mentioned in the Licentiate thesis, where a green service offering is a service offering with an environmental focus, consisting of, or supported by, one or several green initiatives. Green initiatives refer to Martinsen and Huge-Brodin's (2010) description of green initiatives as transport-related measures (e.g. fuels, vehicle technology, mode choice, behavioural aspects, transportation management) and beyond-transport initiatives (e.g. logistics system design, choice of partners, environmental management system, emissions and energy data). In addition, the term environment refers to the natural environment and environmental is in this dissertation interchangeably used with the term green.

This dissertation will have the same perspective as in the Licentiate thesis, namely an LSP perspective. The definition of LSPs and their activities is in line with the definition proposed by Sweeney and Evangelista (2005): "Third-party logistics are activities carried out by a logistics service provider on behalf of a shipper and consisting of at least transportation. In addition, other activities can be integrated into the service offering such as warehousing and inventory management; information related activities, such as tracking and tracing; and value added supply chain activities, such as secondary assembly and installation of products"(p.9).

Since LSPs are in the infancy phase of both their green development and their development of green service offerings as mentioned in the Licentiate thesis, this leads to some limitations in the scope and focus of this research. Furthermore, in line with the argument of Wagner and Busse (2008), due to the limited research of LSPs service development, this dissertation aims to address new service development management for the LSP industry on an aggregated level rather than a subset of LSP actors. However, this dissertation focuses on and aims to reach larger LSP actors, where the strategic focus on service development is of major importance.

The dissertation will provide insights into service development as well as identify essential pre-requisites both from a general and a green perspective when LSPs develop new services. The focus of this dissertation is to create a holistic understanding of LSPs' new service development based on dimensions mentioned in the new service development literature as of 
importance when developing new service offerings. Aspects that are not explicitly investigated and are outside the scope of this dissertation include specific service offerings (other than green service offerings) and LSP features.

\subsection{Areas of contribution}

The research focus of this dissertation regarding service development and its management among LSPs is relevant both from an academic as well as from a practitioner perspective and intends to make a contribution to both these groups. From a practitioner perspective, the result from this dissertation can help and inspire forward-looking LSP managers within business and service development as well as sales and marketing areas to better understand and approach and manage service development within their organisations. This is done both from a general service development perspective and also from a green service development perspective.

From an academic perspective, this dissertation aims to highlight service development within the transportation and logistics industry as an interesting and fruitful area for further research. Furthermore, the results from the dissertation aim to contribute to both the service development literature and the green logistics literature. In addition, the dissertation contributes to general logistics literature.

Moreover, this research aims to create a link between the logistics and service development field, by investigating LSPs' overall service development and its implications when these actors develop green service offerings.

\subsection{Outline of the dissertation}

As mentioned earlier, this dissertation takes its point of departure in the results derived from the Licentiate thesis, which is a compilation based on four different papers. However, this dissertation is written in the form of a monograph. This section aims to give the reader an overview of the dissertation's structure and a brief presentation of the chapters included, see Figure 1.3 below.

\section{Chapter 1}

This dissertation starts with a short background of the research subject and its link to the results found in the Licentiate thesis. This background together with discussions regarding LSPs' service development and green development leads towards the purpose and research questions of this dissertation. Furthermore, this chapter also presents the relevance and focus of this study and clarifies the use of different concepts and terms in the dissertation.

\section{Chapter 2}

The second chapter concerns the methodology approach and strategy of the dissertation and is structured in line with the developed research design. The chapter includes the methodological link to the Licentiate thesis and presentation of how the theoretical and empirical input have been conducted and applied to this dissertation. The adopted analysis strategy as well as reflections on methodological choices is also included in this chapter. 


\section{Chapter 3}

The theoretical input to this dissertation is divided into three different parts, which are represented in Chapters 3-5. The first part, Chapter 3, presents the theoretical perspective, new service development, on which this dissertation builds. The chapter opens with an introduction to the research area, new service development. This is followed by a discussion regarding different dimensions found in the literature to have a significant influence on a company's service development efforts as well as their connection to each other. A conceptual framework of new service development is presented, based on this discussion.

\section{Chapter 4}

This chapter aims to reveal the status of LSPs' service development in prior research as well as reveal different aspects relevant for LSPs to consider in their service development efforts. The structure of the chapter is in line with the identified dimensions in the conceptual framework presented in Chapter 3. Thereafter follows a summarisation of these dimensions as well as their connection to each other in order to identify their overall importance for LSPs' management of service development.

\section{Chapter 5}

The third and final theoretical chapter concerns LSPs' green development and strives to identify factors that may influence and facilitate the development of green services among LSPs. This chapter includes results from the researcher's prior research as well as empirical data derived from the questionnaire survey presented in the Licentiate thesis. However, this empirical data has not been previously presented or published in any research.

\section{Chapter 6}

This chapter includes the empirical input to this dissertation and is divided into three parts. The first part concerns the general service development management at the selected LSP while the second part presents the environmental work as well as its connection to the company's service offerings. The third and last part aims to illustrate an example of a new service development project focusing on environmental considerations.

\section{Chapter 7}

The seventh chapter contains the analysis of this dissertation. The analysis takes its starting point in the developed conceptual framework presented in Chapter 3. The chapter is divided into three parts and follows a step-wise approach. The two first parts aim to analyse and answer RQ1 while the third part discusses RQ2 in this research.

\section{Chapter 8}

This chapter starts with a presentation of the main conclusions from this research. Thereafter follows a discussion regarding the contributions of the dissertation both from a theoretical and practical perspective. In addition, the chapter also includes discussions regarding suggestions and directions for further research within the selected research area.

\section{Appendix}

The appendix attached to this dissertation includes the Licentiate thesis: Logistics service providers going green - insights from the Swedish market. 


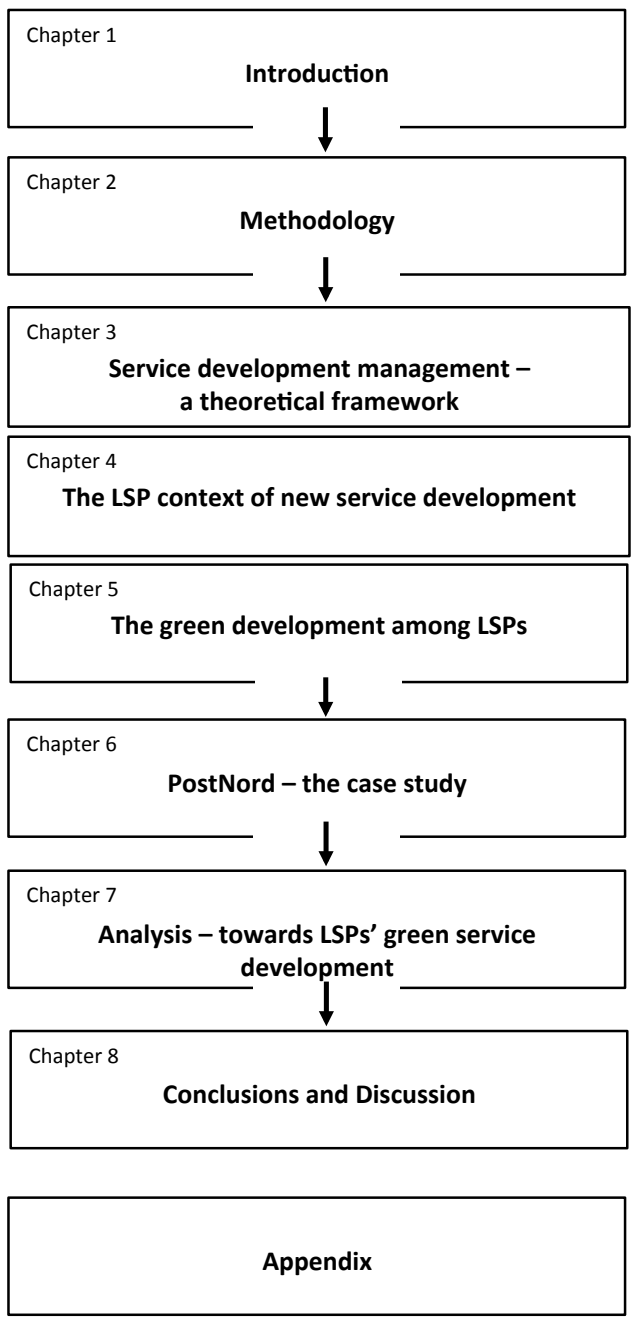

Figure 1.3 The outline of the dissertation. 
- 14 - 


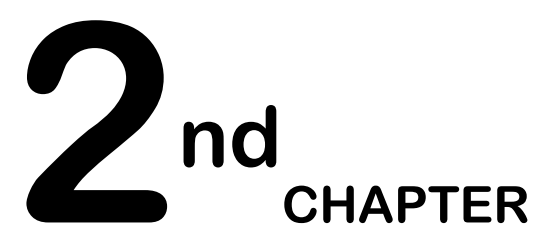

\section{METHODOLOGY}

This chapter aims to reveal the rationale behind the research design of this dissertation. The chapter starts with a presentation of the developed research design and will thereafter guide the reader through its different steps and methodological choices. 
-16 - 


\subsection{The research design of the dissertation}

When doing research, every researcher needs to create a plan, or research design, for how the research will be conducted. A research design can be described as a plan involving procedures of inquiry as well as selection of specific methods of data collection analysis (Creswell, 2009). This is necessary in order to select and form the most appropriate research process for the studied topic. The research design and process developed for this dissertation can be found in Figure 2.1 below, while the research design for the Licentiate thesis can be found in Appendix 1.

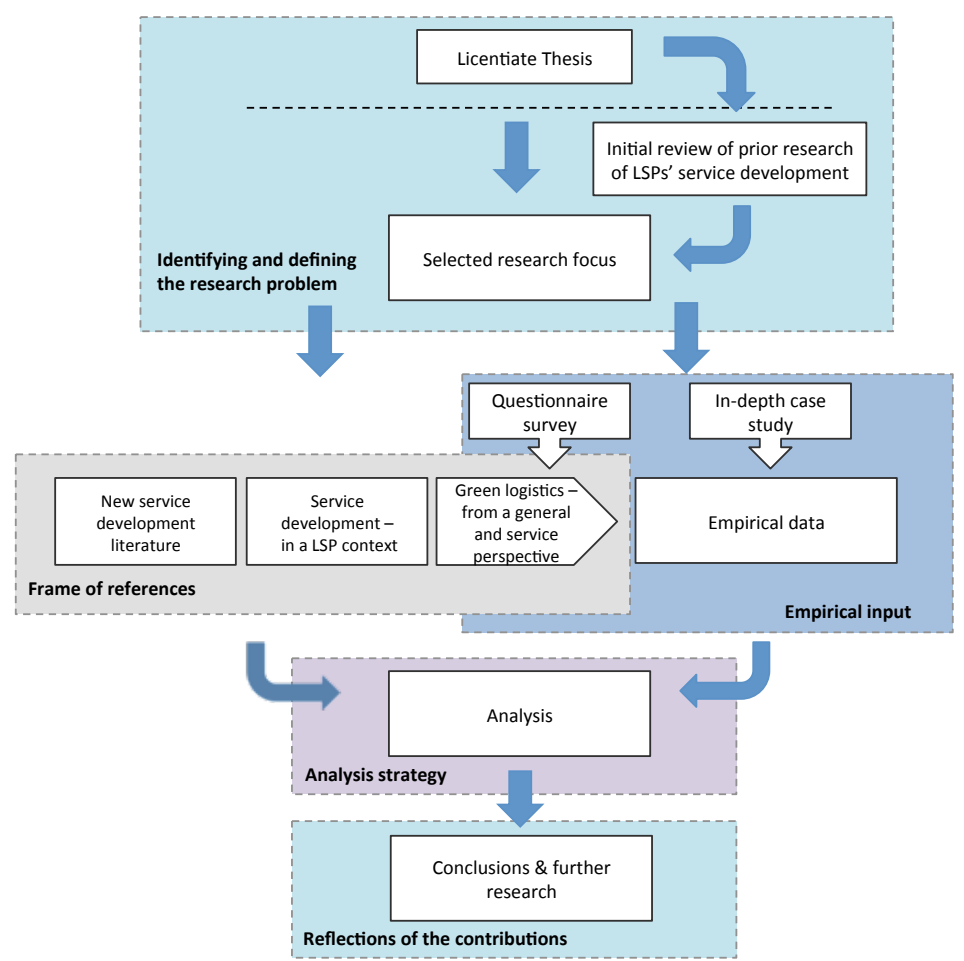

Figure 2.1 The research design of the dissertation.

As can be found in the figure, the research design consists of several main parts that are illustrated by the different colored boxes, which together form the entire research design of this dissertation. Figure 2.1 does not reflect the precise order of the research process; instead it intends to reveal the structure of how it is presented in this dissertation. Each of the main parts will be presented in more depth in the following sections of this methodology chapter. Hence, as a first step, the next section aims to clarify the identification of the research problem as well as the selection of an appropriate research design for this topic.

\subsection{Identifying and defining the research problem}

According to Creswell (2009), the selection of a specific research design depends on the researcher's worldview assumptions together with personal experience, the nature of the 
addressed topic or research problem as well as the audiences for the research study. As illustrated in Figure 2.2, the first part, Identifying and defining the research problem, in the research design aims therefore to describe the identification and definition of the research problem of this dissertation.

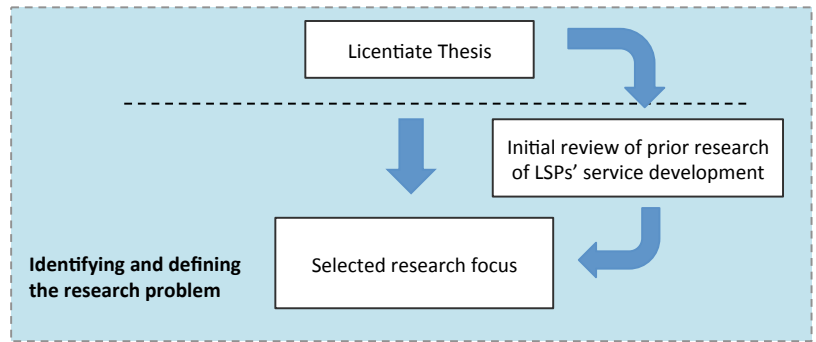

Figure 2.2 The identification and definition of the research problem.

First, in order to give the reader a deeper understanding of the role of the Licentiate thesis and its impact on the dissertation, a further presentation of its research approach, chosen theoretical perspectives and empirical parts is given below.

\subsubsection{The methodological link to the Licentiate thesis}

An overview of the theoretical and methodological/empirical perspective chosen in the Licentiate thesis is presented and showed in Figure 2.3 below.

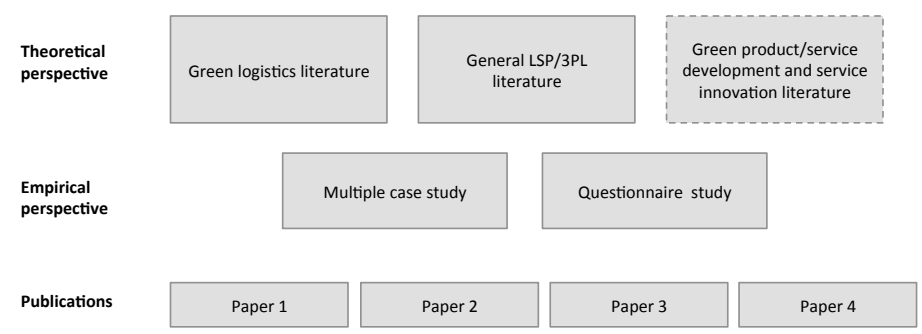

Figure 2.3 The theoretical and empirical contents of the Licentiate thesis.

From the theoretical perspective, different areas of literature have been collected, analysed and used in the different publications as well as in the theoretical framework of the Licentiate thesis. Literature on green logistics and complementing parts of green supply chain management (GSCM) have provided input in the clarification of the "green" status of the LSP industry but also to identify drivers and barriers connected to the adoption of green initiatives. The theoretical framework was also built on a general literature review regarding the LSP industry as well as its service offerings and business development. This review served as a base to gain a deeper insight into this industry in order to be able to relate the findings and the analysis based on the conditions of the LSP industry. These two theoretical perspectives form the main theoretical parts of the Licentiate thesis. Another theoretical perspective used in the thesis, but not to the same extent as the other perspectives, is service literature focusing on product/service development and innovation, both with and without green considerations. This theoretical perspective was intended to serve as a platform to gain a deeper understanding of the underlying mechanism regarding LSPs' approaches towards green initiatives as reflected in their service offerings. 
The empirical inputs have been collected by two different empirical studies, namely a multiple case study and a questionnaire study. The multiple case study represents the major empirical input, while the questionnaire study is seen as a complement to broaden the empirical basis and reinforce or contradict the findings from the case study. In the multiple case study, six different LSPs active on the Swedish market were chosen and investigated. The companies represented two medium-sized LSPs with local/regional business and a wide range of transport and basic logistics services; two small/medium-sized LSPs specialising in express deliveries; and two large international LSPs with a wide range of service offerings. The content of the interviews focused on factors (in the form of drivers and barriers) affecting these companies' awareness and adoption of green initiatives in the company. In addition, there was also a focus on the service offerings and their relation to the adoption of green initiatives in the company. The second empirical study consisted of a web-based questionnaire survey where the targeted sample of respondents consisted of members of two interest organisations, namely Sverges Åkeriföretag and Transportindustriförbund. The questionnaire survey was conducted during 2011 and resulted in 74 responses, which corresponds to a response rate of $12.5 \%$. The following sections of the questionnaire survey were included in the Licentiate thesis: adoption of green transport and supply chain related measures, identification of influencing factors (in the form of drivers and barriers) and also some general characteristics of the companies studied. Since the scope of the questionnaire did not fully correspond to the scope of the Licentiate thesis, not all results from the different sections were analysed. The sections not included concerned areas such as strategic and organisational links to the adoption of green initiatives as well as ICT applications to manage the environmental impact of transport and logistics operations. A further description of the conducted questionnaire survey and its role for this dissertation can be found in section 2.4.2.

The outputs from the theoretical and empirical parts resulted in four different papers, which together with the "kappa" form the full content of the Licentiate thesis. For a more detailed overview of the methodological, empirical and theoretical parts, as well as their connection to each of the papers, see Appendix 1.

The following sections concentrate instead on the research process and the different contents of this dissertation, starting with the researcher's own philosophical views and personal experience of the research problem of this dissertation.

\subsubsection{The researcher's philosophical views and personal experience}

The decision of becoming a $\mathrm{PhD}$ student arose during my time as a student on the MSc Programme, Industrial Engineering and Management at Linköping University, where my economics specialisation was within the areas of logistics management and industrial marketing. My interest regarding both these areas has been a source of inspiration during the research process, whether it was possible to combine these two areas and integrate them within my own research. These years as a PhD student have been full of lessons and experiences, both in terms of increased knowledge of my research area but also of my own research process and philosophical view.

A researcher's philosophical worldviews can be said to lay the foundations for the methodology that will be selected for a specific research problem. In addition, it can also provide insights into why a researcher, from a methodological perspective, chose to design the research in a specific manner (Corbin \& Strauss, 2008). According to Creswell (2009), this worldview is shaped and influenced by the discipline of the researcher together with beliefs of advisers and faculty as well as the researcher's prior research experience. 
Traditionally, logistics research has been dominated by the positivistic paradigm (e.g. Mentzer \& Kahn, 1995; Näslund, 2002) and the positivist-grounded survey approach has been a dominant research method within the logistics area (Mentzer \& Kahn, 1995). However, this trend has changed and logistics research has increasingly been influenced by and based on more qualitative methods, such as case studies, ethnography and action research (Näslund, 2002). According to Mangan et al. (2004), the need for a bridging and a real-world philosophical perspective has emerged with the development of logistics as a field of research. In addition, not all research problems can be solved with the same approach or research strategy. Depending on the research problem, a qualitative approach may be more appropriate than a quantitative approach in some cases, while in other cases a combination of both may be appropriate (Näslund, 2002).

My personal philosophical view holds no full philosophical loyalty to a single paradigm. In that sense, I would perhaps be classified within the pragmatic paradigm. However, instead of trying to "fit in" in a specific philosophical paradigm, I prefer to present important ideals that I strive for during the research process. One ideal concerns having a more holistic view of the research problem in order to carry out good research and answer the purpose and research questions in as a comprehensive way as possible. This is done in order to understand and obtain the entire picture of the research problem and its included parts, as well as the relationships between these parts. These views can be considered to be in line with the system approach, in which Arbnor and Bjerke (1997) define a system as a "set of components and the relations among them". Furthermore, the system approach strives to diminish the importance of people in the studied system and presumes an objective reality that can be "discovered". Another ideal, besides of having a more holistic view and a system approach to the research problem, concerns having an objective viewpoint of the research process and results. Bryman and Bell (2011) describe objectivism as an external viewpoint from which it is possible to view the organisation, which is comprised of consistently real processes and structures.

Like the pragmatic approach, I view the research problem as central and believe that the character of the research problem will influence and shape the research strategy or, in other words, the research design. This includes, for example, decisions regarding which theoretical perspectives and research methods are the most appropriate to adopt for the selected research problem.

\subsubsection{The nature of the research problem}

As mentioned in Chapter 1, the selected research focus of this dissertation emerged from the results of the Licentiate thesis. The selected research focus concerns LSPs' green service development, where green service offerings can be partly seen as an example of a new service concept among LSPs. The result from the Licentiate thesis revealed that the development of green service offerings is at an early stage and is not particularly widespread among the LSPs studied. Despite the early phase, the results also revealed that environmental concerns may become more strategically important in the future in order to maintain competiveness, and also that customers may demand more green service offerings. In addition, through an initial literature review regarding the chosen research topic, the literature helped to substantiate the research problem further. As mentioned in Chapter 1, research regarding LSPs' service development in general is a relatively unexplored research area and is even more so with the integration of a green perspective to this area. Several researchers therefore suggest the relevance of further development, both regarding LSPs' service development efforts in general, as well as development of green service offerings. 
When investigating a topic where the variables and theory base are unknown, a researcher often uses a qualitative approach. Qualitative research is, according to Creswell (2008), explorative in its nature and is adopted when the researcher wants to describe a research problem that can best be understood by investigating a concept or phenomenon. In addition, this approach is appropriate when the research topic is new and has never been addressed within a certain sample (Eisenhardt, 1989), and also when existing theories do not apply to the particular group (Creswell, 2008, p.18).

Due to the immature state of the selected research problem of this dissertation, the strategy of inquiry is therefore a qualitative approach towards further investigating this area. The new service development phenomenon has been studied before within other service companies' contexts but is not particularly developed within the setting of LSPs. Thus, the theoretical base of this dissertation relies on NSD (new service development) literature from an operation management perspective in order to identify and understand the dimensions and pre-requisites when developing service offerings. This is done in order to better understand the implications and how this can affect LSPs that choose to develop green service offerings to their customers.

Regarding the relationship between theory and research, the research process of this dissertation can be characterised in accordance with Bryman and Bell (2011) as having a more deductive approach. The research process takes a clear starting point in the NSD literature, which in turn acts as a way to guide and drive the process of gathering data.

\subsubsection{Defining purpose and research questions}

Choosing and defining a research problem, especially in qualitative research, is often seen as difficult (Corbin \& Strauss, 2008) but is at the same time the most important part of the entire research process (Creswell, 2009). The statement or purpose of the research problem sets out the objectives and reveals the intent of the research problem in the form of what the researcher wants to do and why (Creswell, 2009).

The purpose of this dissertation is to:

\section{develop and explain a framework for LSP' development of green service offerings}

This research aims to explain and increase the understanding of LSPs' development of green service offerings. The study has an organisational inside-out perspective, focusing on relevant service development dimensions and pre-requisites relevant for LSPs to consider when developing green service offerings.

The need for, and identification of, a specific research problem leads to the definition of the purpose, which in turn is refined into specific research questions. The research questions can be seen as a guide as to how the study will be further designed, and according to Corbin (2008), the formulation of the research questions is important since it determines the research methods which will be adopted for the specific study. Bryman and Bell (2011) stress the importance of research questions since they will help the researcher to:

- guide the literature search;

- guide the decisions about the kind of research design to employ;

- guide the decisions about what data to collect and from whom;

- guide the analysis of the data; 
- guide the researcher writing up the data; and

- $\quad$ stop the research from going off in unnecessary directions (p. 83).

The authors also stress that the research questions should be clearly formulated, neither too broad nor too narrow, researchable and that there should be a certain link or consistency between the different research questions. In addition, the research questions should be connected with established theory and research and they should also have a potential for making a contribution to some extent to the research topic.

The first research question in this dissertation is:

- RQ1: How can LSPs' service development be described in the form of different dimensions?

a. Which are the pre-requisites to address the different dimensions in service development?

$b$. What is the role of the dimensions in service development?

c. How are the dimensions in service development linked to each other?

In order to make the first research question more specific and limited, some research subquestions have been developed. The first research question is seen as a first step to answering the purpose and is needed in order to lay a foundation for the following research question. Based on the results from the first research question, the second research question aims to investigate the implications and effects of the result of LSPs developing green service offerings.

The second research question of this dissertation is:

\section{- RQ2: How can various pre-requisites support LSP' green service development?}

The second research question links back to the purpose, and the contribution of this question is both the identification of pre-requisites needed when developing green service offerings and also suggested explanations of why they are needed.

The research questions' link to the reviewed literature in this dissertation, together with the role of the literature and the extent to which it is used for the different research questions, is further described in section 2.3.

\subsection{Theoretical perspective of this dissertation}

The different literature areas that the theoretical understanding builds upon in this dissertation can be found in Figure 2.4 below. This section aims to describe the aim, content and link between the different literature parts together with its search methods. In addition, the theoretical link to the different research questions will also be presented. 


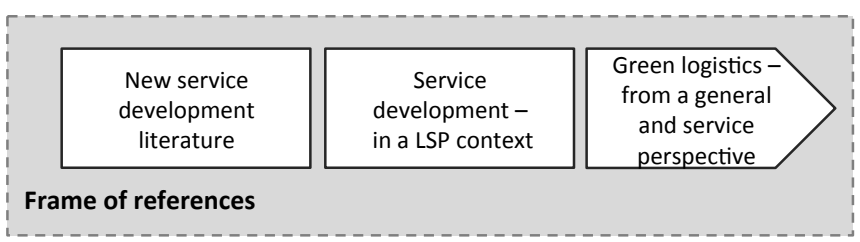

Figure 2.4 The theoretical perspectives of the dissertation

\subsubsection{The aim, content and link between the different literature parts}

First the new service development literature, which can be found in its entirety in Chapter 3 . This literature area is used in order to create a base for the understanding of new service development (NSD) and also to identify key NSD dimensions and pre-requisites needed for a company's NSD activities and efforts. Due to the lack of NSD studies concerning service companies, it also includes studies on NSD and innovation management regarding manufacturing companies. As a first step for identifying pre-requisites when a company develops new services, a number of key NSD dimensions reflecting a company's NSD efforts were selected.

Chapter 3 is structured around these dimensions and each of these dimensions is further explained, modified and complemented with other relevant aspects found in the literature. A summary of the specific dimensions can be found at the end of the presentation of each dimension. This summary is used as a tool in the analysis effort and aims to support and facilitate the comparison between general service development literature and literature regarding LSPs' service development. Since research has paid little attention to NSD in a logistics context, studies on NSD and innovation management in service companies as a whole can provide useful insights.

A tentative framework structure for management of NSD is developed at the end of Chapter 3. According to Voss et al. (2002), building and developing a conceptual framework can act as a way to carefully consider and select relevant constructs and variables included in the specific study. The tentative framework in Chapter 3 includes the different dimensions identified in the literature and reveals their role as well as their link to each other. The tentative framework can be seen as this dissertation's analysis model and is therefore used to guide the content of the analysis. In addition, the model is also used to structure the content of the literature of LSPs' service development.

The first literature area was necessary in order to set the stage and limitations for the content of the second literature area: service development in a LSP context, which also includes literature from the innovation research field. The content of this area can be found in its entirety in Chapter 4. This literature concept aims to reveal the status of service development among LSPs by investigating each of the dimensions mentioned above in the setting of LSPs. Due to the lack of literature regarding LSPs' service development, relevant literature regarding outsourcing, third-party logistics, value added services as well as organisational learning among LSPs has been used as a complement and interpreted from a service development perspective.

Chapter 4 ends with a concluding discussion, linking the different dimensions together and discussing their relevance from an LSP perspective. Some survey questions are developed based on this discussion. These questions do not have an analytical purpose; rather, they are descriptive and work as a base for the empirical study. 
The second literature section is used to create a base and increase the understanding of the general service development among LSPs in order to partly identify which opportunities and challenges LSPs may face when developing green service offerings. The third literature area, green logistics - from a general and service perspective, can therefore be seen as an extension of the second literature area. The complete compilation of this area can be found in Chapter 5. Due to the scarcity of green service development studies among LSPs, this literature area has been extended to include literature regarding general green logistics in the context of LSP. In addition, results from the Licentiate thesis have been included in this chapter. This includes results concerning ways in which the adoption of green initiatives can be reflected in LSPs service offerings, and also results regarding implications of different drivers and barriers towards an LSP's adoption of green initiatives within their own company.

Based on the discussion above, the different literature key concepts therefore follow a sequential order, where Chapter 3, as a first step, acts as a base and determines the framework for what to investigate. In the second step, Chapter 4, the results of Chapter 3 are applied in an LSP context. The third and final step, Chapter 5, acts as an extension of Chapter 4, illustrating green logistics services as an example of the development of a new service offering.

\subsubsection{Search methods for the different literature areas}

As mentioned above, this dissertation builds upon three different literature areas. The literature search approach for each has differed slightly depending on the extent of the respective research. As a consequence, articles and literature connected to these areas have been identified by using different sources and search approaches. As a first step, a literature review of the first literature area, new service development literature was conducted. This review was based on a keyword search conducted in the research database Business Source Premier. The search terms used were service development combined with terms such as framework, model, strategy, concept and process. These key words had to be included in the abstract of the reviewed articles and the search result was also limited to peer-reviewed academic articles. The intention with the literature review was to find relevant dimensions and pre-requisites for new service development.

Literature and articles for the second literature area, service development in a LSP context, were also found by adopting a keyword approach. However, the search was conducted through the web search engine Google scholar. This was done in order to increase the rate of relevant hits and focused not only on academic articles but also on books and reports within this area. The search terms were in line with the keywords mentioned above but were also extended with synonymous terms such as service management and innovation in combination with $L S P, 3 P L$ and logistics. In addition, a snowball approach was adopted, based on the references in the articles found, in order to find more relevant reports and literature regarding LSPs' service development management.

Regarding the third literature area, green logistics from a general and service perspective, a major part of the included literature was derived from the researcher's prior research (for example, research findings from the Licentiate Thesis). However, a complementary search on search engine Google scholar and on the web was conducted in order to find additional articles, studies, reports and books. The keywords for this search were similar to the terms mentioned above. These terms were used in different combinations together with a third search term criterion: green, environment and sustainable. Moreover, a snowball approach was also used within this area in order to find additional literature. 


\subsubsection{The theoretical link to the research questions}

The theoretical input and link to the research questions of this dissertation is illustrated in Figure 2.5 below.

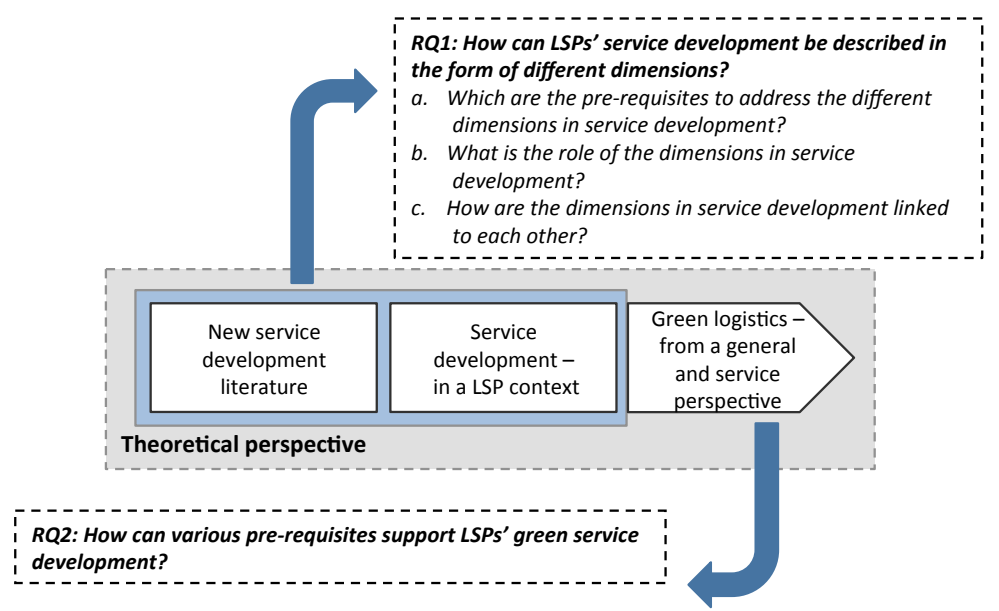

Figure 2.5 The theoretical link to the research questions.

The first and second literature areas are primarily linked to RQ1 and to its underlying subquestions. The theoretical input from the first literature area aims to support RQ1 by suggesting different service development dimensions and pre-requisites, their role and relevance as well as connection to each other from a general service development perspective. While theoretical input from the second literature area will act as help to modify, complement and specify these dimensions and pre-requisites from an LSP perspective.

While RQ2 builds on the results of RQ1, the theoretical input from the third literature area strives to give a deeper understanding of RQ2. It should give inspiration, and reveal prerequisites relevant to consider, as well as explanations that may influence the development of green service offerings among LSPs.

\subsection{Empirical input to the dissertation}

The different empirical input to this dissertation is illustrated in Figure 2.6 below. Further, this section aims to present and discuss the preparation of these empirical studies as well as their role and purpose for this dissertation.

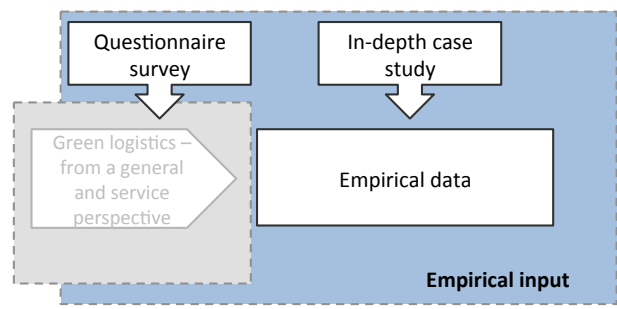

Figure 2.6 The empirical inputs to the dissertation. 


\subsubsection{The case study}

In general, a case study approach is often selected when theory may be lacking or if the environmental context is different (Stuart et al., 2002). The case research methodology is often adopted in exploratory studies where the selected phenomena and its underlying variables are neither well-addressed nor understood (Meredith, 1998). In addition, case study research allows and provides a more holistic view on the studied topic, e.g. organisational or managerial processes (Yin, 2009). For these reasons, a case study approach was choosen based on the topic in this dissertation.

Whether to choose to conduct a single case study or a multiple case study is largely based on the purpose of the research. Single case studies are often chosen when the researcher strives for a rich description of the existence of a phenomenon (Siggelkow, 2007). For that reason, an in-depth single case study is selected in this dissertation in order to describe the service development management and development of green service offerings for a specific LSP.

The selection of the specific company in this dissertation was based on the following criteria:

- Be an actor in the LSP industry on the Swedish market

- Be of sufficient size to have resources available to engage in service development efforts

- Have one or more on-going service development project of strategic importance

- Show demonstrable ambition regarding green initiatives connected to its operations

The selected case company in this dissertation fulfils all the criteria mentioned above and is also an example of an LSP that has announced their intention to grow in the near future, which in turn can have implications for improving the ability to be more service-oriented.

The aim of the case description in this dissertation is to provide illustrative examples, contribute to concretising the developed framework and create an understanding of the prerequisites of LSP's service development. The case description also contributes to the understanding of the environmental work and how this can affect the development of green service offerings among LSPs.

The case description, which can be found in Chapter 6, is divided into three parts. The first part, Introduction and Service Development Management, gives an overview of the selected LSP and continues thereafter with a more comprehensive description of the company's service development management and efforts. Regarding the link to the research questions and the analysis, the first part of the case description mainly gives empirical input to RQ1. The second part, Environmental work and Green service offerings, concerns information on the company's general environmental work, the existence of green service offerings as well as considerations of the development of green service offerings. The third and final part, City logistics - an example of a new and green service concept, aims to illustrate a new and strategic service concept within the selected LSP, where integration of environmental considerations has an essential role. The second and third parts of the case study aim to give empirical input to RQ2.

In this dissertation, the in-depth case study is based on qualitative data, which has been collected primarily through interviews. The conducted interviews have had a more semistructured character, which is recommended for explorative studies (Yin, 2009). 
Before each interview, the respondents were informed of the purpose of the interview and were given an outline of the content of the interview questions. This was done in order to inform the respondents of the researcher's intention with the interview but also to give the respondent the opportunity to prepare for the interview. All the interviews, apart from one (a telephone interview) were conducted at the case company's site. In total, five different respondents were interviewed and the interviews lasted from one to three hours. For each interview, an interview guide was developed, in which the content and questions were inspired and determined by the initial literature review. Some parts of the interview guides overlapped; however, this was in order to obtain a richer picture as well as to identify differences in the answers from the respondents. This can also be seen as a way to triangulate the collected data and thus strengthen the construct validity of the case study (Voss et al., 2002). The titles of the selected respondents and their contributions to the different parts of the case report are shown in Table 1 below.

Table 1 The repondents contributions to the case descrpition.

\begin{tabular}{|c|c|c|c|}
\hline The respondents & Part I & Part II & Part III \\
\hline Vice president, Business Development, PN Logistics, PostNord & $\mathbf{x}$ & $\mathbf{x}$ & \\
\hline Product Specialist, Market \& Sales, PN Logistics, PostNord & $\mathbf{x}$ & $\mathbf{x}$ & $\mathbf{x}$ \\
\hline Environmental Manager, PostNord & & $\mathbf{x}$ & \\
\hline Environmental Manager, PN Logistics, PostNord & & $\mathbf{x}$ & $\mathbf{x}$ \\
\hline Senior Project Manager, PostNord & $\mathbf{x}$ & & $\mathbf{x}$ \\
\hline
\end{tabular}

Each of the interviews was recorded, and shortly thereafter the interview was transcribed and compiled into a draft of the respondent's answers. Thereafter, each of the respondents was given the opportunity to review a draft from the interview. Two of the respondents corrected and gave comments on the draft. This was done in order to strengthen the construct validity of the case study. The draft interviews were then combined and grouped into categories inspired by the identified dimensions derived in the tentative framework in Chapter 3. Good documentation of the collected data, in combination with attention to the processing and coding phase of the data, gives a positive result of a study's reliability (Voss et al., 2002). In addition, the complete description of the case study was sent to the company in order to give the respondents the opportunity for a final verification of the content in the case report, which can be seen as a way to further strengthen the construct validity of the study.

The case description has also been complemented by different types of secondary data. For example, additional information on the company has been collected from the company's homepage, annual reports, company reports as well as internal presentation material. This was done in order to triangulate the collected data. Triangulation of data across different sources is also seen to strengthen the construct validity of the case study (Stuart et al., 2002).

\subsubsection{The questionnaire survey study}

Even if the case study comprises the main empirical input to this dissertation, it has been enriched by empirical input from the questionnaire study that was conducted for the Licentiate thesis. The questionnaire survey was a joint research initiative with the University of Naples Federico II (Italy) and also involved other academic research institutes, namely Dublin Institute of Technology (Ireland), Tampere University of Technology (Finland) and 
Herriot-Watt University (United Kingdom). The questionnaire survey aimed to investigate LSPs' adoption of green initiatives and resources as well as drivers and barriers for these initiatives. The Swedish version of the questionnaire survey was conducted during the period June to September 2011. The questionnaire had 590 relevant respondents, who were members of the interest organisations Sveriges Åkeriföretag and Sveriges Transportindustriförbund. By the end of the response period, 74 responses had been received, which gave a response rate of $12,5 \%$. For a more detailed presentation of the questionnaire survey see Appendix 1.

The empirical data derived from the questionnaire survey to this dissertation has not been presented earlier and can thus be considered as "new". The chosen empirical parts concern data regarding different transport and supply chain-related measures adopted by LSPs to improve the environmental impact, different functions in LSPs involved in managing green initiatives as well as the relevance and adoption of ICT applications among LSPs when managing environmental impact. The complete version and full scope of the questionnaire can be found in Appendix 1.

The analysis of the selected questions was carried out by investigating the means of these questions. In addition, t-tests on a level of $95 \%$ were used in order to reveal significant differences between the means. The results of the analysis of these questions are presented in Chapter 5. The role of this empirical data is to enrich the literature in Chapter 5 and act as explanation factors in the analysis of pre-requisites needed for developing green service offerings.

\subsection{Analysis strategy and unit of analysis}

This section aims to present the analysis strategy of this dissertation as well as present the unit of analysis and analysis model, see Figure 2.7 below.

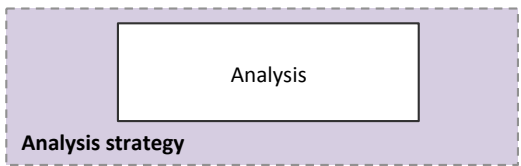

Figure 2.7 The analysis strategy of the dissertation.

\subsubsection{Unit of analysis and analysis model}

One important step that influences and facilitates the entire research design and process as well as the completion of the analysis is choosing the appropriate unit of analysis. The unit and level of analysis can be seen as a way to isolate and demarcate a specific problem area (Stentoft \& Halldorsson, 2002), but also as a way to structure an empirical research study and understand its findings (Menor et al., 2002).

In this dissertation, the unit of analysis can be seen as twofold. For RQ1, the unit of analysis focuses on LSPs' service development from a general perspective, while the unit of analysis for RQ2 concerns development of green service offerings. Due to the nature of the selected research problem, the unit of analysis is viewed from a strategic and rather high level in the company. Thus, the level of analysis in this dissertation is conducted from a company level. In order to further specify the unit of analysis and clarify the variables investigated and analysed, the tentative framework of NSD developed in Chapter 3 is used as an analysis model. 


\subsubsection{The analysis strategy for the case and its link to the RQs}

The overall structure of the analysis, the analysis model as well as the link to the different research questions is illustrated in Figure 2.8 below.

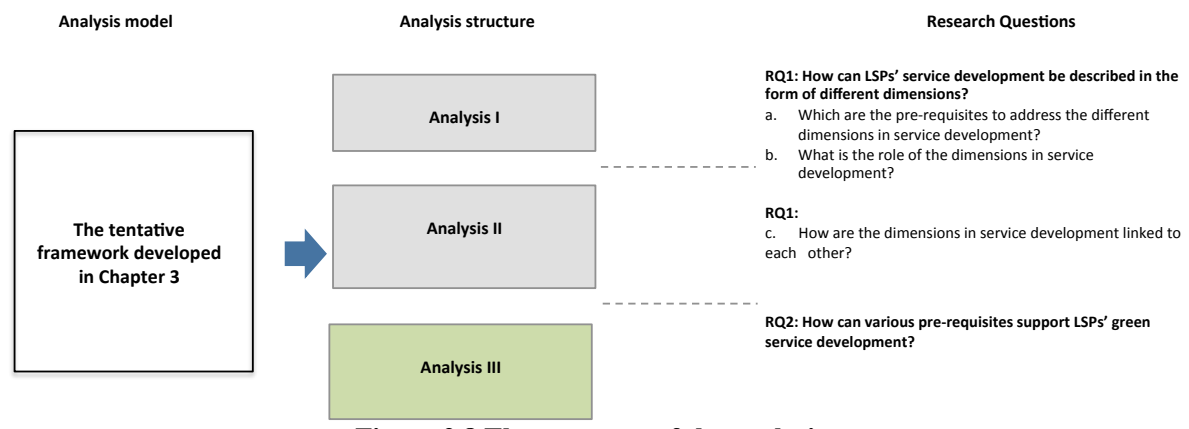

Figure 2.8 The structure of the analysis.

The structure of the analysis follows a stepwise approach and is divided into three main parts. The first part concerns all of the identified dimensions in the analysis model and each of these is analysed systematically and separately. The analysis of each dimension compares the literature findings from the new service development framework (Chapter 3) with the literature findings of each dimension from an LSP perspective (Chapter 4). This was done in order to identify similarities and differences between these areas. The findings from the new service development framework acted as an inspiration source for what an LSP might consider in the development of new service offerings and how. In addition, empirical input from Chapter 6 also provided illustrative examples, descriptions and understanding of how an LSP acts and considers each of the different dimensions. The output of the first part of the analysis was to define the role of each of these dimensions as well as break these down into pre-requisites relevant for LSPs to consider at the management of service development. Regarding the link to the research questions, the first part of the analysis concerns first and foremost RQ1a-b and thus provides a base in order to be able to answer these questions.

The second part of the analysis aims to link these dimensions together and analyse the impact and relevance of the dimensions both to each other and to the identified pre-requisites. Based on the findings from the first part of the analysis, the output of the second part resulted in a further developed framework of the analysis model. The developed framework is adapted and modified from an LSP perspective and reveals dimensions and pre-requisites needed for service development. The second part of the analysis aims to answer RQ1c.

The third part of the analysis aims to analyse the framework developed in the second part of the analysis from a green perspective. The literature findings and discussions from Chapter 5 acted as an input to this analysis. This was done in order to analyse how the identified prerequisites might be modified or complemented when an LSP chooses to develop green service offerings. The analysis in this section is more empirical driven, based on input from the case study (Chapter 6), regarding the studied LSP's environmental work and development of green service offerings.

In addition, results from the multiple case study presented in the Licentiate thesis was also used to support and strengthen the analysis in order to emphasise the relevance of prerequisites regarding development of green service offerings (for a more detailed 
methodological description of the conducted multiple case study, see Appendix 1). This part of the analysis aims first and foremost to provide answers to RQ2.

\subsection{Reflections upon contributions of the dissertation}

In general, borrowing perspectives and theories from other disciplines has been recommended in order to enrich logistics research (Stock, 1997), contributions which can help to increase the understanding of different strategies and processes within the logistics research field (Nilsson \& Gammelgaard, 2012). In this dissertation, the theoretical input takes a clear starting point in the service discipline by having a strong focus on the phenomenon of NSD and service development management. By linking and comparing this literature with characteristics and constraints of LSPs' service development efforts, this study seeks to synthesise key NSD dimensions and form a framework for LSPs concerning development of green service offerings.

The overall ambition and core of this dissertation is to make a contribution to the body of logistics literature. The ambition is not to develop and generate new theory; rather, to expand and enrich the available literature and research regarding LSPs today. In addition, the wish is to highlight and create understanding and knowledge of the service development area within LSP research, which has previously received little focus. However, this is an area that will, in all probability, affect LSPs to a greater extent in the future, given the fact of the market's increasing and changing requirements. This applies both to an LSP's service development in general but in particular to meeting the market's demands with regard to the development of green and sustainable service offerings and solutions. Due to the strong focus on NSD and service development management, the research also aims to make a contribution to the general service development literature, where LSPs can be seen as an example of a service company.

The overall process of this dissertation concerns the creation of a general framework for LSPs' service development in order to be able to investigate how LSPs can improve their ability to develop green service offerings. This means that the output of this research will result in increased knowledge about both general service development among LSPs and also the necessary pre-requisites for the development of green service offerings. 


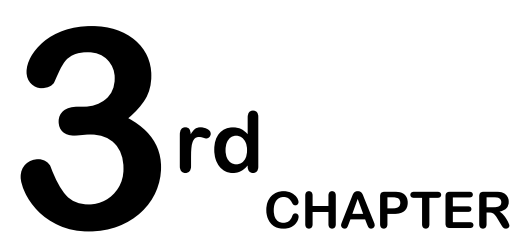

\section{SERVICE DEVELOPMENT MANAGEMENT - A THEORETICAL FRAMEWORK}

This chapter includes the theoretical perspective on which this dissertation builds. The theoretical foundation of this dissertation is phenomenon driven rather than theory driven since it aims to describe the phenomenon of new service development and the influencing factors needed in order to succeed with the development of new services. The chapter starts with an introduction to the new service development research area and continues thereafter with a discussion of different dimensions found in the literature to have a significant influence on new service development. Thereafter follows a section describing the role of each dimension as well as its connection to each of the others in the management of service development. A conceptual framework of new service development is presented, based on this discussion. 
-32 - 


\subsection{Introduction to service development}

New service development (NSD) has emerged as both an important research topic and a critical aspect of business strategy in both service and non-service industries (Menor et al., 2002). As firms have become more service-oriented they have recognised the need to compete on the basis of new service offerings. This is largely due to the changing business environment many service firms face in the form of increased globalisation, growing competition, introduction of new technologies and changing consumer demands (Ottenbacher \& Harrington, 2010). This new business environment has forced these firms to respond constantly to these changing conditions and to focus on service development, placing it at the core of their competitive strategy. To maintain market share and stay ahead in the modern marketplace, business firms must constantly look for innovative strategies that will improve their competiveness (Chapman et al., 2003) in order to find new ways to create and increase value to the customers (Pekkarinen \& Ulkuniemi, 2008). It has been suggested in prior research that competing in a rapidly changing market often requires the capability to develop quickly and provide new offerings (e.g. de Brentani, 1991; Johne \& Storey, 1998; Kelly \& Storey, 2000).

NSD can be described as a multi-dimensional construct that reflects both operational effectiveness and marketplace competiveness (Menor et al., 2002) and can thus be considered as a crucial means to achieving and sustaining superior competitive advantage (Ordanini \& Maglio, 2009; Lusch et al., 2007). Furthermore, the development of new services requires a unique combination of different resources, as well as personal skills on the part of both the producing service firm and customers (Vargo \& Lusch, 2004). The success of the services will depend on how these resources and skills are configured and put together (Lusch et al., 2007).

However, organising NSD is not an easy task and many firms face the challenge of how to best manage their development of new services (Menor \& Roth, 2008). Those firms that succeed can expect to see benefits such as enhanced profitability of existing offerings; new customers being attracted to the firm; greater loyalty of existing customers; and markets of opportunities opening up (Storey \& Easingwood, 1999).

\subsubsection{Types of new services}

When investigating NSD, it is important to take into account the fact that not all services are the same. A service can vary considerably in terms of the nature of the service act and of the degree of interaction between the service organisation and the customers (Storey \& Hull, 2010). For example, de Jong and Vermeulen (2003) mention two different kinds of services, namely production-intensive services and consumer-oriented services. The productionintensive service are characterised by simplification but also by standardisation to particular user needs. Examples of production-intensive services include transport, banks, insurance and wholesale services. Consumer-oriented services, on the other hand, have a continuous incremental nature due to high knowledge-intensity, such as in scientific, engineering and IT services. As a result of the diversity of service offering, in combination with poor understanding of it, it has proved difficult to identify general principles for managing operation and marketing practices across different service firms (Chase \& Apte, 2007).

Several definitions or classifications of new services have been offered in the literature (Menor et al., 2002). Shostack (1987) describes services as a series of interactions between 
participants, processes and physical elements and maintains that any change to the service concept that requires competencies which differ from the existing operation can be considered as a new service. The distinction of what constitutes a new service is, according to Menor et al. (2002), significant both when it comes to determining the appropriate mix of services in the product portfolio offered and in terms of understanding how the customer or the marketplace perceives the new services. New service development can therefore be classified as ranging from true innovations, which are totally new-to-the world services with an entirely new market, through to fairly minor modifications of existing company services (Ottenbacher \& Harrington, 2010).

A classification commonly used in the literature for new service development or service innovation is the classification by Booz Allen Hamilton, which was originally developed for classification of new products (Johne \& Storey, 1998). The classification shows the range of different kinds of services, which is used as the base later on in this dissertation when discussing whether different kinds of services and levels of innovativeness may require different types of resources, skills and knowledge when developing these services. The classification includes the following levels of innovativeness in NSD:

- New-to-the-world services: new services that are seen to be completely new in the eyes of the customers because they are the first of their kind, creating entirely new markets.

- New service lines: services that are not yet new to the marketplace but are new to the firm.

- Additions to an existing service line: new services that supplement a company's established service line and are not significantly new to the service producer, but may be new to the customers in the existing market segment.

- Improvements and revisions to an existing service: new services that provide improved performance or greater perceived value and so replace existing services.

- Repositioning: existing services that are targeted at new markets or market segments, or a new use of a new competitive set.

- Cost reductions: new services that provide similar performance at a lower cost of supply. (p. 189)

The previous subsections have defined NSD and the role it plays for service firms, as well as placing attention on the span of innovativeness when developing new services. The following subsections will examine the strategic relevance of NSD and discuss factors needed in order to reach a strategic state of NSD within a firm.

\subsubsection{Why a strategic perspective on NSD?}

The literature on NSD can be described as consisting of three different research streams, namely the service research stream, the product development stream and the innovation stream (Edvardsson et al., 2013). Even though NSD is not a new research area, it has been viewed as the least studied and understood topic in the service management literature (Menor et al., 2002). In the study by Papastathopoulou and Hultink (2012), the authors examine the state of the art in NSD research during 1982 and 2008. The authors found that although NSD research dates back almost three decades, half of the articles have been published in the last 10 years. 
Despite its criticality, many authors have claimed that empirical insights into new service development have not been developed or advanced (e.g. Froehle \& Roth, 2007; Menor \& Roth, 2007; Menor et al., 2002) and have called for more conceptual and empirical NSD research (Page \& Schirr, 2008).

Management of service development has been identified as an important competitive concern both in research and in many service industries for some time. However, the current understanding of the strategies, methods, critical resources and activities used to develop new services seems still to be inadequate given the importance NSD has as a competitive driver (e.g. Edvardsson et al., 2013; Menor et al., 2002). In addition, Froehle et al. (2000) state that if NSD is to make a significant contribution to corporate performance, strategic factors for NSD speed and effectiveness must be more rigorously understood and better implemented. There is, therefore, a need for research regarding key strategic factors that influence NSD performance (Edvardsson et al., 2013).

\subsection{Towards a framework for NSD}

Having a strategic focus regarding new service development and innovation puts constant pressure on a service firm to develop more effective NSD methods and make better utilisation of the existing resources as well as increasing competitive awareness in order to offer more attractive service offerings to customers. Even though strategic influences and key factors have been identified as important when developing new services, there seems to be a lack in NSD literature and research linking business strategy and service development (Goldstein et al., 2002).

Successfully developing new service offerings is not a matter of effectively managing only one or two activities connected to NSD. Rather, it has been shown to be a result of a more comprehensive approach that manages to coordinate a large number of aspects competently and in a balanced manner (Johne \& Storey, 1998).

The dimensions mentioned in the literature and prior research which are likely to influence the performance of new services can be based on certain choices regarding organisational, technological and process design (Froehle et al., 2000). Furthermore, choices made regarding these dimensions have been shown to have strategic influence on the firm's ability to develop new services rapidly and effectively. Examples of different studies that take a broader perspective of NSD and present some kind of framework for what makes NSD successful include work done by, for example, Ordanini and Maglio (2009), which focuses on market orientation, internal process organisation and external network; Froehle and Roth (2007), which takes a process and resource perspective, and develops a framework for organising various organisational practices and activities related to NSD; Edvardsson et al. (2013), which investigated the role of service development strategy, a formalised development process, integrated development teams and customer co-creation; and Menor and Roth $(2007,2008)$, which integrates a process and resource perspective with managerial perspectives of NSD by organising various practice constructs for NSD activities.

In their study (2007), Menor and Roth argue that success in NSD comes from building skills in the management of service development resources and organisational routines. The authors conceptualise five different dimensions that jointly reflect a firm's NSD competences. The 
different dimensions and a short description of the content for each of these dimensions are described below:

- NSD process focus: the existence of a formalised process which facilitates and supports the managing of NSD efforts.

- Market acuity: the firm's ability to collect information about market environment and the ability to use this information in a manner that supports the NSD.

- NSD strategy: an enabler for management to plan for and commit the resources required for specific NSD projects.

- IT experience: the use of IT as a facilitator for internal and external coordination of activities and information flows in the development of new services.

- NSD culture: this represents the values and attitudes demonstrated by the service firms that indicate a willingness and desire for new service development activities.

Each of these dimensions has been identified in previous NSD and innovation literature as being organisational resource or routine which is essential in order to have an impact on NSD performance. Menor and Roth (2007) present a conceptual model of NSD competence dimensions in which the authors show that NSD performance is influenced by a multidimensional NSD competence dimension, which in turn is represented by a second order factor, see Figure 3.1 below.

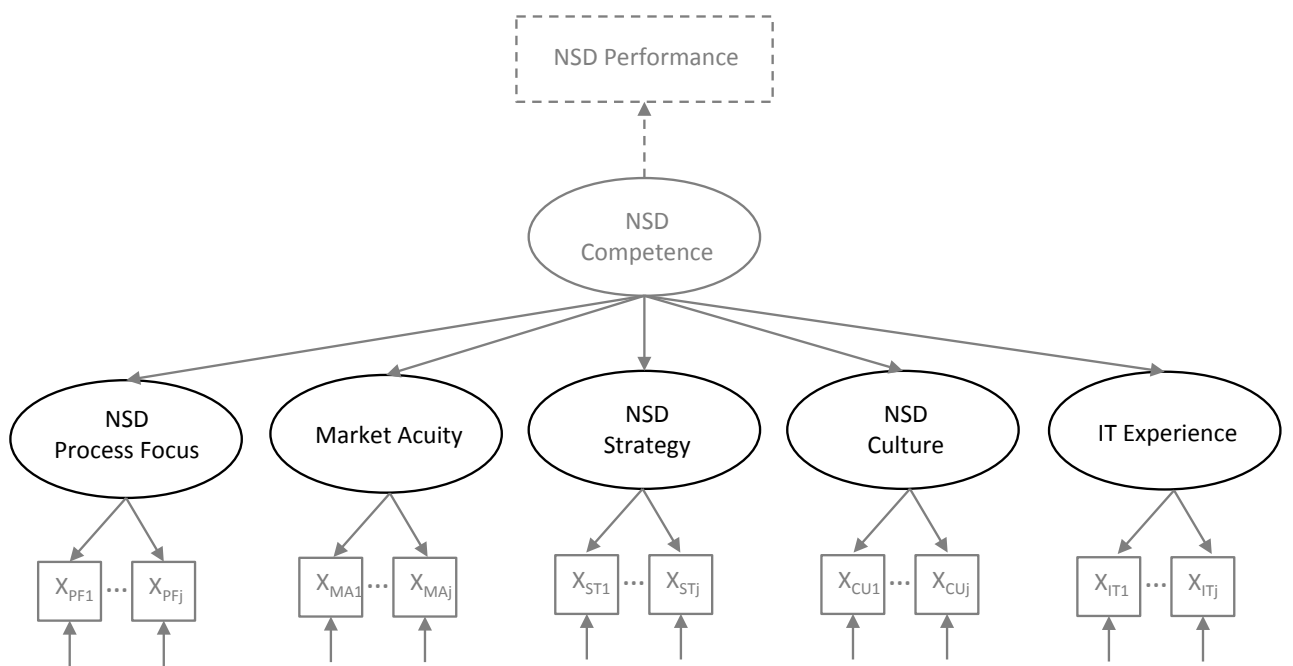

Figure 3.1 Conceptual model of NSD competence dimensions (source: Menor \& Roth, 2007, p.827).

In this dissertation, Menor and Roth's (2007) model has been used as a starting point to create and form a conceptual framework of NSD management. Each dimension has been used as a base but has been modified slightly and complemented individually by other factors which have been identified in the literature as being important when developing new service offerings. The model has been expanded in order to offer a more comprehensive and representative set of theoretically important NSD practice dimensions. In the first stage, the framework is not developed from a specific industry perspective; instead, this adjustment will be taken into account in Chapter 4 , which takes a service development perspective in a logistics industry context. In order to achieve greater content and specificity regarding the dimensions mentioned above, the following subsections aim to break down the following 
dimensions: NSD strategy, market acuity, NSD process focus, IT experience and NSD culture, into various aspects to take into account when developing new service offerings. After each subsection, a summary of each dimension will be presented in the form of a Table, including aspects such as requirements, benefits and supporting activities connected to the dimension presented. Furthermore, this chapter will summarise the different NSD dimensions and important aspects found and also relate and discuss the relationships between any pair of the dimensions included, which will result in a conceptual framework regarding NSD.

\subsubsection{NSD Strategy}

The NSD efforts and activities are described in prior literature as having a positive effect on a firm's service development as a result of an overall strategic focus and defined goals (e.g Johne \& Storey, 1998; Johne \& Davies, 2000) and thus appear to be an important factor in achieving NSD success. This in turn leads to the notion of the existence of a defined NSD strategy which captures the firm's strategic focus and intention behind its NSD. Despite the fact that the existence of an NSD strategy has been shown to be a strong key strategic factor in improving NSD performance, Edvardsson et al. (2013) stress that surprisingly little has been written about service development strategy in NSD literature. However, one significant factor often mentioned in the literature is that a new service strategy or development strategy should both be related to the overall business strategy (e.g. de Brentani, 1991; Sundbo, 1997; Menor \& Roth, 2007) and also fit the image of the firm (Johne \& Storey, 1998). Another interesting aspect worth mentioning is the link between the service development strategy and other functional strategies within the firm. Hence, this is nothing that is specifically mentioned in the NSD literature reviewed. Moving on to the strategic literature, Avison et al. (2004) stress the relevance of a strategic alignment between a firm's business and information technology strategies. Moreover, the authors mention potential benefits a firm can achieve through a strategic alignment between these strategies, such as increasing the return on IT investments, facilitating competitive advantage through IS (information system) and also providing direction and the flexibility to react to new opportunities. Thus, it is not illogical to argue that similar benefits could be achieved if the service development strategy is aligned with the business strategy. This raises the question that if each of the functional strategies in the firm is aligned with the business strategy, does this require each of these functional strategies to be linked to, and support, the others? Furthermore, if that is the situation, does a strategic alignment between the business strategy and the different functional strategies, which in turn are linked to and support each other, lead to higher level of achievement of the benefits mentioned above? However, before determining the strategic alignment between the business strategy and the service development strategy, it is pertinent to define what such a strategy might include.

An NSD strategy can be described as a plan that enables management to organise and make available the appropriate resources for specific new service developments efforts (Menor \& Roth, 2008). In addition, the authors also state that having an NSD strategy "contributes to distinguishing a service firm's 'strategic vision' - an understanding of what the firm and its offerings should be." (p. 828). Kuczmarski and Mandolia (2012) describe a new service development strategy as a strategic platform which sets out the conditions and work as a foundation for creating new services. The authors stress the importance of identifying the strategic role of NSD within the firm in order to prevent the risk of random and disjointed service creation. Furthermore, the authors stress that an effective new service strategy should consist of four main elements:

- the new service vision and the strategic roles that new services must play

- the financial growth gap that new services are expected to fill over the next five years 
- screening criteria to be used for moving ideas and concepts through the development process

- a resource strategy that outlines the financial and human resource requirements for successful new service creation (p. 57).

The role top management plays in influencing this, and a clear vision as to where the organisation is heading, are also crucial to the success of NSD efforts (Johne \& Davies, 2000). This, in turn, places demands on top management regarding their approach and willingness to commit resources to NSD efforts, as well as on their consistency of leadership, through the integration of new information and decisions regarding new service development. A clear strategic focus has been shown to be an important determinant of creative contributions by employees (de Jong \& Vermeulen, 2003). This, in turn, requires the service strategy to be documented and broadly communicated within the organisation so that the firm and its employees have a clear view of how to achieve the new service development goals.

Not only should a service development strategy be formulated with the overall business strategy of the firm in mind, it should also confirm a fit with the appropriate resources and required practices of the developing firm (de Brentani, 2001). According to Edvardsson et al. (2013), a service development strategy involves the internal strategic alignment of resources, capabilities and organisational units. This can force service firms to consider whether, for example, the firm's current technological and employee skills and resources are sufficient when developing and introducing new service offerings. Other aspects to take into account may include to what extent existing facilities can be modified and adjusted in order to complement new service offerings, as well as the firm's ability to employ suitable resources for new service development efforts. This may be important from a long-term perspective. Thus it is reasonable to assume that being proactive and continuously undertaking new service development projects will lead to the creation of capabilities which will be needed in the future, and which will then generate more innovative opportunities than competitors.

In addition to providing a fit with the internal resources and capabilities, the service development strategy should also fit into the context of existing services (Johne \& Storey, 1998). In other words, new service offerings should be designed to complement current service offerings and fit into the existing product mix. This not only generates a strategic alignment of the firm's overall product mix but may also generate a positive outcome from an efficiency perspective.

Another aspect mentioned for inclusion in the service development strategy is the value in use in the customer's context (Johne \& Storey, 1998). Managers must emphasise an NSD strategy that focuses not only on the value proposition and the firm's overall strategy but also on customer value creation. The authors also argue that by balancing this with the internal service capabilities, service firms should be able to reduce the number of new services that fail when introduced to the market. Menor and Roth (2008) also argue that this ensures that new service offerings match customer expectations and demands.

In addition, an effective NSD strategy should also ensure that a new service is developed for a clearly identified target market (Menor \& Roth, 2008). This, in turn, raises the question of the role new service development plays for the firm, which it is reasonable to expect to be reflected in the service development strategy. Does the firm strive to gain market share and increase the loyalty of existing customers as well as attracting new ones through its new service development? Are new service offerings being developed only for markets with high 
growth potential or is it crucial for the firm to introduce new service offerings in order to create or maintain a dominant position in the markets in which the firm competes?

Another aspect worth mentioning is whether the service development strategy takes account of, and relates to, the development of different types of new services, as mentioned in 3.1.1 Types of new services, ranging from radical service development in the form of new-to-theworld services to a more incremental development of services in the form of cost reductions. The definitions of what constitutes a new service are, according to Menor et al. (2002), meaningful both from an external and an internal perspective. From an external perspective, this is in the form of determining the appropriate mix of services in the portfolio as well as understanding how customers or the marketplace perceive the new service offering. Relevant factors from an internal perspective might be the degree of change from an existing system in order to meet the need and requirements for the proposed new service offering.

The results gathered from the study made by Ottenbacher and Harrington (2010) show that different types of innovativeness in NSD require different strategies and approaches if they are to achieve success. For new-to-the-world or new-to-the-firm NSD, market factors (such as market responsiveness and market attractiveness), the pre-launch activities and strategic human resource planning and resulting capabilities appear to be critical aspects to be managed during the NSD process. Meanwhile, for more incremental NSD, service product issues (tangible quality and service advantage), determination of market attractiveness and specific organisational factors appear to be crucial to on-going and incremental NSD success.

Based on the prior literature and discussions, the NSD strategy dimension can be summarised as consisting of a number of main aspects such as Definition \& alignment: visions and goals which are clear and well-established, as well as integrated and in line with the overall business strategy; External match: taking customer expectations and demands into account, as well as having a focus on customer value creation; Internal match: alignment of resources, capabilities and organisational units within the firm; Financial aspects: outline of financial requirements for NSD efforts; Dynamic focus: allowing different types of strategies and approaches for different types of services developed.

To summarise the NSD Strategy dimension, aspects that have been highlighted in the literature have been selected and grouped into the following headings: Requirements, Why/Benefits and Supportive activities, see Table 2 below. The Requirements heading involves aspects that an NSD strategy can both take into account and comprise. The Why/Benefits heading refers to a range of advantages in adopting an effective NSD strategy and Supportive activities involves actions to facilitate the adoption of an NSD strategy. 
Table 2 Summarisation of the NSD Strategy dimension.

\begin{tabular}{|c|c|c|}
\hline Requirements & Why/Benefits & Supporting activities \\
\hline $\begin{array}{l}\text { Definition \& alignment } \\
\text { - Clear and defined goals (i.e. gain } \\
\text { market shares, increase existing } \\
\text { customer loyalty) } \\
\text { - In line with the overall business } \\
\text { strategy } \\
\text { - Fit the image of the firm } \\
\text { - Service vision }\end{array}$ & $\begin{array}{l}\text { Increase the understanding of the } \\
\text { firm's service vision and development } \\
\text { of new service offerings } \\
\text { - Facilitate planning and making } \\
\text { resources available for specific NSD } \\
\text { efforts }\end{array}$ & $\begin{array}{l}\text { - Documentation and spreading of the } \\
\text { strategy }\end{array}$ \\
\hline $\begin{array}{l}\text { External match } \\
\text { - Customer expectations \& demands } \\
\text { - } \quad \text { Focus on customer's value creation } \\
\text { - Clear identified target market }\end{array}$ & $\begin{array}{l}\text { Build capabilities in advance that can } \\
\text { generate innovative opportunities in } \\
\text { the future }\end{array}$ & - Top management support \\
\hline $\begin{array}{l}\text { Internal match } \\
\text { - } \text { Alignment of resources, capabilities } \\
\text { and organisational units } \\
\text { - Match existing technical and } \\
\text { employees skills \& knowledge } \\
\text { Outline human resource } \\
\text { requirements } \\
\text { Complement and fit existing service } \\
\text { offerings }\end{array}$ & $\begin{array}{l}\text { - Influence employees' creative } \\
\text { contributions } \\
\text { - Distinguishing the firm's "strategic } \\
\text { vision" }\end{array}$ & $\begin{array}{l}\text { Top management and senior } \\
\text { management willingness to commit } \\
\text { resources }\end{array}$ \\
\hline $\begin{array}{l}\text { Financial aspects } \\
\text { - Financial growth gap expected to be } \\
\text { filled } \\
\text { - Outline financial requirements for } \\
\text { successful NSD }\end{array}$ & $\begin{array}{l}\text { - Reduce risk for mismatching of } \\
\text { product mix } \\
\text { - Avoid random and disjointed service } \\
\text { creation } \\
\text { - Reduce the number of new service } \\
\text { offerings failure }\end{array}$ & $\begin{array}{l}\text { Consistent leadership by integration } \\
\text { of new information and decisions } \\
\text { regarding NSD }\end{array}$ \\
\hline $\begin{array}{l}\text { Dynamic } \\
-\quad \text { Different types of NSD require } \\
\text { different strategies \& approaches }\end{array}$ & & \\
\hline
\end{tabular}

\subsubsection{Market acuity}

Identifying and responding to market needs is often mentioned in the literature as a success factor when developing new service offerings. It has been shown that when developing new services, companies with a strong market orientation perform better in terms of both sales and competition (de Brentani, 1991). Service firms have been regarded as being too exclusively operations-oriented (Easingwood, 1986). However, today's increased competition and rapidly changing market demands stress the need and relevance for service firms to be more marketdriven in their NSD efforts.

Menor and Roth (2007) describe market acuity as "the ability of the service organisation to see the competitive environment clearly and thus to anticipate and respond to customers' evolving needs and wants" (p. 839). In general, actively seeking information about the firm's business environment and improving the firm's market orientation has been considered to be one of the most strategic options in satisfying market needs more efficiently (Alrubaiee, 2013). Market acuity has also been identified as playing a crucial role (Ordanini \& Maglio, 2009) as well as being the most important success factor in NSD (Menor \& Roth, 2008). Market orientation can be seen as the foundation, or point of departure, for NSD (Sundbo, 1997) and has been suggested as the predominant source of ideas for new services (Alrubaiee, 2013). It is valuable since it requires service firms to continuously seek and collect information on both customers' needs and competitors' offerings, capabilities and market possibilities. This information contributes to and facilitates the NSD efforts, and by developing capabilities to use this information, service firms are able to create new services that deliver superior value (Kirca et al., 2005). 
In the literature, it is widely argued that market orientation consists of core aspects such as customer orientation, competitor orientation, inter-functional coordination (Jaw et al., 2010) and market possibilities (Sundbo, 1997). Customers are often viewed as potential and valuable sources of new service offerings ideas and opportunities (Menor \& Roth, 2008), and the knowledge of customers which employees gain through contact either face-to-face or in a technology mediated setting (e.g telephone, internet and/or commercial online services) provides insights into customers' responses, options and changing needs (Froehle \& Roth, 2004). This is helpful in achieving a customer orientation (Jaw et al., 2010), and collecting detailed customer needs facilitates the ability to develop and offer more customised products and services. Collecting information from and about customers is important, but Edvardsson et al. (2013) also discuss the kind of information and how it is collected, and highlight the ability to integrate and use the often ambiguous customer information as being of particular importance throughout the NSD process. Customer orientation requires sales people to understand a buyer's entire value chain (Jaw et al., 2010). Moreover, the authors stress that customer orientation should be advanced in order to understand customer information and respond more quickly to customer needs by providing new service offerings. Conducting market research also allows firms to design new products to fit different customer profiles (de Jong \& Vermeulen, 2003). In their study (2008), Michel et al. encourage managers to invest in competencies in order to better understand the customers' multi-faceted role as buyers, payers and users. Furthermore, the authors also advise managers to segment selected noncustomers based on the resources available to them (e.g. skills, knowledge, time, money, relationships) and find ways to loosen the constraints on becoming customers.

The input and inspiration for a firm's new service development can also be based on collected information and perceptions of competitors' NSD efforts. A number of service industries have for some time identified competitors as a more important source of ideas for new services than customers (Scheuing \& Johnson, 1989). However, there is a risk that taking too much inspiration from competitors will lead to a reactive and defensive development of services (Kelly \& Storey, 2000). Monitoring competitors' strategies in order to either imitate them or create differentiation in areas such as image, quality, design, time and price is viewed as a source of developing new services (Jaw et al., 2010). The potential benefits of this are that a firm's ability to identify, analyse and respond to competitors' strengths and weaknesses may be enhanced and thus enable the firm to offer service offerings that distinguish it from its competitors.

Achieving a more market-oriented approach does not only involve the firm's creation of market intelligence by collecting valuable information, for example from customers, competitors and market opportunities; it also involves the way in which this information is used and this intelligence is spread within the firm in order to respond quickly to changes in the competitive environment. Jaw et al. (2010) stress the importance of cross-functional integration, which places demands on the level of interaction and communication, the level of information sharing and coordination, and the degree of joint involvement in conducting specific tasks that are involved in the development and launch of new services. Furthermore, the authors state that in order to be responsive or strategically flexible in rapidly distributing, for example, customer feedback, a coordinated and synergistic response to that information is required.

The literature also shows that service firms can adopt different approaches and strategies to achieve an effective market orientation. In their study (2009), Ordanini and Maglio present two different dimensions of market orientation, namely responsive and proactive market 
orientation. A responsive approach involves the acquisition and dissemination of information regarding existing customers and products, in order to respond to and satisfy customers' current needs, while a proactive approach extends the focus and contains to identify latent needs, tapping new market opportunities and cannibalising existing offerings. The authors point out that these two approaches can co-exist, but also stress the importance of proactive market orientation in order to achieve NSD success. In line with Ordanini and Maglio (2009), Veflen Olsen and Sallis (2006) present a similar approach to market orientation. However the authors use the term narrow and broad scanning, which can be compared with Ordanini and Maglio's (2009) description of a responsive and proactive approach. The study by Veflen Olsen and Sallis (2006) revealed that narrow scanning had a strong positive effect on profitability through incremental service adaption while broad scanning had a stronger influence on spin-off knowledge. From a strategic perspective, the authors viewed narrow scanning as a part of the natural operational effectiveness of service firms and claimed that the risk of listening solely to customers without exploring new opportunities could threaten long-term vitality. Moreover, the authors stress that it is not an easy task for service firms to gain superiority in broad scanning, although it has the potential for building superior sustainable profits.

To sum up market acuity, the dimension itself, based on the previous review, can be divided into four main aspects, namely Customer orientation, Competitor orientation, Identification of market opportunities and the Ability to use and integrate the collected market information within the firm when developing new services. Table 3 below summarises the relevant factors found in the literature regarding market acuity. The factors connected to the Requirements heading can, in turn, be divided into four main areas of customer orientation, competitor orientation, identifying market possibilities and the ability to use and integrate the collected market information within the firm when developing new services. The headings Why/Benefits and Supporting activities refer to several outcomes and activities that facilitate the market acuity efforts.

Table 3 Summarisation of the Market acuity dimension.

\begin{tabular}{|c|c|c|}
\hline Requirements & Why/Benefits & Supporting activities \\
\hline $\begin{array}{l}\text { - Collect information on customers' } \\
\text { needs and requirements } \\
\text { - Monitor competitors and collect } \\
\text { information on their offerings, } \\
\text { capabilities and strategies } \\
\text { - Constantly search for market } \\
\text { possibilities }\end{array}$ & $\begin{array}{l}\text { - Increase both sales and competition } \\
\text { performance } \\
\text { - } \text { Facilitate the NSD efforts } \\
\text { Be able to create new services that } \\
\text { deliver superior value } \\
\text { - Be able to offer more customised } \\
\text { services }\end{array}$ & $\begin{array}{l}\text { - Mangers must invest in competencies } \\
\text { related to market acuity } \\
\text { - Managers should not only focus on } \\
\text { existing customers }\end{array}$ \\
\hline $\begin{array}{l}\text { - Be selective in what information is } \\
\text { collected and how }\end{array}$ & $\begin{array}{l}\text { - Satisfy market needs more efficiently } \\
\text { - } \quad \text { Respond faster to market changes }\end{array}$ & $\begin{array}{l}\text { - Sellers must understand a buyer's } \\
\text { entire value chain }\end{array}$ \\
\hline $\begin{array}{l}\text { Develop capabilities to use, integrate } \\
\text { and spread this information within } \\
\text { the company } \\
\text { - Need for cross-functional integration } \\
\text { - Development of approaches and } \\
\text { strategies that include both proactive } \\
\text { and reactive market orientation }\end{array}$ & $\begin{array}{l}\text { - Predominant source of ideas for new } \\
\text { service offerings } \\
\text { - Allow differentiation of service } \\
\text { offerings to customers } \\
\text { - Increase the ability to identify, } \\
\text { analyse and respond to competitors' } \\
\text { NSD efforts }\end{array}$ & $\begin{array}{l}\text { Establishment of a coordinated and } \\
\text { synergistic response system for } \\
\text { market information }\end{array}$ \\
\hline
\end{tabular}

\subsubsection{NSD process focus}

Menor and Roth (2007) describe NSD process focus as "the availability and use of systematic service development practices and routines" (p. 839). In addition, an NSD process focus indicates the existence of a formalised process for conducting NSD efforts and also allows for a simplicity and repetition in the NSD process that fosters greater NSD efficiency and 
effectiveness. Much research has identified NSD process as a success factor for NSD performance and recommended establishing a systematic and formal NSD process (Johnson et al., 2000). One reason for the increased focus on NSD process may be the fact that NSD has often been criticised as being an ad hoc process (Menor \& Roth, 2007). This in turn has led to researchers attempting to provide a systematic and formal structure of which process stages and activities can be associated with the NSD process.

Many of the earlier models are linear in their nature and employ a chronological structure, and are often based on the model of the new product development (NPD) process presented by the consulting firm Booz Allen Hamilton in the early eighties. However, these models have gradually been modified over the years, becoming more aggregated and more generic, as well as involving fewer steps and including feedback loops (Froehle \& Roth, 2007).

Another criticism raised by Johnson et al. (2000) is that many NSD models have been developed for a specific industry, which is a consequence of the small amount of empirical work which exists for testing the developed models in another service industry context. As a result, the knowledge of the nature of NSD processes and its stages, and of how these are managed within service firms, is still limited (Alam \& Perry, 2002). In this thesis, the general four-step model proposed by Johnson et al. (2000) is used in order to illustrate and refer to the different main steps in the NSD process, see Figure 3.2. The reason this model has been selected is that it is relatively uncomplicated, it captures the most common process stages and activities mentioned in the literature, and it has not been developed for, nor does it refer to, a specific firm or industry.

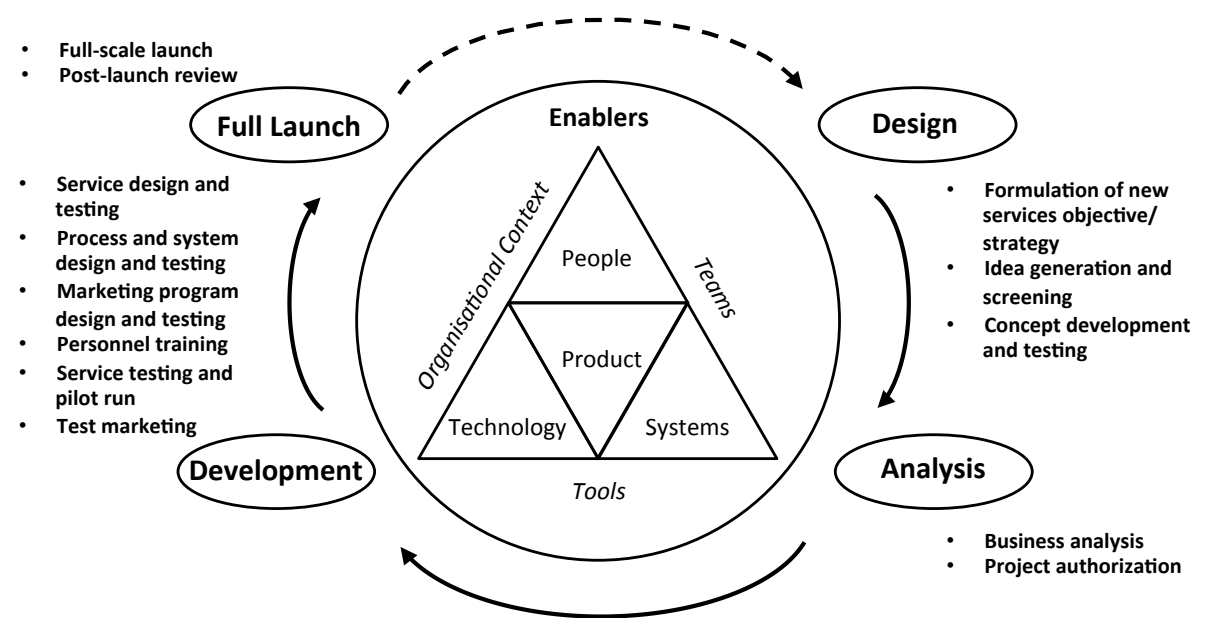

Figure 3.2 The NSD Process Cycle (source: Johnson et al., 2000, p. 18).

The authors divide the different stages into two separate phases, namely planning and execution. The planning phase includes the design and analysis stages, when decisions regarding market viability, internal resources and capabilities are considered. The development and launch stage represents the execution phase, where service delivery system design, use of enablers and cross-functional efforts constitute critical managerial issues for the NSD process.

As mentioned previously, the employment of an NSD process has been seen for some time as a significant element in the development of new services. Despite this, prior research has 
shown that the existing use of a highly detailed NSD process is not common among most service firms (de Brentani, 1991); rather, service firms tend to adopt a more ad hoc NSD process (Johnson et al., 2000). However, the study by Froehle et al. (2000) showed that the formalisation of the NSD process has a direct influence on its speed and effectiveness. Moreover, the authors also suggest that having specific formalised processes for NSD can lead to strategic advantage over competitors in the form of achieving benefits of "first movers" advantages or by more quickly imitating leading-edge competitors.

However, the formalisation of an NSD process as a key success NSD factor can still be questioned. In the study by Menor and Roth (2008), the result revealed that formalised stages and activities in the NSD process play a less significant role in the NSD success than the dimensions of market acuity, new service development strategy and information technology use and experience. This finding slightly contradicts the generally held view that formalisation of the NSD process is most critical for achieving NSD success. However, this does not disregard the relevance of a systematic and formalised process when developing new services. De Jong and Vermuelen (2003) do not recommend that NSD be managed as "something ad hoc". Instead, the authors argue that the NSD should be structured and controlled, although they also stress that the NSD process itself should be managed flexibly. Moreover, the authors point out that service managers should be aware of the effects which a high degree of formalisation of the NSD process might have on the NSD outcome. Since too high a degree of formalisation can have negative effects on creativity and innovation, the authors stress that service managers should strive to find a balance between a highly formalised NSD process and an ad hoc approach. However, there appears to be no suggestion as to what kind of internal process approach is seen as the best alternative to support NSD. Ordanini and Maglio (2009) identify this problem and raise the question "is a more centralised, top-down and formalised process better to leverage internal resources and integrate knowledge compared to a less structured process that involves contact personnel?"

According to de Brentani (1991), service managers responsible for NSD management should establish a system that incorporates "a formal procedure for generating and evaluating new service ideas; a 'drawing-board' approach for identifying and designing the necessary service elements and processes; testing new services with customers and with the frontline in order to eliminate potential fail and points; as well as a documented launch plan to ensure the proper marketing of new services" (p. 55).

An NSD process involves activities and processes as well as information flows which are needed and which facilitate the firm's development of new service offerings. Of course, each step in the NSD process offers a value in itself and provides a function for the NSD process as a whole. However, some steps and enabling factors may be seen as more significant than others. For example, Alam and Perry (2002) view idea generation as the most important step in the development stage of new services and argue, therefore, that service managers should pay more attention to this stage in the NSD process. Besides idea generation, the authors also stress that systematic routines used for screening and selecting NSD ideas, and the existence of cross-functional teams, are also key stages in the NSD process. Another aspect worth mentioning is that the NSP process and its pertaining stages and activities do not also look the same and different NSD projects can each of which require different amounts of resources and level of formalisation. In other words, the NSD process and the activities it includes can be customised based on the different characteristics of the NSD projects adopted by the services firm. This modification of the NSD process may depend on factors such as the characteristics of the particular target market, the newness of the service, competitive 
pressure, the time, resources and investments dedicated to the NSD process, as well as the level of innovation involved (Cowell, 1988). This, in turn, requires service managers to be aware that this may require the NSD process to be adapted, according to what kind of new service is developed. This reasoning is supported by Johnson et al. (2000), who suggest that NSD for radical innovations should differ from NSD for incremental innovations.

Since NSD is often viewed as a highly complex process (Johnson et al., 2000), it is not surprising that a process-focused firm is advised to possess a standard and systematic process for transforming an idea into a new service offering (Menor \& Roth, 2007). Having a standard NSD process with set stages can perhaps facilitate and allow a service firm to be engaged in several NSD projects at the same time to a higher degree compared to the scenario with a non-structured NSD process. Another benefit of a more structured and formal NSD process concerns employee involvement and understanding of the development activities. It is reasonable to believe that having a good understanding of the stages and activities in the NSD process facilitates the process of repeating and improving them if necessary. Furthermore, Johnson et al. (2000) stress the importance of having feedback loops in the NSD process. The authors view feedback loops as critical to NSD efforts, due to the difficulties in analysing the intangible aspects of the service design.

The main aspects of the NSD process focus dimension found in the literature can be summarised by the establishment of systematic and formal NSD practice and routines while at the same time allowing a certain degree of flexibility. It should also allow the existence of feedback loops as well as customisation of the process due to the characteristics of the service being developed. The summarisation of the NSD process focus dimension is illustrated in Table 4 below.

Table 4 Summarisation of the NSD process focus dimension.

\begin{tabular}{|c|c|c|}
\hline Requirements & Why/Benefits & Supporting activities \\
\hline $\begin{array}{l}\text { - Establish systematic and formal NSD } \\
\text { practice and routines } \\
\text { - Allow to be managed flexibly } \\
\text { - Allow customisation of the NSD } \\
\text { process due to characteristics of the } \\
\text { NSD project adopted } \\
\text { - Existence of feedback loops in the } \\
\text { NSD process }\end{array}$ & $\begin{array}{l}\text { - Allows simplicity and repetition in the } \\
\text { - } \text { PSD process } \\
\text { and effectiveness } \\
\text { - "First movers" advantages } \\
\text { - Faster imitation of competitors } \\
\text { - Higher degree of engagement of } \\
\text { parallel NSD project }\end{array}$ & $\begin{array}{l}\text { Employee understanding of and } \\
\text { involvement in the NSD stages and } \\
\text { activities } \\
\text { - Service managers should ensure that } \\
\text { NSD process fits the characteristics of } \\
\text { the developed service } \\
\text { - Service managers should find a } \\
\text { balance between a highly formalised } \\
\text { process and an ad hoc approach }\end{array}$ \\
\hline
\end{tabular}

\subsubsection{IT Expertise}

Information technology has long been seen as the key technology to make services more costefficient (de Brentani, 1991) and it has evolved as one of the most important infrastructural elements of service firms (Froehle et al., 2000). Menor and Roth (2007) refer to IT expertise as "the use of IT for facilitating or improving inter-organisational coordination of activities and information processing in the NSD process" (p. 842). In other words, IT expertise can be seen as a system or tool (Froehle et al., 2000) which enables the creation of services that are more responsive to customer needs (Menor \& Roth, 2008).

It is highlighted in the literature that IT provides a range of possibilities for supporting the NSD process; for example, IT can be an effective tool for better information sharing (de Jong \& Vermeulen, 2003) as well as working as a facilitator for both inter-organisational and intra- 
organisational coordination of NSD activities. Using advanced information technologies, such as groupware, intranets and electronic commerce, can facilitate the process when service firms direct, organise and revitalise flows of this organisational knowledge. The outcome of this can lead to the creation of better NSD processes as well as of better service offerings (Froehle et al., 2000). However, this requires the creation within service firms of information systems capable of enhancing communication among staff as well as with suppliers, partners and customers (Froehle \& Roth, 2007). Through IT, service firms can systematically gather information on competitors' services (de Jong \& Vermeulen, 2003), identify and diagnose customer needs and create databases with customer-related information that can be used in the planning process of NSD activities (Menor \& Roth, 2007).

Another aspect worth mentioning, particularly with regard to information-intensive services, is the role of IT in the production and delivery process of NSD; that is to stress the relevance of the aspects mentioned by Froehle and Roth (2007) in order to maintain back-office and administrative IT systems that can support the firm's NSD efforts and ensure that the IT systems used when developing new services are compatible.

As previously mentioned, there appear to be some suggestions and evidence in the literature regarding IT's ability to support NSD, which Froehle et al. (2000) summarise in three ways: "1) IT enhances communication, reduces communication, reduces information lags and generally speeds up the product development cycle; 2) IT fosters the conceptualisation and creation of new ideas by improving the availability of new content; and 3) IT provides measures for timely organisational feedback, which improves the overall effectiveness of the innovation" (p.6).

The perception that IT system and information technologies have the ability to accelerate the implementation of new services is mentioned by several authors (e.g. Froehle et al., (2000); de Jong \& Vermeulen, 2003). In the study by Froehle et al. (2000), IT choices appeared to play a significant role in improving both the speed of the NSD process and the effectiveness of the firm's NSD efforts. According to the authors, investments in IT systems can increase the generation of new ideas, accelerate the development of new services based on those ideas and in general help the firm to improve the speed with which new services reach the market. However, the benefits mentioned do not happen by themselves. Service firms may question what kind of resources and skills are needed in order to make effective use of IT tools and systems. For example, the study by de Brentani (1991), referred to the problem that there appears to be poor understanding among marketing personnel regarding the potential that information technology offers for creating completely new and innovative services. Consequently, training, employee attitudes and the perception of managerial encouragement have been shown in the literature to affect the effectiveness and adoption of new IT tools (Froehle et al., 2000). Furthermore, the authors stress the importance of IT both in the NSD process as well as its important role in the design and production of the service developed, and argue that managers should take advantage of existing information technologies in order to improve the speed and effectiveness of the firm's NSD efforts.

Based on previous discussion, the summarisation of the IT expertise dimension can be described as including main aspects such as facilitator for information sharing and communication among staff, customers and suppliers to support NSD activities as well as an important factor in the design and production phase of the new service developed. Table 5 below reveals requirements as well as the benefits and supporting activities connected to the IT expertise dimension. 
Table 5 The summarisation of the dimension IT Expertise.

\begin{tabular}{|c|c|c|}
\hline Requirements & Why/Benefits & Supporting activities \\
\hline $\begin{array}{l}\text { - Creation of IT system that capable of } \\
\text { supporting communication among } \\
\text { staff, customers, suppliers } \\
\text { - Maintain existing IT system to support } \\
\text { NSD activities }\end{array}$ & $\begin{array}{l}\text { - Achieve better information sharing } \\
\text { Facilitate for inter- and intra- } \\
\text { organisational coordination of NSD } \\
\text { activities } \\
\text { - Collect information about } \\
\text { competitors } \\
\text { - Systematically gather information } \\
\text { about customer needs } \\
\text { - Facilitate the planning process of NSD } \\
\text { activities } \\
\text { - Improve the speed of the NSD } \\
\text { process }\end{array}$ & $\begin{array}{l}\text { - Managerial encouragement } \\
\text { Increase understanding regarding the } \\
\text { benefits of IT among employees } \\
\text { - Managers should be open towards } \\
\text { existing information technologies } \\
\text { Managers should encourage } \\
\text { investment regarding IT }\end{array}$ \\
\hline
\end{tabular}

\subsubsection{NSD Culture}

According to Menor and Roth (2007), a new service development culture "captures the values and beliefs fostered by the service organisation that indicate a willingness and desire to innovate" (p. 841). The authors also state that the creation of a positive NSD culture that facilitates a climate for NSD activities is essential in order to achieve NSD success. In a similar vein, Jaw et al. (2010) argue that the capability of a firm to create NSD performance depends on its environmental, organisational and innovation context. In other words, the existence of a positive NSD culture can be viewed as reflecting a firm's way of working both internally and externally. The following subsection will present a number of aspects mentioned in the literature as playing an essential role in NSD. These aspects may be affected by the adopted NSD culture of the firm. Thus, the firm's NSD culture may play a significant role in the impact these aspects may have in the development of new services.

In order to create a positive NSD climate there will be a need for different kinds of resources connected to the service firms. Froehle and Roth (2007) mention organisational resources in the form of the management system, attitudes and personnel relationships adopted and developed by the firm as being vital to the firm's NSD process. These are manifested in the firm's development structure, corporate culture, communications norms and top management support. The direct involvement of top management support (Menor \& Roth, 2007) as well as consistent encouragement from senior management (de Jong \& Vermeulen, 2003) is mentioned in the literature as an essential factor in the successful outcome of NSD efforts. Another resource is the employees, who are often seen as the service firm's key resources. According to Froehle and Roth (2007), this resource can capture educational, cultural and experiential knowledge and skills held by the firm's employees. Managers should offer employees training and education connected to NSD efforts. De Jong and Vermeulen (2003) highlight the relevance of improving knowledge and skills regarding trends and developments in a firm's basic technologies, customers and delivery process. The authors also state that this kind of knowledge is vital to the effective implementation of new services and that it influences the success of innovative services, concepts, new client interfaces and delivery systems. In addition to training and education, managers should also encourage and support employee capabilities to work effectively in cross-functional NSD teams (Froehle \& Roth, 2007) and promote information-sharing among co-workers (de Jong \& Vermeulen, 2003). This appears to benefit the firm's NSD efforts by providing better support for the exchange of options and ideas (de Jong \& Vermeulen, 2003), and for the improved creativity and breadth of ideas that are associated with diversity (Froehle et al., 2000). For example, the involvement of frontline employees (responsible for sales and service delivery) and their knowledge of customers and competitive offerings are mentioned as a success factor (e.g. de Brentani, 
1991; de Jong \& Vermeulen, 2003). Thus, according to a study by Melton and Hartline (2010), frontline staff are best utilised in the full launch stage rather than being involved in the idea generation stage. The expertise of frontline employees has a direct effect on customers' perception of service quality (Ottenbacher \& Harrington, 2010) and their involvement in the full launch stage assures expertise in the firm's new service offering (Melton \& Hartline, 2010).

However, using a multi-disciplinary team may not always come without a problem. Vermeulen and Dankbaar (2002) stress that there is no guarantee that members will collaborate successfully, since each individual brings the traditions and work disciplines of their own department. Moreover, a multi-disciplinary team structure cannot guarantee a positive influence on the speed of the service development (Froehle et al., 2000).

Even if identification of internal resources and encouragement of collaboration among involved functional units in the service firm is important, a solely internal focus is not enough in the context of NSD. By broadening the perspective, service firms can gain new knowledge and create a superior service offering through collaboration with other firms offering different operant resources and with customers offering their own operant resources (Michel et al., 2008). Potential collaboration partners include suppliers, institutions, competitors and customers. Benefits that are mentioned in the literature as arising from networking and collaboration with external partners include a reduction in both cost and uncertainty linked to the NSD process, information sharing, acquisition of external knowledge and introduction to new markets (Ordanini \& Maglio, 2009).

Schleimer and Schulman (2011) stress that collaboration with external partners is vital for NSD success. This is due to the dynamic nature of service innovation, which forces service firms to quickly adjust service features and respond to the changing needs and expectations of customers. Because customers' preferences change rapidly, service managers should adopt a more customer-oriented approach to NSD, in order to ensure integration of customer input in the NSD process (Alam \& Perry, 2002). It has been known for some time that collaborating with customers and involving them in the NSD process has a positive influence on NSD efforts. Involving customers appears to benefit services by giving them a greater potential and also provides an opportunity to learn about their latent needs (Matthing et al., 2004). Clear customer inputs have been shown to have a positive influence on the idea generation, service design and service testing stages (Alam \& Perry, 2002) and therefore facilitate the process in the development stage (Melton \& Hartline, 2010). This implies that service firms which adopt a more customer-oriented approach and listen solely to customers are at risk of limiting strategic options for new service offerings, since customers sometimes have difficulty in articulating their latent needs (Veflen Olsen \& Sallis, 2006).

The key to success in providing unique and differentiated services appears to depend on the firm's ability to combine and manage activities and resources linked to the NSD efforts. Moreover, and not surprisingly, new services are generally seen as requiring more support from a firm's organisational structure such as human resources development, process definition and management support (Menor \& Roth, 2007, 2008). In order to see the whole picture, Syson and Perks (2004) argue for, and show the value of, taking a network perspective towards the service development process. Broadening the perspective helps to identify the actors involved, articulate the nature of resources and facilitate and increase the understanding of the development of relationships. Furthermore, the result of the study also reveals that the range of actors, the extent of iteration in the process and the activities 
undertaken might depend on the level of newness of the service developed. For example, the authors argue that more radical innovation may require access to different types of resources with different properties than incremental innovation. In other words, the more innovative the service is, the higher the requirements for the combination of more complex and valuable resources, which may, in turn, require multi-faceted networks of a wider range of actors.

The NSD culture dimension can be summarised as involving main aspects such as having a holistic perspective on NSD, involvement and support from top management, mapping and identification of internal resources and knowledge linked to NSD efforts, existence of crossfunctional NSD teams as well as collaboration with external partners (e.g. customers and suppliers). The requirements, achieved benefits and supporting activities connected to the NSD culture dimension can be found in Table 6, below.

Table 6 Summarisation of the NSD Culture dimension.

\begin{tabular}{|c|c|c|}
\hline Requirements & Why/Benefits & Supporting activities \\
\hline $\begin{array}{l}\text { - Existence of cross-functional NSD } \\
\text { teams } \\
\text { - Mapping and identification of internal } \\
\text { resources linked to NSD efforts } \\
\text { - Collaboration with external partners } \\
\text { (e.g. customers, suppliers) } \\
\text { - Adopt a more customer-oriented } \\
\text { approach to NSD } \\
\text { - Have a holistic perspective on NSD } \\
\text { - Direct involvement of top } \\
\text { management and senior management } \\
\text { support }\end{array}$ & $\begin{array}{l}\text { Better support for exchange of } \\
\text { options and ideas } \\
\text { - } \text { Improved creativity and breadth of } \\
\text { ideas } \\
\text { - Gain new knowledge and resources } \\
\text { through external collaborations } \\
\text { - External collaboration may lead to } \\
\text { reduction in cost and risk in the NSD } \\
\text { process } \\
\text { Customer involvement leads to direct } \\
\text { and clear input of their needs and } \\
\text { demands } \\
\text { Help to identify actors and resources } \\
\text { needed for NSD }\end{array}$ & $\begin{array}{l}\text { Offer employees training and } \\
\text { education connected to NSD efforts } \\
\text { Promote information sharing and } \\
\text { cross-functional working }\end{array}$ \\
\hline
\end{tabular}

\subsubsection{A conceptual framework for management of NSD}

As mentioned earlier in Chapter 3, this section aims to discuss and link the different NSD dimensions that have been presented above in order to form these dimensions into a conceptual framework. The literature regarding NSD appears to show a need for a better understanding of strategic management of NSD (Menor \& Roth, 2008). Moreover, there appears to be an understanding among service firms regarding the potential benefits of taking a broader perspective of NSD (Froehle \& Roth, 2007). It is perhaps necessary to broaden the perspective in order to understand NSD and how it should be managed, since achieving a successful NSD will depend on a firm's ability to create skills and competences in the management of service development resources and organisational routines (Menor \& Roth, 2008).

The question remains, however, of how service firms should act in order to achieve a more strategic and holistic approach regarding their service development management? As mentioned earlier in this chapter, no best practice appears to have been identified regarding how service firms should manage their NSD activities in order to achieve maximum output from their efforts. However, each of the NSD dimensions presented in this chapter has been identified in the literature as being important and having a strategic influence in order to succeed in the development of new services. It is not unreasonable to believe that these dimensions all play different roles and have different levels of influence and importance in NSD. Many studies in the service development and innovation literature have examined and identified the individual effect of critical success factors in NSD. Menor and Roth (2008) argue that, theoretically, what matters is the co-variation or complementarity among the NSD 
dimensions that form and create competences regarding service development. Therefore, Menor and Roth (2008) view the studied NSD dimensions in their framework as mutual complements. Likewise, Ordanini and Maglio (2009) also stress in their study that the studied dimensions are not separate entities, but rather that the dimensions should be seen and handled as intimately connected elements of a service system. Therefore, in order to examine the effectiveness of service development, Hull (2004) argues the need to investigate the joint impact of NSD practice and activities. This is plausible, since NSD conduct a system of development practices and routines which are related to each other, in order to influence a firm's ability to effectively develop new services (Menor \& Roth, 2008).

The discussion regarding how to clarify the role and influence of the different NSD dimensions starts with the NSD culture. The presentation of the dimension in 3.2.5 took up relevant aspects such as employee knowledge and attitude, internal resources, existence of cross-functional teams, management involvement and support as well as the attitude towards external collaboration with, for example, customers, supplier and competitors.

These aspects are to a large extent related to the firm's organisational structure and culture and can be viewed as a base that offers and sets pre-conditions for the other NSD dimensions. The NSD culture dimension can thus be argued to have a supportive role, and Menor and Roth (2008) state that without support from internal capabilities, structures and a supportive service culture, it can be difficult to achieve NSD success and market performance. For example, Ordanini and Maglio (2009) show in their study that integration with external partners in the market orientation efforts can help to reduce costs and give valuable input, and so lead to successful new services. Moreover, both collaboration with other partners, as well as cross-functional teams, can be seen as learning-based input to the NSD.

There is also a need for strategic conditions to be created, given that strategic intent appears to have an influence both on the selection and success of approaches when developing new services (Hull, 2004). Menor and Roth (2008) state that NSD strategy defines the goals and leadership, which indicates that NSD strategy can be seen to have some kind of controlling and guiding role in NSD. The NSD strategy sets the rules for NSD based on the condition of the service firm as well as sets the goal of where the firm with its NSD is heading and what and how to do in order to achieve it. This central role implies that the NSD strategy will, in all probability, influence and place requirements on the market acuity, process focus and IT expertise when developing new services. The controlling role of NSD strategy and the structural role of NSD process focus is mentioned by Edvardsson et al. (2013), who stress that these two dimensions "should enable and secure external and internal learning and the integration of different skills and customer knowledge in the right stage of the service development process" (p. 38). An NSD process can work as a structured means in order to implement NSD within a firm (Menor \& Roth, 2008). As mentioned previously, a too highly formalised NSD process can act as a barrier to NSD and harm the creativeness among employees. Furthermore, in the study by Menor and Roth (2008), NSD process was shown to have a less significant role in defining NSD competence compared to the market acuity, NSD strategy and IT experience dimensions. In a similar vein, Froehle et al. (2000) also stress that NSD process formalisation seems to have a more indirect impact on both the effectiveness and speed of NSD efforts. Despite this, the authors, as well as other researchers, claim that an indicatively and formalised NSD process is necessary and should thus allow a certain level of flexibility. Regarding IT expertise, this dimension was shown in the study by Menor and Roth (2007) to be highly related to NSD process focus and market acuity and thus viewed as an important component in the portfolio of NSD resources. Based on the prior presentation of IT 
expertise, it can be concluded that this dimension can both have a role as an enabling tool when it comes to sharing information and facilitating communication and also have a technological role in the design and creation of the new service.

The market acuity dimension was found in the study by Menor and Roth (2008) to be the core of the NSD dimensions studied. Market acuity in the form of creating knowledge about customer needs and market trends and the ability to integrate this information when developing new services can therefore been seen as relevant and as having a role as an input of knowledge and skills into the development of new services.

Furthermore, Menor and Roth (2008) state that "leveraging employees' first-hand knowledge, skills and involvement with customers to identify opportunities for developing innovative service offerings or delivery processes may be an important antecedent to market acuity" ( $p$. 279). This shows the relevant role of front-line employees as well as customer integration and collaboration as an essential input of knowledge and skills into NSD. These aspects, together with market acuity, cross-functional teams and collaboration with external partners, all relate to knowledge and skills reflecting issues such as what knowledge to capture and how. The term Knowledge and skills will hereafter be used as a NSD dimension, instead of the label Market acuity. The dimensions including aspects is regarded to act as input that feeds the development of new services and can bee seen as core to NSD.

Based on previous discussion and by taking a holistic and strategic approach to NSD, this leads to the following conceptual framework of NSD management, see Figure 3.3 below. The following conceptual framework can be seen as a logical way to view NSD and its included dimensions as well as the connection between these and the role these dimensions play.

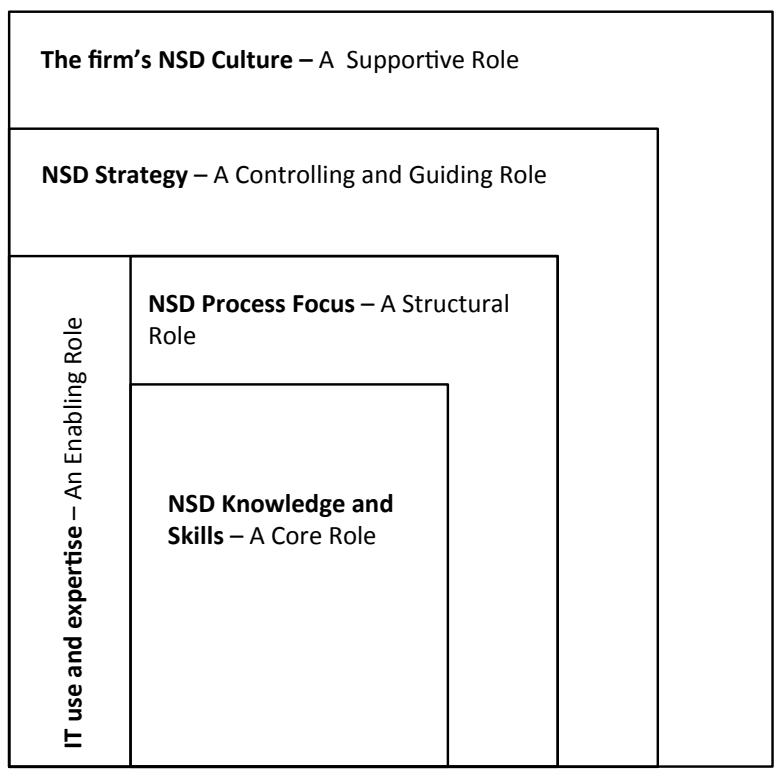

Figure 3.3 A conceptual framework of NSD management.

In sum, in order to clarify the conceptual framework, it can be described to consist of several layers. The first layer symbolises a company's NSD culture with its supporting role, which can be seen as a foundation which upon the other mentioned NSD dimensions build. The 
second layer in the framework concern the central position of the NSD strategy dimension that with its controlling and guiding role has an influencial impact on the rest of the NSD dimensions in the framework. Next, upon the NSD culture and NSD strategy layers comes the NSD process focus dimension with its structuring role to support activities, knowledge sharing well as to integrate skills and customer knowledge into the different stages of the service development process. The top layer of the framework represents the dimension labelled as NSD knowledge and skills, which is seen as the core that feeds the development of new service offerings. As mentioned before this dimension includes aspects such as employees' knowledge and skills, customer- and market knowledge as well as knowledge and skills from external partners. The last NSD dimension in the framework concerns IT use and expertise and its vertical position can be explained by its enabling role as an important resource connected to the dimensions NSD process focus as well as NSD knowledge and skills. 


\section{CHAPTER}

\section{THE LSP CONTEXT OF NEW SERVICE DEVELOPMENT}

The main aim of this chapter is to identify and compile the status of LSPs' service development in prior research. First, there is a short introduction to the LSP industry as well as some market trends that have influenced this industry during the years. This is in order to better understand the relevance and possible opportunities LSPs can obtain by focusing on the management of NSD. After the section concerning the need for NSD within the LSP industry follows a discussion of different NSD dimensions LSPS should consider in their further NSD efforts. The outline of this discussion follows the same structure as presented in Chapter 3. This chapter ends with a summary of these NSD dimensions and their connection to each other in order to identify the overall importance for LSPs' management of NSD. This discussion results in some survey questions, intended to guide the further empirical investigation. 


\subsection{Description of the LSP industry/market trends}

In general, the LSP market can be described as consisting of a huge variety of players which differ in terms of size, geographic coverage and service offerings (Prockl et al., 2012). In addition to increased globalisation and an increased number of new players, the logistics market has also been influenced by the rapid development of IT and the growing awareness regarding environmental effects on society. More specifically, the development of the logistics market, as well as of its actors, can today be said to be influenced by the following factors:

- Increased globalisation and growth in global trade - the general economic growth as well as increasing global trade creates and drives an increased demand for logistic services.

- Digitisation - an increasing communication capability has a positive effect on international trade and thus enables growth in e-commerce.

- Changing customer behaviour - demand is increasingly controlled by customers, with a greater focus on flexibility and precision in delivery time, information on the logistics flow, safety as well as cost.

- Internationalisation - globalisation drives an increasing need for more complex and border-crossing logistics solutions. This places requirements on international reach and management for more efficient information technology systems within logistics.

- Regionalisation - due to increasing international trade and open borders, the capacity of regions will have greater importance for logistics operations than the capacity of a single country.

- Environmental requirements - greater awareness and pressures from society and customers to reduce environmental impact from transportation to contribute to climate change. (PostNord, Annual Report, (2011), p. 24)

Logistics services have been developed in both knowledge and scope in response to different market trends and forces (Hertz \& Alfredsson, 2003) and logistics management has been identified as an important source of competitiveness (Chapman et al., 2003). Logistics can be said to "have evolved from being a tactical requirement into a strategic activity that links customers and suppliers by managing the flows of goods, services and information from point of origin to point of consumption" (Mena et al., 2007, p. 3). In addition to transport and warehousing, other common activities within logistics are forwarding, customers' clearance, packaging, labelling and information handling systems (Jumadi \& Zailani, 2010). The logistics industry can also be said to be "a classic example of the birth and development of a vital new service-based industry, transformed from the business concept of transportation to that of serving the entire logistics needs for customers" (Chapman et al., 2003, p. 1). This puts pressure on LSPs to fully understand the whole supply chain of their customers in order to develop and provide customer-oriented logistics solutions (Pekkarinen \& Ulkuniemi, 2008).

Those LSPs who want to create a position of competitive advantage must take on this challenge in order to meet these different requirements. The changing transportation and logistics markets have forced LSPs to adjust their business models to provide a higher share value to customers (Third-Party Logistics Study, 2012). One way to stay competitive and take on the opportunity for long-term profitable growth suggested in the literature is the development of new services and investments in logistics innovative efforts (e.g. Soinio et al., 2012; Wagner \& Franklin, 2008). The role of innovation and its increasing focus within the 
LSP industry can also be illustrated by de Souza and Goh (2008)'s description of the LSP industry:

"The logistics industry serves as a good example of the genesis and growth of an essential, new innovation-driven and value-recognised service-based industry, transformed from the traditional business notion of simple point-to-point transportation to that of serving the entire suite of customers' logistical needs" (de Souza \& Goh, 2008, p.62).

There now follows a description of the status and need for NSD within the LSP industry. Innovation is in this dissertation seen as part of NSD. Therefore, some examples of logistics innovation definitions are presented as well as how this term is used in this dissertation.

\subsubsection{Definition of logistics innovation}

In common with many other concepts and terms, there is a variety of definitions of logistics innovation in the literature. In the study by Mena et al. (2007), the authors divide logistics innovation into two groups, namely technical/technological and administrative/non-technical. The first group concerns technologies for data acquisition, information administration, warehousing and transportation, while the second group concerns organisational structure and processes, business activities and its management. In Wagner's (2008) definition, on the other hand, logistics innovation is divided into product/service versus process innovation. A product/service innovation occurs when a customer is provided with a new or improved service that meets the new performance demands, while a process innovation involves implementation of new or improved techniques, methods or process in order to reduce cost or improve the quality of the service provided. However, the author highlights the importance of finding a balance of both these types of innovation in order to manage different challenges and complex demands from the market. Flint et al. (2005) suggest a broader definition of logistics innovation that is not restricted to products or technology alone. The authors define logistics innovation as "any logistics related service from the basic to the complex that is seen as new and helpful to a particular focal audience" (p.114). By audience, the authors mean that the innovation can benefit the company internally or externally towards its customer. For the purpose of this dissertation, the definition of Flint et al. (2005) is adopted and is in line with the authors' focus of interest, which is to concern a more external audience.

\subsection{The status of and need for NSD within the logistics industry}

Based on the literature, it can be stated that service development and innovation management have not received much attention in prior logistics research (e.g. Flint et al., 2005; Busse, 2010). Similar scenario can found in the business world, where innovation management seems to receive a minor role among many LSPs (Wagner \& Sutter, 2012).

Trends indicate that the outcome from LSPs' innovation efforts is generally lower compared to other industries (e.g. Mena et al., 2007; Wagner \& Franklin, 2008; Wagner \& Sutter, 2012). A possible explanation for this, given in the literature, is the mature phase of transportation and logistics industry, where change is evolutionary rather than revolutionary (Mena et al., 2007). Another reason mentioned is that new service initiatives often take place in collaboration with specific customers, which hampers the dissemination of lessons learned and knowledge regarding its development throughout the company (Wagner \& Franklin, 2008). In addition, Busse and Wallenburg (2011) argue that the majority of logistics improvements are driven by LSPs' customers, and suggest that this trend regarding 
customers' requirements for LSPs to develop new and innovative services will increase over time.

The transport and logistics industry, as mentioned earlier, is not known for being the most innovative industry. A study by Busse (2010) revealed that utilisation of R\&D is noticeably lower for innovative-active LSPs than for innovative-active non-LSPs. Suggested explanations for this situation are that the LSP industry is strongly characterised by imitations and that technology is adopted rather than generated by LSPs. For these reasons, the author presumed that LSPs strive for less radical novelties than non-LSPs and both the need for new knowledge and investment in R\&D activities seem to be low. The results also indicated that it seems to be more costly than for non-LSPs; the author thus suggests that LSPs should pay more attention to the efficiency of their innovation projects as well as to payback of their innovation-related investments.

Another example of LSP innovativeness can be found in the study by Kuester et al. (2013), in which the LSP industry was labelled as an efficient developer. The study aimed to investigate how innovation activities vary among different clusters of the service industry and how various success factors are perceived. An efficient developer is characterised by a low adoption of implementation of externally developed innovations; generation of external knowledge generally has low importance as well as low existence of integration with customers or other external sources. Furthermore, according to the study by Kuester et al. (2013), an efficient developer focuses more on standardisation of their services, trying to reduce costs, and due to the similarity with competitive offerings, differentiation is a challenge and difficult to achieve. Due to the low integration of external partners when developing new services, an efficient developer is particularly dependent on the market's initial response. Thus, Kuester et al. (2013) stress that an efficient developer must focus on the quality of the service experience, the superiority of the service, customer orientation and internal cooperation in order to be successful.

This description is a broad reflection of the way service development may look among LSPs today. However, operating in markets that are typified by variations in customer characteristics and increasing customised demands, as LSPs do, raises a need to provide more than standardised services. Focusing solely on standardisation of its services is perhaps not a sustainable solution in a long-term perspective when some LSPs have high growth demands and also increasing demands to provide more customised solutions to their customers. In addition, the relevance of a differentiation of logistics services in order to reach an optimal balance between customer satisfaction and cost levels for the services provided is a wellknown fact for LSPs (van der Veeken \& Rutten, 1998). Thus, there is a need for the LSP industry to make the move from being a production-oriented industry to becoming more customer or market-oriented in their NSD efforts in order to keep up with changing market trends and meet the diversity of demands that arise in the market. By doing this, LSPs may face challenges in finding the right balance between high adaptation ability to an individual customer and the coordination of several customers (Hertz \& Alfredsson, 2003). However, Wagner and Franklin (2008) state that inspiration can be drawn from the professional service firms (such as management consultancies) and how these firms capture value from new services provided to their customers. These companies have built up a capability to capture the knowledge acquired by their field teams, identifying trends or solutions and then repackaging this information in order to create specific customer solutions. By collecting, analysing and packaging this knowledge into solution frameworks, they increase their ability 
to deliver solutions that are customised for a specific customer but that also support their own strategic focus.

Several benefits and reasons regarding why LSPs should start concentrating on, and investing in, NSD efforts are mentioned in the literature. For example, it can be seen as a way to differentiate LSPs from their competitors (e.g. Flint et al., 2005; Wagner, 2008) and may also improve the perception of being a trustworthy actor (Wallenburg, 2009). Furthermore, new service development can also be seen as way for LSPs to achieve increased growth. Due to the fact that the logistics industry is no longer seen as an emerging market, LSPs must rely on growth strategies such as taking market shares away from competitors and strengthening existing customer relationships, both of which, according to Wagner and Franklin (2008), can be achieved by developing and providing new service offerings.

According to Wallenburg (2009), the main motivation for LSPs to evolve innovative initiatives and develop new services concerns firstly to improve service quality, secondly to reduce cost and thirdly to improve existing customer relationships. Further, the authors state that improving existing customer relationships is more cost beneficial than gaining new ones and can lead to positive outcomes such as increased customer loyalty, as well as expanding the relationship between an LSP and its customer.

However, in order to understand how LSPs can achieve and gain from these opportunities there is also a need to understand the obstacles that LSPs may face when they develop new services. According to Zhou and Wang (2012), most LSPs lack the expertise and skills required for new service development and have limited knowledge of the process of new service development. As a result of this, Wagner and Franklin (2008) state that LSPs often struggle to extend customised solutions so they can be adapted to a broader customer base. Similarly, Oke (2008) says that there is a mismatch between time spent on responding to customers' immediate needs and the time spent on more long-term innovation efforts. Other identified barriers mentioned in LSPs' innovation management are: "lack of a clear definition of innovation, ineffective knowledge transfer, lack of effective research and development processes, difficulty of testing concepts due to the intangibility of logistic services and difficulties to protect innovations with patents" (Oke, 2008).

It has been suggested that innovation is, and most likely will continue to be, a strong competitive advantage for LSPs in the coming years (Oke, 2008). However, LSPs must be aware of the fact that a logistics service that is highly valuable today may not be sufficient for customers in the near future (Flint et al., 2005). Moreover, whether or not an improvement to existing logistics services or the development of new ones is perceived as successful will primarily depend on LSPs' knowledge of what their customers value (Wallenburg, 2009). This raises a need for LSPs to develop their ability to look for market information as well as their skills in using this information when developing new services. In addition, Wagner and Franklin (2008) argue that without a systematic management or structure for innovation activities in general, LSPs will not be able to gain the full benefit of these efforts.

The discussion above raises the question of what can be done in order to improve and increase the capability for NSD among LSPs. It is clear that there is a need for theoretical development and understanding of NSD in the context of LSPs. 


\subsection{NSD and its management among LSPs}

The following sections aim to investigate the status of LSPs' service development more deeply, and are structured around the different dimensions in the conceptual framework of NSD management presented in section 3.2.6. This is in order to identify key areas and aspects related to these dimensions, as well as other aspects, in order to increase the understanding of LSPs' management of new service development and innovation activities.

\subsubsection{The NSD Culture of LSPS}

The literature review for this dissertation has revealed little regarding the NSD culture of LSPs. However, this may well be a result of the ambiguity regarding what constitutes a company's NSD culture as well as difficulties in creating such a culture. Nevertheless, it should perhaps not be neglected, since it provides a basis that both offers opportunities and sets boundaries for a company's ability and attitude to develop new service offerings.

According to Busse and Wagner (2008b), an innovation culture should provide an environment that fosters and shows awareness of innovations. The authors also mentioned the establishment of such a culture as an important aspect in their guideline for implementing an innovation management system at LSPs. Likewise, Flint et al. (2005) also include considerations of an NSD culture in their innovation management model for LSPs. The authors define it as "setting the stage" and it can be described as a foundation for managing an innovative organisational culture. This foundation should be modified and refined over time based on previous learning from the company's NSD efforts. Examples of activities connected to this stage might include "creating a customer retreat environment, managing an innovative, customer-oriented culture, hiring and training innovative, customer-oriented personnel, creating systems to capture customer data and restructuring account management teams" (Flint et al., 2005 p. 128).

Furthermore, it is also suggested in the literature that a company's choice of organisational structure may have an effect on its organisational NSD capability. This may, in turn, also have an impact on the creation of the company's NSD culture. For example, Busse and Wagner (2008a) suggest some success factors connected to innovation structure found in their study such as a central coordination centre, hierarchical control of innovations projects and the existence of an interdisciplinary team structure. Daugerthy et al. (2011) on the other hand suggest, on the basis of the results in their study, a positive relationship between decisionmaking decentralisation and innovation capability regarding logistics service innovation as well as how much autonomy and flexibility the company allow employees regarding these issues.

Furthermore, creating and setting the stage for an NSD culture involves, as suggested by Flint et al. (2005) the creation of a creative and supportive environment for employees as well as involved partners to develop new services. However, senior management plays an important role in this effort (Busse \& Wagner, 2008b) and there will be a need for managerial engagement. The supportive role of managers in motivating employees, as well as engaging in dialogue with customers and suppliers, can have significant impact when there is a desire to improve its service development abilities. This is supported in the study by Flint et al. (2005), which emphasised that innovations do not happen without internal commitment from the top management and from other relevant managers within the company. A clear understanding of how both the LSP and its customers would benefit from the proposed new service as well as defined stages for service development process seems to be relevant. 
Another aspect that can help to set the stage and create a culture that facilitates NSD efforts concerns the company's attitude of utilisation of cross-functional teams. Flint et al. (2008) argue that using such teams can facilitate cross-functional learning within LSPs and also enable better clue gathering when developing new services. Chapman et al. (2003) highlight the importance of the existence of organisational structures since these structures constitute important facilitating factors when LSPs develop new services. In addition, the authors especially stress the relevance and creation of inter-organisational structures. This can be supported by the fact that there appears to be a growing need among LSPs for seeking new knowledge and working with customers and suppliers in order to improve their service offerings (e.g. Flint et al., 2008; Shen et al., 2009).

Further, it is suggested in the literature that LSPs should also create a culture that promotes and supports learning from other actors since it is suggested that logistics innovation can be an outcome of organisational and inter-organisational learning (e.g. Flint et al., 2008; Flint $e l$ $a l ., 2005$; Panayides \& So, 2005). In order to create a culture of organisational learning, Panayides (2007) states that "managers must stress the value of learning and facilitate learning by encourage and facilitate employees to work together in achieving common goals, to share information with each other and other departments, to reflect critically on actions taken in serving the customers and performing their duties and to preserve and distribute the knowledge created during the course of their operations" (p.136). In addition, the author highlights the importance of managerial efforts and commitment in order to make this happen in practice, as well as influence issues related to cross-functional integration, identification of customer needs, information processing, production and technology. An accurate collection, management and intra-organisational dissemination of information is necessary to add value in the development process of logistics services and this can lead to better performance by the company. However, Flint et al. (2005) stress that the outcome of organisational learning depends on, and is affected by, the unique structures, organisational culture, national cultures and situations of the specific LSP.

It has also been recommended that LSPs consider expanding the boundaries and including customers and suppliers into a more inter-organisational learning process when developing new services. For example, managers are advised to focus on designing relationship experiences that facilitate dialogue and interaction based on the characteristics of the relationships in order to gain understanding of their customers and what they value (Yazdanparast et al., 2010). However, having a good understanding and knowledge of their customers may not in itself lead directly to innovation, rather this may occur indirectly via organisational learning (Panayides \& So, 2005).

The understanding and sharing of knowledge, including on occasion tacit knowledge, between partners, which in turn can lead to problems in replicating lessons learned with other partners, is seen as a barrier to LSPs' innovation efforts (Oke, 2008). This in turn can support the relevance of LSPs having a stronger learning approach when developing new services, both within their own company and towards external parties.

Furthermore, Flint et al. (2008) extend the concept of inter-organisational learning one step further and refine it as "supply chain management learning". The authors define it as "the degree to which firms look both up and down their supply chains to manage and monitor learning processes within and outside of the firm" (p.264). There is a focus on supply chain solutions and issues which involves a range of supply chain partners who interacted with the aim of increasing learning between these partners. Example of potential initiatives taken may 
involve "product modifications, entirely new products, or supply chain modifications such as network redesign, transportation route and mode modelling, package design, information systems modifications, enhancing goods in transit visibility through the application of emergent technologies or improving forecasting accuracy" (p.274). Furthermore, the term supply chain learning refers to having a better dialogue and finding better solutions to serve each other in the supply chain and the authors recommend that managers of LSP decide to which degree they want to manage or support this kind of learning within their own company. This is necessary in order to secure that employees will learn lessons and take advantages of the opportunities that may arise in such interactions.

Creating and setting the stage for an NSD culture that supports new service development is a challenge; however, the discussion above shows the relevance of managerial support and the existence of an environment that supports employees, cross-functional interaction as well as interaction with other external actors. This NSD culture or company environment should also support and facilitate learning not only within the company but also with other actors in the supply chain. A more comprehensive description of the role of increasing employees' knowledge and gaining external knowledge will be presented in section 4.3.5 together with other knowledge and skills needed for LSPs' service development. The existence of a wellestablished NSD culture may not be enough to improve a company's NSD ability; there will also be a need for some strategic directions regarding these efforts, specifically in the form of an NSD strategy. The next section will explore the existence of such a strategy within LSPs.

\subsubsection{Strategic focus and existence of an NSD Strategy}

The existence of an NSD strategy provides, to some extent, the direction and the focus areas for a firm's efforts to develop new services and seems therefore to play a central role in NSD management. But how is this aspect treated in the literature on LSPs? In their literature review regarding innovation management of LSPs, Busse and Wallenburg (2011) identify articles on strategy, expansion and growth of LSPs, although these do not refer to innovation, and the authors also state that there does not appear to be any single best innovation strategy for LSPs. Therefore, the authors question the relevance of a service development strategy, since LSPs seem to grow without innovating, at least in the short term. However, at the same time, the authors highlight the relevance of innovation as an opportunity to increase the growth of LSPs in the future. Based on previous discussions in section 4.1 regarding the changing business environment as well as customer demands, the relevance of NSD and the existence of such a strategy is perhaps especially important for LSPs to consider not only today but also in the future.

The lack of an NSD strategy among LSPs can perhaps partly be explained by some of the identified barriers found by Oke (2008) in relation to LSP innovation management. The author found that a clear definition of innovation, in other words what it means for the company as well as how it can be managed, seems to be lacking within the LSPs. However, the author stresses that trying to define what innovation means for the firm can help LSPs to focus on the most appropriate innovation areas and efforts of the firm. The choice and coordination of a service development strategy can be regarded to be the centre position among all the different activities related to NSD among logistics (Zhou \& Wang, 2012). Furthermore, the authors stress that NSD should be placed under the LSP's strategic management, and should involve and encourage a positive approach to meet the market challenges. 
Oke (2008) also states the importance of relating innovation to the business strategy. However, in order to concretise the business strategy, Busse and Wagner (2008b) argue the necessity of developing an innovation strategy which supports and connects within other strategies within the firm. In addition, the authors state that the business units should organise the concrete specification of an innovations strategy's content in order to match that specific business unit.

Regarding the content of what an NSD strategy may include, Busse and Wallenburg (2011) divide innovation strategy into two areas, namely strategic orientation and strategic leadership. By strategic orientation, the authors mean areas such as growth and differentiation approaches, innovation management possibilities, knowledge protection and importance of technology, while strategic leadership, which was mentioned in section 4.3.1 in this dissertation, involves behaviour and attitudes of senior management in respect of innovation management. The involvement and support from top management in relation to the innovation strategy was also shown to act as a success factor in the study by Busse and Wagner (2008a). The success factors regarding LSP innovation management were derived from a panel of experts consisting of seven executive managers of four large international LSPs. Other success factors found in relation to the innovation strategy were that the strategy should emphasise a content-related focus, standardisation and allow openness and proactive approaches when searching for new ideas.

By taking a bigger marketing perspective, Speh (2008) expresses the need for LSPs to understand the cornerstones of the marketing strategy as well as how to develop the elements of their marketing strategy in order to be able to execute the strategy. According to the author, developing new services and meeting the customers' needs requires an integrated marketing strategy. The first step is the selection of the needs of the target market, followed by a marketing mix that matches the expectations of each segment. The marketing mix will include key elements such as "service product" or "service package", pricing, promotion and distribution. In addition, the author stresses that all these key elements require a certain focus from the LSP marketing manager in order to create the appropriate conditions for the new service product.

However, one important issue that should not be neglected when developing new services concerns the strategic focus and alignment with the existing service offerings provided by LSPs. Sonio et al. (2012) highlight the importance to LSPs of considering and understanding the options for expanding their business scope as well as resources needed when deciding how to position themselves in order to provide value to their customers. The alternative, having an uncontrolled service development, means LSPs may risk ending up with a portfolio of overlapping services (Wagner \& Franklin, 2008) that may not support the overall strategic business development of the company.

Shen et al. (2009) suggest some examples of objectives for the strategic service development among LSPs, namely modularisation of logistics services as well as a significant reduction of logistics costs. However, the authors argue that this trend will, in all probability, change in the future and cost-oriented logistics service offerings will be replaced by more customeroriented service offerings. Reasoning between the selection of a standardised service approach and a customised approach can be found in the study by Busse and Wallenburg (2013). However, the authors argue that the underlying reasons for this need to be carefully considered since the decision not only affects the management of LSPs' service development but also has an impact on the daily business. 
Moreover, Shen et al. (2009) argue that to achieve strategic NSD, LSPs must in turn adapt, exploit and even create turbulent environment on their own initiatives in order to continually restructure modes of value creation. This in turn can be related to how the company wants to position itself and its services on the market in order to create value to its customers. Flowers et al. (2008) suggest that the decision on how to position themselves on the market will also help LSPs to match their innovation efforts to the strategic choices of the company. The authors present a model, originally based on and inspired of the research of Treacy and Wiersema (1995), consisting of three different market leadership models that should help LSPs to focus on their innovation efforts.

- Operational excellence - high focus on implementing the technology and business process in order to increase operations efficiency.

- Product leadership - the innovations efforts should pioneer original innovations and/or LSP must choose the right mix of capabilities to match a significant market segment.

- Customer intimacy - focus on customer intimate relationships, foster a flexible and adaptive organisation as well as allow openness to new areas.

Based on these three models, the authors present a framework that provides some examples of logistics services that fit into each of these models as well as list the linked key dynamics, see Figure 4.1 below.

\begin{tabular}{|c|c|c|}
\hline $\begin{array}{c}\text { Market leadership } \\
\text { model }\end{array}$ & Examples of LSP service offerings & Key dynamic \\
\hline Operational excellence & $\begin{array}{c}\text { Pickup and delivery } \\
\text { Cross docking } \\
\text { Fulfillment } \\
\text { Basic warehousing }\end{array}$ & $\begin{array}{c}\text { Flawless } \\
\text { execution }\end{array}$ \\
\hline Product leadship & $\begin{array}{c}\text { "4PL" } \\
\text { Clean room assembly } \\
\text { Integrated information } \\
\text { Critical purchases } \\
\text { Globalisation support }\end{array}$ & Original innovation \\
\hline Customer intimacy & $\begin{array}{c}\text { Access to LSP customers } \\
\text { Joint IT development } \\
\text { Home delivery } \\
\text { "End-to-end" solutions } \\
\text { Supply chain consulting }\end{array}$ & Trust and flexibility \\
\hline
\end{tabular}

Figure 4.1 Matrix of Market Leadership Examples and Key Dynamics (source: Flowers et al., 2008, p.104).

For clarification, the authors add that an organisation may have different market disciplines for its different parts, especially when it comes to large LSPs. However, these market disciplines should be set and used at a strategic business unit level in order to support focused innovation of the LSP.

Due to the increase of new players and opportunities in the marketplace, the strategic focus and well-established strategies will most likely become an important part of LSPs' innovation success in the future. For example, those LSPs that make strategic NSD choices in relation to the external environment and internal resources needed (Zhou \& Wang, 2012), understand the role of marketing and develop creative marketing strategies for their new services (Speh, 
2008) will be those actors that achieve growth and push the development of the LSP industry further.

However, having a defined strategic focus as well as an NSD strategy may not be enough to improve efforts to develop new services. In addition to a lack of innovation-related strategies, Wagner (2008) mentions insufficient structures, process and manpower as potential reasons for the LSP industry's low innovation awareness. Hence, the relevance and existence of processes and structures adopted by LSPs when developing new services are discussed in the section below.

\subsubsection{Adoption of NSD processes and structures}

In general, there seems to be a scarcity of structured process in the literature regarding LSPs' NSD efforts. This can perhaps partly be explained by the limited research attention regarding LSPs' innovativeness as well as by a lack of empirical study regarding this in the literature. Or, as Franklin (2008) suggests, that formal processes are not employed by LSPs for reasons such as that innovation occurs as a response to a customer request and also that formal processes are viewed as too time-consuming. However, the author raises the relevant question of how, without the existence of these formal processes, LSPs aim or plan to maximise their returns from these field based innovations.

In line with the suggestions by Franklin (2008), Wagner and Franklin (2008, p.68) described the typical characteristics of the NSD process of LSPs as follows:

- "Logistics service innovation often arises not because of formal plans or processes but as an ad hoc response to a customer request"

- "Time pressures force the field team to make rapid decisions about the innovation's design in order to satisfy the customer's immediate need"

- "Innovations are usually composed of existing tools and processes combined in a novel manner, and because these tools and processes were originally developed for other applications, the solution is not generally scalable or usable for other customers with similar problems"

- "Because innovations are co-produced with the customers, the tools, processes and human factors used to create them may include components from the customer or third parties. These external components may not conform to corporate standards"

There have, however, been some attempts in the literature to describe and develop clear processes and standards that support NSD initiatives among LSPs. Three different processes with different scopes and degree of detail have been selected in order to illustrate the NSD process of LSPs. First, the logistics process innovation model by Flint et al. (2005), which can be used as a base when describing how LSPs develop sustainable logistics services and processes, see Figure 4.2 below. 


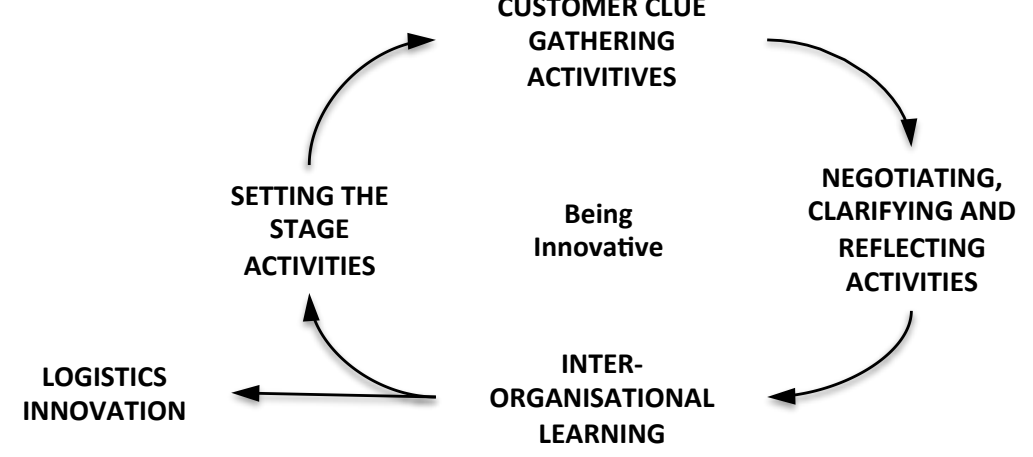

Figure 4.2 A Logistics Innovation Process Model (source: Flint et al., 2005, p.127).

The model presented is iterative in its nature and consists of four different main stages required in order to become innovative and develop new logistics services. The first stage, Setting the stage activities, as mentioned in section 4.3.1, includes activities to create an environment that allows interaction and responsiveness to customer requirements as well as being an innovative organisation. The next stage, Customer clue gathering activities, concerns activities and efforts focused on seeking and identifying information to changing and unmet customer needs. A deeper description of knowledge and skills connected to market acuity will be discussed later on in this chapter, in section 4.3.5. Moving further on in the model, the next stage, Negotiating, clarifying and reflecting activities, concerns the efforts to analyse and make use of the collected information, which is shared throughout the organisation. Lastly, the final stage in the model, Inter-organisational learning, was formulated when the authors found from their study that the participating companies had a higher focus on joint learning and innovation with customers than on organisational learning within their own company. Similar results were found in the study by Daugerthy et al. (2011), where the authors suggest that a broader base of knowledge is needed instead of only focusing on employee specialisation as an important means to generate new logistics ideas. This is also in line with Flint et al. (2008), who argue that the key to developing innovation that customers will value is how formal cross-functional and cross-organisational management of the innovation processes are handled. The authors also highlight the relevant role of supply chain and logistics managers in the innovation process, and that the more the previous processes are adopted, the greater the likelihood of good management of these innovation processes. In addition, Flint et al. (2005) argue that this responsibility is not connected to only one department, but that the responsibility and involvement should span and include multiple functions in the firm.

The second model, suggested by Busse and Wagner (2008a), is much more detailed in its design and the different stages can be compared with the new product development (NPD) processes presented in the service management literature. Using an expert panel consisting of managers from the LSP industry, the authors tried to make the innovation model more LSPspecific and break it down into features, tools needed (such as IT and management instruments) and success factors, for every single step in the model, see Figure 4.3 below. 


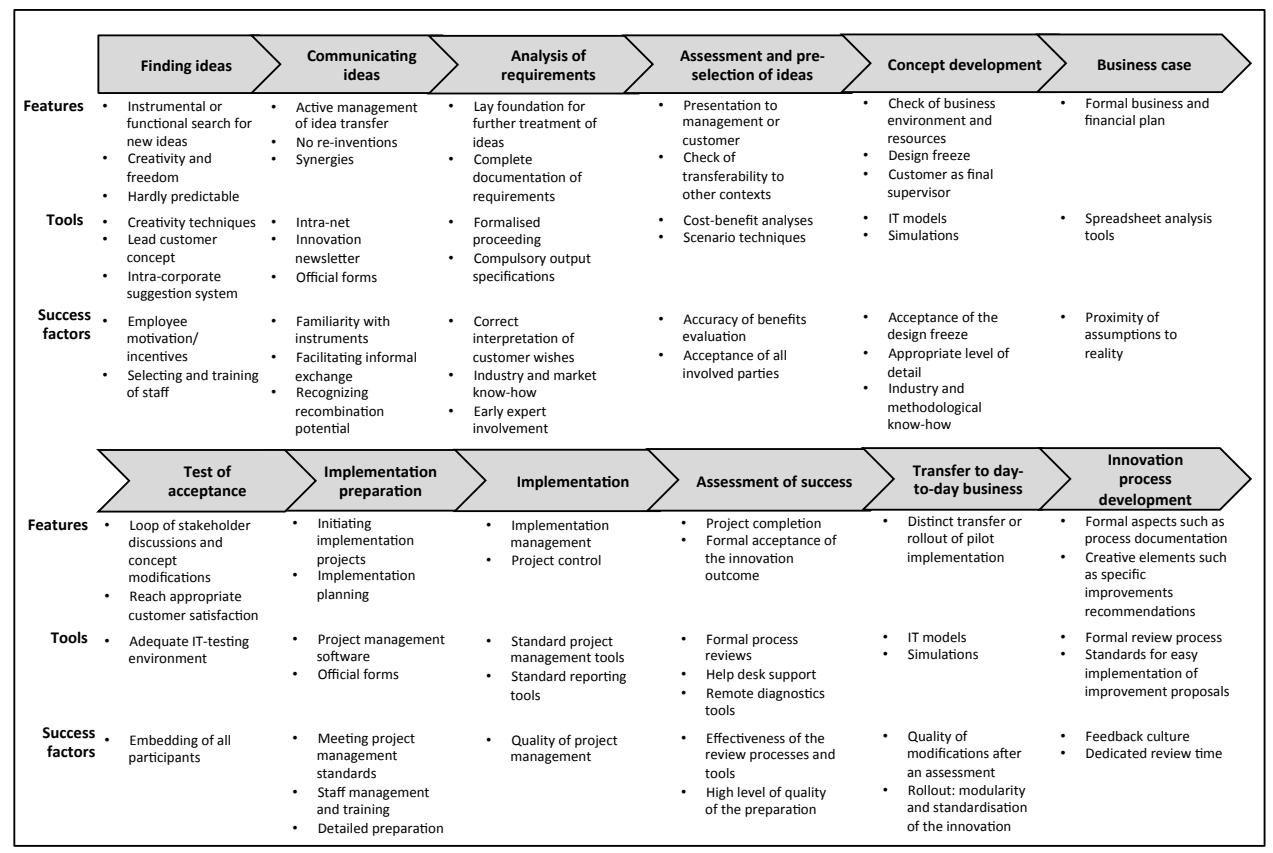

Figure 4.3 Audit Tool Model (source: Busse \& Wagner, 2008, p.131).

The model shows that both the features for the different steps, as well as the tools needed and success factors, vary throughout the NSD process, and the authors suggest that this model can be used either as inspiration or as an instrument for LSP managers to evaluate and analyse the service development process within the own company. One interesting result derived from the expert panel was that the LSP managers found the first, more creative phase of the process more challenging compared with the latter phase that involved more standardised project management. This may be because new service ideas are often proposed by customers, as mentioned earlier in this section, and not by the LSP company itself.

The third and final model of LSPs' NSD process is a "platform and toolkit" approach to LSPs' innovation management by Wagner and Franklin (2008), see Figure 4.4 below. This model has a slightly different design compared to the "step by step" approach in the models presented earlier. According to Wagner and Franklin (2008), existing structured models and processes which have been used within a range of industries to manage and control innovation have not been shown to be directly useful to LSPs. The authors argue that these models have a more product-oriented focus and do not match the distributed structure of LSPs' business models due to their rigid nature and need for central management. An innovation process focused on products is not fully appropriate for LSPs because, in order to meet unique requirements from contracting customers, it may require some kind of modification or customisation. Instead, the authors suggest that LSPs need a different kind of system that allows them to capture occurring innovations within the organisation. The system should include a certain structure and guidelines for developing new services, which will in turn facilitate for LSPs to handle customised innovations on a greater scale. 


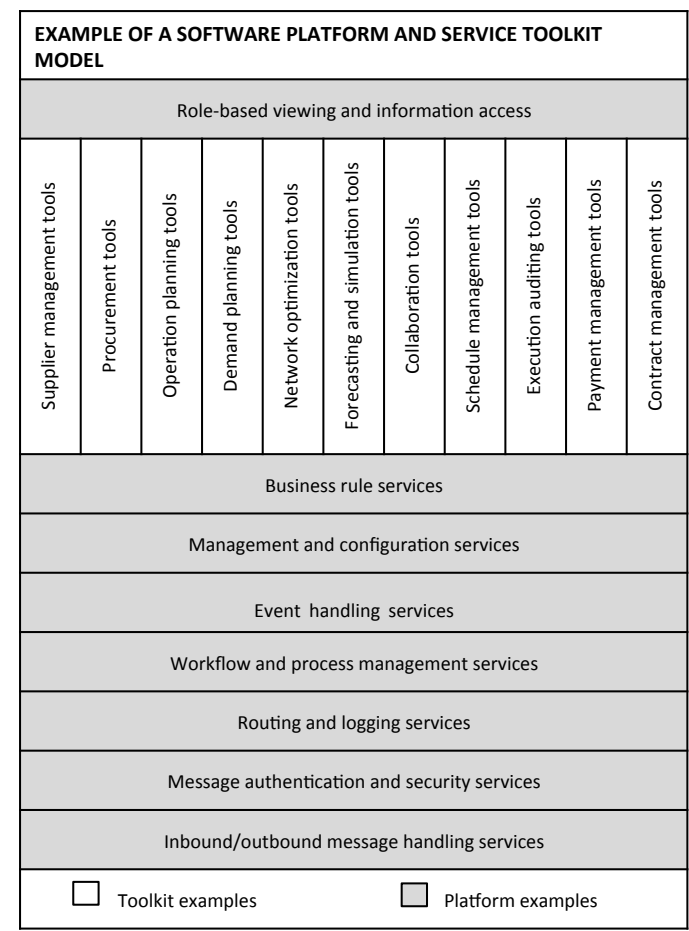

Figure 4.4 A platform and toolkit model (source: Wagner \& Franklin, 2008, p. 71).

The vertical modules suggest elements of the toolkit that should act as an aid for the employees. Each of these functional modules includes various processes, procedures, standard software application and rules in order to modify or design a service offering. The horizontal components, on the other hand, exemplify common platform services. This approach would require field personnel to focus on those innovations that can be supported by the company's IT platform as well as to use existing technology, processes and procedures that are supported within the company. The authors argue that developing specified standard "tools" would allow the rapid creation of a unique service solution to a specific customer and also a certain level of standardisation that can be reused for other customers. The authors allowed the model to be tested by an LSP and the outcome of the test run showed positive results. For example, there was a high level of customer satisfaction overall since the services were welldocumented and reliable, and the structured approach had a positive impact on service profitability due to the reduction of implementation costs and errors as well as an improvement in the service quality.

The idea of using a more "platform thinking" approach when generating and developing new logistics services can also be found in the article by Cui et al. (2009). The selected 3PLs investigated relied on their core competences (platforms) and either combined several existing service products in order to provide new offerings or added new services to the existing service products. By using their building blocks more effectively, they were able to offer customised service offerings, rather than merely providing standard service solutions. The benefits obtained from doing this were a more successful differentiation in the market, greater loyalty among existing customers and also attracting new customers. However, the authors stress that each 3PL is unique, with its own feature, which in turn depends on the type of customers and the region it serves. 
Some problems connected with the LSPs' NSD process have already been mentioned in the discussion above, for example by Wagner and Franklin (2008)'s characteristics of the LSP innovation process. Since logistics innovations seem to have been developed as an immediate response to specific customer requirements (Oke, 2008), it would be reasonable to assume that this process has been ad hoc rather than formalised. This in turn can lead to greater difficulty in managing these reactive innovations in a more formal management process (Oke, 2008) as well as in replicating successful innovations (Flint et al., 2005). Oke (2008) mentions other barriers connected with the innovation process, such as the difficulty of testing the new concept, since this may require a closer relationship with the customers. The amount of potential test companies may be limited depending on how many customers the LSP has a close relationship with. Another barrier mentioned by Oke (2008) concerns the high focus on technical innovation. In order to make this happen, LSPs may become dependent on external partners and thus integrate their own processes with the partnering companies' processes, which can be a challenge for LSPs, especially if they lack effective development processes.

However, what is needed in order to succeed with the structure and management of NSD process among LSPs? In the study by Busse and Wagner (2008a), different success factors connected to the innovation process were highlighted, such as obligatory structuring of flow and decision-making as well as integrity of the processes. In general, it appears to be vital to develop some kind of process or structure in order to manage NSD within LSPs. In addition, the study by Daugherty et al. (2011) indicates that more formalised procedures support rather than prevent innovation among LSPs. The results revealed a positive relationship between formalisation of processes and the degree of logistics service innovation. Even if a high degree of formalisation has been suggested as being harmful to NSD efforts, the authors argue that formalisation of required activities or procedures will not necessarily inhibit new idea generation. This is in line with Franklin (2008), who states that without any mechanisms (such as formal processes and procedures) for channelling the new service development activities that occur with company, it can be hard for LSPs to gain the full benefit of the different innovations developed within the company.

In addition, Busse and Wagner (2008a) stress the need for LSPs to modify existing innovation concepts and instruments in order to create more industry-specific approaches that fit the characteristics of LSPs. The authors mentioned LSP characteristics such as "down-to-earth character", a relatively low level of educated employees, as well as an often decentralised organisational structure. Oke (2008), on the other hand, stresses the need for a flexible structure and argues that LSPs should also create different processes for different types of innovation. Furthermore, the author stresses that the existence of an innovation process confirms both accountability and consistency in terms of what is provided to their customers. Pekkarinen and Ulkuniemi (2008) also stress the importance of standardisation of services and processes for LSPs and mention benefits such as efficiency improvements and segmentation of both service offerings and customer relationships. In addition, it is relevant for LSPs to increase their ability to capture repeating benefits from the service development efforts. Chapman et al. (2003) suggest that LSPs must take a broader perspective and consider the full extent and breadth of their function, systems and processes throughout the supply chain. Focus should also be put on coordination of activities among the partners in the network in order to gain new knowledge and integrate it into the service development process.

The next section will highlight in more depth some aspects which have been mentioned in the previous section, namely the role of IT integration and information sharing when developing new services among LSPs. 


\subsubsection{The multifaceted role of information technology}

The rapid development of information and communication technologies has for some time been identified as a significant driving force for the growth of many service firms as well as a source of influence for innovation efforts (Chapman et al., 2003). The transport and logistics industry is no exception. The evolving ICT trend has resulted in more complex logistics service offerings and customers' requirements have become more information-intensive and moved far beyond traditional transportation needs. Therefore, the relevance and application of new technology has come to play an important role in LSPs' service development efforts (Zhou \& Wang, 2012) since many new service initiatives among LSPs are based on or rely on technological developments and innovations (Oke, 2008). In addition, IT capabilities have come to be viewed as an essential part of an LSP's ability to add extra value to its customers and other stakeholders in the network (Chapman et al., 2003) as well as a key aspect and opportunity to drive innovation (Third-Party Logistics Study, 2013).

Due to the transportation and logistics industry's need for information in order to improve the efficiency of their operations, the adoption and integration of ICT systems can result in a major competitive advantage for these companies (Chapman et al., 2003). Or, as Oke (2008) expresses it, due to the high market competition and customers' increasing demands regarding more technological competences and IT solutions, "LSPs can not afford not to adopt leadingedge transportation and warehousing management systems" if they want to maintain market standing and continue to improve their service offerings (p.19).

Adoption of various technologies, in particular IT innovations, has come to play an important role in ensuring the flexibility and efficiency of the whole system and it also constitutes a valuable part in LSPs' development of new services (Pekkarinen \& Ulkuniemi, 2008). Growing numbers of LSPs have started to adopt advanced information systems, real-time tracking systems, automatic storage, loading and unloading equipment, which in turn has led not only to an increasing number of new services but also to the improved efficiency of some services (Zhou \& Wang, 2012). Thus, adoption of various technologies can be seen as a great opportunity for LSPs to develop and offer new services that reach beyond the company's core business concept. This is confirmed by the results of the study by Lin (2008), which reveal that many of the LSPs studied started to adopt innovative logistics technologies in order to enhance their services. In addition, an improvement of supply chain performance would also occur for those LSPs that had a favourable attitude and showed willingness towards the adoption of innovative logistics technologies. According to the author, logistics technologies can include various aspects such as data acquisition technologies, information technologies, warehousing technologies and transportation technologies. By adopting these kinds of technology innovations, LSPs can provide customers with increased visibility and tracking of movements of orders, shipments and inventories as well as co-ordination and integration of activities (Oke, 2008).

As mentioned earlier, adoption of ICT creates opportunities for LSPs to enhance existing service offerings and also to develop new kinds of offerings. However, ICT also provides a basis for knowledge sharing within and among the organisations involved (Chapman et al., 2003) and it allows incorporation of new functions as well as commitment to new alliances (Evangelista \& Sweeney, 2006). In addition, since it will facilitate organisational communication this may also have a positive impact on the creation of logistics innovation capability (Daugherty et al., 2011) as well as reaping benefits from their innovative efforts (Chapman et al., 2003). Other mentioned benefits that IT leads to are enhanced productivity, cost reductions, the provision of innovative and customised services and improved service 
quality (Wagner \& Sutter, 2012). However, to fully achieve these benefits there is a need for synchronisation of these new technologies (especially ICT) in order to have a more streamlined management process and to achieve improvements in efficiency and productivity across the supply chain (Chapman et al., 2003).

The annual study of Third-Party Logistics (2013) revealed that even if growing numbers of LSPs have started to adopt new and advanced technology, the customers rate LSPs' IT capabilities as rather low and describe their IT investments as mainstream and conservative, a difference that has become known as the IT Gap. Figure 4.5 below shows the difference between LSPs' customer satisfaction with basic IT services and LSPs' ratings of their own IT capabilities.

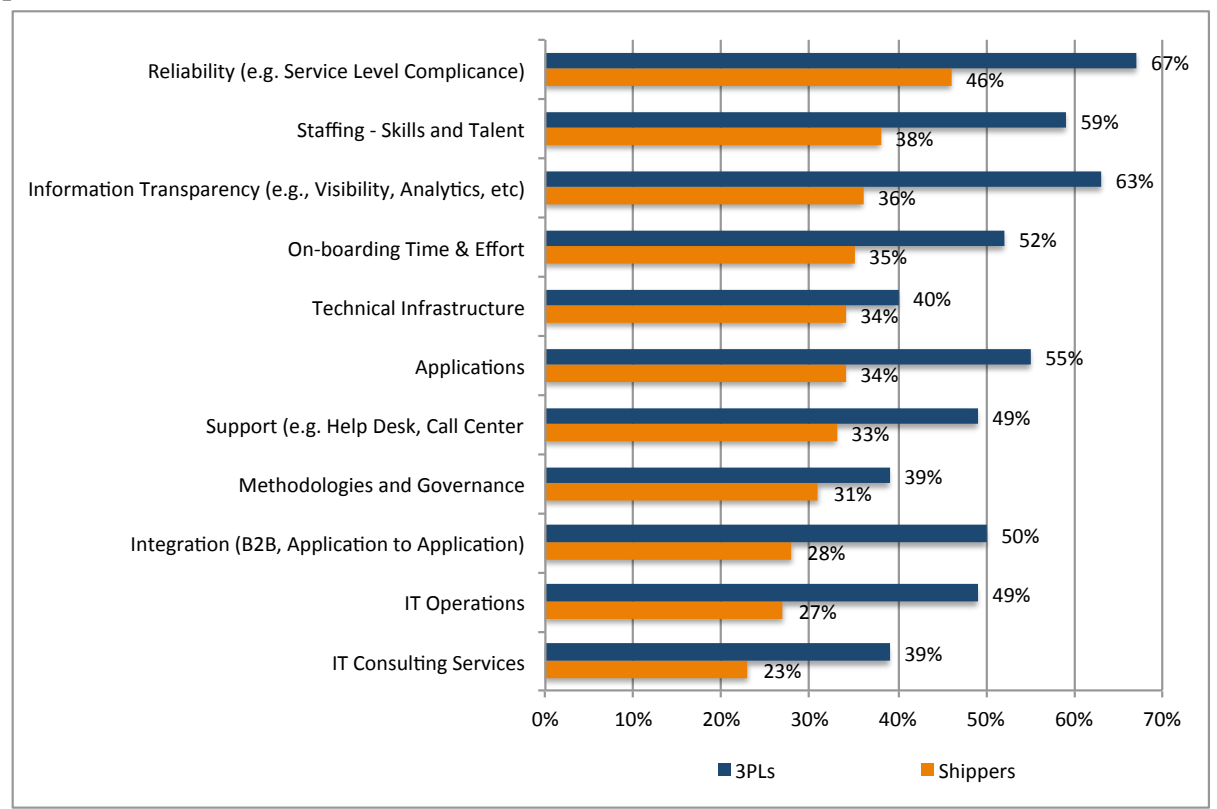

Figure 4.5 Perceptions of 3PLs IT capabilities (source: Third-Party Logistics Study 2013, p. 21 ).

As can be seen in the diagram, there seems to be some mismatch between customer satisfaction and LSPs' own perceptions of their IT capabilities. However, as suggested in the study, this can be seen as an input to opportunities for LSPs to improve their IT capabilities as well as to improve their technical relationships with their customers. In addition, the study also reveals that $55 \%$ of the shippers studied desired a more strategic technical relationship with their 3PLs. When it comes to the 3PLs, half of the respondents state that they are planning to make larger IT investments in the future. However, it is also stated in the study that these investments must be made based on what their customers need, which in turn requires a good understanding of their customers' supply chains and their challenges. Moreover, by working with customers on different IT solutions, LSPs will gain valuable technology-specific knowledge as well as expertise within this area (Wagner \& Sutter, 2012).

However, even if technology can be an enabler of innovation for LSPs, it can also be a major barrier. Wagner and Sutter (2012) mention that due to rapid changes in technology, increased customer demands for system customisation and an unwillingness to pay for the cost of these applications leaves LSPs with high costs and low returns on IT investments. Oke (2008) 
mentions another issue: since technical innovations may not be the core of LSPs, partnerships with technology companies are perhaps initiated in order to develop specific technology for the use of the LSP. According to the author, this can put LSPs in a difficult and powerless situation since these innovations may not be owned by the LSP itself and thus create a huge dependence on the company developing these kinds of technologies. The more critical the technical innovation is for the LSP, the greater is the risk of dependence on the partnership company. In addition, Oke (2008) states that even if technology can be an opportunity of innovations it can also limit the amount of innovation that LSPs can engage in, since it is not their core competence.

To sum up, the previous section has revealed that technology not only constitutes an important component of logistics service offerings but can also facilitate information sharing within the LSP's own company as well as with other parties involved in the company's network. Therefore, technology can be seen as an enabler to make creative use of the knowledge and relationships network, which in turn can lead to rapid service development capabilities (Chapman et al., 2003). However, the negative side-effects of the technology development, which in turn lead to a need for LSPs to increase their knowledge and skills regarding IT, both in general but also connected to their service development efforts, were also highlighted. The next section aims to explore other knowledge and skills needed regarding LSPs' service development efforts.

\subsubsection{Knowledge and skills needed for LSPs' NSD}

In section 4.3.1, the relevant roles of employees, cross-functional teams as well as collaboration with external partners such as customers and suppliers when developing new services were discussed. This section aims to take these aspects one step further, as well as look at the ability of LSPs to find customer and market information and how this is used in the development of logistics services.

To take LSPs' knowledge and skills regarding market orientation first, many studies show its importance for their overall performance (Ellinger et al., 2008) as well as for a possible contribution to development of service offerings (Fugate et al., 2008). In order to create a more market-oriented company and discover new business opportunities, Chapman et al. (2003) stress the need for LSPs to frequently search for new knowledge, keeping the customers in mind when developing new services. However, even if it is important for LSPs to be more proactive and innovative, this does not happen very often, and the outcome tends to not quite match the customer' expectation (Busse \& Wallenburg, 2011). Thus, Rajesh et al. (2011) suggest that LSPs should tailor their capabilities in order to meet customers' increasing needs and expectations, since the prior research has shown that customers' expectations of logistics services are higher than the services actually received.

Therefore, insights from customers can be seen as an essential source for idea generation for LSPs, which in turn requires a certain ability on the part of LSPs to scan off the market and look for customer information as well as to integrate this information into the service development process. Input to LSPs' idea generation may arise from different sources, such as direct customer interaction, monitoring the market environment, report of appraisals or complaints as well as from analyses regarding customer buying and secondary data (Flint et $a l ., 2008)$. To create a more strategic and effective approach to capturing, understanding and identifying trends and common themes of field-base knowledge, Wagner and Franklin (2008) suggest that this will require LSPs to adopt different kinds of sensing and analysing tools, such as project debriefings and online project archives. This argument is supported by Flint $e t$ 
al. (2005), who found in their study that the procedure regarding market orientation was informal rather than formal, and stressed at the same time a need to create systems for collection, analysis, integration and dissemination of different kinds of data. In addition, the authors also present a summary of the representative examples of customer clue gathering activities found in their study, see Figure 4.6 below.

\begin{tabular}{|c|c|}
\hline Activity & What gets done \\
\hline \multicolumn{2}{|l|}{ Direct means } \\
\hline Customer groups & $\begin{array}{l}\text { Customers present to LSP } \\
\text { Customers listen to LSP } \\
\text { Customers serve on a panel in front of LSP }\end{array}$ \\
\hline Formal depth interviews & $\begin{array}{l}\text { One LSP manager conducts one-on-one, open-ended, discovery interview while } \\
\text { another LSP manager takes notes }\end{array}$ \\
\hline Extended customer retreats & $\begin{array}{l}\text { Spend several days off site exploring strategic objectives and processes in a very } \\
\text { informal setting }\end{array}$ \\
\hline Joint strategy meetings & Formal meetings designed to align customer and LSP strategic plans \\
\hline Draw on outside expertise & $\begin{array}{l}\text { Tap into academic and consultant expertise to conduct qualitative and quantitative } \\
\text { research }\end{array}$ \\
\hline \multicolumn{2}{|l|}{ Indirect means } \\
\hline Reading trade journals & Search for clues to market change \\
\hline $\begin{array}{l}\text { Attending industry } \\
\text { conferences }\end{array}$ & Discuss potential changes with others in industry \\
\hline Analyse customer data files & Look for shifts in usage, needs, opportunities \\
\hline Analyse performance metrics & Look for customer-focused innovation opportunities \\
\hline $\begin{array}{l}\text { Monitor technological } \\
\text { changes }\end{array}$ & $\begin{array}{l}\text { Contemplate how technological change will impact customers' business or LSP's } \\
\text { ability to provide added value to customers }\end{array}$ \\
\hline Monitor competitor changes & $\begin{array}{l}\text { Contemplate extent to which new competitor offering better serves a customer need } \\
\text { and suggests a change in what customers value }\end{array}$ \\
\hline Overall compiling & $\begin{array}{l}\text { Pull together all customer clues into a holistic insight to potential changes in what } \\
\text { customers value and innovation opportunities }\end{array}$ \\
\hline
\end{tabular}

Figure 4.6 Examples of customer clue gathering activities (source: Flint et al., 2005, p. 133).

The authors noticed that the activities found in the study have a strong focus on gaining customer insights. However, there was less focus on the development of technology, competitors or identifying different trends or scenarios on the market. Despite the general perception that customer insights and understanding are necessary and a valuable input when developing new services, the study by Flint et al. (2008) revealed that LSPs were one of the industries that scored the lowest on monitoring the market environment. In addition, even if customers are seen as an important source of ideas for LSPs (Busse \& Wallenburg, 2011), there exists a belief that LSPs should be more proactive and develop new services not solely based on the voice of the customers (Flint et al., 2005). In addition, Gammelgaard (2008) suggests different potential pitfalls regarding logistics innovation and mentions a general lack of openness towards the environment as one of these. Hence, identifying different trends, issues and market changes should not be neglected, since this may to some extent explain the current demands from customers and how these may develop in the near future. This is supported by Flint et al. (2008) who state that LSPs need to follow changes in the economic and regulatory environment, technological innovations as well as mergers and acquisitions within the supply chain. Furthermore, the authors highlight the relevance of combining the expertise of supply chain managers and logistics managers with the expertise of markets or sales professionals for activities connected to customer and market orientation. This is in line 
with the study by Panayides (2007), the results of which support earlier findings that crossfunctional information sharing within the company improves logistics service quality.

In order to make the best use of the collected information and generate ideas that will eventually lead to the introduction of new services and solutions on the market, Flint et al. (2005) stress that LSPs might evaluate the level of employees' competences regarding these issues. In general, skilled and trained employees have come to play a more important part for LSPs in their efforts to develop and provide more value-added services (Jumadi \& Zailani, 2011). In addition, the authors stress that there is a greater need for multi-skilled generalist than technically-oriented competencies in the effort to succeed in the competitive and complex business environment of LSP. Furthermore, creating a more customer-centric culture places requirements on the employees to "think outside the box" regarding how customers' requirements are changing and then improving services and systems in order to meet these changes (Flint et al., 2005). However, to succeed with this approach, the authors argue that there is a need to train employees in this kind of thinking. An example of the valuable managerial role of coaching front-line service employees can be found in the study by Ellinger et al. (2008). This kind of support was shown to have a positive effect on both market orientation performance and employee and organisational performance. However, service-related training was only shown to improve employee performance, not organisational performance. A potential explanation for this according to the authors is the fact that market orientation is rooted in an organisation's culture and is not easy to change in the short run. Furthermore, the authors stress that the way LSP managers choose to support their market orientation activities with initiatives such as employee development practice is specific to each company due to its unique culture, structures and environments.

In addition to the focus on gaining more knowledge form external partners, LSPs are also advised to create and develop internal processes and capabilities in order to identify external sources of innovation as well as potential collaboration partners when developing new services (Wagner, 2013). In his study (2013), Wagner investigates the extent to which LSPs utilise external knowledge as well as which collaboration partners they choose in their innovation efforts. In general, the results show that the use of external sources as an input to innovation was not used to any great extent. Despite the low level of utilisation, customers, suppliers and competitors are seen as an important source of innovation and the author suggests LSPs should intensify their relationships with these actors. However, Flint et al. (2008) state that few LSPs engage in logistics innovation with their customers. This can perhaps be partly explained by Gammelgaard (2008), who suggests potential difficulties connected with customer collaboration such as "lack of long-term dedication, lack of information sharing and mutual openness, lack of support connected to the innovation from the customer organisation" (p.143).

However, the result of the study by Wagner (2013) shows that utilisation of external sources can indeed have a valuable role and input in the improvement and/or development of new logistics services. For example, customers, suppliers and competitors were all positively related as a source to service improvement while only customers were positively related to service new to the firm. In the study, consultancies and universities were also investigated as a potential external source of innovation, but these actors were singled out as having a positive impact on service improvements and development of new services. However, the authors stress the indirect relevance of the actors for LSPs' efforts to develop new services. These actors can have a more supportive function in LSPs' further NSD efforts in the form of an input to the development of strategies, process and structures connected to the 
development of new services. In addition, the author proposes that the development of the ability to use and integrate external partners in the process of developing new services can potentially be a competitive advantage that distinguishes one LSP from another. Furthermore, the study by Bellingkrodt and Wallenburg (2013) shows that the relevance of using and integrating external sources may depend on the specific focus and aim LSPs may have on their innovation efforts. For example, those LSPs that strive for improvements of internal processes or for improved services for current customers, benefit from improved relationships with these customers, while those LSPs that strive to reach new customers and business opportunities benefit from improved relationships with external service providers and horizontally cooperating LSPs.

Integrating more closely with both customers and suppliers can lead to a more focused and positive outcome of the service development efforts. Flint et al. (2008) give some examples of what this may involve, including "product modifications, entirely new products, supply chain modifications such as network redesign, transportation route and modelling, package design, information systems modifications or enhancing goods in transit visibility through the application of emergent technologies" (p. 274). However, in the reviewed literature regarding integration of external partners when LSP develop new services, the main focus is on customer integration. The integration and role of suppliers and other external actors in a service development context of LSPs has received very little attention in the reviewed literature. One possible explanation for this is that customers have been highlighted as playing an important role in LSPs' NSD and therefore much attention has been given to these actors in research. For example, Wagner and Sutter (2012) show in their study that joint innovation efforts between LSPs and customers is influenced by factors such as high integration with customers, establishing links to customers insisting on new services, complementary relationship-specific investments and agreement on benefit sharing. In addition, the authors argue that integrating in such joint innovation efforts strengthens both the position of LSPs as well as customer relationships and also leads to enhanced performance. Flint et al. (2008) propose that managers of LSPs might consider additional ways of integrating more with their customers as well as how they should be involved, and at what point in the service development process.

One example of different approaches to customer integration can be shown in the study by da Mota Pedrosa (2012), which revealed that both reactive and proactive customer integration are valuable as well as necessary in successfully anticipating customers' current and latent needs during the development process of new services. A proactive customer integration approach concerns integration with customers into workshops in order to evaluate, prioritise and develop innovative ideas. This approach led to LSP advantages such as deeper and more detailed understanding of customers' requirements, as well as of business and environments that can be used in order to develop more customised service offerings to the customer. A reactive customer integration approach, on the other hand, involves day-to-day interactions that help LSPs obtain access to the current customer problems and also to co-create knowledge. The result revealed that different approaches to customer integration are important to stimulate and initialise co-creation of knowledge, which in turn have a positive effect on LSPs' NSD capabilities. In addition, the results demonstrate the relevance of planning and deciding what kind of role customers are going to play in the NSD process in order to find a balance between cost and outcome based on the NSD efforts. When, and to what extent, customer integration is adopted can also depend on what kind of service is developed. Further, da Mota Pedrosa (2012) also stresses that not being solely customerdriven and basing service development on reactive customer integration, provides LSPs with 
the space and opportunities to develop new services based on their own strategy and value beliefs.

\subsection{Towards a framework for LSPs' management of NSD}

Each of the dimensions discussed above has its own purpose and goes some way to providing an understanding of LSPs' NSD, but by combining these dimensions and linking them to each other, a more complete understanding regarding their ability to develop new services can be reached. In order to ensure that the service development efforts are successful, LSPs should consider innovation processes as a system, and progressively manage the innovation system with effective and proactive strategic decision (Shen et al., 2009). This is in line with the reasoning of Busse and Wallenburg (2011), who highlight the need for a more holistic view of innovation management among LSPs, while stressing that this view should involve both a systematic and a procedural perspective. In addition, Busse and Wagner (2008a) also stress the need to extend the current process-oriented view to a systems perspective of LSPs' innovation management. A company's NSD should perhaps not be viewed as a single function; rather, Li et al. (2005) describe it as a network, where each stage is driven by the feedbacks between the different stages. However, by viewing NSD as a network, the authors stress the need for a system perspective, with guidelines set at the firm level as well as transparency regarding the frames and connections among the NSD elements.

As mentioned earlier in this chapter, LSPs are advised to develop and design concepts of NSD management systems, as well as processes. However, this design must be formed in such a way that it matches the company's organisational and environmental circumstances (Busse \& Wallenburg, 2011). Another relevant aspect regarding the NSD management systems concerns its degree of flexibility. For example, Zhou and Wang (2012), stress that the key links of NSD (such as strategy analysis, project team organisation, technological choices, market testing and so on) may vary due to the varieties of logistics services. As mentioned earlier, the NSD management system should not only fit the company's internal organisational environmental but also allow an external focus on the company's surrounding environment. By taking a more holistic perspective of different processes and systems in the supply chain, as well as striving to synchronise activities among involved partners, LSPs can improve their innovation capabilities and gain valuable knowledge as an input to their innovations efforts (Chapman et al., 2003). However, due to the involvement of several parties in the supply chain, logistics innovation is often hard to manage (Gammelgaard, 2008).

As stated earlier in this chapter, no best practice regarding NSD among LSPs seems to exist in the literature. However, as a first step towards increasing the empirical understanding and gaining insight into a specific LSP's NSD management, the conceptual framework of NSD management (presented in Chapter 3) is used as a base, see Figure 4.7 below. The framework is used in order to structure and identify an LSP's awareness and status of each of the included dimensions (NSD culture, NSD strategy, NSD process focus, IT use and expertise and NSD knowledge and skills).

Further, each dimension has resulted in some survey questions, which have been used as a guide in this dissertation for the empirical investigation of a selected LSP's NSD management. The motivation of each survey question can be found in the discussion below. 
First, the development and existence of NSD culture within an LSP. This aspect is perhaps the most challenging for LSPs to create, but development of a culture that promotes and fosters NSD is also highlighted in the literature as playing an essential role in a company's ability to develop new services. This dimension is all about creating and managing an environment that values NSD and allows learning internally within the organisation and between different functions, as well as externally with customers and other parties. So, the first survey question concerns the LSP's awareness of a NSD culture and which aspects this may involve:

- What characterises a culture that fosters NSD within an LSP?

The second dimension concerns the strategic focus and existence of an NSD strategy within an LSP. As discussed in section 4.3.2, the strategic focus or NSD strategy can be regarded as the hub that combines the various NSD activities. In addition, to some degree it provides the direction and identifies the focus areas for a company's NSD efforts. However, since NSD or innovation can involve different views in a company, it would therefore be relevant to identify which aspects an LSP may involve in its strategic focus or NSD strategy within its own company:

- Which aspects are involved in the strategic focus of an LSP's NSD efforts?

The third dimension concerns the NSD process focus. The literature advises LSPs to create more formalised structures and processes in order to facilitate management as well as recap lessons learned of its NSD efforts for a specific customer to a larger customer base. However, the NSD process among LSPs is often described as an ad-hoc response to a specific customer. Hence, it may be relevant to obtain an insight into how an LSP structures its NSD process in order to fit with the company's strategic NSD focus as well as to facilitate scalable solutions for other customers with similar requests:

- How does an LSP structure the process from idea generation to the launch of a new service?

The fourth dimension concerns the IT use and expertise connected to NSD efforts. The evolving ICT trend has affected the LSP industry in many ways, not least from a service development perspective, and has resulted in more complex and informative logistics service offerings. Adoption of various technologies has become a valuable part of LSPs' NSD, and in order to obtain a deeper insight into an LSP's awareness of its relevance connected to service development, the following survey question was formulated;

- What impact does IT have in an LSP's NSD efforts?

The last dimension concerns knowledge and skills connected to NSD. Section 4.3.5 discussed aspects such as an LSP's ability to find market information as well as use in-house knowledge as an input to its NSD efforts. This dimension also involved the existence of collaboration with external parties in order to integrate external knowledge within the NSD process. So the final survey question is:

- Which internal and external knowledge is used as an input and how is this knowledge integrated in an LSP's NSD process? 


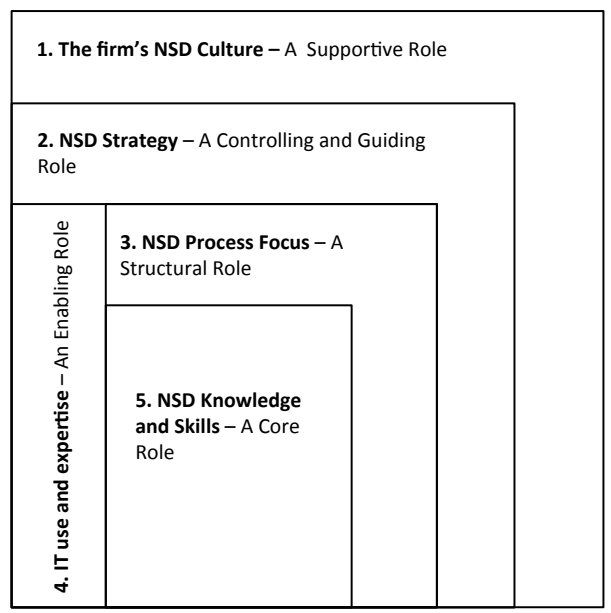

1. What characterises a culture that fosters NSD within an LSP?

2. Which aspects are involved in the strategic focus of an LSP's NSD efforts?

3. How does an LSP structure the process from idea generation to the launch of a new service?

4. What impact does IT have in an LSP's NSD efforts?

5. Which internal and external knowledge is used as an input and how is this knowledge integrated in an LSP's NSD process?

Figure 4.7 The empirical guide for an LSP's management of NSD.

To conclude, the conceptual NSD framework together with its including dimensions, developed in Chapter 3, appears to be applicable on LSPs for describing and structuring their NSD efforts. The content in the different dimensions differs to some extent since these dimensions are only partly covered in the literature, challenges as well as aspects relevant to take into consideration in NSD efforts particularly among LSPs.

In addition, as mentioned before, the survey questions are used as means to structure the empirical data, and the complete empirical description of the selected LSP in this dissertation can be found in Chapter 6 . Next, Chapter 5 will give insight into an area that has come to affect and put pressure on LSPs' operations as well as on their service offerings, namely environmental and climate change. 
$-78-$ 


\section{CHAPTER}

\section{THE GREEN DEVELOPMENT AMONG LSPS}

This chapter aims to give an insight into how the discussion and increased environmental awareness regarding sustainability have affected the LSP industry and its service development. Both potential drivers as well as barriers affecting the willingness of LSPS to develop green services are mentioned. Further, the chapter aims to identify and give an understanding of aspects that may influence and facilitate the development of green services among LSPs. In addition to prior research from other researchers, this chapter includes results from the researcher's Licentiate thesis as well as additional papers. Empirical data derived from the questionnaire survey presented in the Licentiate thesis can also be found in this chapter. This empirical data has not been previously presented or published. The chapter ends with a summarisation resulting in some survey questions, as in Chapter 4, in order to guide the further empirical investigation regarding LSPs' environmental work and development of green service offerings. 


\subsection{Green logistics - a new area of NSD among LSPs}

Increased pressure from society, as well as requirements from customers, has forced companies to become more aware of their impact on the environment. Sustainability awareness has become an essential part of a business's operations and requires an active management (Prockl \& Gammelgaard, 2012). The environmental pressure has led to a transformation of companies not only in terms of their business models but also in the range of service solutions provided. This is also true for the transportation and logistics industry. Due to increased requirements from governments and customers, LSPs need to address more efforts regarding environmental issues (Wolf \& Seuring, 2010) and start acting to reduce the impact on the environment (Gammelgaard, 2008) in order to provide green service offerings to customers. Developing green services by integrating environmental management into service development efforts is therefore believed to be a promising and important area for the LSP industry (Ho et al., 2009).

It has been suggested that the LSP industry should focus more actively on environmental issues and act as the "manager of sustainability within the supply chains" (Prockl \& Gammelgaard, 2012). Many LSPs have realised their impact on the environment and started to take responsibility for their actions. For example Mena et al. (2007) mention actions undertaken by the LSP industry such as improved forward and reverse chains integration in order to achieve vehicle utilisation improvements and emissions reductions.

Other initiatives undertaken by LSPs in order to reduce the environmental burden are shown in Table 7 and Table 8 below.

Table 7 Transport related measures adopted by LSP to reduce the environmental impact.

\begin{tabular}{lccc}
\hline Transport related measures & N & Mean & Std. Dev. \\
\hline $\begin{array}{l}\text { Transport planning management (e.g. route } \\
\text { optimisation) }\end{array}$ & 58 & 4.00 & 1.23 \\
Eco-driving & 58 & 3.95 & 1.34 \\
Offer documented emissions data & 56 & 3.59 & 1.47 \\
Measures to reduce empty running & 57 & 3.54 & 1.36 \\
Measures to improve vehicle loading & 57 & 3.37 & 1.35 \\
Modifications to vehicle specifications/design (e.g. & 57 & 2.91 & 1.50 \\
Aerodynamic features, low rolling resistance tires) & & & \\
$\begin{array}{l}\text { Structural changes to your logistics system (e.g. } \\
\text { number, size or location of facilities) }\end{array}$ & 53 & 2.83 & 1.45 \\
$\begin{array}{l}\text { Alternative fuels for transports } \\
\text { Greater use of intermodal transport measures to }\end{array}$ & 56 & 2.79 & 1.37 \\
reduce empty running & 56 & 2.57 & 1.69 \\
Recurrent environmental pilot project & 56 & 2.54 & 1.38 \\
Switch to less energy-intensive transports modes & 57 & 2.40 & 1.47 \\
\hline
\end{tabular}

As can be seen in Table 7, the measures that received the highest means were transport planning management, eco-driving, offer documented emissions data as well as measures to reduce empty running. These measures were also significantly higher than all the other measures. Less commonly adopted measures included alternative fuels for transports and switching to less energy-intensive transport modes. This can perhaps partly be explained by Isaksson (2012), who points to the economic barriers when investing in environmentally- 
friendly vehicles and also the issue regarding limited gas stations for alternative fuels. When it comes to supply chain-related measures, environmental certification received the highest mean and it was also significantly higher than all the other measures, see Table 8. Setting a corporate GHG emission reduction target was also a common measure among the LSPs studied and was significantly higher than most other measures (with the exception of environmental certification and environmental education for employees). In addition, it also seems that in the effort to meet environmental targets, cooperation with suppliers/partners is more common than cooperation with customers. The cooperation with suppliers measure was also significantly higher than cooperation with customers.

\begin{tabular}{|c|c|c|c|}
\hline Supply chain related measures & N & Mean & Std. Dev. \\
\hline Environmental certification (e.g. ISO 14001) & 59 & 4.00 & 1.54 \\
\hline Setting a corporate GHG emission reduction target & 56 & 3.70 & 1.41 \\
\hline Environmental education/training/information for employees & 59 & 3.59 & 1.30 \\
\hline Increase the amount of waste recycled & 56 & 3.34 & 1.35 \\
\hline $\begin{array}{l}\text { Cooperation with our suppliers/partners in order to reach } \\
\text { environmental targets }\end{array}$ & 59 & 3.27 & 1.38 \\
\hline $\begin{array}{l}\text { Cooperation with customers in order to reach environmental } \\
\text { targets }\end{array}$ & 60 & 2.93 & 1.35 \\
\hline Environmental Management System (EMS) & 55 & 2.76 & 1.59 \\
\hline Collecting information on energy use/carbon footprint & 56 & 2.55 & 1.45 \\
\hline Renewable/green energy & 57 & 2.33 & 1.53 \\
\hline Reduction in the amount of packaging & 54 & 2.19 & 1.26 \\
\hline $\begin{array}{l}\text { Environmental education for our customers and/or suppliers } \\
\text { partners }\end{array}$ & 59 & 2.00 & 1.20 \\
\hline Emission off-set programmes & 55 & 1.67 & 1.04 \\
\hline
\end{tabular}

The majority of the most commonly adopted measures have a more internal than external focus. This in turn suggests that LSPs have first started to improve the environmental impact of their activities internally. The focus has therefore not concerned a more external focus, for example how to collaborate with customers, offer environmental education or develop green services available for the market. However, one limited factor in the development of this approach may depend on the attitude and interest shown towards green concerns on the part of the customers. The next section presents and discusses various barriers and drivers that may have an impact on further green development of LSPs. These factors are explored in order to gain a deeper understanding of how organisational and environmental factors can affect the development of green service offerings.

\subsubsection{Drivers and barriers towards adoption of green initiatives}

Even if growing numbers of LSPs have started to adopt green initiatives in order to reduce the environmental burden of their business activities, the green development within this industry is still seen to be in an early stage of its full potential. Due to its infancy phase, Lin \& Ho (2008) suggest that the efforts to develop green services adoption can be viewed as an innovative process for LSPs. This is in line with Gammelgaard et al. (2012), who state that developing green logistics services does not differ significantly from developing other kinds of logistics services and can therefore be regarded as logistics service innovation. The way to view development of green logistics services from an innovation perspective is also adopted in the article by Isaksson and Huge-Brodin, (2013). The purpose of the article was to indicate the status of LSPs and their underlying rationale in the development of green service 
offerings. Based on literature describing different dimensions characterising a service innovation and empirical input from six different LSPs, the authors could discern three different patterns, namely:

- Focus and abide versus network and explore. This pattern concerns the strategic standpoint of the greening of LSPs' activities, such as either offering customers a green choice or option, or treating green aspects as fully integrated in the general service offering. Furthermore, this pattern also reveals the LSPs' competitive awareness of green development in the logistics market.

- Internal focus for general "spread" versus broad engagement to target specific customers. In general, this pattern focuses on customer orientation and the transfer and sharing of green knowledge and competences. More specifically, the LSPs in the first group address green offerings to all, or a wide range of, their customers and organised their green efforts internally through specialised departments. In the second group, green offerings were addressed to specific customer segments and the environmental competence was broadly spread through the organisation.

- Top-controlled passiveness versus bottom-up exploration of technology. The last pattern involves the management and control of green initiatives, as well as the attitude towards technology investments. In the first group, there was a clearer focus and support from top management regarding green initiatives. However, at the same time, these LSPs showed a more restrained attitude towards introducing new technologies that support greening of their logistics activities. In the second group, there was thus a stronger focus on bottom-up management of green aspects, and these LSPs showed a greater desire to find new technologies in order to support greening of their activities.

Based on the result from the study, the authors reveal a broad picture of the current status and strategies adopted to address green development of LSPs and their service offerings. However, the authors argue the differences found can partly be explained by the differing nature of the LSPs studied but also due to the fact that the development of green logistics service seems to be in an early phase.

Some drivers and barriers regarding LSPs' adoption of green initiatives and attitudes towards developing green services are presented below. The identification of these factors can be seen as a first step in understanding how LSPs can manage the development of green services.

The answer to the question of why LSPs start to adopt green initiatives within their companies is that there appears to be a desire to do the right thing, both regarding environmental concerns as well as meeting customers' demands (Lieb \& Lieb, 2010). This is in line with the research by Isaksson (2012), in which influence from customers was seen to play a significant role in driving LSPs to develop and adopt green initiatives. Other stakeholders who may have an influencing role were a supportive and committed top management, and an environmental awareness and willingness to change among employees, was also mentioned as important when integrating and adopting green initiatives within the company. In addition to the influence of different stakeholders, the research by Isaksson (2012) reveals additional factors acting as drivers for the adoption of green initiatives, see Table 9 below. 
Table 9 Examples of drivers towards the adoption of green initiatives (source Isaksson, 2012, p.51).

\begin{tabular}{llcc}
\hline Potential drivers & N & Mean & $\begin{array}{c}\text { Std. } \\
\text { Deviation }\end{array}$ \\
\hline Cost reduction for customers & 59 & 3.5 & 1.32 \\
Cost reductions for suppliers/partners & 56 & 3.1 & 1.37 \\
Cost reductions within my company & 59 & 3.9 & 1.26 \\
Improve customer relationship & 60 & 4.3 & 0.86 \\
Improve customer service & 59 & 4.1 & 1.10 \\
Increase firm's competiveness & 59 & 4.4 & 0.88 \\
Increase firm's revenue & 59 & 3.9 & 1.21 \\
Increase ROI & 44 & 3.4 & 1.37 \\
Increase market shares & 58 & 4.0 & 1.15 \\
National and EU laws and regulations & 59 & 4.0 & 1.21 \\
Economic means of control (e.g. Taxes, & 59 & 3.4 & 1.37 \\
subventions) & & & \\
Improve brand image & 59 & 4.5 & 0.90 \\
Reduce company risk & 56 & 3.6 & 1.37 \\
\hline
\end{tabular}

The result shown by Isaksson (2012) indicates that adoption of green initiatives can be seen as a way to retain and increase an LSP's competiveness as well as enhance its brand image. In addition, it can also be viewed as a way to improve customer relationships. However, one fact worth mentioning is that not all LSPs may be influenced by the same type of drivers. This may depend on the characteristics of the LSP as well as its surrounding environment. One example of this can be found in the study by Gammelgaard et al. (2012). The study aims to investigate driving forces towards the development of sustainable logistics services within the Danish logistics sector. The results reveal a certain lack of interest among Danish LSPs for developing sustainable logistics services, and few respondents have translated sustainability into their logistic services and made it available on the market. It was seen more as a way to reduce energy and fuel consumption and they do not view sustainability as a way to increase their competiveness. However, the relevance of cost saving was also mentioned in the study by Isaksson (2012) but it was not perceived as one of the strongest drivers when adopting green initiatives among the LSPs studied.

Further, based on the results from their study, Gammelgaard et al. (2012) question whether the development of sustainable logistics services represents a hidden and unseen potential among some LSPs. The authors suggest potential explanations for the findings, such as "lack of visibility of the potential for using sustainability proactively in developing new services" and "lack of knowledge of how a company can transform itself into a sustainable logistics company, and from there, how it can develop new services based on these changes" (p.184). The latter explanation is due to the fact that the industry consists mainly of small and medium-size companies that may not have the same possibilities or focus as a large LSP company to develop sustainable new services.

However, the lack of knowledge of how to become more sustainable is not surprising since this is also a completely new market area for LSPs, who will have to find their own ways to solve environmental challenges and meet customers' future green demands. In addition to lack of green knowledge, the study by Isaksson (2012) reveals some other barriers that may explain the low prevalence of development of green service within LSPs. The most prominent barriers towards adoption of green initiatives concern financial aspects such as high 
investment costs, uncertain payback period as well as lack of economic incentives. In addition, the customers were also seen as a barrier due to their lack of support and an unwillingness to pay more for green logistics services.

Regarding low support and interest in green initiatives on the part of customers, similar results can be found in the study by Wolf and Seuring (2010), which reveals that in performance metrics, traditional requirements such as price, quality and delivery reliability are valued higher than requirements for sustainability. The authors believe that this is considered more as a "hygiene factor" or as add-ons for doing business. Possible explanations for this, according to Gammelgaard and Prockl (2012), apart from companies continuously focusing on economic aspects, concern the difficulties in quantifying the value of the green initiatives. The authors found that customers have a more positive attitude towards green initiatives as long as they have a positive impact on the economic aspects. In addition, according to a survey study, Nordisk Logistikbarometer, conducted by PostNord (2012), which was based on responses from 460 companies in the Nordic region, $37 \%$ of the respondents experience a conflict between delivery service and environmental logistics solutions. In addition, only $8 \%$ of the responding companies are willing to switch to a more environmental logistics solution if it means that the price would increase by $10 \%$. Another possible reason given for customers' relatively low interest is that manufacturing companies have, until now, been focusing on their internal sustainability and therefore not come so far in the environmental considerations of their transport and logistics services (Gammelgaard \& Prockl, 2012). Hence, now is perhaps the time for LSPs to show their customers the potential benefits that can be achieved by focusing on the environment. For example, according to the annual Third-Party Logistics study (2012), new LSP selection criteria such as fuel efficiency and carbon emissions now play a more significant role in customers' buying decisions. In addition, increasing environmental requirements from customers can also be found in the Nordic survey study by PostNord (2012). The most common requirements are ISO certifications, eco-efficient fuel, environmental documentation as well as having the options of train or boat. The result shows that ISO certification is the most frequent requirement (46\%) and has increased by $18 \%$ since a similar survey study in 2010 . Another requirement that has increased dramatically, from $16 \%$ to $30 \%$ during a two-year period in the study, concerns the requirement for environmental documentation.

Even if environmental concern seems to be becoming an increasingly important topic within businesses, it has not reached its full power to act as a competitive advantage to gain market shares and differentiate one actor from another among LSPs today. However, in the study by Maack (2012), differentiation of services was one approach identified for the strategic development of the environmental management within the company in order to reach the market and distinguish it from its competitors.

However, there is a strong feeling that environmental concerns will gain more managerial attention within the LSP industry in the future (Lieb \& Lieb, 2010) and LSPs themselves seem to be future-oriented and view environmental awareness as a potential way to increase their competiveness (e.g. Gammelgaard \& Prockl, 2012; Isaksson, 2012). Furthermore, sustainability is seen as a "license to do business in the future" according to a report based on a workshop with some executives from the LSP industry (Prockl \& Gammelgard, 2012). The agenda for the workshop was to discuss the role that the LSP industry plays or should play regarding environmental development. 
In sum, based on the above discussion it seems that environmental concerns cannot be neglected by LSPs if they want to stay competitive in the future. In addition, Lieb and Lieb (2010) argue that those LSPs which have already started to develop strategies to adopt green initiatives will be far better prepared than their competitors to meet and respond to possible tougher future regulations and increased green requirements from customers. However, the question remains as to how LSPs can become more sustainable and what is needed in order to develop sustainable logistics services.

\subsection{Aspects influencing the development of green logistics services}

As mentioned earlier, the general level of knowledge within LSPs regarding how best to manage the development of green services seems to be low. Although this area has not been a major focus within research, it is seen as a fruitful area both for academia and practice (Jumandi \& Zailani, 2010).

However, the research by Isaksson (2012) seeks to make an early contribution to this field by combining the knowledge from two fields that are seldom considered simultaneously: sustainable product/service development literature and literature regarding logistics service offerings. This resulted in a framework revealing some aspects of and insights into how to consider sustainability aspects in the development of logistics services, see Figure 5.1 below.

\begin{tabular}{|c|c|c|c|}
\hline Aspects & Benefits (Why involve) & $\begin{array}{l}\text { Considerations in the development of the } \\
\text { offering }\end{array}$ & The offering \\
\hline \multirow{3}{*}{$\begin{array}{l}\text { Customers and } \\
\text { market }\end{array}$} & Identify attractive services & Customer demands as a starting point & Diversified and customised offers \\
\hline & Focus the "right" aspects & Early integration of marketing & Pricing the environmental offering \\
\hline & Develop wanted services & & Customer segmentation \\
\hline \multirow{4}{*}{$\begin{array}{l}\text { Collaboration } \\
\text { Internal }\end{array}$} & Knowledge exchange \& spread & Use cross functional teams & \\
\hline & Efficient problem solving & Include environmental experts & \\
\hline & & Identify collaboration risks & \\
\hline & & Develop methods to handle risks & \\
\hline \multirow[t]{3}{*}{ External } & Improved supplier development & Collaborate with suppliers & Show awareness \\
\hline & Shared data and knowledge & Collaborate with customers & Show willingness to collaborate \\
\hline & Improved sustainability performance & Introduce the collaboration early & \\
\hline \multirow{5}{*}{$\begin{array}{l}\text { Business } \\
\text { management } \\
\text { and strategy }\end{array}$} & $\begin{array}{l}\text { Cascade sustainability throughout the } \\
\text { organisation }\end{array}$ & $\begin{array}{l}\text { Integrate strategic, tactical and operational level cross } \\
\text { the company }\end{array}$ & $\begin{array}{l}\text { Hard to visualise in the offering but might be } \\
\text { done with the use of certain certifications } \\
\text { and standards }\end{array}$ \\
\hline & Increase the environmental benefits & Support from top management & \\
\hline & "Common language" among employees & & \\
\hline & Achieve corporate commitment & & \\
\hline & Increased trustworthiness & & \\
\hline \multirow{4}{*}{ ICT } & Enable customisation & Internal measuring & Provide customised offerings \\
\hline & Meet customer demands & Collect and store environmental data & Display/measure environmental impact \\
\hline & Control of environmental impact & & Facilitate collaboration \\
\hline & Support development of new offerings & & \\
\hline \multirow{2}{*}{$\begin{array}{l}\text { Means of } \\
\text { control }\end{array}$} & Stimulate development & Focus on outcome not method & Use labelling/standards in the offering \\
\hline & Economical advantages & Focus/select e.g. standards & Trustworthy and informative offerings \\
\hline \multirow{4}{*}{$\begin{array}{l}\text { Competence } \\
\text { and knowledge }\end{array}$} & Strengthen skills and knowledge & Training and development & Knowledge as part of the offering \\
\hline & Facilitate increased innovation & Adjust knowledge needs to strategy and ambitions & Trustworthy offers \\
\hline & Maintain and keep competence & $\begin{array}{l}\text { Identify potential knowledge exchange with external } \\
\text { actors }\end{array}$ & \\
\hline & Improved performance & & \\
\hline
\end{tabular}

Figure 5.1 Framework towards green logistics services (source: Isaksson, 2012, first paper in the Licentiate thesis). 
Each of the different identified aspects may in itself have some valuable input but the authors stress the relevance of having a more overall perspective of these aspects in order to create an opportunity for LSPs when developing green logistics services. In addition, this framework can be seen as a source of inspiration for both researchers and LSPs rather than as being fully comprehensive. However, some of the aspects mentioned are also identified in other studies as having an impact when LSPs adopt green initiatives or develop green service offerings.

To take one example, in the study by Lin and Ho (2011), the authors identify organisational, environmental and technological factors as having a positive influence on LSPs' adoption of green initiatives within the company. More specifically, the authors found that the factors of "regulatory pressure, governmental support, organisational support, quality of human resources and relative advantage and compatibility of green practices" all influence LSPs in their willingness to adopt green initiatives (p. 80). In addition, the authors recommend that LSPs focus more on the organisational support, and in particular top management support, in their continued green efforts in the company. This is necessary in order to "encourage employees' green behaviour, make organisational resources easily available for their employees, improve their quality of human resources and organisational learning capabilities and accumulate more environmental knowledge" (p.80). In common with the result by Lin and Ho (2011), many researchers mention the relevance of organisational encouragement and the important role of top management support when adopting and integrating green initiatives within the company. For example, Abareshi and Molla (2013) argue that an LSP's exploitation of green knowledge reflects the support of top management for these kinds of practice. In addition, by establishing necessary routines to control existing or new competencies in logistics operations top management may contribute to improving the green performance of the company. This is also supported by Zailani et al. (2011), who stress the relevance of top management support in order to facilitate implementation of new technology as well as make resources and knowledge more available for employees. In addition, the authors suggest that the creation of a climate that allows and supports knowledge sharing within the organisation, can lead to a higher degree of an LSP's green innovations efforts being realised. When it comes to development of green logistics services, Gammelgaard and Prockl (2012) highlight the need for a more innovator leadership style for this kind of process. In line with the discussion above, the results presented by Rossi et al. (2013) also reveal that the level of commitment towards a green approach can be interpreted by a combination of cultural, structural and integration aspects. Aspects found in the study included employee involvement, a clear embedded environmental strategy and shared objectives through specific metrics and a clear leadership.

However, a strong and supportive top management may not be enough, and LSPs are advised to create strategies in order to overcome and manage environmental issues (Jumandi \& Zailani, 2010). In order to strive for a higher level of green performance, Abareshi and Molla (2013) argue that there is a need for LSPs to incorporate environmental considerations into their strategic decisions process. Despite its mentioned relevance, most of the LSPs which participated in the study by Zailani et al. (2011) do not integrate environmental considerations into their overall business strategy. However, most of the LSPs studied have an ambition to improve their green efforts and share the view that environmental concern is important to the firm's strategy. This can be illustrated by the results in the study by Isaksson et al. (2012), which indicate that the existence of green concerns (both formally and informally) in the LSPs' overall business strategy is positively related to the implementation of green measures. The results also indicate a correlation between the existence of green concerns in the business strategy and the active involvement of top management in managing green initiatives. In 
addition, strategic presence and top management support also seem to be related to crossfunctional green involvement as well as to the existence of specialised environmental-focused functions among the LSPs studied.

Table 10 below gives some examples of different functions that may be involved in managing green initiatives within LSPs. The LSPs studied were asked to rate which functions are involved to a high extent in the company's green initiatives. As the results reveal, top management has the highest mean and is also significantly higher than all the other functions (with the exception of the logistics/transport department). The logistics/transport department, as well as the environmental department, also received a relatively high mean. The logistics/transport department is rated significantly higher than all other functions with the exception of top management and the environmental department. However, the environmental department is only significantly higher than the IT department, supply chain department, finance department and warehouse/terminals.

\begin{tabular}{lccc} 
Table 10 Examples of different functions involved in managing green initiatives. \\
\hline Functions & N & Mean & Std. Dev. \\
\hline Top management & 57 & 4.21 & 1.11 \\
Logistics/transport department & 57 & 4.11 & 1.10 \\
Environmental department & 56 & 3.77 & 1.55 \\
Marketing department & 55 & 3.44 & 1.49 \\
Purchasing department & 54 & 3.39 & 1.56 \\
Sales department & 55 & 3.27 & 1.51 \\
IT department & 53 & 3.15 & 1.61 \\
Supply chain department & 47 & 2.96 & 1.69 \\
Finance department & 52 & 2.81 & 1.65 \\
Warehouse/terminals & 50 & 2.70 & 1.68 \\
\hline
\end{tabular}

In addition, the high level of involvement of top management can mean that LSPs perceive adoption of green initiatives as a matter of strategic importance. Further, the involvement of the IT department received a relatively low mean due to its increasing importance and identified potential for LSPs' operations as well as for service development in general (e.g. Zhou \& Wang, 2012; Pekkarinen \& Ulkuniemi, 2008). The low mean may be due to the fact that many of the LSPs participating in the questionnaire survey are small and/or mediumsized companies and may therefore lack a separate IT department. Another explanation may be, as mentioned in section 4.3.4, that some LSPs outsource their IT activities to an external partner and therefore lose some control and power regarding these kinds of activities. However, there may be a hidden potential for integrating the IT department in the environmental work of LSPs to a larger extent and, further, in the development of green services.

The connection between the organisational structure and sharing of organisational knowledge can, in turn, point to the importance of creating internal processes and structures in order to support the development of green services. For example, Gammelgaard and Prock1 (2012) encourage LSPs to adopt a somewhat formalised model for sustainable logistics innovation in order to look for the whole range of innovation options in a systematised way. Abareshi and Molla (2013) also highlight the relevance of, and need for, routines, practices and processes in their study of when LSPs analyse, integrate and transform different kinds of environmental knowledge in order to improve their green performance. The result from the study indicated 
that those LSPs that adopt effective routines and processes to obtain different kinds of environmental knowledge would gain better capabilities for integrating this kind of information into their logistics operations. In addition, routines such as environmental training programmes and implementation of life cycle analysis can act as facilitators for an LSP's success in transforming different kinds of knowledge and information into green routines within the company. Another aspect, which can be linked to the discussion regarding the need for effective and standardised routines and processes, concerns the issue of measuring environmental impact. According to the result presented by Isaksson (2012), the lack of a standardised method and the weak robustness of these calculations may lead to the scenario that customers may perceive these figures as not accurate enough and therefore not be willing to pay extra for these kinds of service offerings. Similar results can be found in the study by Colicchia et al. (2013), in which the authors stress the need for a definition of a method for measuring and allocating the environmental impact to different customers and suppliers. In addition, clear and transparent information concerning environmental impact in the form of cost savings and $\mathrm{CO}_{2}$ reductions could encourage customers to show more interest and increase the willingness to invest in environmental initiatives connected to their supply chain. Similar results regarding the measuring of environmental impact can be found in the study by Rossi et al. (2013). In addition, the authors stress the need for developing measurements for supporting and translating business and environmental objectives into operational and financial targets.

As mentioned earlier in the discussion, the adoption of different technologies has been identified as an important tool in mitigating environmental impact (Zailani et al., 2011). Technology has been identified as one of the major facilitators for sustainability efforts (Prockl \& Gammelgaard, 2012) and many LSPs have started to use new practices and technologies in the movement towards greener logistics (McKinnon, 2010). In addition, Lin and Ho (2008) reveal that increased clarity and benefits of green practices can facilitate the transfer of technological knowledge within the LSPs and therefore raise the willingness to adopt green innovations within the company. This can also be relevant when LSPs consider to what extent they provide transparency and achieve benefits with the development of green services towards their customers.

Table 11 shows some examples of ICT applications that are used in managing the environmental impact of LSPs transport and logistics operations. The participating LSPs were asked to rate the most used ICT and the result reveals that vehicle routing and scheduling receive the highest mean, which is also significantly higher than all the other applications.

Table 11 Examples of ICT aplications for managing the environmental impact.

\begin{tabular}{lccc}
\hline ICT applications & $\mathbf{N}$ & Mean & Std. Dev. \\
\hline Vehicle routing and scheduling & 55 & 3.65 & 1.53 \\
Transport management systems - TMS & 55 & 3.24 & 1.62 \\
Distribution planning & 56 & 3.16 & 1.68 \\
Purchasing and supply planning & 53 & 2.83 & 1.53 \\
Transport simulation/planning & 55 & 2.82 & 1.62 \\
Tracking and tracing system & 54 & 2.52 & 1.69 \\
Warehouse management & 52 & 2.02 & 1.45 \\
Network/facility location configuration & 50 & 1.92 & 1.34 \\
Inventory management & 53 & 1.89 & 1.35 \\
\hline
\end{tabular}


Moving on to the arguments and reasons why LSPs choose to invest in different kinds of ICT applications to support green initiatives within their company, Table 12 presents some examples of potential arguments. The most common arguments are improve competitiveness and reduce cost of transport and logistics operations and these arguments are also significantly higher than all the other arguments.

Table 12 Examples of arguments for investing in ICT to support green initiatives.

\begin{tabular}{lccc}
\hline Arguments & $\mathbf{N}$ & Mean & Std. Dev. \\
\hline Improve competitiveness & 55 & 4.36 & 0.91 \\
Reduce cost of transport and logistics operations & 57 & 4.16 & 1.15 \\
Improve internal control on environmental performance & 55 & 3.89 & 1.15 \\
Improve overall quality of customer service & 56 & 3.86 & 1.18 \\
Improve response to customer's green needs & 56 & 3.82 & 1.10 \\
Improve service differentiation & 48 & 3.50 & 1.37 \\
\hline
\end{tabular}

From a general perspective, technology can be seen as a facilitator of logistics service innovations (Gammelgaard et al., 2012). In addition, since a green logistics service offering can be seen as an example of a logistics innovation, it is therefore not unrealistic to believe that this factor can have an impact when LSPs develop green services. However, improving service differentiation by investing in ICT that supports green initiatives does not appear to be of any great significance since it scores the lowest mean in Table 12. On the other hand, however, this aspect may already be included in the argument to improve the competitiveness.

In addition, Gammelgaard et al. (2012) also mention technology as a means to facilitate knowledge and network relationships. From an external perspective, Table 13 shows the level of ICT integration with other supply chain participants. Integration with the customers seems to be most common and this factor is significantly higher than all the others. ICT integration with other LSPs seems to be the second most common and is also significantly higher than all the others (with the exception of customers).

Table 13 The level of ICT integration with other supply chain participants.

\begin{tabular}{lccc}
\hline ICT integration in the supply chain & N & Mean & Std. Dev. \\
\hline Our customers & 65 & 2.62 & 1.35 \\
Other logistics service providers & 59 & 2.14 & 1.35 \\
Our customers' customers & 50 & 1.50 & 0.84 \\
Suppliers of our customers & 54 & 1.48 & 0.93 \\
\hline
\end{tabular}

In general, all the different levels of ICT integration in Table 13 reveal relatively low means, and indicate thus that there may be great potential for improvements for LSPs within this area. With similar reasoning to that above, the use of technology and adoption of different ICT applications can also be a way to facilitate sharing of knowledge and improve network relationships from a green perspective. From an internal perspective, on the other hand, one way to increase the level of green knowledge within the company, as well as to improve an LSP's progress in green service offerings, is to focus on the role of the employees. Lin and Ho (2008) suggest that LSPs should encourage and support their employees in environmental activities by providing different kinds of environmental training and education. Gammelgaard and Prockl (2012) also support this, and stress that employees are often the actors that can transform a company's environmental efforts into reality. This is also supported in the 
research by Maack (2012). The results from the study reveal the importance of educating employees within sales and operations in order to increase their knowledge about environmental efforts, especially those efforts connected to the services offerings. The author also suggests the potential major marketing role employees can have towards customers by uncovering some of the intangible benefits connected to an environmental effort or initiative.

However, as mentioned in the study by Abareshi and Molla (2013), in order for an LSP to improve its green performance it may be necessary to adopt knowledge from different kinds of sources. The study shows that the most common way for an LSP to obtain external green knowledge was through participation in environmental training programmes, while the least commonly used routine was to gain information from legislation. In addition, the result also revealed that there is a greater focus on internal than external environmental knowledge. However, the author stresses that LSPs need to improve their ability to value and use both internal and external green information in the process to develop green logistics services. The positive effect of integrating both internal and external knowledge when it comes to general environmental management can be illustrated by Maack (2012). Through involvement in network collaborations and focusing on cross-functional integration and knowledge transfer between the sales and transport planning divisions, the LSP studied perceived outcomes such as cost reductions and a reduction of the environmental impact.

Furthermore, Gammelgaard and Prockl (2012) mention both customers and suppliers as potential sources of innovation and stress at the same time based on the result from their study of the importance of collaboration with supply chain partners when developing sustainable supply chain solutions. Despite the relevance and growing interest regarding collaboration with both customers and suppliers, the study by Colicchia et al. (2013) reveals a certain lack of effective collaborative actions to improve the environmental impact of the supply chain. The authors suggest that the level of collaboration is dependent on each LSP's degree of environmental awareness and strategic decisions.

In addition, Gammelgaard and Prockl (2012) recommend that LSPs do not react solely to customers' demands for sustainable logistic services but that they also develop a more proactive approach for environmental improvements, which in turn may create cost savings and increased business for LSPs themselves. It may be necessary for LSPs not to completely rely on customers' green demands in order to move the green development frontier forwards, not least since many studies reveal a lack of interest on the part of customers towards green initiatives. This can be illustrated in the study by Lin and Ho (2011), which shows that the influence of customer pressure is not a significant factor when LSPs adopt green initiatives. However, customer pressure regarding green behaviour may vary and the authors suggest that this can be associated with the position a company has in the supply chain. In addition, Jumandi and Zailani (2010) stress the relevance of the necessity for both LSPs and their customers to be transparent towards each other in order to use each other's capabilities and find innovative ways for green improvements. The relevance of collaboration and joint efforts is also highlighted in the report by Prockl and Gammelgaard (2012). The report is based on a workshop with some executives from the LSP industry in which they discussed the role that the LSP industry plays or should play in the sustainability business. The LSPs participating in the workshop state that improvement of sustainability issues "cannot be accomplished by one actor alone" and mention that they cooperate with their subcontractors and their customers in improving sustainability issues. However, the LSPs stress at the same time that there is a need for more green communication beyond the required reporting as well as for increasing the transparency and involvement of partners and subcontractors in order to improve 
environmental issues. Further, Wolf and Seuring (2010) stress that this will be a huge challenge, since it concerns not only changing business practices within a single company, but also changing attitudes across the complete supply chain. For these reasons, the authors stress the need for a holistic approach when developing green logistics services in order to reduce the environmental impact as well as to improve collaboration, trust and information exchange among actors in the supply chain.

\subsection{Towards a framework for LSPs' management of green NSD}

As discussed at the beginning of this chapter, integrating environmental management into the service development management can be a fruitful area for LSPs in order to increase competitiveness. Therefore, it may be interesting to investigate and address the strategic relevance of the environmental work and efforts, since this may reveal the role and further ambition of an LSP regarding this area and its integration into the service development process.

- What is the strategic role of environmental work within an LSP company in terms of:

$\circ$ Existence of an environmental strategy?

o Influence and integration into the management of service development?

However, even if it is stated that developing green logistics services does not differ in principle from developing other kinds of logistics services, this area is still in an early phase among LSPs. As mentioned in section 5.1.1, in order to understand how LSPs can integrate environmental issues and green practice into their service development efforts, it may be relevant, as a first step, to investigate the status of an LSP's environmental work. This may involve how the environmental work is organised within the company, green initiatives adopted and also identification of different factors that influence this development both from a positive and negative perspective. This discussion leads to the following survey questions:

- How does an LSP organise and work with its environmental efforts?

- Which factors drive and/or inhibit this development to move further?

These survey questions are seen as underlying explanation factors in increasing the understanding of how an LSP can manage the development of green service offerings. The following survey question, however, aims to reveal more specifically how an LSP organises and structures the integration of environmental concerns towards the development of green service offerings. It also aims to reveal the role of the customers and their environmental demands as well as information regarding competitors' green service development and how this is shared within the company and how it influences the service development process. In addition, the final sub questions also include the existence of internal and external collaboration in order to improve the environmental work linked to the development of service offerings.

- In which ways does an LSP integrate environmental concerns into the development of service offerings?
o Customers and market information

o Internal and external collaboration

As in Chapter 4, these survey questions act as an input for the empirical investigation of the selected LSP in this dissertation. These questions are also used as a means to structure the 
collected empirical data. The overall link between these questions, as well as the link to the general conceptual framework of an LSP's NSD management, is illustrated in Figure 5.2 below.

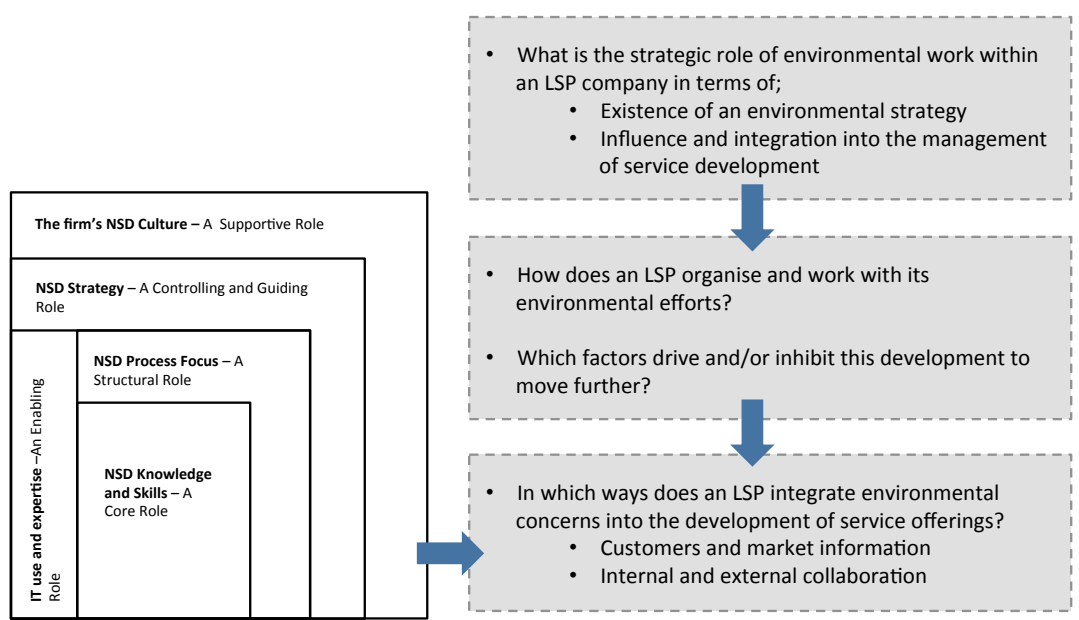

Figure 5.2 The empirical guide for a LSP's management of green NSD.

The aim with Figure 5.2, is to illustrate that in order to investigate the role of green service development and to identify pre-requisites for this development within LSPs, it appears be relevant as a first step to understand LSPs strategic environmental ambitions as well as their existing environmental work. The strategic focus together with the exisitence of green initiatives and environmental knowledge/skills within the company put thus to some extent the base and preconditions of the development of green services. Further, the general service development ability can also inspire and affect in which ways environmental concerns are integrated into the development of LSPs' service offerings. The empirical findings regarding these questions can be found in the second part of the case description in Chapter 6 . 
-94 - 


\section{CHAPTER}

\section{POSTNORD - THE CASE STUDY}

This chapter aims to give an illustration of how an LSP manages and structures its overall service development as well as of its attitude towards the development of green services. The chosen LSP for this dissertation is PostNord (PN) Logistics, and the empirical assembly is mainly based on interviews together with information from annual reports and the company's homepage. This chapter is divided into three different parts. The first part includes a general company presentation of PostNord followed by a description of the PN Logistics' service development management. The second part concerns the environmental work and existence of green service offerings. This part is from a PostNord perspective but with a specific focus on PN Logistics. The third and last part aims to illustrate a new service development project where environmental considerations have an essential role. The main empirical input to this chapter is based on interviews but also includes secondary data derived from the company's homepage, annual reports, company reports as well as internal presentation material. 
- 96 - 


\section{PART I: INTRODUCTION AND SERVICE DEVELOPMENT MANAGEMENT}


- 98 - 


\subsection{The company}

The PostNord AB Group (hereafter referred to as PostNord) was formed through the merger of Post Danmark A/S and Posten AB in 2009. PostNord is a Swedish corporation, owned 40\% by the Danish state and $60 \%$ by the Swedish state. The company offers mail and advertising services in Sweden and Denmark and is a leading provider of logistics services to, from and within the Nordic region. The market presence focuses mainly on the Nordic region, but through the Strålfors business area, the Group offers information logistics service throughout a number of other European countries. In 2012, PostNord reached net sales of approximately SEK 39 billion and the company has over 40,000 employees.

As mentioned above, PostNord was originally two national postal operators active on two separate monopoly markets. Today, however, the company views itself as a Nordic service company "active in a competitive international area". Like many other LSPs, PostNord's business is affected by the changing market conditions such as increased globalisation, new communication opportunities through the digitisation trend, as well as enhanced focus on the environmental impact. The company's awareness of these structural changes and the understanding of its continued impact on the future business environment can be illustrated in their core values:

\section{"the reliable, accessible and environmentally sound business partner for communication and logistics (PostNord Annual Report, 2012, p.1)”}

\subsubsection{The business scope of PostNord}

This section aims to give a brief presentation of PostNord's different business areas in order to give the reader an insight into the scope of PostNord's business. PostNord has operating activities in three different business areas (BA): Mail, Logistics and Strålfors, see Figure 6.1.

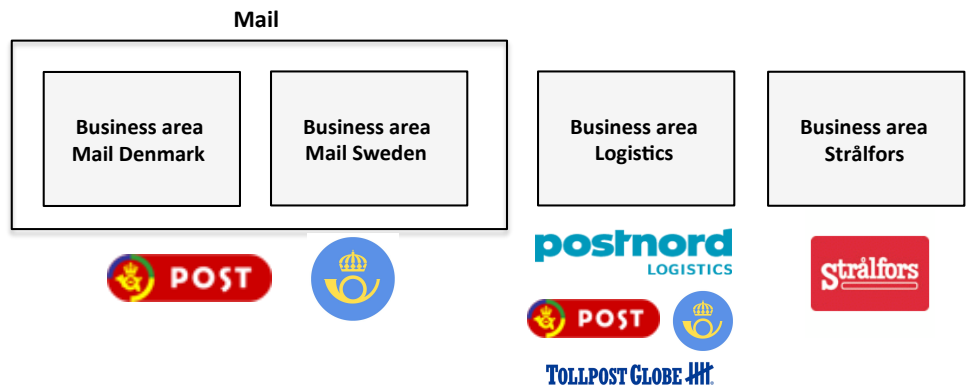

Figure 6.1 PostNord's different business areas (source: PostNord Annual Report, 2012).

As Figure 6.1 illustrates, the Mail business area is divided into two areas: Mail Denmark, which operates primarily under the Post Denmark brand, and Mail Sweden, which operates under the Posten brand. This business area offers services for business and consumers with a focus on business communication and other mail services for marketing communications. BA Mail holds a leading position within these areas in both Sweden and Denmark.

BA Logistics offers logistics services with a focus on parcel and pallet distribution, together with solutions for companies' logistics flows within the Nordic region. This business area 
holds approximately $10 \%$ of the Nordic market, which makes Logistics one of the largest logistics operators within this region. The business of Logistics is primarily driven under the Posten, Post Denmark and Tollpost Globe brands, but will progressively be marketed under one main brand: PostNord Logistics. BA Logistics will hereafter be referred to as PN Logistics. A more detailed description of PN Logistics can be found in the next section, since this business area is the main focus of the empirical study in this dissertation.

The third and final business area, Strålfors, operates in the area of information logistics. The main focus area of Strålfors is Business Communication, Data Management, Marketing Communication and Service Fulfilment. The core customers consist mainly of companies with a large customer base who want to improve communication solutions to their own customers. Strålfors is primarily present in the Nordic region, where it holds a market-leading position, but it also has additional operations in other countries, such as the UK, France and Poland.

\subsection{Business area PN Logistics}

PostNord is a leading operator on the Nordic logistics market, offering its customers a Nordic-wide distribution network. PN Logistics is divided into different operational units, which in turn are divided into countries and companies linked to the business area, as illustrated in Figure 6.2. The different operational units are primarily responsible for running and managing the operating activities. In addition, two functional units, Market \& Sales and Finance \& Operations, support and follow the development of these operational units. The overall responsibility of these functional units is to manage joint development, optimisation and coordination of existing and new business within the different countries. The management of PN Logistics consists of the Business Area Manager, representatives responsible for each of the functional units as well as managers responsible for the operational units in Sweden, Norway and Denmark.

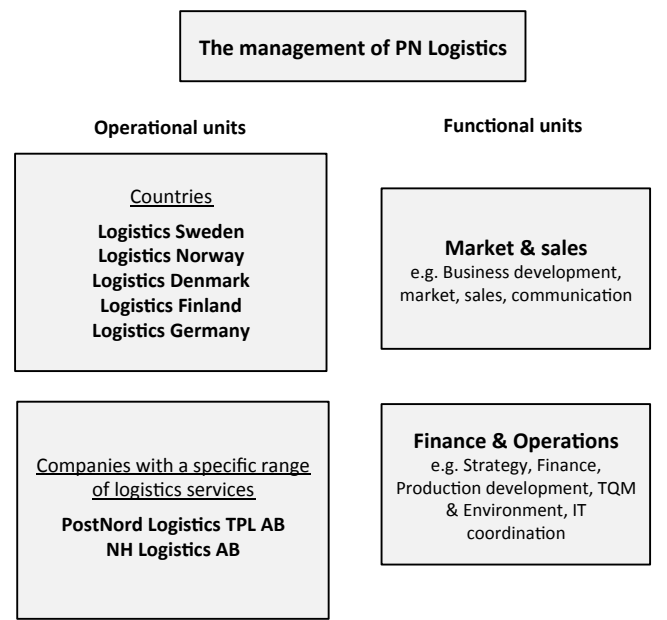

Figure 6.2 Structural organisation of PN Logistics business area (source: PostNord). 


\subsubsection{The overall strategic development and business model of PN Logistics}

One of the strategies of PostNord's Roadmap 2015 is to continue its development within the PN Logistics business area with the aim of reaching a market leading position in the Nordic region. PostNord strives to make continued efforts and investments in order to broaden the Group's product offerings and market presence in the Nordic region. This will be achieved by organic growth through additional sales and partnership and potential acquisitions. The business model of PN Logistics and its strategic focus is illustrated in Figure 6.3 below.

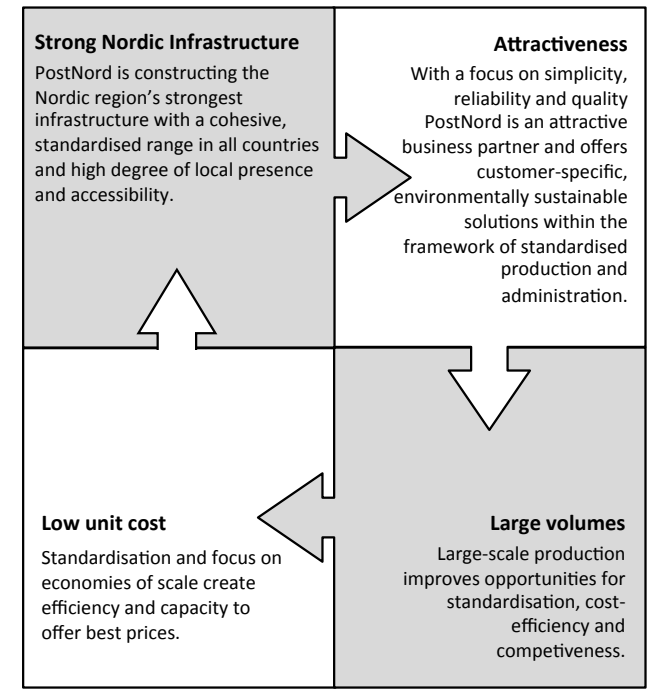

Figure 6.3 The Business Model of PN Logistics (source: PostNord Annual report, 2012).

During 2012, PostNord made several efforts to broaden and standardise the logistics offering, enhance strength in selected submarkets and expand the geographic coverage. This was done in order to improve opportunities for additional growth and provide services and end-to-end solutions that better match customer demands. For example, PostNord made or initiated three major acquisitions during 2012: Green Cargo Logistics, Harlem Transport and Byrknes Auto. Other recent major investments have concerned, for example, PostNord's establishment of a new combined parcel and pallet terminal, and investments related to vehicles and handheld scanners in order to streamline production efforts and create possibilities for capacity expansion. In addition, in its annual report PostNord stresses the relevance of environmental awareness for becoming a leading logistics operator. Thus, PostNord has engaged in efforts in the development of alternative fuels and new vehicle technology with the aim of reducing carbon dioxide emissions (PostNord, Annual Report, 2012).

\subsubsection{The range of service offerings and position on the market}

PostNord's logistics business offers a wide range of logistics services and runs operations in parcel, pallet and mixed cargo groupage as well as delivery, express, third-party logistics, night freight forwarding and consignment freight (PostNord Annual Report, 2011). PN Logistics has divided the logistics market into nine different sub-segments that illustrate the range of the business area services:

- Courier

- InNight

- Express 
- Part and full Loads

- Groupage/Pallet

- Parcel B2B

- Parcel B2C

- Air \& Ocean

- TPL

During 2011, the business area developed and standardised the Nordic products, which are service offerings common for all the operational units in the different countries. These services are managed and developed jointly within the business area. Figure 6.4 illustrates PN Logistic's overall service offering range and also reveals the Nordic products. Today, these Nordic products consist of four different basic services:

- B2B (business to business) parcel;

- B2C (business to consumers) parcel;

- B2B pallet; and

- InNight

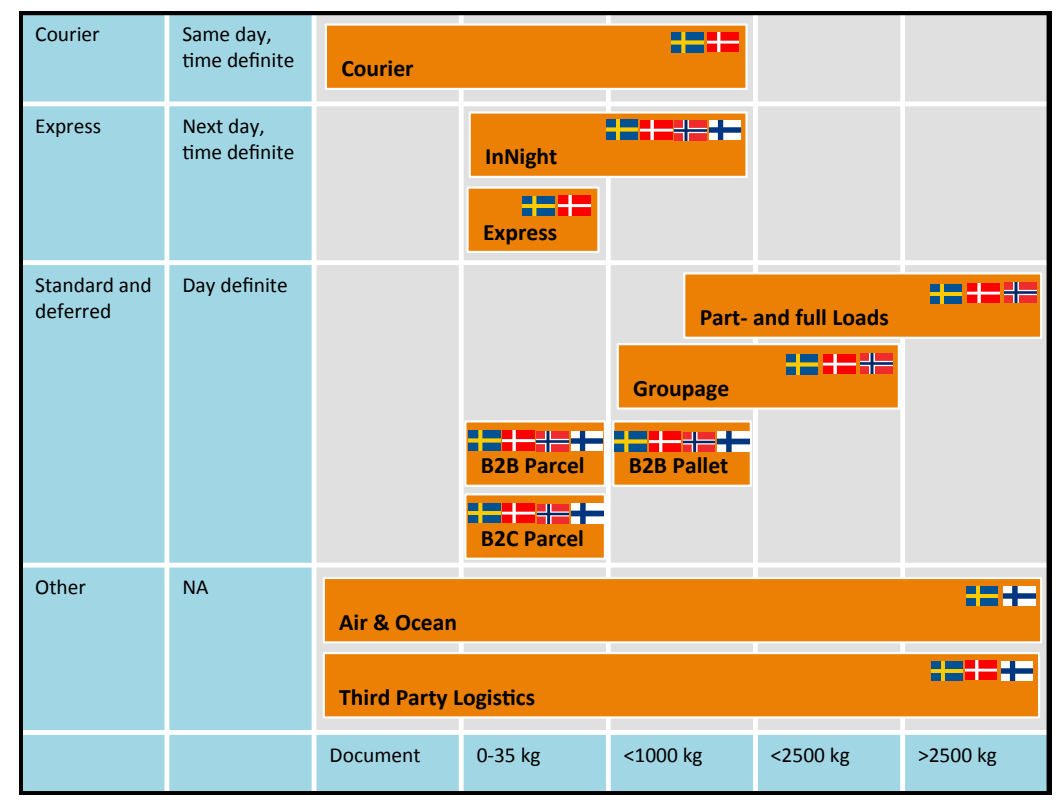

Figure 6.4 PN Logistics' range of service offerings (source: PostNord, 2012).

These Nordic products can be complemented with a number of additional services and can also be combined in different ways. The reason for this chosen approach is explained by PN Logistics, below:

"It is very much about improving the already existing service offering and adding customer value by combining different additional services and becoming more transparent about, for example, environmental data. This approach will also be easier to understand from a customer perspective." (Vice President, Business Development, PN Logistics, 
Currently, the focus is on the sub-segments Groupage and Part and Full Load in order to make these basic services more interesting in the Nordic countries. PN Logistics has identified an untapped potential to increase the growth within these areas, and there is also a desire to be transnational when it comes to these services. In having these Nordic products in place, PN Logistics will have a comprehensive logistics service offering in the Nordic region.

In order to be referred to as a Nordic product, these service offerings must fulfil a number of requirements. The requirements consist of four different directives. Firstly, the basic characteristics of the service offering must be comparable in need, dimensions, maximum weight and basic responsibility. Secondly, there must already be common additional services in all countries, e.g. SMS or letter notification. In Sweden and Denmark, however, there are also some unique additional services for these countries. Thirdly, the price structure must be reasonably coherent. The price structure is very complex, and any change made may have a negative effect on profitability. The final requirement is that the name of the service must be similar in all the different countries.

As has already been mentioned, there are also some additional services linked to these Nordic products, which may differ slightly between the operational units in the different countries. The service development for these Nordic products can be divided into two parts: one part which is common for the whole business area and another which is unique for the operational units. This means that the different operational units are responsible for the additional services that are unique in their own country and the operational units are also the product owner of the services that are not connected to the Nordic products.

The position of PN Logistics in the Nordic market depends on which sub-segment is in focus. In some areas, e.g. B2C parcel and B2B parcel, PN Logistics has a leading position, based on revenue and volume, while in other sub-segments, the market positions are not so strong. However, overall, PostNord is a top player in the Nordic countries, based on revenues, with approximately $10 \%$ of the market.

\subsubsection{Customer segmentation}

The majority of the sales consist of credit customers who sign a contract with PN Logistics. Only a few percentages of the sales consist of cash sales. Moreover, the main part of this consists of companies, which tells that a very small percentage of the revenue comes from private customers.

The segmentation of PN Logistics' customers is made through a classification based on revenue generation. The classification includes the following levels: KAM customers, large customers, medium-sized customers, small customers and customers who pay in cash. The KAM customers are relatively few but they represent a major share of the revenue. Overall, there is a very large number of small customers, especially in Sweden and Denmark (around 30,000 in Sweden and almost as many in Denmark).

In addition to the segmentation on the generated revenue, this classification also reflects the type of sales channels used for the different groups of customers. In order to be cost effective, the approach for reaching and communicating with these customer segments differs. For example, the large customer segment is approached by different key account teams, which work together with the customers within a variety of areas such as safety and environment. Meanwhile, the small customer segment is approached by telephone sales. 
The structure of the following subsections is inspired by the conceptual model and includes NSD constructs presented in Chapter 3. The outline of these subsections is illustrated in Figure 6.5 below.

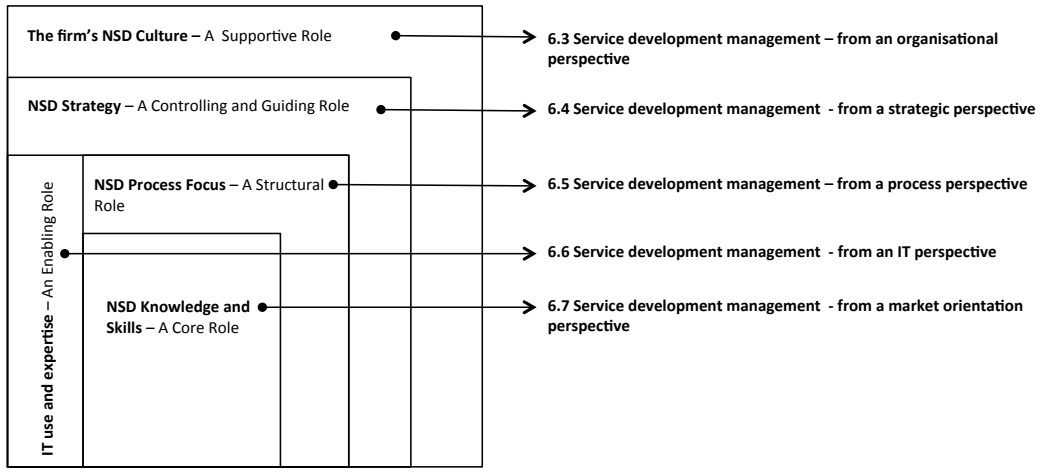

Figure 6.5 The outline of the subsections 6.3-6.7.

However, the labels of the different subsections do not directly correspond with the NSD constructs included in the model due to the content of the different subsections. For example subsection 6.7 aims mainly to represent the construct NSD Knowledge and Skills in the model, although additional relevant aspects regarding this construct are also integrated in the other subsections.

\subsection{Service development management within PN Logistics - from an organisational perspective}

In total, around 20 employees work in the Market and Sales department. The manager of Market \& Sales has a functional responsibility for the unit and is ultimately responsible for the service development concerning PN Logistics in all the Nordic markets. The functional unit, see Figure 6.6 below, in turn consists of the areas of Sales, Communication \& Market, Business and Market Intelligence and Nordic Services. The service development is well integrated on a Group level, which in turn takes decisions such as what is most important and what needs to be prioritised. Issues that are discussed concern, for example, how to deliver organic growth, results and how to deliver on the set growth strategy.

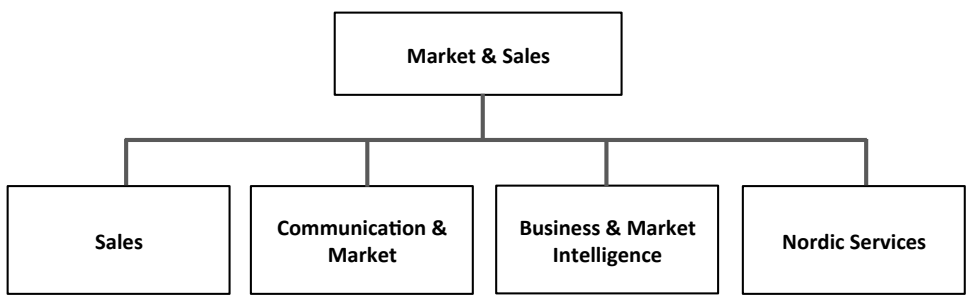

Figure 6.6 The structure of the functional unit Market and Sales (source: PostNord, 2012).

In general, the service development process does not differ considerably between the different sub-segments within the business area. Still, PN Logistics plans to recruit four employees under Market \& Sales who will focus on service ownership and service development on a business area level. This in turn will require input from various sources and collaboration 
efforts between the areas concerned. Responsibility for the service development will not lie solely with these four employees. Rather, there are plans to form different teams, each with the right skills needed for the specific area. For example, these teams might include employees from production, IT and also employees working with the billing of services.

At the strategic level, the R\&D function is integrated in the functional unit Market and Sales. The aim of the function is to have a good knowledge of the market, insights into existing competition and market analysis, as well as conclusions on the continued development and future trends. Each business area has its own R\&D function. The way in which the different business areas work with their business development will differ slightly, but they are basically very similar. However, one example of a structured cooperation between the different business areas is connected to the customer segment that is linked to the e-commerce business. In this area, logistics and mail services meet similar customer needs and must therefore be linked to each other. This initiative has targeted the Group level, and intensive efforts have been made in this area in order to continue to bundle different kinds of services towards this customer segment. In addition to this, there have been a number of streamlining activities between the various business areas' R\&D departments. However, each business area operates in different types of markets, hence the need for separate R\&D departments.

From an organisational perspective, the management of and responsibility for the service development within PN Logistics differs between the countries. In Sweden, a relatively large department works with service development. In Norway, the service development is integrated in the overall business activities. Nevertheless, there are some employees who work more intensively with business development, even though there is no product owner or special unit that owns and develops services. This is the case in Sweden, Denmark and Finland. Despite this, PN Logistics aims to have a more uniform structure of service development within the different countries. Regarding service development on a Nordic level, four employees are dedicated to this, in addition to the Business Development Manager.

\subsubsection{Collaboration between the different operational units}

Through various forums, representatives from the operation units meet and discuss how activities could be better coordinated between the different countries. This applies not only to areas as service development, but also sales activities. Issues brought up to discussion can concern the development of a common CRM strategy, partnerships with customers, business development with customers, how should this work be analysed and what goals should be set.

As mentioned earlier, the operational unit in Norway has a different approach towards organising service development; the work is to a larger extent integrated in the overall business. This approach is influenced by the historical development and the culture that has prevailed within the company. In Sweden and Denmark, which were originally old postal authorities, the culture has been more strongly influenced by structured processes and stringent requirements to achieve the set goals. Norway does not have the same structure. Despite this, PN Logistics can identify advantages when these two different cultures meet:

"This can allow initiatives to be taken at an earlier stage and to dare to test in a small scale, but at the same time should be rely on a certain structure." (Vice President, Business Development, PN Logistics, 15 January 2013)

In addition, Norway is perceived to be customer-focused in everything they do, which in turn affects the control of the development, organisation, and management priorities as well as the 
culture and attitude. They listen to the voice of the customers, which facilitates the process of making the organisation prioritise correctly. Norway has developed and worked systematically with customer relationship management for about 10 years, which has resulted in the concept of an "Advisory Board". The success of this concept can depend on many parameters but customer surveys reveal high rates when it comes to loyal customers and a strong relationship and dialogue with the customers.

\subsubsection{Information sharing}

In order to spread and share information within the organisation, PostNord uses something called "dialogues". The aim of the dialogues is to create a basis for the managers in the form of a film or a map, which the managers then present for their employees. In this way, all managers present the same information in a similar manner. The information presented is then discussed within the group and thereafter reported into the intranet. This concept has been used several times, for example at the presentation of PostNord's core values. Previous employee surveys showed a lack of trust for the Group level, as well as confusion concerning the core values of PostNord. However, this has improved through the dialogues. Other means of communication used to share and spread information are PostNord's own internal magazine and the intranet. There are two kinds of intranet channels: one for the managers, who in certain situations can be provided with information earlier than other employees, and another for all employees, where, for example, different types of tools and information sheets can be found.

\subsection{Service development management within PN Logistics - from a strategic perspective}

The top management team is responsible for the formulation of the service strategy. The design and the content of the strategy depend on the current company position and are based on a thorough market analysis of the Nordic region. Inputs to this analysis include:

- Mapping the markets - divided by products and the different countries;

- Customer insight - specific customer requirements, how large the volumes of these customers are, as well as future-oriented analysis regarding customers' bargaining power regarding products and services within markets where PostNord is not present today;

- Overall analysis of the competitors - services offered by competitors and sales for each of PostNord's business areas;

- Profitability - the progress of the economic development in the future and use of financial resources as efficiently as possible;

- Analysis of the current situation - environmental and other technical regulations as well as specific customer requirements that are relevant.

The business area's service strategy, which can also be seen as the growth strategy, is perceived to be aligned with the overall business strategy of PostNord, which in turn has a high focus on investments. The correlation with the overall strategy is based on the fact that PN Logistics has to fulfil a number of requirements, e.g. reach a certain level of growth within specific segments at the same time as maintaining good profitability. Acquisitions and organic growth are important leverage factors for PN Logistics. However, it is important that these investments support the growth strategy, and can be integrated in the business 
operations as well as having synergies with existing operations. This strategy is followed-up quarterly and action plans are presented for those areas that do not fulfil the set requirements.

The service strategy applies to the entire business unit but there is also one unique strategy in each operational unit in the different countries. The strategy has a strong growth focus and includes aspects such as focused product segments and growth plans for segments. For every operational unit in the different countries, there are directives concerning which strategic investments should be made and how the operational activities should be managed. In addition, realistic targets are set for each country regarding the service development. These targets include, for example, the sales for each country in the different business areas and also the sales and market position to be reached at the end of the strategy period. These aspects form the overall business unit strategy for PN Logistics but also work at the same time as its service strategy.

The service strategy is seen as the controlling part, but there are other strategies that support this strategy. The strategy regarding development of services is fully aligned with the service strategy and examples of other supporting strategies are connected to the production and sales area. The production strategy concerns the entire group, and involves issues related to the infrastructure and optimisation routines. The connection between the production strategy and the service development strategy can be formulated in such a way that the production strategy sets the stage and provides opportunities as well as limitations for the service development strategy. For example, when it comes to transport capacity, decisions are made regarding the degree to which this capacity should be held in-house vs. outsourced. These levels are set on an overall level and create the pre-requisites for the service development efforts. For example, a pre-requisite is created if decisions are taken to rent capacity in order to meet market fluctuations or differences on a daily basis, where the decision to have an over-capacity in order to be able to take extra customer assignments is set. The over-capacity in turn creates incentives for what kind of service can be developed in order to sell those extra transportation hours.

In parallel with the standardised and long-term development of the production, the market department receives input from customers, which can initiate possible adjustments or streamlining of the production. One example of this is the service logistics that requires installations inside the customer's premises compared to shipments just to the "door". This has not previously been done within PN Logistics. This requires development of new additional services, new information sharing platforms and education for the drivers in order to increase their knowledge regarding these kinds of service offerings. These service offerings require adjustments that may not be in line with PostNord's overall strategic and efficiency focus. However, there is a need to move towards the market as well as consider the "time to market" issue. By testing different ideas from customers, the service development is pushed forward and possibly commoditised in the future to make the service offerings available for several customers.

Another strategically important part is connected to communication measures. In order to deliver the growth strategy, PN Logistics emphasises the issues such as:

"It is important to have a living brand and, in order to facilitate the communication, how should we describe ourselves, what industries should we focus on and grow within and how can we try to become partner aligned with customers?" (Vice President, Business

Development, PN Logistics, 15 January 2013) 
The different countries have multiple brands linked to their business, the consequence of which is that the PN Logistics business area as a whole has to handle around 15 different brands in its portfolio. The number of brands will most likely continue to increase in the future due to acquisitions. Owing to strong competition, organic growth will not be enough and the company will instead need to acquire a large number of companies along the way in order to reach the growth goals. PostNord aims to become the leading logistics player in the Nordic region, which means a requirement to be perceived and viewed as the "number one" player in the market. As an important step in this work, PN Logistics plans to implement one main brand during 2013, which will be applied for the entire business unit. This master brand will be called PostNord Logistics. Thus, the companies acquired will eventually be fully integrated into PostNord's product portfolio.

\subsubsection{Requirements when developing new service offerings}

In order to develop a new kind of basic service, the market segment must represent many billions of SEK. The ambition is to standardise the services to as large an extent as possible. The development process can be initiated by a specific requirement from one customer but PN Logistics strives to make the service available to a wider customer base, from a costbeneficial perspective. Generally speaking, profitability in the transport and logistics industry is obtained by economies of scale. Furthermore, too many special solutions can increase the risk of mistakes in the process due to the number of people who are involved in the delivery process of the service.

The optimal scenario in the development of additional services is that these services can be combined with all the basic services. However, some of these additional services are unique to a particular basic service. On the question of whether or not to expand its product portfolio, PN Logistics believes that the first step is to grow with existing customers and further develop already existing services. PN Logistics aims to create a range of different basic services with a variety of additional services in order to make it possible to combine these according to the specific needs of the customers. This is done in order to increase the degree of standardisation and also reduce the unit cost in order to achieve profitability and also to be able to offer competitive prices to its customers. Another important aspect is to develop a service offering that is easily understood by the customers. However, this is not a unique situation for PN Logistics. Rather, it is about offering the customers solutions and presenting a more solutionoriented than a product-oriented sales process. The goal is to sell a solution which fulfils the specific customers' needs, developed by existing building blocks, in order to gain efficiency in the production mechanism.

There is no upper limit for how many of these additional services should be developed. PN Logistics would like as many additional services as possible. The unlimited number of additional services depends on the concept of simple basic services. These basic services are relatively unadorned but the idea is that they can be extended with a variety of additional services in order to be able to meet a specific customer's needs. This concept of basic services and additional services is also a way for PN Logistics to focus less on services and thus devote more time to finding a customised solution for a specific customer.

From a traditional point of view, PostNord is characterised as a strong production-oriented organisation but strives to become more customer-oriented in its operations. Collaboration with customers has existed in the past, especially connected to strategic initiatives that have had an important impact on the business as a whole. Nevertheless, one stated strategy within PostNord is to continue to grow together with existing customers. Exactly how this should be 
done is not clear, and this issue is something that applies to all the different business areas. In order to gather insight, a number of key customers have been identified and a target group has been selected in order to focus on and find potential solutions that go beyond the boundaries of the different business area. In addition, tracking of the sales and how this is developed towards the customers is also monitored closely. This Group-initiated activity involves both the business area managers and key account managers from the different business areas, and is thus a cross-functional collaboration. Corresponding activities with key customers are also conducted within the PN Logistics business area. The approach to the activity differs between the operational units in the different countries depending on how far the countries have come in this work. In general, there is a desire not to be perceived as a subcontractor to the customer; instead PN Logistics desires to develop a closer relationship with the customers and become a "partner". In order to create this relationship, a number of customers have been identified and work is done to actively monitor sales performance towards these customers. These customers are selected due to the fact that they have operations in many countries, since this is an area where PN Logistics believes it has potential to grow. Due to the customers' transnational operations, work is done for sales people in Sweden, Norway, Denmark and Finland in order to get to know each other and share knowledge. Other examples of efforts to try and standardise the sales work are creating tools and systems that are available when needed, ensuring a quick decision-making process and more actively offering training to the sales people. This is undertaken to make the sales people think more holistically and to become better at capturing the needs of customers and finding solutions to these specific needs. Even if no standardised solution has been developed yet, the business area is willing to invest money and develop customised solutions.

\subsubsection{Identifying new ideas, evaluating and closing down existing service offerings}

Recent figures show that the mail volumes are falling and the CEO of PostNord has been instructed to make the company more publicly ready, which in turn will lead to growth and profitable requirements. Within the mail business, efforts are being made to find hybrid and advertising services in order to cover the loss of the declining mail volumes. The challenge for the PN Logistics business area is to create a strong and substantial growth in the coming years in order to deliver on the five-year corporate strategy. The market areas where PN Logistics has a strong position today have no potential for continued growth. This is due to an already high market share, and instead the business area must enter new market segments where no service offerings exist today. These kinds of service initiatives are strategically driven and require PN Logistics to create capability to provide customers with services in the heavier freight areas where PostNord is present on the market but not viewed as a strong actor. However, even if growth within new areas is viewed as important, PN Logistics states that:

\section{"The most important thing is to grow with our customers." (Vice President, Business} Development, PN Logistics, 27 December 2012)

The scope of such initiatives ranges from small to large. From a bottom-up perspective, it may concern the development of various additional services based on specific customer needs identified by the sales organisation. Meanwhile, from a top-down approach, it may concern how PN Logistics can become a strong actor and reach a market position within areas such as parcel and pallet goods. 
Adding additional services and efforts to improve the entire service offering is something that PN Logistics sees as a matter of course. There are some limits related to the development of services, such as dimensions and maximum weight, which is equal in all the countries and cannot be changed. PN Logistics has an active dialogue with stakeholders and a structured approach when identifying new ideas of what to invest in or not to go forward with. However, this process is not always without obstacles:

“...you always have to make some priorities when it comes to internal resources as well as IT development resources." (Vice President, Business Development, PN Logistics,

27 December 2012)

Several of the major strategic initiatives that have been established are based on a dialogue with the customers and their needs. As mentioned earlier, in Norway, there is something called the "Advisory Board", where customers have the opportunity to express their needs and wishes? This approach of working with customers is considered successful and is to be integrated within the business area in Sweden as well as the other countries. The strategy builds on activities such as customer visits as well as different types of local workshops with customers regarding areas such as the environment, quality measurement and other relevant and valuable issues for the customers. A larger meeting with the customers is arranged annually in order to follow up this work and discuss what has been done during the year and also the further plans. This approach is considered to be a good way to build strong relationships with the customers.

Within PN Logistics today, there is on-going work with major customers. Meetings to discuss different focus areas, such as operational, tactical and strategic issues, are held on a regular basis. The approach with different focus areas in the meetings is intended to meet the "right" audience at the customer company. These meetings often result in various issues that must be addressed and further developed.

When it comes to evaluating the service offerings developed, PN Logistics follows the profitability of the service group level, for example for Parcel B2B, and also the growth of the market. Market research is also done, where customers have the opportunity to say how the service offerings of PN Logistics are perceived.

Decisions about whether certain services should be terminated or further developed are often based on a business case approach. Other criteria that must be satisfied in order to keep an existing service offering are sufficient volumes and profitability. PN Logistics strives to make the service offerings more attractive, easier and simpler for the customers. Therefore, phasing out certain services which, for example, meet similar customer needs, is seen as an important part of PN Logistics' work regarding service development. This is done not only due to the fact that too broad a variety of service offerings can lead to an unclear picture of the service offerings to the customers but also that too big a product portfolio entails high costs.

\subsection{Service development management within PN Logistics - from a process perspective}

PostNord has a set plan and structure regarding how the general service development process should proceed. This plan or structure is in line with how PN Logistics actually works with its service development efforts within the business unit. The plan is set at the Group level and 
consists of three main processes: develop services, sell services and produce services. The plan for developing services consists of several logical steps, one example of which is to identify and evaluate customer needs. Furthermore, within the process, some services are closed down while others are further experimented upon. The process supports the development of both existing and new services. The alignment of these main processes and their role in the PN Logistics business process is illustrated in Figure 6.7 below.

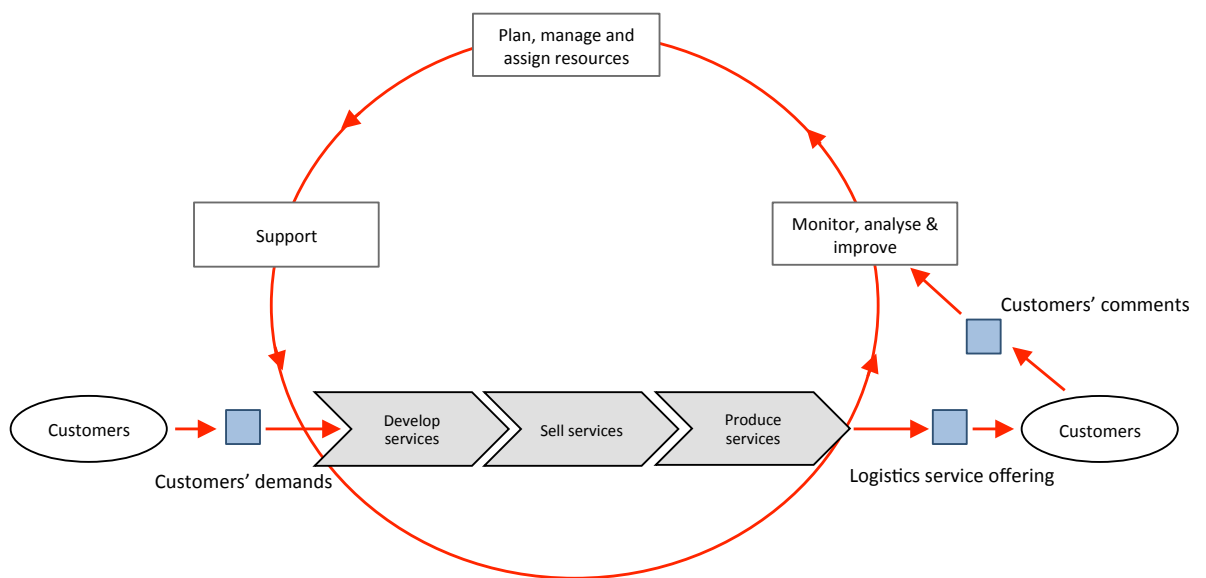

Figure 6.7 The Business process of PN Logistics (source: PostNord, 2012).

Within the develop services area, PN Logistics adopts a process model of how to proceed in the development of services and there is also an ambition to implement this model in the rest of the operational units in the different countries.

The three different process areas are not seen as separate parts. Instead, for example in the ongoing work with the introduction of parcel goods, teams are formed with employees from each area. The different skills needed for this work are collected in the same project team. These employees have joint workshops and are seen as central for the future work of the project.

\subsubsection{Existence of structured procedures and supporting tools}

PostNord has a development methodology as well as a project methodology, which serves as support and as a tool in the development of projects and services. This methodology is the same for the entire group and is used in the development of both new services and technical solutions. The development methodology can be seen as an overall process methodology. This process starts with an idea, followed by a number of tollgates (around five to six decision points), which have to be addressed in order to move further to the next stage. Moreover, when a project starts, different roles have already been identified, as well as when these should be integrated into the process in order to address different parts of the project. In other words, a "team composition" is created where each player or employee has a specific purpose or role in the different parts of the project.

"This makes it very clear when to start working, which in turn makes the time to get started with the project relatively short."(Product Specialist, Market \& Sales, PN Logistics, 11 October 2012) 
The project methodology can be used as a complement to this process, especially when it comes to larger investments. The project methodology consists of a number of templates and checklists. However, the existing project methodology is to be replaced, partly due to the fact that it is considered somewhat old-fashioned. There is a desire to change the approach of working, to make it more agile, have a greater degree of testing and form project groups in a different way.

However, the replacement of the project methodology is creating some confusion among the employees. PN Logistics is currently about to start a new project, where the new project methodology will be applied. This is a huge initiative, where the strategic parts will be handled and driven through major projects while the minor and more operative parts will become more daily changes. Both the major projects and the minor operational changes follow specific process steps. It is a very structured process, for example when different types of suggestions of solutions are developed. In addition, PN Logistics states that:

“..we are extremely structured, sometimes maybe a little too structured when it comes to how to run minor changes. Especially if the change is connected to IT, then the process becomes very structured. It can involve, for example, who makes decisions, what kind of order is it, how should the testing be approached, who will accept delivery and so on." (Vice President, Business Development, PN Logistics, 27 December 2012)

\subsubsection{From idea generation to launching of the service concept}

The main drivers for developing new services are based on customers' demands as well as competitors' developments. For example, within the market department of PN Logistics Sweden, customer inquiries represent the primary reason for driving the service development work further. Every week, the market department receives several calls from customers and then takes decisions about which customers' requests should be further developed. The decisions regarding which requests should be further developed are based on whether this seems to be relevant from a strategic or solution-oriented perspective. The market department employs five product developers or specialists who work together, discuss and brainstorm different ideas regarding various service offerings proposals. This process differs from the production development process, since the process requires quick decision-making and the ability to seize the moment with the customers. This also requires a good knowledge of the company's strategy and where it is heading, as well as keeping track of the outcome and results of the customer surveys in order to be able to push the service development further. The product developers or specialists are "responsible" for different product areas. However, internal discussions are held in order to exchange knowledge and ideas. Moreover, additional knowledge is gained through customer visits, but also internally by practising on the terminal in order to increase the knowledge of the production and its mechanisms.

As mentioned earlier, more or less everything is driven from the customers; they contact the market department and inquire whether PN Logistics Sweden can come up with a solution to their problem. Based on a specific customer's inquiries and requests, an internal discussion takes place in order to come up with ideas and suggestions for what can be done for the customer. Thereafter, the relevance of these suggestions and ideas is tested in a "test bench" before running a project regarding this issue. If the outcome of the project is positive, this means proceeding in the process onto the next stage of formalising and productifying the service offering. 
When a service concept is to be tested, regardless of whether it is a new service or not, this is done, if possible, to a limited extent. This is done to be able to evaluate if the project should move further or not and this approach is used regardless of whether a new machine is being invested in or if a new service is going to be developed. Tests are done within a limited area and during a limited time horizon. Within PN Logistics Sweden, there are three new projects where tests are either being done with a particular customer or within a particular geographical area with a few drivers acting as test subjects. Based on the results from the tests, attention and focus is given to which kinds of investments are needed. The identification of investment cost is a very important step since it can otherwise lead to dramatic consequences.

In addition, when it comes to the strategic development efforts regarding the production, market and service development area, the strategies connected to these areas have different time perspectives. For example, the production strategy is further advanced compared to the service development area, largely because of structure decisions. Today PostNord is building new terminals in Sweden which have an investment cost of several billion. These investments are not being made for any specific customer. Instead, PostNord considers it to be an area worth long-term investment. From that perspective, the production strategy and service development strategy are not aligned time-wise. However, at the same time there is an overall pressure to reduce the time to market by finding smart, effective and low-cost routines and solutions. Based on this, there are requirements to improve the speed of service development efforts and also be more keen to change in the display and interface to the customers. Efforts are being made to identify and map what is available today and how this can be put together; in other words, some kind of "model building" thinking, based on the prevailing conditions. Existing data and systems are used from a production perspective but will perhaps be complemented or modified with a new time for delivery or an additional service. Since PostNord has a relatively good spread of day and night distributions, this "model building" thinking is a way to constantly broaden the range of service offerings quickly to customers.

In addition, if a new investment is made in order to solve an internal process in the production, efforts are made to integrate the market and service development parts. This is done in order to identify the needs and requirements from customers and integrate these into the process. There are some cooperation efforts between the different functions within PN Logistics Sweden. Previously, there has been little cooperation between production and market, although they have identified that they want to work more together.

\subsection{Service development management within PN Logistics - from an IT perspective}

Almost regardless of what type of service development it is, service development will require some type of IT change and usually also require some type of change in the production. The involvement of IT expertise in the development of services is done to a large extent since the service offering more or less consists of an IT solution. Approximately $90 \%$ of the service development consists of IT development, and only a smaller amount of process changes in the production. Overall, when a change of a service is made, this will to some extent affect IT support.

However, since PN Logistics has such a large production mechanism, it places requirements on structure and thorough investigations about what it is possible to do. For example, if a 
change is to be made in the extradition process to private individuals, this change will affect about 30,000 employees who work with delivering packages. This change will thus require a huge educational effort, as will be the case if a change is made among the drivers who are involved in distributing goods. It is not possible to make any change unless the drivers' PDAs (Personal Digital Assistant) support it. These things must be taken into consideration because it is production that essentially represents the service offering.

\section{"If you want to solve a certain need, there is never just one way to solve it, so you have to set up a number of options against each other. You simply have to make some kind of business cases and try to determine if you will get the money back on the investment that you make." Vice President, Business Development, PN Logistics, 27 December 2012)}

PostNord has a very fragmented portfolio of systems within the organisation, yet investments have been made in order to build a common IT system for all the different countries. One example of this is the Track and Trace solution, which facilitates the process of tracking the package in order to find out where it is and when it will be delivered. In the past, there have been different types of solutions in order to receive this kind of information in the different countries. However, this investment is decided at Group level, since PN Logistics is not the only business area that uses this service. The idea behind this initiative is that it will provide the same type of information in all the different countries. Other joint investments are a Nordic transportation database that shows the distance and how long it takes to ship a package between two addresses, common freight documents, as well as a common system for measuring the quality that is cross-border and applies to all countries.

Whether or not there are any restrictions in the IT support connected to service development, it is often a matter of fact of making priorities.

“...you always have higher ambitions than you have time to implement. Resources are available but these are of course not unlimited." (Vice President, Business Development, PN Logistics, 15 January 2013)

PN Logistics has outsourced a part of the operation and development of IT, which has partly resulted in a perception of getting less for the money, and also that it takes longer. This is considered as a challenge.

In addition, regarding their own intranet, there are opportunities for improvements, both internally but also in the interface to the customer. PN Logistics believes that the process of disseminating information can be improved; however, these efforts require certain investments to be made within IT. Once again it is all about priorities; nevertheless, PN Logistics sees the distribution and provision of information as an important part of the service offering. It is perceived as a value-adding component (which does not directly generate revenue), but can in turn increase the customers' willingness to pay and increase customer value. Building a greater electronic connection with customers can lead to a stronger relationship. 


\subsection{Service development management within PN Logistics - from a market orientation perspective}

Within PN Logistics there is a structured dialogue on how to take care of customer needs. There are joint meetings and discussions about the customer needs that have been identified and PN Logistics has a long list of what the business area wants to do. Once a year, a proper screening of the identified customer needs is carried out in order to prioritise the different customer needs and evaluate what has been done during the year. However, there are occasions when the priority order changes depending on what kind of urgent customer needs arise during the year. In addition, information from customer support as well as information from complaints is also seen as an important input to service developments. Information based on customer support, complaints and sales is documented and matched with the strategic initiatives. This kind of information can serve as a base in order to reach a particular customer segment or industry. Developing a clearer industry focus as well as industry solutions is something that PN Logistics has started to consider.

\subsubsection{Market analysis as an input to service development}

By working in teams, PN Logistics tries to use the information from the market analysis in as efficient way as possible. A variety of competences are present within these teams, ranging from skills regarding service development, marketing and communications at a Nordic level.

"This is seen as a huge strength as well as the importance of the informal communication between the people involved in these teams." (Vice President, Business Development, PN Logistics, 27 December 2012)

From time to time customers are invited. One example of using input from different actors is the work connected with reverse logistics, which is an area that has recently been developed and changed with particular regard to everything that is happening within the e-commerce business. New kinds of customer needs have started to emerge. As transports have become more international, the question of returns has become more complex, especially if these involve customs. As an input to this work, a dialogue has been held with partners and other actors involved such as developers of TA systems (Transport Administration system). Moreover, specific customers have also been invited to participate in the discussions and PN Logistics has also made reference visits at the customers' companies. Through internal discussions, the information from the different parties has been organised in order to develop a number of initiatives that may have positive impact. Reverse logistics is an example of an initiative which is in the borderland of a bottom-up and a strategic perspective and is an area that has the potential to be developed in the future.

PN Logistics tries to integrate the customers during the service development process, especially when it comes to the strategic initiatives. The customer must somehow be involved all the way and be a natural phase in the process; it is when the customer becomes more involved that the various pilot tests are performed. These tests are always performed together with the customer in order to identify if something needs to be corrected or added. Customer participation is seen as a necessity when things need to be tested at different stages in the process since it is hard to predict all possible outcomes in advance.

\subsubsection{Identifying new customers and monitoring the competitors}

The approach to finding new customers is quite structured and the focus is currently on finding a more industry-specific approach to identify new potential buyers. PN Logistics 
believes it offers good solutions within some industries and aims therefore to reach other companies within these kinds of industries. These customers are reached either by using the existing portfolio of potential customers or by using existing databases where customers have been divided into different sectors/industries and segments in order to find "twins" to existing customers. Due to the fact that PN Logistics can offer more complete solutions than before, this leads to new business opportunities with customers who were previously interested in these kinds of solutions. However, making this work successful and implementing it in a structured manner, requires access to developed IT support on the CRM side. Due to the size of the organisation and involvement of hundreds of sales people, the work has to be done in a systematic and structured way. Within the organisation, some operational units within the different countries are better than others when it comes to the process of identifying potential new customers. However, PN Logistics aims to develop a uniformed way of conducting this work and form a strategy that will be applied to all the countries in the Nordic region. This is being done in order to take advantage of each other's competencies in the entire organisation.

The PN Logistics business area is working actively to monitor the progress of its competitors. This is done partly with input from customers, but also based on input from publications such as press releases and new customer cases. Another annual input is companies' publications of new price levels and terms. A number of major competitors have been selected in each market for more careful monitoring through evaluations and measurements. They include Schenker, DHL, Bring, Itella and DSV. Through a competitiveness index, structured measurements are made of these players in order to evaluate PN Logistics' development in comparison. This kind of information will not only work as an input to service development but also to areas such as customer service, complaints and delivery quality. This information also reveals the different areas customers perceive as important. In addition, PN Logistics highlights that:

\section{“A similar evaluation could also be used in order to investigate customers' interest and perception regarding the environment. Factors such as price and delivery quality are known to be important aspects for customers but the importance of the environment among customers is somewhat unclear." (Vice President, Business Development, PN Logistics, 27 December 2012)}

However, the attitude regarding the environment among the customers can vary within the different product areas. A more detailed description of PN Logistics' environmental work and its connection to its services can be found in part II of the case description.

In addition, it is observed how customers value PostNord's position against their competitors. These measurements have a broader scope and do not only include PN Logistics' existing customers. For example, if the results reveal that competitors achieve better results, a further analysis is done in order to find out the reasons for these results.

\subsubsection{External actors as an inspiration source for service development}

Within the transport and logistics industry, Schenker is seen as a successful actor when it comes to designing and launching service offerings to the customers. This is something that can be further improved within PN Logistics. In addition, another issue mentioned regarding service development concerns the time to the market. The speed of the service development process is currently perceived to be too slow. However, in order to speed up this process, inspirations from the telecom industry are mentioned, due to the ability of those actors to change billing models and speed up the process from idea to action. 
"The ability to be more effective in the process from an idea to action is something that PN Logistics should strive for and be better at managing." (Vice President, Business

Development, PN Logistics, 27 December 2012)

However, the issues regarding the speed of the service development process depend partly on the fact that the organisation is very fragmented and consists of many different countries, which all have their own systems. It will be a very costly process to change all of these systems, both from a cost and time perspective. In addition, there is perceived to be a certain inertia from the IT department, which has requirements from the Group level to reduce costs. These requirements do not have a positive effect on increasing the speed of the service development process. 
-118 - 


\section{PART II: ENVIRONMENTAL WORK AND GREEN SERVICE OFFERINGS}


-120 - 


\subsection{Environmental work at PostNord}

In 2009, at the time of the merger between Posten AB and Post Danmark, it was clear that both postal operators had a solid environmental performance. However, Posten AB had progressed slightly further than Post Danmark regarding integration of the environmental issues into the operations. Nevertheless, both Posten AB and Post Danmark had high environmental ambitions connected to their activities. Earlier, within Posten AB, a scorecard was created containing both financial and non-financial key performance indicators, which are still in use within PostNord. One of these key performance indicators concerned the environmental impact in relation to turnover. It was questioned whether this ratio really gave value to the customers or internally in the organisation. This led to the development of other indicators to monitor or measure the environmental impact of the operations. This work resulted in a number of new key performance indicators, which concerned grams of carbon dioxide per letter (first class mail) and grams of carbon dioxide per package. Furthermore, there are also more operational measurements such as diesel/10km or $\mathrm{kWh} / \mathrm{m} 2$ that are more informative for managers and employees out in the field.

The primary reason for the diesel/10 km measurement may not be environmental, but rather cost, due to the fact that fuel is a large fixed cost in a transport-intensive enterprise. However, reducing the amount of diesel per $10 \mathrm{~km}$ has a positive environmental effect and it is also easy to communicate the metric, as well as also create participation and involvement around it.

In addition to the operational and relative environmental targets, there are also long-term environmental goals (established in 2009). When determining these long-term environmental goals, a thorough analysis was done. This analysis involved input from the various business areas of what they thought they could achieve by 2020 in order to reach PostNord's overall goal in 2020. This work led to the long-term goal that PostNord would reduce carbon emissions in absolute terms by $40 \%$ from 2009 to 2020 . This is a challenging goal, but it is not unattainable. The different business areas themselves set the operative environmental goals and they also decide on the goals.

However, there is an aim for continuity in the overall environmental goals. Also, the relative goals and the performance indicators are to a large extent fixed. However, the set goals are discussed annually based on the outcome. The business plans are formed based on a threeyear period and if the outcome is not going as expected the environmental target levels are revised. The operational goals are not changed very often either; however, the target levels are revised annually based on the outcome of these operational goals. A valid reason for a revision of the goals, which include a lower level of ambition, is required, since this is an issue of credibility and transparency to both internal and external stakeholders.

PostNord tries to be as transparent as possible when it comes to communicating the environmental objectives of the company to its customers.

\section{"From a general point of view, PostNord could become better at communicating all environmental work we actually do." (Environmental Manager, PostNord,} 27 December 2012)

However, there is an on-going effort to, for example, mark the DME cars, electric hybrids, in order to use the trucks as an "advertising pillar" for the environmental work. 
In the environmental calculations provided to the customers, there is transparency regarding how they have been calculated and which assumptions have been made. However, one problem that PostNord has noticed regarding these environmental calculations is the fact that different players on the market make these calculations differently. For example, both DHL and Schenker calculate $\mathrm{CO}_{2}$ per tonne kilometre, which PostNord does not. So when customers ask for $\mathrm{CO}_{2}$ per tonne kilometre, this rather complicates things since PostNord cannot really develop it in a simple way.

PostNord uses default values when calculating the environmental impact of its customers' transports, which do not provide the accuracy that the customers might have wished. Achieving accuracy would require a more detailed follow-up than is done today, as well as vehicular computers in all cars. For large customers that have nationwide transports, the calculation is fairly accurate, while for the smaller customers, the results may be a little misleading. However, using templates to calculate the environmental impact is something that most of the players within the industry apply. But PostNord has seen that the customers want to know the exact impact on the environment from their transportations. Conversely, the environmental manager of PostNord states that:

"It is possible to become more accurate if you focus on heavier segments where there is more of a transport systems arrangement where you drive for one customer all the time."

(Environmental Manager, PostNord, 27 December 2012)

\subsubsection{Environmental initiatives adopted within PostNord}

Achieving the environmental goals will require a variety of actions, such as running on more energy-efficient modes of transport (nearly $65 \%$ of the mail volume is already running on the train today), and building new terminals in order to be able to increase mail volumes on trains. Within the PN Logistics business area, opportunities have been identified to run more pallets and packages on trains for long distances in order to reduce road traffic. All these initiatives have a major effect on the environment and are therefore seen as a key element to achieving the $40 \%$ target. Another environmental initiative is connected to the opportunity to use alternative fuels. It is easier to find alternative vehicles within the Mail Sweden business area, due to the use of more light vehicles. A large number of electric vehicles are used within the postal operations today. This is a proportion that has increased over the years and PostNord has today one of Europe's largest electric vehicle fleets among postal operators. Within the Swedish postal operations, every fourth vehicle is an electric vehicle. However, when it comes to heavier vehicles, it is more difficult to find alternative fuels or, indeed, find alternative vehicles at all (at least for heavy trucks with trailer). PostNord tests almost everything available on the market, and since the company is a big actor on the market, PostNord is an interesting test "partner" for both Volvo and Scania.

In recent years, PostNord has tested DME (Dimethyl Ether) trucks and hybrid electric vehicles, and in 2013 tests will be done with cars running on methane gas. Both within the heavy truck and postal operations area, tests with vehicle computers exist in order to monitor the drivers' behaviour. Training drivers and postmen in eco-driving is also an important part of the work. Other environmental initiatives adopted include route optimisation and increased fill rate. In Sweden, the environmental progress is perceived as being a step ahead of the development in, for example, Denmark. This insight has also been found in some customer surveys, which have shown that the environmental market progress in Denmark has not been as great as it has in Sweden. 
"This difference depends to a large extent on external factors, which have influenced and set the pace for environmental work in the various companies within PostNord." (Environmental Manager, PostNord, 27 December 2012)

\subsubsection{Driving forces regarding the environmental work}

Looking at the stakeholder picture of PostNord, there are expectations from all the stakeholders to encourage environmental issues in a serious and proper manner. There should be a consequence of what is said and done. Employees are a very important part in this work, as is the ability to attract new employees, which is more value-driven. This becomes more of a sustainability perspective, which does not only include environmental issues. Furthermore, the owners set very tough environmental demands. The PostNord Group is owned $60 \%$ by the Swedish government and $40 \%$ by the Danish government. Both owners have very farreaching expectations of PostNord as a state-owned company. The Swedish government came up with new requirements for state-owned companies regarding their sustainability efforts before the summer of 2012, so there are quite high expectations that PostNord's sustainability work will be at the forefront.

In addition, PostNord believes that the environmental work will strengthen the brand of the company.

"If you are a Nordic leader in your industry, you also have to be a leader in environmental issues. You have to be proactive in the development and somewhere it is believed that it will strengthen the company's image and brand." (Product Specialist, Market \& Sales, PN Logistics, 11 October 2012)

When it comes to customers' requirements, there are some public procurement cases which have high environmental requirements. This puts strong pressure on PostNord to be far ahead in the development of environmental work. These requirements are tough. However, it is worse when a large nationwide company makes environmental demands, since PostNord may be unable to meet them. Nevertheless, PostNord can still present a plan for how it will improve in regard of the environmental standards set. Despite this, customers still want information about the environment. Many of the environmental issues or requirements that customers want to discuss come down to a fairly operational level and involve specifications regarding types of tiers, alternative fuels for transports as well as environmentally classified vehicles. This is something PostNord perceives itself to be better at than its competitors, since some of these competitors may not place the same demands on their suppliers, e.g. foreign suppliers, who perhaps do not have an environmentally-friendly vehicle fleet.

PostNord has its own vehicle fleet and uses this fleet all the way to the customers. This is done in order to control and manage the production unit and thus ensure that everything from quality to environmental issues is taken into account. Another driving force behind the environmental work is connected to financial issues.

"If you can reduce fuel consumption by 5\%, that is a lot of money. Also, if you can increase the filling rate of all cars this can lead to big savings for the company." (Environmental

Manager, PN Logistics, 11 October 2012)

\subsubsection{Barriers regarding the environmental work}

Regarding the barriers connected to PostNord's environmental work, one concerns the environmentally-friendly vehicles and the availability of these on the market. Due to 
investments regarding normal-sized electric cars, this option is perceived as too expensive as it stands today. Although these cars have lower maintenance costs, it is not cost-effective to buy a fleet of electric cars. PostNord is currently involved in a project that is examining what role electric cars will have in the future and what will be needed in the form of incentives and instruments. In Germany, the electric car fleet has increased enormously in recent years, largely because it is subsidised by the German state, which is pushing the development forward in a different way.

Another barrier is connected to the infrastructure development, which is not perceived as matching or supporting PostNord's own environmental development regarding, for example, the presence of charging stations for electric cars. Furthermore, work on looking into new types of fuel is on-going; all options, however, are more expensive than the fuels used today. It is possible that there will need to be some form of subsidy from the Swedish government in order to drive this development further. There are several options on the market, which in turn leads to questions such as:

\section{“..should you invest in a lot of different types of fuels or should you invest in only one type of fuel?" (Environmental Manager, PN Logistics, 11 October 2012)}

PostNord is involved in a project concerning DME cars, which includes about ten test cars in Sweden, and a small pilot plant in Piteå. However, this is a very expensive system and raises questions such as who should pay for the costs and who is going to build the infrastructure to fuel these vehicles.

Research and development has been undertaken within the area of fuel but it must be commercially justifiable as well. PostNord perceives that there is a strong level of motivation among fuel producers and vehicle manufactures regarding these issues but it will be expensive. Regarding the demand from customers, in some cases the customers ask for two different prices, one standard price and one green price. The B2B customers are very priceconscious but PostNord has perceived a difference when it comes to end users via Internet, when these users are going to choose and select shipping alternatives. These customers tend to be more willing to pay a slightly higher price.

\subsubsection{Environmental concerns - a potential competitive advantage}

PostNord sees the environment as a future competitive advantage, but it will probably be required to be more clearly integrated in the sales process. The sales people need to become confident in talking about environmental issues with customers without bringing in an environmental specialist. However, this scenario looks slightly different in the different business areas. Some of the business areas have come a long way regarding educating the sales force in these types of issues. The Mail Sweden business area has come a long way in this work, which is quite natural since they already have bundled environmental services within the Climate Economic product family.

"In order to be able to sell these kinds of service offerings, you have to understand the underlying reasons behind them." (Environmental Manager, PostNord, 27 December 2012)

PostNord wants to incorporate the environmental aspects into its business as a next step in order to sell environmentally-friendly products and services. However, this is an area where PostNord has made great progress in recent years, especially when it comes to being better at presenting the sustainability efforts made to its customers. None of PostNord's competitors 
stand out; instead, all are perceived to have a solid environmental performance. The only difference might be that competitors have been more successful than PostNord at communicating their environmental performance. Nevertheless, none of the competitors is seen as an environmental leader within this area.

\subsection{Organisation of the environmental work}

As mentioned before, PostNord consists of four different business areas: Mail Sweden, Mail Denmark, PN Logistics and Strålfors. These four business areas have reached different levels of maturity regarding breaking down the environmental targets into their own business area. However all the businesses areas are actively working with environmental questions.

The Environmental Manager is responsible for the environment and quality unit within PostNord and therefore has a functional responsibility for the environment and quality work. Within this responsibility, there is one controlling part and one supporting part.

The unit consists of eight employees (five in Sweden and three in Denmark). Furthermore, there is also one employee responsible for the environment within each of the business areas. All these people represent what PostNord refers to as the Environmental Council. The Environment Council discusses and defines environmental key figures and long-term environmental ambitions. For example, the goal to reduce carbon dioxide emissions by $40 \%$ by 2020 was based on a number of different aspects. One of these aspects concerned competitors' environmental targets but the most significant aspect consisted of a thorough analysis of what it is reasonable to assume that PostNord can achieve. The analysis was conducted by the Environment Council.

In 2010, the Climate Fund was established in PostNord, in which funds are earmarked for environmental investments. The size of the Climate Fund is equivalent to what it would cost to offset carbon emissions for the entire Group (Sustainability Report, 2011, PostNord). The employees from the different business areas can submit proposals to the Environmental Council in order to improve environmental and climate measures in their own operations. These proposals of improvements are discussed within the Environmental Council and are then put forward to the Climate Fund. The Climate Fund consists of the Vice President, the Communications Manager of the Group and the Environmental Manager.

The applications received and the Environmental Council's recommendations for a decision are discussed within the Climate Fund. The extent of the proposals received can vary, and concern areas such as leasing of methane cars to replacing lighting in offices.

Some years, not all earmarked funds have been used and there is an aim to focus on more major projects and applications. However, many of the current proposals are of a minor nature, which in itself is positive, since one of the aims of the Climate Fund was to create participation and commitment within the organisation. The Climate Fund gives an opportunity to invest in initiatives that would probably not have been conducted otherwise, or to bring forward investments. For example, PN Logistic's operational unit in Norway, Tollpost, applied for funds to replace doors in order to better keep the cold out. The Climate Fund is seen as an enabler to achieve the $40 \%$ target, but much of the environmental work concerns the daily work to increase fill rate, reduce empty returns and route optimisation. This work 
may not have a direct link to the environment; instead, it is seen as a way to make logistics more efficient with a positive environmental impact.

\subsection{Green service offerings and customers' green requirements}

The customers' environmental requirements vary between the different business areas. Within Mail Sweden, the customers, who are usually public organisations, have very tough environmental requirements. The progress made by different business areas varies when it comes to providing various types of green service offerings. For example, Mail Sweden has developed a product family of green service offerings, which is called Economic Climate. This business area has had a different approach regarding environmental issues and also has other conditions, as well as other customer opportunities than, for example, Logistics. PostNord places high requirements on their customers which they must meet in order to be able to buy these kinds of services. Mail Denmark also has climate service offerings, but quite low volumes on these compared with the Economic Climate services within Mail Sweden. The reasons for the low volumes are somewhat unclear but some factors that may have influenced this are:

"Small and medium-sized customers in Denmark are perhaps not fully aware of environmental requirements but it can also be because the sales people have not sold it in the same manner as Mail Sweden, whose sales people are very active, with the result that these kinds of services will also be sold. " (Environmental Manager, PostNord, 27 December 2012)

\subsubsection{Environmental work and green service offerings within PN Logistics}

As mentioned at the beginning of this chapter, the core value of PostNord is to be an available and reliable business partner and also to be environmentally-friendly in its operations. These core values should in the highest degree characterise the service offerings as well as the service development process. The work to integrate these values in the service development is to a large extent based on requests and needs from the customers. For example, when it comes to the environmental values, a need to provide environmental reports to the customers has been identified. PostNord has in the past chosen not to offer its customers specific green service offerings, even though there are some green added services within PN Logistics Sweden. There are, however, some thoughts on possibly starting to offer specific green service offerings to the customers in the future. Meanwhile, a reduced impact on the environment should not have too great an effect on the service and cost levels. PN Logistics is, therefore, struggling with questions such as how these green service offerings would be designed and which criteria would need to be fulfilled.

“...the main focus cannot just be about carbon offset; instead it must contain something of real value for the customer. You have to make real actions out of your promises and also make demands on the customers as well. On the other hand, being more effective from a green perspective usually goes hand in hand with reduced costs as well. For example, by increasing the fill rates, positive outcomes will be reached both from a green and a cost perspective." (Vice President, Business Development, PN Logistics, 27 December 2012)

In order to integrate green aspects more into the business, PN Logistics has recently started to work on establishing a new brand for the business unit where environmental friendliness will be one of the core values. Much environmental work has been done previously within PN Logistics but it has not really been reflected in the daily work, on the service side. 
No specific green service offerings have been developed within PN Logistics other than offering customers emission reporting, if they ask for such a report. In the past, PostNord's strategy has been to try to improve the environmental performance within the entire business and it has therefore hoped that customers have been satisfied with this. Furthermore, the business area has not previously experienced demands from customers for specific green service offerings. The attitude of the customers is that they want to improve their environmental performance and reduce emissions but they are not willing to pay for it.

\section{"In negotiations with the customer, it is important that, as a logistics service provider, you reach a certain environmental limit; but in the end it's the price that matters." \\ (Environmental Manager, PN Logistics, 11 October 2012)}

Moreover, within PN Logistics other types of environmental initiatives to become more environmental friendly are in place, such as fleet changes and also offering training to the drivers regarding eco-driving. There is a variety of underlying activities to improve environmental performance, although this is something that is not always mentioned or shown to customers.

As mentioned earlier, within PN Logistics, the customers require reporting regarding the environmental impact of their transports and also to some extent environmental certification. Nevertheless, the requirement regarding environmental certification has decreased, and is instead seen among customers more as a hygiene factor. The customers' knowledge about the environment is perceived to vary somewhat. Some customers who undertake robust environmental work of their own are perceived to be very well informed and to know exactly what they want and why. Other customers are not perceived to have the same level of environmental knowledge when demanding environmental documentation.

\subsubsection{Efforts for bringing the business areas together and developing green service offerings}

At the moment, PostNord is working to consolidate the different business areas and discuss service development jointly from an environmental perspective. This work does not include exactly what form service or product will take. Instead, aspects such as which characteristics green services should consist of and how the framework should be designed are being discussed. All business areas are involved in this work and the ambition is to present a proposal to the Group management. Linked to this work, a programme called Young Professionals was initiated that investigated the opportunities of green services within the logistics area.

“..the programme resulted in a number of good ideas where many proposals concerned the communications part since much of the work is about packaging and communicating what we already do. It cannot just be about carbon offsetting, there must be some significant value for the customers." (Environmental Manager, PostNord, 27 December 2012)

The overall aim of this work was to develop a common set of environmental criteria which the different business areas could relate to in their own service development processes. This produces benefits through the use of each other's knowledge of green expertise and service development skills. PostNord perceives that their customers are no longer satisfied with the answer that PostNord works with environmental questions in general. Customers have started demanding specific green service offerings, which means that PostNord has to meet these requirements. However, the general environment work is an approach that PostNord cannot 
let go of since this carries the risk of not achieving the set environmental targets; yet the company must also begin to meet customer requirements for specific green service offerings. PostNord has to find the balance to do both.

From an overall service development perspective, it is all about meeting existing customers' needs and moving forward together with these customers. The incentives for PostNord's internal environmental work to reduce its environmental impact are slightly different compared to the incentives for developing green service offerings. The incentive for developing green service offerings is to meet customers' needs and also to tie the customers closer to the company. For example, this can be seen in the case where Mail Sweden sells Climate Economic services to existing customers. The customer is given a sticker tag, which can be used for advertising and marketing purposes and shows that the customer is working to reduce climate change in collaboration with PostNord.

PostNord has realised that the environmental aspects are becoming increasingly important and has also noticed increased requirements with regard to environmental reporting, since customers want to know the effect of their transports on the environment. However, many customers are satisfied with a figure corresponding to $\mathrm{CO}_{2}$ emissions, and that they understand the logic of how it is calculated. Some problems may arise for some customers when they buy transport services from multiple logistics operators, which many customers in the logistics area do. This leads to customers receiving figures that cannot be summarised since different logistics operators calculate the environmental numbers differently. PostNord tries to counteract this scenario by being engaged in various industry forums in order to provide uniform environmental calculations, which makes it possible for the customers to compare logistics operators and see which is the most environmentally efficient.

"We need a standard way to calculate the environmental impact of transports in order to
make it possible for customers to compare their choices and it is only then a lower
environmental impact can determine the choice between two actors." (Environmental Manager, PostNord, 27 December 2012)

Today, the customers are perceived as putting price first and delivery quality second. The results of customer surveys show that there is no direct willingness among customers to pay extra for green service offerings and further, that they will not accept that it takes longer to deliver their products.

\subsubsection{Customer segmentation based on environmental requirements}

Presently, there is no structured work regarding customer segmentation with respect to the environment. Nevertheless, it is possible to execute, and this approach has been tested and implemented for PostNord's largest customers.

\section{"Much of this work, however, is based on what you know about the customers and also based on sure instinct." (Environmental Manager, PostNord, 27 December 2012)}

This work is not based on any scientific research showing an accurate result, but it gives an indication of what environmental requirements customers need. The chosen customers were placed on a scale with environmental performance on one axis and environmental image on the other. With this scale, it was possible to group those customers who had a good environmental performance and a genuine environmental work and those customers who had a high environmental image. Furthermore, based on a similar analysis, it is possible to adapt 
the communication for the customer target group and try to package different types of service offerings. It can also be seen as a way of helping customers that have good environmental performance to also improve their environmental image. This will result in an active dialogue and sales effort to tie the customer closer and also to strengthen the whole affair.

"This is something that is not discussed to a large extent within PostNord, however, each business area could go on with this work with, for example, the top 20 largest customers within each business area." (Environmental Manager, PostNord, 27 December 2012)

\subsubsection{Communication regarding environmental efforts to customers}

Within the business areas of Mail Sweden and PN Logistics, work has been done through Environmental Manager to Environmental Manager meetings. Present at these meetings are representatives from the customer companies as well as representatives from PostNord. The sustainability manager or the environmental manager from the customer company is given the opportunity to describe their environmental expectations regarding PostNord as a subcontractor and also express their view of whether PostNord lives up to these requirements or not. The purpose of these meetings is to spread the customer company's environmental work within its own company. Furthermore, PostNord's sales force also finds it easier to take up a more environment-related discussion with the contact person at the customer company and therefore finds it easier to sell green service offerings that can further improve the customer's environmental work. It is not certain that the contact people at the customer company itself have green expertise or have any sense of what their group wants regarding environmental issues.

\subsubsection{External inputs for the environmental work}

Customer surveys do exist and are carried out by PostNord, in which some parts concern environmental issues and the customers have the opportunity to rate PostNord's environmental performance. There are also competitive surveys that include environmental issues and these surveys are sent out to general logistics buyers (not only to existing customers of PostNord). These surveys are conducted annually. The information from the surveys are used more or less actively since it involves the entire operation, ranging from how customers perceive customer service, compliant handling, quality as well as environmental performance. These surveys are divided on a business area level, as well as on a country level.

Engagement in different networks is another source of input to PostNord's environmental work. PostNord is engaged in a variety of networks, such as NTM (a Swedish network for transports and environment). PN Logistics is also engaged in the work with Green corridors, which is managed by Trafikverket. In addition, PN Logistics is also involved in the KNEG network, (carbon neutral road transport), which was initiated by Schenker, the former Vägverket, Chalmers and Volvo Trucks. This is perceived as a good network forum and can be exploited to a greater extent since both fuel producers and vehicle manufacturers are gathered in order to develop together and show the need for a certain type of diesel across the country. 
- 130 - 


\section{PART III: CITY LOGISTICS - AN EXAMPLE OF A NEW GREEN SERVICE CONCEPT}


-132 - 


\subsection{Background to the city logistics concept}

When PN Logistics started to investigate the area of city logistics, it was stated that there was no common perception of this area. However, this view may depend on who is asked. The specification of requirements regarding this area is not clear, even though requirements exist from customers connected to certain parts within the city logistics area. However, these requirements are something that have been developed over time. Corresponding to the new strategies set recently, city logistics was identified as an area PostNord needed to take into account. This decision was partly based on the changing condition on the market regarding what customers demanded and also possibilities or risks connected to future changes in legislation or regulation within the urban areas.

PostNord wants to have a comprehensive picture of what city logistics means and the consequences for its business in advance of their competitors. Therefore, efforts are made to identify what city logistics is and how PostNord should act with regard to this concept in order to further develop its work within this area.

\subsubsection{Earlier experience regarding city logistics within PostNord}

Several business units within PostNord are, or have been, working with this field. For example, Mail Denmark has for some time worked and had contact with Copenhagen municipality and therefore has a clear picture of what city logistics can be involved in, within the service offerings. The business areas Mail Sweden and PN Logistics also have some experience regarding city logistics. A concrete example in Sweden is the city logistics project Norra Djurgårdsstaden, which represents a particular need within this area. The municipality of Stockholm invited PostNord to participate in this project, to act as a transport consultant and together with the other participants in the project try to create a city logistics solution. The Norra Djurgårdsstaden project was perceived by PostNord to be a good test object since it is a well-defined area and information exists regarding the number of inhabitants in the area, the number of households, the number of offices, the number of parking spots and so on.

Another example of a project that PostNord is involved in regarding different city logistics areas is the Cleantruck project, which includes geographical areas in both Stockholm and Gothenburg. PostNord participates in the project since they want to be a part of the city logistics area as well as to monitor its further development. The differences from previous initiatives within city logistics is that the focus of the city logistics field today is managed from a more comprehensive and structured approach.

The investments and efforts regarding the city logistics area are managed as a project within PostNord. Although there are several employees within PostNord working within this area, a project committee has indirectly been selected by the top management to be responsible for managing the projects and safeguarding the overall aim. The committee consists of employees from the Market and Sales department, production and those responsible for environmental issues, who represent the different business areas Mail and PN Logistics both in Sweden and Denmark. The work regarding this area has to date resulted in a number of workshops where different tasks have been divided among the employees in the committee and later been reconciled and followed up. These workshops can be seen as opportunities to share different kinds of information that is prepared and brought up by the committee members. For example, a number of customer surveys have been carried out within this area in Denmark. The survey results are discussed in these meetings and assessed to see if the findings are also 
relevant for the Swedish business. Another important aspect of this work is to create an acceptance in the organisation internally. There is an ongoing dialogue to reconcile the existing initiatives regarding city logistics in each of the different business units. The employees want to have a chance to impact what they are working with. If the committee succeeds with this establishment, the outcome of this work will be that everyone feels more actively involved in shaping the city logistics area.

As mentioned before, one of the reasons for trying to coordinate these types of workshops is to identify and collect all the knowledge and information within the organisation regarding this area, which can act as an input to continuing work for defining and shaping the city logistics area. In other words, this is done in order to use the benefits from previous experiences within this field. PN Logistics has been working with the Norra Djurgårdsstaden project for the past two years but has also received many requests from other municipalities in Sweden. When PostNord tried to reconcile the different specifications from the municipalities, a need to create an overall picture about this field internally within PostNord was identified. PostNord perceives a certain lack of knowledge of city logistics among the municipalities and as a result of this PostNord is expected to act as a consultant and solve their problems. This is not aligned with PostNord. The identification of how this should be done is one part in the proceeding work within the city logistics field. One of the reasons why PostNord wants to participate and influence the work of city logistics is the desire to be involved in the development of an industry standard regarding this area.

When it comes to the initiatives by PostNord's competitors within this area, efforts are made to map their development and how far they have come. Some competitors are perceived to be clearer than others when it comes to describing what they want to do and efforts actually performed within the city logistics area. Something that characterises these competitors, as well as other of PostNord's competitors, is the desire to be involved and push the development further. However, the clarity of delivering environmental solutions is not seen as particularly common within these discussions. Instead, PostNord wants to conduct operational efforts by documenting and creating a concept of how PostNord works within city logistics for each of their customers. This is something that is not done by their competitors today. This will be applied in the city logistics project in order to work towards a sustainable society. It is not just the matter of "green thinking" and carbon emissions issues; instead, it is about obtaining a holistic picture in order to create a sustainable environment for those people who are in that area. This approach is also consistent with PostNord's internal environmental goals. It is believed that city logistics is a pre-requisite for reaching these goals, for example by reducing the carbon emission by $40 \%$. Within the environmental area, continued efforts are being made to develop hybrid vehicles and replacements for electric vehicles. This is seen as a technical development that aims to support the overall vision of how PostNord wants to work. However, within the city logistics area, more efforts are put into the "operational toolbox" by groupage and shorter routes, in combination with the use of different types of environmentally-friendly cars. The focus should not solely concern reduction of carbon emissions; rather, the focus should be on looking at the big picture.

\subsubsection{Identified problems within the city logistics area}

Within PostNord, there is a perception that there is a certain amount of confusion among municipalities regarding procurements related to city logistics. This may be due to the fact that there are many different actors involved with different opinions on city logistics and its environmental impact. This may result in a contract that leads to a rather small impact on the 
environment or perhaps even a negative one. It is hard to point out a few essential points within city logistics where major environmental potential can be reached.

Instead, the city may be divided into different focus groups such as shops, restaurants, private consumers, warehouse locations and malls. This leads to different customer groups/categories with different requirements in the urban part of the city. In addition, when it comes to deciding which transport arrangements should be selected, this lies in the control of the receiver of the goods. However, it is usually a negotiating partner central in Sweden that decides in what way the goods should be delivered and the receiver has no influence over the transport arrangements that are to be selected. For example, a private consumer has more influence on the choice of an environmental alternative for how services bought online should be delivered than a shop keeper, where the decision on the deliveries may be determined elsewhere. This central body may decide how the deliveries to the stores should be made and the decision may be based more on price than on other factors that might affect the deliveries. The contracting party may not take into account whether there are environmental zones in certain parts of the city or a specific time schedule for delivery.

Thus, there are forces that work against each other and complicate the suggested solutions related to city logistics. There are many different actors involved, and there is a need to find a viable commercial solution within the city logistics area. If an actor had seen an economic potential within this area the development would have come a lot further, compared to where it stands today. As long as PostNord can deliver the goods to their customers, not much attention is paid to how this will be conducted. The problems with city logistics are perceived to concern partly the environment, congestion or noise issues in a city, which is not directly perceived as a problem to be solved by shippers. It should perhaps lie in the interest of municipalities to react to this problem area and find a solution. However, the constant question is:

"What is the problem to be solved and who owns the problem?" (Environmental Manager, PN Logistics, 11 October 2012)

The municipalities are not really perceived as taking responsibility regarding these kinds of matters. There is a need to take responsibility for both traffic policy issues as well as various types of development issues in order to create rules for how the city will develop. For example, within the Norra Djurgårdsstaden city logistics project, there are no plans for how the goods to the different malls should be delivered or how the return flows of waste should be configured. Based on this project, PostNord aims to define a generic roadmap for city logistics by developing a methodology and a process description with related problem issues and toolkits. This approach is intended to be embedded in both local municipalities as well as centrally within PostNord. If such an approach can be found together with an approach to how this can be communicated, then PostNord will have come a long way in the development of the city logistics area.

\subsubsection{The next step within the city logistics concept}

As mentioned before, the efforts made by identifying and collecting information, together with earlier experiences and knowledge within PostNord regarding city logistics acts as an input to develop a plan. This plan is realised in order to support the decision of whether or not PostNord should continue within this field. This plan will be presented to the top management team at the end of 2012. The decision support should involve: 
- A definition of city logistics;

- Map and identify initiatives taken by the different business units within this area, in order to obtain a complete picture of city logistics;

- Suggest a potential approach for the ongoing work; an "all in" approach or testing in a smaller extent.

In addition, a "goal picture" of city logistics will also be included in the plan, where, for example, environmental targets will be one area. Examples of other areas that may be included in the "goal picture" are:

- Goals or targets regarding carbon emissions. The aim is not to develop different kinds of goal levels. Instead, based on the overall environmental goal of PostNord, discussions will be held with customers regarding what these goals mean for them.

- Presentation of added values. Focus should not only include carbon emissions but what this actually means and how PostNord can promote it. A description including what and how this can be communicated with the customers.

- A link to profitability and growth opportunities. When it comes to growth opportunities, there is already an assessment of the potential possibilities with the city logistics market and also estimations regarding what rate and how many percentages of the market will be reached.

- Potential solutions or service offerings. This area is not fully developed but can be seen from different time perspectives.

$\circ$ From a shorter perspective. This may involve identification of what can be done within PostNord today; which objectives should be tested and how should the evaluation of these tests be done?

o From a longer perspective. Identification of an existing or potential need in order to develop solutions to the city logistics area. This may perhaps involve some part of the vehicle fleet or be linked to the system support both for internal coordination, but also to support a new kind of customer interface. If PostNord takes the decision to invest in the city logistics area, there will be a need to work with these kinds of issues. Nevertheless, when it comes to investments in the vehicle fleet, each business unit will be responsible for taking this development further.

- Identify potential collaboration partners. Both from an operational and a research and quality perspective.

Regarding the definition of city logistics, this formulation cannot be too narrow or designed for a specific city or municipality. Instead, PostNord will need to have a fairly broad approach and "toolbox" in order to solve problems for different types of sustainable commercial urban areas. In the future, there may be a developed city logistics concept with some kind of product description and underlying adding services. However, PostNord will not wait until it has all the answers. Instead the company intends to start testing different scenarios and thereafter start the development of service offerings.

"This is seen as a "test bench", you have to start somewhere." (Senior Project Manager, PostNord, 11 October 2012)

A current step within the city logistics area is to set the stage and create conditions for this area. Based on this, each business area can thereafter continue to work on different initiatives 
in order to realise the set strategies regarding city logistics. PN Logistics will manage the main share of the responsibility for the city logistics area, even though there is a desire for a more cross-functional collaboration between the different business areas. The city logistics area is an example of an initiative whose boundaries span over different business areas, as well as between Sweden and Denmark. However, PostNord does not want to have a separate city logistics concept in Denmark and Sweden.

From an overall perspective, different kinds of city logistics projects exist and have been executed; however, PostNord has not recognised any other transport operator with a similar approach to PostNord regarding city logistics. At the same time as the main city logistics project has been driven by the committee, initiatives have been taken by PN Logistics to start a network for city logistics. In addition to employees from different parts of PostNord, the network also consists of representatives from Linköping University, VTI and SKL, as well as a municipality in Sweden. The aim of the network is to find further information and knowledge of the city logistics area and together with the experience within PostNord create some kind of consensus. The proposal to create a network is seen as a good example of an initiative taken by a business area in order to be involved and also to be able to influence the further process regarding the city logistics area within PostNord. 
$-138-$ 


\section{CHAPTER}

\section{ANALYSIS - TOWARDS LSPS' GREEN SERVICE DEVELOPMENT}

This analysis chapter consists of three main parts. Firstly, the analysis takes a stance from the analysis model presented in Chapter 3, and analyses the identified NSD dimensions separately in order to identify underlying pre-requisites as well as their role in the context of LSPs' general NSD efforts. Secondly, the identified NSD dimensions' influences and links to each other are discussed, resulting in an NSD framework for LSPs. Thirdly, the analysis builds on the second part and analyses the different NSD dimensions from a green service development perspective. In order to give the reader an insight into the main parts and their connection to the research questions, a short presentation of the analysis approach is presented at the beginning of this chapter. 
-140 - 


\subsection{The analysis approach}

The analysis model developed in Chapter 3 together with the different research questions of this dissertation, form the base as well as the structure of this analysis. The analysis is divided into three different sections and follows a step-wise approach where the outcome from each section is further developed in the next section. The connection between the different analysis sections and the research questions is illustrated in Figure 7.1 below.

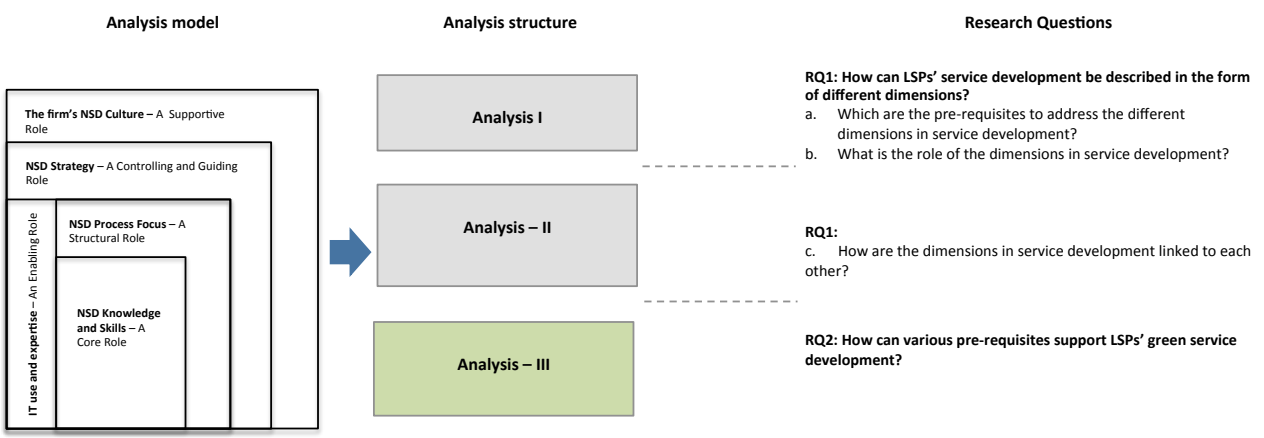

Figure 7.1 The analysis approach.

\subsection{Analysis I-Identifying pre-requisites for \& roles of the NSD dimensions}

The first section of the analysis addresses RQ1a-b as illustrated in Figure 7.1. As a first step, the different NSD dimensions in the analysis model will be analysed separately. Each dimension takes a stance in the literature findings regarding LSPs' NSD (as described in Chapter 4), and the discussion is influenced by the literature findings from the general service development perspective in Chapter 3. In addition, the PostNord case study provides empirical input to the analysis in the form descriptions as well as illustrative examples. The goal of this analysis step is to identify and concretise pre-requisites for each of the different NSD dimensions. In addition, each NSD dimension ends with a summarisation clarifying the overall role of the identified dimension and its pre-requisites for an LSP's service development management.

\subsubsection{Creating an NSD Culture}

Both the NSD and the LSP literature emphasise the existence of an NSD culture that provides a climate that facilitates and fosters NSD activities as necessary and essential in order to achieve NSD success (e.g. Menor \& Roth, 2007; Jaw et al., 2010; Busse \& Wagner, 2008b). For example, Flint et al. (2005) suggest that setting the stage for an LSP's NSD activities involves creation of a creative and supportive environment for employees as well as involved partners. In addition, Busse and Wagner (2008a) mention some success factors for creating such a culture among LSPs such as a central coordination centre, hierarchical control of innovations project and existence of interdisciplinary team structure. However, the form of such management systems, as mentioned in the NSD literature, are manifested in the company's development structure, corporate culture, communications norms as well as top management support. 
The LSP industry has earlier been rather production-oriented in its operations but has through increased strategic focus started to become more customer-oriented by providing more customised logistics solutions for its customers. By changing focus it may be relevant to argue that it will have an impact on an LSP's NSD culture and activities.

This can be exemplified in the case study regarding the culture between the different operational units of PN Logistics. The management of and responsibility for the service development differs between the operational units in PN Logistics. The operational units in Sweden, Denmark and Finland have a special unit for service development, while in Norway it is more integrated in the overall business activities. However, PN Logistics strives to have a more uniform structure of service development within the different countries. Discussions are held through forums on how activities can be better coordinated between the different countries. These discussions also concern sales activities, e.g. development of a common CRM strategy, partnerships with customers as well as business development with customers. In Sweden and Denmark, the culture has been more influenced by structural processes and stringent requirements to achieve the goals set, while in Norway there is a stronger customerfocused climate, which affects the control of the development, organisation and management priorities as well as influences the culture and attitude. Despite the different organisational cultures, PN Logistics can identify advantages when these two cultures meet:

\section{"This can allow initiatives to be taken at an earlier stage and to dare to test in a small scale, but at the same time should be rely on a certain structure."}

Having a more customer-oriented approach to NSD and ensuring customers' input in the NSD process is also mentioned in the NSD literature as important due to customers' changing needs and expectations (e.g. Jaw et al., 2010; Michel et al., 2008). This trend regarding changing customer behaviour can also be found within the LSP industry, which forces LSPs to move closer to the market expectations but also to consider how customers can be integrated more in their NSD efforts. Due to the intangibility of logistics service offerings, a more customer-oriented approach can help LSPs to ensure the quality of the service offerings provided. In addition, it can also help to overcome some barriers, such as difficulties of testing new concepts as mentioned in the LSP literature as well as in the case study. The above discussion leads to the first pre-requisite regarding an LSP's NSD culture:

- Create a customer-oriented NSD culture aligned with the organisational structure and processes

In addition, in order to create an NSD culture that supports and increases employees' knowledge regarding NSD activities, it must also promote a learning approach regarding both previous and new NSD knowledge and skills within the company. As mentioned in both the NSD and the LSP literature, utilisation of cross-functional teams can be a way to achieve this learning approach (e.g. Froehle \& Roth, 2007; de Jong \& Vermuelen, 2003; Flint et al., 2008). Due to the evolving trend of logistics service offerings becoming more complex and information-intensive, the existence and attitude towards cross-functional teams may be of essential importance for LSPs when developing services. Promoting a cross-functional approach may not only benefit the clue-gathering phase but also add value to the entire development process of logistics services. As referred to in the LSP literature by Panayides (2007), managers should stress the value of learning and facilitate this by encouraging employees to work together in achieving common goals, sharing information within their own unit as well as with others and sharing lessons learned during the NSD process. Having a 
more positive approach towards learning may also lead to identification of "best practice" of NSD activities within the company.

As mentioned in the case study, the approach the operational unit in Norway is working with identifying customer needs and strengthening the relationships is considered within PN Logistics as successful. Therefore, this approach is to be integrated within the business area in Sweden as well as the other countries. It represents a good example of best practice sharing and generates learning and knowledge about the customers.

The LSP industry is characterised by a low integration of external partners in their NSD efforts and Kuester et al. (2013) argue that this requires a strong focus on internal cooperation in order to be effective and successful in their NSD efforts. However, the importance of internal cooperation and intra-organisational learning may increase in the future due to changing customer behaviour and requirements. It may also be influenced by the growing internationalisation trend towards more complex and cross-border logistics solutions.

The need for internal cooperation connected to customers' new and changing demands is mentioned in the case study. For example, within the e-commerce business, the business units Mail and PN Logistics have identified similar customer needs, and efforts have been made to bundle different service offerings to this customer segment. By exchanging experiences in the e-commerce field, such collaboration initiative may also prevent the risk of overlapping service offerings. This may lead to the best utilisation of the resources available, and also prevents the risk of double work.

Hence, in order to meet the changing market environment and improve efficiency of the resource base, LSPs may strive for a more learning-focused approach in order to identify synergies as well as "best practice" both within and between the different business units. For example, as mentioned above, PN Logistics uses different forums in order to discuss how activities in the different operational units can be better coordinated. This concerns not only service development but also sales activities, such as the development of a common CRM strategy, partnerships, as well as business development with customers.

Therefore, based on the above discussion, LSPs need to put focus and efforts into creating pre-requisites concerning:

\section{- Emphasise intra-organisational learning of NSD for increased resource efficiency}

Furthermore, an NSD culture should reflect not only a company's internal approach towards working with NSD activities but also an external perspective. The LSP industry has in the past been fairly production-oriented but through increased strategic focus, it has started to become more customer-oriented by serving all logistics needs for customers (Chapman et al., 2003). As mentioned in the NSD literature, adopting a more customer-oriented approach may be necessary in order to quickly adjust and respond to customers' changing needs and expectations. However, this places requirements on the NSD as well as on LSPs' ability to gain information and understand their customers' values and needs. Thus, in order to facilitate understanding and sharing of knowledge, as well as ensure replication of lessons learned, LSPs should strive towards a more learning approach with their customers as well. In addition, LSPs may expand the boundaries and include other external partners, e.g. suppliers, into a more learning approach when developing new services, since, as mentioned in the NSD literature (e.g. Michel et al., 2008; Schleimer \& Schulman, 2011), collaboration with different 
external partners can result in sources of new knowledge and operant resources that lead to development of superior service offerings.

An example of having a more external learning approach towards NSD is mentioned in the case study concerning PN Logistics work with reverse logistics. New kinds of customer needs have started to emerge within this area, and PN Logistics uses different actors as an input in order to meet these demands. Dialogues and collaboration efforts with involved partners and other actors such as developers of TA systems have been conducted. In addition, specific customers have also been invited to participate and PN Logistics has also made reference visits at the customers' companies. The information from the different actors resulted through internal discussions in a number of service development initiatives within this area.

Having a more inter-organisational approach or, as Flint et al. (2008) refer to it, a "supply chain management learning" approach for its NSD efforts, may lead to better dialogues and finding better solutions for serving each other in the supply chain. This may be of specific relevance for LSPs when developing more solution-oriented service offerings for customers, which not only requires knowledge and information regarding customers but also the customers' supply chain. On the other hand, some LSPs may provide more basic transportation service offerings, which requires less inter-organisational learning since the service offerings may be less complex. This is in line with Syson and Perks (2004), who argue that the range of actors involved, combinations of resources and activities undertaken depend on the level of newness of the service developed. The above discussion leads thus to the following pre-requisite regarding LSPs' NSD culture;

\section{- Create a culture that promotes inter-organisational learning in line with the strategic NSD intent}

In sum, the existence of a culture that fosters and shows awareness of NSD is seen as an important element for LSPs' NSD (Busse \& Wagner, 2008b). The role of an LSP's NSD culture is to provide a basis for the company's ability and attitude to develop new services. Or as Flint et al. (2005) describe it: to set the stage and thus act as a foundation for managing and supporting an LSP's NSD activities. Like many other service companies, LSPs' key resources for NSD consist of their employees. As a consequence, an NSD culture among LSPs should strive to take advantage of employees' knowledge and skills and promote information sharing and cross-functional efforts when developing new services. However, as suggested in the above analysis, an LSP's NSD culture should not solely consider an internal focus but also create an NSD culture that promotes and supports learning from other actors. Thus, the NSD culture should not completely be viewed as something static, but rather, as suggested by Flint et al. (2005), this foundation should be modified and refined over time based upon previous learning of an LSP's NSD activities. It is worth mentioning, that in order to realise such an NSD culture many researchers stress the relevance of top management as well as managerial engagement and support (e.g. Busse \& Wagner, 2008b; Panayides, 2007; Flint et al., 2005). In addition, since the LSP industry is described as having "transformed from the traditional business notion of simple point-to-point transportation to that of serving the entire suite of customers' logistical needs" (de Souza \& Goh, 2008, p. 62), this places requirements on LSPs to better understand and respond to customers' demands. Therefore, LSPs may benefit from integrating a more customer-oriented approach in their NSD culture. 


\subsubsection{A strategic focus of NSD and existence of an NSD strategy}

Within the NSD literature, an NSD strategy is described as a plan or strategic platform that sets the conditions as well as facilitates the management and availability of relevant resources for NSD efforts (e.g. Menor \& Roth, 2008; Kuczmarski \& Mandolia, 2012). Within the LSP literature, little focus has been put on these actors' NSD strategy even if NSD has been identified and suggested as a way to increase LSPs' further growth (Busse \& Wallenburg, 2011). Thus, it may be reasonable to argue that almost every LSP or any other company has, to some degree, a strategic focus and strategies for its service development efforts. The differences between companies' NSD strategies concern the extent and the elements included in the scope of the strategic focus of NSD.

As a first step to defining an NSD strategy, it is relevant to clarify the service vision and the strategic role and relevance NSD plays for a company. Within the LSP literature, Oke (2008) reveals that a clear definition of NSD and what it actually means for the company seems to be lacking among LSPs. However, the author argues at the same time that defining the strategic role of NSD can help LSPs to focus on the most appropriate NSD areas for the company.

Defining the strategic orientation of NSD can also be influenced by differentiation approaches (Busse \& Wagner, 2008b) since it can be a way to distinguish an LSP from its competitors (e.g. Flint et al., 2005; Wagner, 2008) but also increase the trustworthiness (Wallenburg, 2009). In addition, how the LSP chooses to position itself on the market can be a way to help the LSP to create appropriate conditions for NSD and match innovation efforts to the strategic choices of the company (Flowers et al., 2008).

In addition, another aspect mentioned in the NSD literature that may influence the definition of the strategic role of NSD concerns the selection of target markets. This may be of special relevance for many LSPs since the LSP industry is no longer seen as an emerging market, which thus puts focus on taking market shares from competitors (Wagner \& Franklin, 2008). Examples of different incentives mentioned in the literature for LSPs' service development can be, for example, increasing loyalty of existing customers or attracting new ones, which market to focus on, or the need to introduce new services in order to create or maintain a dominant position on existing market. According to Wallenburg (2009), the LSPs' main incentives for service development concern mainly improved service quality, reduced cost and improved existing customer relationships.

As mentioned in the case study, PN Logistics wants to continue to grow with existing customers. There is a desire not to be perceived as a subcontractor; instead, PN Logistics wants to develop closer relationships with its customers and be regarded as a partner. In addition, through the "Nordic products" concept, PN Logistics wants to be transnational in order to provide comprehensive logistics service offerings in the Nordic region. The reason for the "Nordic products" concept is explained by PN Logistics as follows:

"It is very much about improving the already existing service offering and adding customer value by combining different additional services and becoming more transparent about, for example, environmental data. This approach will also be easier to understand from a customer perspective."

Based on the above discussion, it may be relevant to argue that LSPs need to identify the main incentives for their NSD ambitions as well as define their strategic role in order keep a focus on the NSD efforts. This leads to the first pre-requisite for LSPs regarding NSD strategy: 
After determining the strategic role of the LSP's NSD efforts, these must be formalised into clear and defined goals regarding, for example, financial and growth aspects in the NSD strategy. Both the NSD and the LSP literature stress the importance of the alignment between the NSD strategy and overall business strategy. The NSD strategy developed should also support and connect with other functional strategies within the company (Busse \& Wagner, 2008b); however, this aspect was not explicitly mentioned in the NSD literature.

According to PN Logistics, their NSD strategy is fully aligned with the overall service strategy, which has a more controlling role. Other examples of strategies that support the NSD strategy as well as the service strategy concern the production and sales area. For example, the production strategy includes the entire PostNord business area and provides opportunities, as well as limitations, for the NSD strategy. This exemplifies the relevance of a defined NSD strategy in order to understand how it can support and contribute to other strategies, but also how it can be affected and adapted by other controlling strategies in the company.

Besides an alignment with the overall business strategy, the NSD literature also mentions that the NSD strategy should fit the company image. This was also mentioned within the case study as a strategically important element in delivering the service strategy. PN Logistics emphasises that:

"It is important to have a living brand and, in order to facilitate the communication, how should we describe ourselves, what industries should we focus on and grow within and how can we try to become partner aligned with customers?"

A defined NSD strategy does not only help LSPs to stay focused on their NSD efforts. Through its alignment with other strategies and the company's image it can also support the achievement of determined strategic goals as well as facilitate both internal and external communication. This, in turn, initiates another pre-requisite regarding LSPs' NSD strategy:

\section{- Develop an NSD strategy that fits the overall strategic goals}

Furthermore, as mentioned in the NSD literature, the NSD strategy needs to include an external link with customers' expectations and demands. This is also supported in the case study, where market analysis regarding, for example, customer requirements, competitor development as well as environmental and technical regulations on the market, act as an input to the service strategy. As mentioned in the case study, one strategy stated within PostNord is to continue to grow together with existing customers. In order to gather insight to this work, a number of key customers have been identified in order to find potential solutions that go beyond the boundaries of the different business areas. Corresponding activities with key customers are also conducted within the PN Logistics business area. These customers are selected due to the fact that they have operations in many countries, since this is an area within which PN Logistics believes it has potential to grow.

After the discussion in section 7.2.1 regarding LSPs becoming more customer-oriented, it may be of particular importance for LSPs to consider this element. In order to develop new services and meet customer needs, Speh (2008) argues for a deeper marketing understanding among LSPs. There is a need to match the selected target market with the marketing mix 
(service product, pricing, promotion and distribution) in order to identify the expectations of each segment.

Thus, identifying different customer demands as well as other external factors may not be enough. To fully benefit from the NSD efforts and develop appropriate service offerings, LSPs will need to take one step further and select and match relevant customers' demands to their NSD strategy. This is relevant both from a short and long-term perspective. This leads to the pre-requisite:

\section{- Segmenting and matching customers' demands to the strategic NSD focus}

According to the NSD literature regarding internal focus of the NSD strategy, the NSD strategy should confirm an internal strategic alignment of appropriated resources and practices of the developing company. This can be a way for a company to identify if the current resources are sufficient, or if there is a need for adjustment or modification of the existing facilities. PostNord made several investments during 2012 in order to improve opportunities for additional growth and provide services and end-to-end solutions that better match customer demands. For example, some major acquisitions were made in order to enhance strength in selected submarkets and expand the geographic coverage. Investments were also made regarding establishment of a new combined parcel and pallet terminal and related to vehicles in order to streamline production efforts and create possibilities for capacity expansion. This was not done for any specific customer; instead, these investments in resources have a more long-term and strategic focus. Other strategic resources mentioned both in the LSP literature and the case study concern IT and technology investments. However, it may not be possible to create a comprehensive resource base supporting all kinds of NSD efforts, as illustrated in the case study:

"...you always have to make some priorities when it comes to internal resources as well as IT development resources."

However, as can be found in the annual study of Third-Party Logistics (2013), customers perceive LSPs' IT investments as mainstream and conservative and their IT capabilities as rather low. This raises a general requirement for LSPs to evaluate their IT investments in order to ensure that it not only supports their business but also matches with market expectations. In addition, for the specific aim of this dissertation, how it can support LSPs' NSD efforts. Within the NSD literature, IT investments are said to increase generation of new ideas, accelerate and support the development of new services and overall improve the rapidness of new services in reaching the market.

However, PN Logistics perceives that it is often a matter of making priorities when it comes to IT's supportive role in relation to service development. Another challenge for PN Logistics concerns the outsourcing of some part of the operation and development of IT, which has resulted in a perception of getting less for the money and also that it takes longer. In order to ensure IT support for NSD efforts, LSPs may need to define its strategic relevance as a component in the design and production of their service offerings, identify IT systems and tools supporting this development and match these to the planned IT investments.

Thus, it is essential for LSPs to identify the appropriate strategic resources supporting their current NSD efforts but also investments of resources from a more long-term and proactive 
perspective in order to meet customers' demands in the future. Thus LSPs need to consider the following pre-requisite:

- Match IT to its strategic NSD importance to the same extent as other internal resources investments

Both the NSD literature and the LSP literature stress the relevance of aligning the NSD strategy to the company's business units. In addition, Busse and Wagner (2008b) argue that the content of the NSD strategy needs thus be further concretised and specified in order to match a specific business unit within the LSPs. This can be supported in the case study, each of PN Logistics' operational units in the different countries has its own unique strategy underlying the overall service strategy of PN Logistics. For each operational unit, a specific target regarding the service development, e.g. sales goals, is set. The match between the NSD strategy and an LSP's business units may be necessary since many large LSPs have a broad range of services in their product portfolio and operate in different markets. The different business units may therefore face different market conditions in the form of customers' requirements and preferences, have different resource requirements in relation to their service development efforts and also aim for different market positions. Therefore, in order to create the appropriate NSD conditions as well as set NSD targets for each business unit, LSPs need to focus on the pre-requisite regarding:

\section{- Alignment and concretisation of the NSD strategy to the different business context}

Another important aspect mentioned both in the NSD and LSP literature concerns the link between the NSD strategy and the content of existing service offerings. LSPs need to understand the options for expanding their business scope (Sonio et al., 2012). According to Shen et al. (2009), LSPs' strategic NSD today focuses on modularisation and reduction of logistics cost but stresses at the same time the trend towards more customer-oriented service offerings.

This is supported in the case study, where PN Logistics strives, through its "Nordic products" concept to create a range of different basic services with a variety of additional services in order to combine these with specific customer needs. The goal is to offer a customised solution by using existing building blocks in order to gain efficiency in their own production mechanisms. Having too broad a variety of service offerings in the product portfolio as mentioned in both the case study and the literature reviews can result in high costs, overlapping services and an unclear picture of the service offerings to customers. This forces LSPs to reconsider phasing out certain service offerings and focus on the strategic focus and alignment with existing service offerings. This leads to the following pre-requisite:

\section{- Ensuring fit between new service offerings and existing resources}

To conclude, the above discussion reveals some fundamental aspects for LSPs when developing an NSD strategy. These have been summarised into pre-requisites valuable for LSPs to consider regarding their NSD efforts. The role of an NSD strategy is often mentioned as a key factor in improving NSD performance. Within the LSP literature, the NSD strategy is viewed to be the centre position among all the different activities related to NSD among LSPs (Zhou \& Wang, 2012). Moreover, as mentioned in the LSP literature, those LSPs that make strategic NSD choices in relation to their external environment and internal resources, as well as understand the conditions for expanding their service scope, will be those actors that 
achieve growth and push the development of the LSP industry further (e.g. Zhou \& Wang, 2012; Speh, 2008). Based on this reasoning, the NSD strategy can be described as the centre of LSPs' NSD efforts. Through the NSD strategy's defined approaches and goals, it sets the parameters of the LSPs' NSD focus areas as well as aligns internal resources, which in turn gives the NSD strategy a controlling role in LSPs' NSD efforts. In addition, by matching the NSD strategy to LSPs' surrounding business environment as well as customers' needs and requirements, the NSD strategy receives a more competitive important role for LSPs' further development.

\subsubsection{A focus on processes and structural relevance of NSD}

Within the LSP literature, it is suggested that LSPs do not employ formal NSD process due to the fact that NSD is a response to customers' requests and also that these processes are viewed as too time-consuming (Franklin, 2008). However, both the NSD and LSP literature advocate that an NSD process is necessary for conducting the NSD efforts. The question to ask is then: what pre-requisites should LSPs consider regarding management of the NSD process to fully benefit from its NSD efforts?

The formalisation of the NSD process and its included steps can be seen as a start, since it has been proven to support NSD among LSPs rather than prevent it (Daugerthy et al., 2011). The LSP's NSD process is suggested to obligatory structuring the flow and decision making (Busse \& Wagner, 2008a) and by its existence provides both accountability and consistency regarding the service offering provided to the customers (Oke, 2008). As mentioned in the NSD literature, each step in the NSD process provides an input and has a function for the entire NSD process. However, some steps are regarded as more vital than others, for example idea generation and screening and selecting NSD ideas. These steps were found to be more challenging than the others steps among the LSPs in the study by Busse and Wagner (2008a).

This can also be supported in the PN Logistics case, where these steps (idea generation and screening and selecting NSD ideas) require quick decision-making, good knowledge of the company's strategic NSD focus, as well as valuable input from market and customer surveys. This in turn reveals the relevance of creating formal practices and routines as a support for employees in the NSD process. Thus, this applies to all steps in the NSD process. PN Logistics has a set plan and structure for how the general service development process should proceed. It consists of three main processes: develop services, sell services and produce services. It supports developments of both existing and new services. In addition, there is an ambition to implement the process model for developing services in the rest of the operational units. A lack of appropriate NSD processes and routines may make it more difficult for LSPs to capture return from their NSD efforts (Wagner \& Franklin, 2008) as well as complicate the management of it.

In addition, as mentioned in both the NSD and the LSP literature, there is also a need to find a flexible structure for managing the NSD process. LSPs should emphasise the fact that different NSD initiatives may require different amounts of resources and levels of formalisation. The customisation of the NSD process, as mentioned in the NSD literature, can depend on factors such as the characteristics of the particular target market, the newness of the service or time to market (e.g. Johnson et al., 2000; Cowell, 1988). The characteristic time to market was especially highlighted in the PN Logistics case study as an important factor regarding the NSD process. The need to customise the NSD process to different NSD projects can further be illustrated in the case study: 
“..we are extremely structured, sometimes maybe a little too structured when it comes to how to run minor changes. Especially if the change is connected to IT, then the process becomes very structured. It can involve, for example, who makes decisions, what kind of order is it, how should the testing be approached, who will accept delivery and so on."

The discussion above reveals the complexity of the NSD process. A high level of structure requires a great deal of resources and is perceived to be very time consuming. This promotes the relevance of finding a balance between a highly formalised NSD process and a more ad hoc approach. This calls for a need for LSPs to consider the pre-requisite:

- Allow for a flexible and customised management within the structure of the NSD process

However, allowing for a certain degree of a more structured and formalised NSD process can facilitate the involvement of different employees and their understanding of the included steps and activities. Both within the NSD and the LSP literature, involving cross-functional efforts in the NSD process is seen as a critical factor. This may be a relevant factor for LSPs to consider when moving forward to more solution-oriented service offerings, which will most likely require various forms of knowledge and input from the company's different functional units.

This can be supported in the case study, where the composition and involvement of crossfunctional teams within the NSD process are seen as central for the future work. By working in teams, PN Logistics tries to use the information from the market analysis as effectively as possible. A variety of competences are present within these teams, ranging from skills regarding service development, marketing and communications at a Nordic level. The Vice President of Business Development, PN Logistics states that:

"This is seen as a huge strength as well as the importance of the informal communication between the people involved in these teams."

In addition, a need to further increase cooperation between functional units in order to identify and integrate new knowledge and input to the NSD process has also been identified within the operational unit in Sweden.

Further, the customers' perceived value of the new service offering will, according to Flint $e t$ al. (2008), depend on how formal cross-functional and cross-organisational management of the NSD process are handled. This is in line with Chapman et al. (2003), who recommend that LSPs gain and integrate new knowledge to the NSD process by considering the full extent and breadth of their functions, systems and processes in the whole supply chain. For example, customers can be one example of external actors, which can bring new insights and knowledge into the NSD process. As mentioned in the case study, PN Logistics argues that customers must somehow be involved all the way through the NSD process. During the test phase of an NSD project, customer participation is seen as a necessity in order to obtain input and predict possible outcomes of the project.

However, in addition to customers, there may be other external actors or networks relevant for LSPs to integrate and coordinate into the NSD process. The gaining of new knowledge and especially how this can be integrated and managed in the NSD process may become of essential importance for LSPs, due to the future development of more industry-specific 
solutions within the LSP industry. This discussion therefore results in the following prerequisite regarding:

- Integrate and coordinate cross-functional and cross-organisational knowledge in the NSD process

Other positive effects of structuring and formalising the NSD process mentioned in the NSD literature concern the company's ability to repeat or improve included steps or activities. The existence of feedback loops in the NSD process is seen as critical and can facilitate analysing the intangible aspects of the service design (Johnson et al., 2000). This aspect is also mentioned in LSP literature, for example existence of a feedback culture, dedicated review time (Busse \& Wagner, 2008a) as well as a need for LSPs to increase their ability to capture repeating benefits from their NSD efforts (Pekkarinen \& Ulkuniemi, 2008). This raises a need for LSPs not only to focus on the evaluation of the new service offerings in the form of relevant economic measures and customers perception derived from various market research, but also to focus on the lessons learned from the different NSD projects. Thus, there may be a need for LSPs to establish formal procedures for gathering lessons learned from specific NSD project as well as ensuring replication of successful NSD efforts. This may be of particular importance, through an increased integration and collaboration of external partners in the NSD process. For those reasons, LSPs need to develop pre-requisites concerning:

\section{- Create formal procedures for evaluation and replication of NSD efforts}

As mentioned in the LSP literature, many LSPs strive to become more customer-oriented and try to modify or customise their service offerings in order to meet unique requirements from their customers. For example, Wagner and Franklin (2008) illustrate in their model that using common service platforms combined with specific standard tools will facilitate the management for LSPs to rapidly develop customised service offerings or solutions to specific customers. In addition, due to its level of standardisation this leads to opportunities for LSPs to reuse these kinds of service offerings on a greater scale.

As mentioned in the PN Logistics case study, there is overall pressure within the company to reduce the time to market by finding smart, effective and low-cost routines and solutions. In addition, in line with the reasoning of Wagner and Franklin (2008), PN Logistics has, through its Nordic products, developed a "model building" approach in order to quickly customise and broaden the range of service offerings to customers. Using existing building blocks in their own production mechanism leads to efficiency and allows a certain level of standardisation. This approach is also seen as quality assurance, since a broad variety of special solutions can increase the risk of mistakes in the process due to the number of people who are involved in the delivery process of the service. The quality assurance aspect is also highlighted by Wagner and Franklin (2008), who further stress that a more "platform thinking" approach has a positive effect on the developed service's reliability. In addition, Cui et al. (2009) stress other benefits of using a "platform thinking" approach when providing customised service offerings, such as successful differentiation in the market, increased customer loyalty as well as gaining new customers. However, extending the customised solutions to a broader customer base is seen as a challenge by many LSPs but may be necessary due to the LSP industry's strong focus on economies of scale. There is a need for LSPs to put efforts into finding ways to reuse knowledge and resources dedicated for a specific customer to a broader customer base. The above reasoning leads to the following pre-requisite: 
To sum up, according to the LSP literature, there appears to be a scarcity of structured NSD process among LSPs. However, the above discussion has attempted to prove its importance, which has resulted in the identification of pre-requisites regarding NSD process that may be relevant and necessary for LSPs to consider when developing new services. Regarding the overall role of LSPs' NSD processes, it can be argued that the existence of a certain structure or formalisation of NSD processes can be seen as a fundamental structure. This structure needs to exist in order to enable and facilitate the management, evaluation, learning and improvement of its included steps and activities. Based on the above discussion, the NSD process of LSPs can be seen to have a more coordinating role both regarding internal NSD activities and cross-functional collaboration but also concerning external actors' involvement in the NSD process. Compared to other service providers, the external focus may be of special relevance for LSPs since they have not only to interact with their customers but also with the customers' customers and suppliers. Furthermore, due to the intangibility of logistics services, LSPs' NSD processes may also, both internally and externally, hold the role of a certain achieved quality guarantee and thus ensure the reliability of the developed service offering.

\subsubsection{The IT adoption and expertise regarding NSD}

As mentioned in the NSD literature, the adoption of an IT system provides a range of possibilities to support NSD activities and is regarded as one of the most important infrastructural elements for many service companies. As mentioned in the LSP literature, this evolving ICT trend has also reached the LSP industry and has affected both their business and service offerings. It is seen as an important part in LSPs' service development efforts (Zhou \& Wang, 2012) but also as a key aspect and opportunity to further drive new service initiatives among these actors (Third-Party Logistics Study, 2013). Thus, LSPs need to ask themselves what kind of pre-requisites are needed in order to fully take advantage of their IT systems and tools concerning their NSD efforts.

One aspect mentioned in the LSP literature, which is needed in order to fully take advantage of IT benefits, concerns the integration and synchronisation of IT systems. This aspect can be relevant for LSPs both from an internal and external perspective. For example, synchronisation of IT systems can result in a more streamlined management process as well as efficiency and productivity improvements across the supply chain (Chapman et al., 2003). As illustrated in Table 13 (see section 5.2) regarding LSPs' ICT integration with other actors in the supply chain, integration with customers as well as other LSPs seems to be the most common aspect. However, in general the results reveal relatively low means, which might reflect the limited extent of LSPs' ICT integration with other actors in the supply chain. Despite this, there seems to be a rather large interest among customers in a more strategic technical relationship with their LSPs (Third-Party Logistics Study, 2013). By integrating with customers into different IT solutions, it is suggested that LSPs might gain valuable technology-specific knowledge as well as increased expertise within this area (Wagner \& Sutter, 2012). Moreover, as mentioned in the PN Logsitics case, building an electronically connection with customers is seen as a way to strengthen the relationships with its customers.

LSPs can also benefit from IT synchronisation efforts from an internal perspective. As mentioned in the NSD literature, maintaining supportive back-office IT systems and ensuring their compatibility is important in the production and delivery process of NSD (Froehle \& Roth, 2007). This is of particular relevance for information-intensive services, which logistics services have increasingly tended to become (Third-Party Logistics Study, 2013). This can be 
supported in the PN Logistics case study, from having a very fragmented portfolio of systems within PostNord, investments have been made in building a common IT system for all the business areas in all the different countries. This has, for example, been done with their Track and Trace solution, with the aim of providing the same type of information in all the different countries. In addition, synchronising and streamlining IT systems within the company may provide similar IT conditions for the different business areas within LSPs and facilitate increased NSD collaboration not only between business areas but also between operational units in different countries. The above discussion suggests that in order for LSPs to further benefit from their NSD IT efforts, LSPs need to focus on the following pre-requisite to:

\section{- Enable synchronisation of IT systems both internal and external}

In relation to the above-mentioned pre-requisite, the next aspect to be discussed concerns IT as a way to improve information sharing and communication. As mentioned in the NSD literature, IT can act as a facilitator for the systematic gathering and processing of large amounts of information, which in turn can be used as an input to NSD activities. However, the availability of this data and information will depend on an LSP's ability to organise and disseminate this kind of knowledge within the company. In addition, IT can also facilitate coordination of NSD activities, which requires IT systems capable of enhancing communication among LSPs employees as well as external actors (e.g. customers and suppliers).

The relevance of information sharing and transparency connected to NSD and the developed service offering is also highlighted in the case study. PN Logistics views distribution and provision of information as an important part of the service offering and considers that this process can be improved both internally and also in the interface to the customers. Transparency of information is perceived as a value-adding component to increase customer value. As illustrated in the above discussion, IT provides a base for LSPs to gather and process information both internally and externally. However, in order to make valuable use of this information, LSPs need to consider the following pre-requisite regarding:

\section{- Provide internal and external information transparency}

To conclude, the relevance of IT has become to conduct an essential part of LSPs' NSD efforts (Zhou \& Wang, 2012), largely due to the fact that many new service initiatives are based on technological developments and innovations (Oke, 2008). From one point of view, IT may receive the role as a critical resource due to its increasing importance in the design and production phase of LSPs' service offerings but also due to its importance as a valueadding component. However, from another point of view, IT can have a more coordinating and integrating role by acting as an enabler to make creative use of information as an important input in the NSD process but also to ensure flexibility and efficiency of relationships and networks in the supply chain. In addition, IT makes it possible for LSPs to create transparency both regarding their NSD network and also connected to their service offerings.

\subsubsection{Internal and external knowledge and skills regarding NSD}

Both within the NSD and the LSP literature, the processes for searching for, identifying and responding to market needs are seen as an important input to a company's NSD (e.g. Alrubaiee, 2013; Menor \& Roth, 2008; Wallenburg, 2009; Ellinger et al., 2008). Despite its relevance, the existence of monitoring the market environment seems to be low among LSPs 
(Flint et al., 2008) and the procedures for it are informal rather than formal (Flint et al., 2005). In addition, prior research shows that customers' expectations of logistics services are higher than the actual receiving (Rajesh et al., 2011). This stresses the need for LSPs to consider how their market orientation efforts can be improved in order to match customer expectations. As mentioned in both the LSP literature and the PN Logistics case study, customers are seen as an important input to LSPs' NSD. However, as Edvardsson et al. (2013) stress, what kind of information and how this is collected and integrated within the company are important factors to consider for NSD.

As highlighted in the PN Logistics case, the different customer needs from the market analysis are matched and prioritised according to the companies' strategic service initiatives and are used to identify themes for reaching a particular customer segment or industry. Processing and being selective towards the sometimes ambiguous customer information and matching these with the LSP's own strategic service initiatives can ensure LSPs develop service offerings in line with their own service strategy. In addition, identifying themes among customer needs allows LSPs to offer more customised service offerings for a specific segment or industry.

In order to identify and make effective use of common themes and trends, LSPs are advised to create systems for collection, analysis, integration and dissemination of the identified customer needs (e.g Wagner \& Franklin, 2008; Flint et al., 2005). In addition, LSPs should also strive to combine different logistics and supply chain expertise with market and sales expertise when conducting and analysing the information from the market orientation (Flint $e t$ al., 2008).

As mentioned before, PN Logistics works in teams in order to utilise the information from the market analysis as effectively as possible. A variety of competences are present within these teams, ranging from skills regarding service development, marketing and communications at a Nordic level. For example, as mentioned in the example with reverse logistics, selected customers and other involved partners and actors were invited to participate and discuss.

As discussed in the NSD literature, service companies can benefit from using different approaches and strategies to achieve an effective market orientation (e.g. Ordanini \& Maglio, 2009; Veflen Olsen \& Sallis, 2006). However, achieving NSD success requires a broader and more proactive approach to market orientation. In general, there seems to be a strong focus amongst LSPs on a more responsive approach to gaining customer insights while less focus was directed at competitor screening, technology developments or different trends or scenarios on the market (e.g. Flint et al., 2008; Wagner, 2013). This may partly be explained as customers being seen as an important source of NSD input, which in turn results in a more reactive approach of LSPs' NSD efforts. However, in order to develop more proactive service offerings, LSPs cannot solely base their NSD input on customers' demands. Thus, LSPs need to identify additional sources for NSD inputs.

PN Logistics actively monitors and measures its competitors' development. This information reveals, for example, PN Logistics' position and development in relation to these actors as well as different service areas that customers perceive as important. Thus, showing awareness and making efforts to screen and follow different technology trends as well as market changes in the supply chain can predict customers' further demands (Gammelgaard, 2008) as well as lead to new service opportunities for LSPs. 
In addition, as revealed in the PN Logistics case, the scope of new service initiatives can range from a bottom-up perspective regarding specific customer needs identified by the sales organisation to a more top-down approach focusing on how PN Logistics can improve its market position within certain areas. According to Shen et al. (2009), LSPs must take their own initiatives in order to continually restructure modes of value creation. This is also in line with the result of Busse and Wagner (2008a), who promote a need for approaches that emphasise and allow openness and proactivity when searching for new ideas.

The discussion above indicates that, in order for LSPs to make effective use of their market orientation efforts they need to develop structured procedures to support gathering, analysing and dissemination of this field-based knowledge within the company. These procedures should also include different responsive and proactive approaches in order to identify different needs on the market. A responsive approach can be seen from a more short-term perspective responding to current customer needs leading to more incremental service adaption and improvements of operational effectiveness, while a proactive approach has a more long-term perspective and provides opportunities to reveal latent customers needs and new market opportunities. The reasoning above leads to the pre-requisite:

- Create responsive as well as proactive approaches for generating, selecting and analysing valuable NSD input

In addition, as mentioned in both the NSD and the LSP literature, in order to make the best use of the collected information, there is a need to evaluate employees' knowledge and skills regarding this. Employees have been identified as having an important role among LSPs and need to be characterised as being multi-skilled generalists rather than technically-oriented in order to develop and provide more value-added services (Jumandi \& Zailani, 2011). As mentioned in the NSD literature, customer orientation requires sales people to understand a customer's entire value chain (Jaw et al., 2010). Furthermore, investments and efforts should be made in order to better understand the customers' multi-faceted role of buyers, payers and users (Michel et al., 2008). By moving towards a more customer-oriented business, this knowledge may be of specific relevance for LSPs. Hence, as a consequence, it will place requirements on the employees to have the "customer in mind" when developing and improving services and systems in order to meet the changing customer needs. In order to provide more complex service solutions, there will be a need for LSPs to gain knowledge about their customers but also to gain information and understand their customer's entire supply chain (Flint et al., 2008).

For example, as mentioned in the case study, to support this kind of knowledge PN Logistics offers its sales people different forms of education as well as tools and systems in order to ensure a quick decision-making process. This is done in order to make the sales people think more holistically and become better at capturing the needs of customers and finding solutions to these specific needs. Thus, in order for LSPs to improve their market orientation knowledge and succeed with this, LSPs will need to train employees for this kind of thinking and invest in efforts in order to understand their customers and their role in the supply chain. This leads to the pre-requisite in order to:

\section{- Support and educate employees for "customer-focused" thinking}

Furthermore, as mentioned in the literature, IT constitutes an important part of LSPs' NSD and is viewed in many cases as the core of the developed service offering (e.g. Third-Party 
Logistics Study, 2013; Pekkarinen \& Ulkuniemi, 2008; Chapman et al., 2003). Due to the importance of IT, it may be relevant to argue that this requires high IT capabilities among LSPs. However, since IT is not regarded as the core of LSPs' expertise, sufficient IT capabilities may not exist within the company. Instead, there may be a need to derive these kinds of capabilities externally through, for example, partnerships with technology companies, which can increase the risk of dependence and loss of relevant knowledge (Oke, 2008). This can also limit LSPs' IT opportunities regarding their NSD efforts. Therefore, LSPs need to strive to maintain an adequately high level of IT knowledge and expertise within the company. For example, increasing employees' understanding regarding the potential IT may offer for NSD is mentioned within the NSD literature as having a positive effect on the effectiveness and adoption of new IT tools (Froehle et al., 2000).

The relevance of understanding the IT possibilities as well as limits can also be illustrated in the case study. PN Logistics states that almost regardless of what type of service development it is, it will require some type of IT dependency and usually require some type of change in the production as well. As a consequence of this, PN Logistics emphasises the importance of understanding and considering the impact on the IT support if a change is made in the production.

Based on the above discussion, in order for LSPs to fully benefit from their IT investments as a way to improve its NSD efforts, there may be a need to increase understanding among employees regarding its NSD potential as well as its influence and impact on the NSD process. Thus the next pre-requisite regarding NSD knowledge and skills is as follows:

\section{- Increase internal understanding of IT's NSD possibilities and limits}

Furthermore, in order to gain more knowledge for NSD inputs, LSPs are recommended to engage in more NSD collaboration efforts with external actors (Wagner, 2013). But the question is how to find these actors? As mentioned in the NSD literature, customer orientation should be advanced more quickly in order to understand customer information and respond faster to customers' needs by providing new service offerings (Jaw et al., 2010). This in turn can place requirements on LSPs to segment both existing customers and also non-customers in order to develop appropriate service offerings for different profiles of customers, but also to strengthen relationships or loosen the constraints from becoming customers. As mentioned in the case study, PostNord has an overall strategy to continue to grow with existing customers. This applies to all the different business areas and PN Logistics states that:

\section{"The most important thing is to grow with our customers."}

In order to realise this goal, PN Logistics has selected a number of customers who have operations in many countries, since this is an area where PN Logistics believes it has potential to grow. Since PN Logistics can offer more complete solutions than before, this also leads to new business opportunities with customers who were previously interested in these kinds of solutions. These new customers are reached either by using existing portfolio of potential customers or by using existing databases where customers have been divided into different sectors/industries and segments in order to find "twins" to existing customers. Due to the size of the organisation and involvement of hundreds of sales people, this work has to be done in a systematic and structured way, as well as developed IT support concerning CRM. In addition, in order to take advantage of each other's knowledge and skills throughout the organisation, PN Logistics aims to develop a uniform way of how this should be done and form a strategy 
that will be applied to all operational units. This is also supported by Wagner (2013), who highlights the need for LSPs to create and develop internal processes and capabilities in order to identify external NSD sources as well as collaboration partners.

However, as mentioned in the LSP literature, there seems to be a low utilisation of external partners among LSPs' NSD efforts and few LSPs engage in logistics innovation with their customers (e.g. Wagner, 2013; Flint et al., 2008). However, it is suggested that integration with customers leads to several positive outcomes of the NSD efforts as well as strengthening the position of LSPs and their relationships with customers. This can be supported in the case study. PN Logistics tries to integrate the customers during the development process, especially when it comes to strategic initiatives. In addition, customer participation is seen as a necessity when things need to be tested at different stages in the NSD process since it is hard to predict all possible outcomes in advance. Hence, LSPs might consider additional ways of how to integrate more with their customers, as well as how, and at what point, they should be involved in the NSD process (Flint et al., 2008). In addition, LSPs also need to consider for what reasons and also for which strategic service initiatives, in order to fully benefit from these NSD collaborations efforts. The reasoning above leads to the following pre-requisite:

\section{- Identifying and integrating customers for reaching strategic NSD initiatives}

Even though customers are considered as an important source of NSD input for LSPs, there may also be a need for LSPs to consider NSD input from other external actors as well as to identify new collaboration arrangements for their NSD efforts. For example, Wagner (2013) reveals that in addition to customers, suppliers and competitors are seen as a source to service improvements; however, these actors were not directly related to services new to the firm. Collaboration with consultancies or universities was found not to have a positive impact on service improvements nor on development of new services. However, despite the low utilisation of external actors, the author stresses the relevance of both direct and indirect input from these actors for LSPs' NSD efforts.

Due to the evolving development of logistics service offerings both in geographical scope and complexity, integration of external actors can be of particular relevance for LSPs' NSD efforts in the future. As mentioned earlier, in the PN Logistics case, one example of using different actors' input for NSD efforts can be illustrated with the work connected to reverse logistics. Reverse logistics is an area that has recently been developed and changed, much depending on everything that happens within the e-commerce business. PN Logistics perceives that new kinds of customer needs have started to emerge, and since transports have become more international, the question of returns is becoming more complex, in particular if customs are involved. To be able to respond to these needs, interactions with involved partners and other actors such as developers of TA (Transport Administration) systems, as well as customers, has been initiated. These efforts have resulted in a number of possible service initiatives within the reverse logistics area.

In addition, the ability to use and integrate external partners in the NSD process is mentioned in the LSP literature as a competitive advantage that distinguishes one LSP from another (Wagner, 2013). Due to the fact that LSPs' service offerings tend to become more complex and knowledge-intensive, the necessary knowledge and skills for these service offerings may not exist within their own company. This places requirements on LSPs to identify potential collaboration partners that can match these knowledge areas, as well as how these can be integrated in the NSD process. Thus, LSPs need to be aware of the impact such collaboration 
efforts may have on their NSD outcome and therefore need to consider the pre-requisite regarding:

- Identify the need for and benefits of external interfaces or collaboration partners for meeting new customer demands

To conclude, a service company's ability to identify and respond to market needs is described in the literature as playing a crucial role and can be seen as the core or point of departure for the NSD efforts (e.g. Menor \& Roth, 2007; Sundbo, 1997). Due to the criticism of the LSP industry for being more reactive than proactive in its NSD efforts and low monitoring of the market environment, it may be relevant to stress the importance of NSD knowledge and skills among LSPs. As indicated in the analysis above, an LSP's ability to create pre-requisites regarding NSD knowledge does not solely concern identifying different external sources of NSD input or how this information is used and integrated in the NSD process. Instead, it also concerns LSPs' pre-requisites in identifying and supporting existing knowledge and skills regarding NSD within their own company. Thus, the role of NSD knowledge and skills may be described as the kernel that feeds and fosters the development of new service offerings further. This may not be unique for LSPs, but rather its importance may be relevant for other service providers as well. However, as suggested by Wagner (2013), the ability to use and integrate external knowledge and skills in particular into NSD activities can provide a competitive advantage for LSPs and distinguish one actor from another.

\subsection{Analysis II - linking the different NSD dimensions to each other}

The second section in the analysis addresses RQ1c, which aims to investigate the link between the different dimensions with their underlying pre-requisites to each other and describe the relation between them. The logic of this section is to guide the reader step by step in the development of an LSP's NSD framework, which is presented as a whole at the end of this section.

First, the analysis starts with the two NSD dimensions that were identified in the first part of the analysis as the foundation for an LSP's NSD, namely the dimensions NSD culture and NSD strategic focus, see Figure 7.2 below.

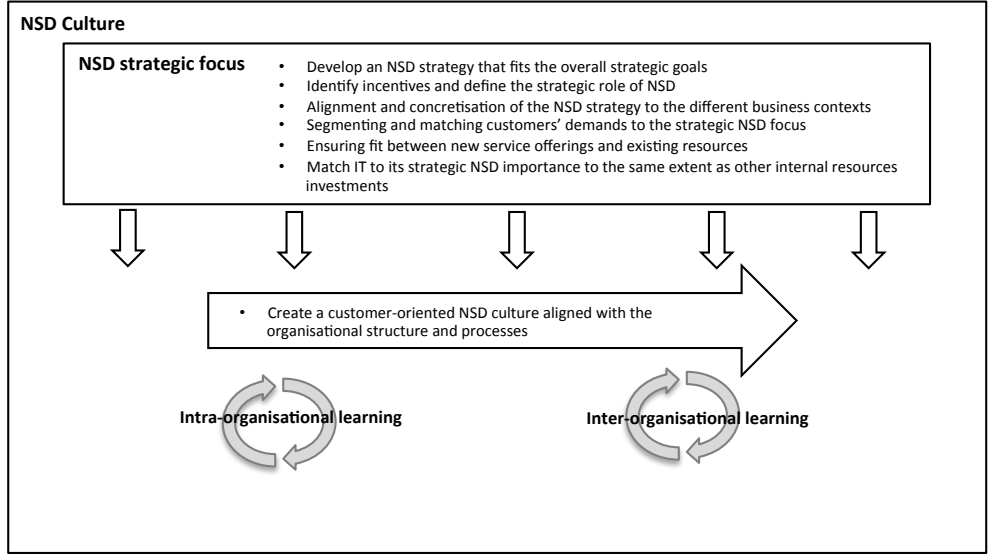

Figure 7.2 The link between NSD culture and NSD strategic focus. 
The LSP industry has earlier maintained a more production-oriented approach in its operations and activities. However, LSPs' increased strategic role in the supply chain has started an expansion of the scope of LSPs' service offerings to provide the entire logistics needs for their customers (Chapman et al., 2003). As a consequence, there is pressure on LSPs to fully understand the entire supply chain of their customers (Pekkarinen \& Ulkuniemi, 2008). Therefore, in order to respond to the market pressure there may be a need for LSPs to become more customer-oriented in their NSD efforts. This orientation is here defined as an NSD Culture aspect.

For example, having different NSD cultures can be illustrated in the case study, where the operational unit in Norway has a more customer-oriented approach and the operational units in Sweden and Denmark have a more production-oriented approach. As mentioned in the case study, these two approaches have different impacts on the organisation, managerial priorities and NSD efforts. However, PostNord strives to become more customer-oriented in their operations and there is a strategic NSD focus on continuing to grow with existing customers as well as offer more end-to-end solutions to its customers. This applies to all the different business areas within PostNord. As a first step towards this goal, a target group involving business areas managers and key account managers from the different business areas has been selected to find potential solutions that go beyond the boundaries of the different business areas. As mentioned in 7.2.1, the trend towards broader and more complex logistics solutions is not specific for PostNord, and it will place requirements on LSPs in general to find synergies and improve internal coordination and collaboration in order to use internal resources and knowledge effectively. This can be supported in the PN Logistics case, in order to become more transnational in their service offerings, efforts are made to help sales people in the different operational units get to know each other and share knowledge. In addition, an increasing customer focus and developing more customised service offerings will also affect an LSP's external collaboration and coordination with its customers. For example, PN Logistics sees customer participation as a necessity and tries to integrate customers during the service development process, especially when it comes to the strategic initiatives.

In section 7.2.2, the strategic focus and strategy of NSD is described to have a central role of LSPs' NSD efforts. This is in line with Zhou and Wang (2012), who argue that the NSD strategy can be considered as the centre position among all the different activities related to NSD. Based on the above discussion, it can be suggested that an LSP's NSD strategic focus seems to affect the NSD culture of how to manage and coordinate NSD activities and resources within the company. On the other hand, the NSD culture of, for example, promoting intra- and inter-organisational learning within the company can be seen as a foundation to support and fulfil the strategic NSD focus. It can thus be argued that the role and connection between NSD culture and NSD strategic focus constitute the foundation and centre of an LSP's NSD efforts and thus have an impact on the other NSD dimensions mentioned in this study.

The next step in the framework concerns LSPs' process focus on their NSD efforts. The identified pre-requisites regarding this dimension are illustrated in Figure 7.3 below. 


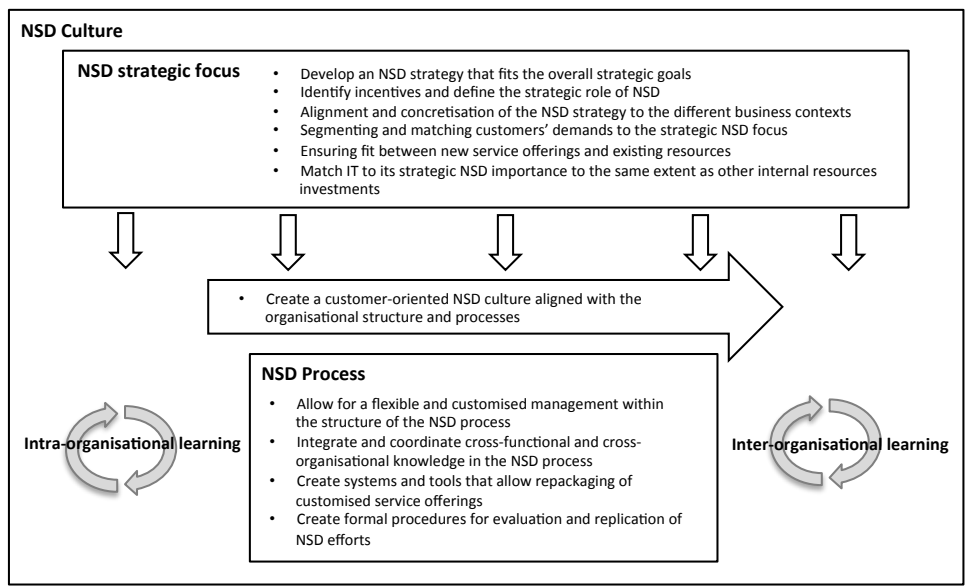

Figure 7.3 NSD strategy, NSD culture and NSD process.

As mentioned in 7.2.3, the existence and formalisation of NSD processes and structures can be described as a fundamental structure, in order to enable and facilitate the management, evaluation, learning and improvement of its included steps and activities. This can be illustrated in the PN Logistics case regarding, among other things, efforts to identify new customers. Due to the fact that PN Logistics can offer more complete solutions than before, this leads to new business opportunities with customers who were previously interested in these kinds of solutions. Due to the size of the organisation and involvement of hundreds of sales people, this work has to be done in a systematic and structured way. PN Logistics aims to develop a uniform way of how this work should be conducted and form a strategy that will be applied to all the countries in the Nordic region. This is done in order to take advantage of each other's knowledge and competences throughout the organisation.

The example above indicates that an LSP's strategic NSD focus as, for example, mentioned by PN Logistics to provide more end-to-end solutions, will put demands for NSD processes and structures needed in order to support the strategic NSD focus and its including activities. It also reveals the relevance of standardised NSD structures and processes in order to use their resources and knowledge as efficiently as possible and promote intra-organisational learning. In addition, the need to adapt processes and structures may also be relevant when integrating external resources and knowledge into the NSD process. PN Logistics tries to integrate the customers during the service development process, especially when it comes to strategic initiatives. Customer participation is seen as a necessity when things need to be tested at different stages in the process since it is hard to predict all possible outcomes. However, in order to work more flexibly and allow a larger degree of testing, the existing project methodology is to be replaced within PN Logistics. As with internal resources and knowledge, an LSP's NSD process should also support integration of external resources and knowledge, which in turn can promote an inter-organisational learning approach and thereby influence the NSD culture.

Moving further in the framework to LSPs' NSD knowledge and skills. As illustrated in Figure 7.4 below, this dimension has been divided into internal knowledge and resources and external knowledge and resources. 


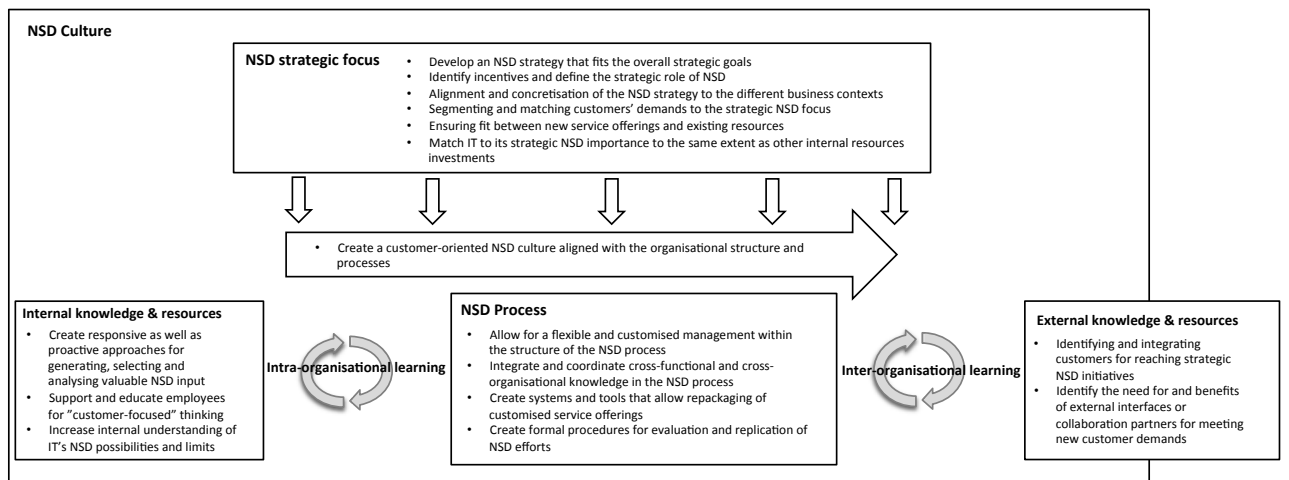

Figure 7.4 NSD culture, NSD strategy, NSD process and NSD knowledge and skills.

In order to meet market demands and discover new business opportunities, Chapman et al. (2003) stress the need for LSPs to search frequently for new knowledge. Hence, this places requirements on LSPs regarding how to gain valuable information from different sources and how this information is processed and analysed, as well as spread, within the company. As mentioned in the analysis above, an LSP's focus on NSD processes and structures can have an essential influence on the use of both internal and external NSD knowledge and resources.

The role of gaining external NSD knowledge can also have an influence on an LSP's strategic NSD focus. For example, as mentioned in the case study, several of the major strategic initiatives within PN Logistics that have been established are based on a dialogue with the customers and their needs. There is on-going work with major customers where meetings with different focus areas, such as operational, tactical and strategic issues, are held on a regular basis. These meetings often result in various issues that must be addressed and further developed. Thus, an LSP's NSD knowledge and skills may have an influence when setting the strategic focus of NSD. However, the NSD strategic focus may also affect the content of NSD knowledge and skills by identifying both knowledge and resources for reaching the strategic NSD incentives. This applies to knowledge and resources both from an internal and an external perspective.

In addition, as mentioned in 7.2.5, an LSP's NSD knowledge and skills, both internal and external, can be described as the starting point as well as a force that improves and drives NSD activities and the development of service offerings further. However, in order to manage and make effective use of this NSD knowledge and skills, there is a need for a certain focus on NSD processes and structures as mentioned in the above discussion.

The final NSD dimension in the model concerns IT use and expertise, see Figure 7.5. This dimension has been divided into synchronisation of IT systems and information transparency, which were identified in section 7.2.4 as two pre-requisites on which it is essential for LSPs to focus for their further NSD efforts. As mentioned in the LSP literature, the evolving IT trend can be seen as a driver for growth as well as a source and driver for NSD opportunities for LSPs. Due to additional trends such as increased globalisation, digitisation as well as internationalisation affecting the logistics market, it may be relevant to argue that the role of information has an increasing importance for both LSPs' business and their NSD efforts. 


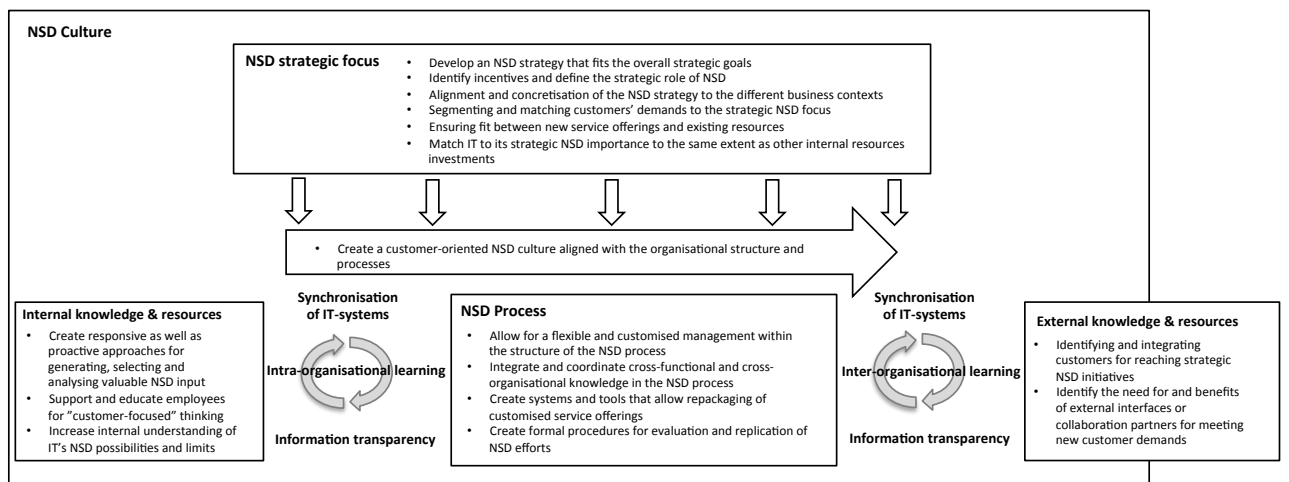

Figure 7.5 A framework for LSPs' NSD.

Moving towards more customised services offerings will increase the pressure on LSPs' NSD pre-requisites regarding gaining information from external sources as well as interaction with external partners in the supply chain. A precondition for making effective use of this information and interactions is thus integration and synchronisation of IT systems. This is needed in order to support LSPs' daily activities but also in order to create opportunities by providing added value to customers through different IT solutions. The link between synchronisation of IT systems and its influence on the NSD process is also identified in the PN Logistics case. The ability to be more effective in the process from an idea to action is something that PN Logistics is striving to be better at managing. However, due to a very fragmented portfolio of IT systems, together with the restriction of reducing IT costs, this is not perceived to have a positive effect on increasing the speed of the service development process. However, PN Logistics mentions that it is often a matter of making priorities and that IT resources are available but not unlimited. This is turn points to the relevance for LSPs to map and define IT resources needed to support their NSD efforts. In addition, due to the potential and benefits IT can generate for LSPs' NSD efforts, the connection and match between the IT resource investments and LSPs' strategic NSD focus may be considered.

In addition, it can also be argued that the synchronisation of IT systems influences NSD knowledge and skills by facilitating information sharing within the organisation. As mentioned in the PN Logistics case, the idea behind the Track and Trace solution was that it should provide the same type of information in all the different countries. The synchronisation of IT systems can also be relevant for LSPs from an external NSD knowledge and skills perspective. Increased information exchange between LSPs and their customers through IT systems can provide opportunities for LSPs to increase the knowledge of their customers' supply chains. In addition, PN Logistics highlight that building more electronic connection with customers can lead to stronger relationships.

However, this in turn will place requirements on LSP pre-requisites regarding processing this kind of information, as well as making it transparent. The importance of information transparency can affect LSPs' NSD on many different levels. Due to the fact that the LSP industry is characterised by already low margins and a high focus on cost reductions, information can be a way to add value to their customers. This is something that is supported in the case study, where PN Logistics highlighted the important role of the provision and distribution of information as an essential part in the service offering. 
In line with customers' increasing demands for information exchange regarding service delivery as well as costs, there are thus increasing requirements on the monitoring and traceability of LSPs' customers' logistical flows. However, this in turn places requirements on LSPs' NSD knowledge and skills regarding the analysis and effective use of this information, since it can be a way to provide customised service offerings to customers.

As mentioned in section 7.2.4, an LSP's IT use and expertise can be seen as having a coordinating and integrating role by acting as an enabler for gaining and disseminating both internal and external NSD knowledge and skills. In addition, as mentioned in the above discussion, it is also regarded as an important component in the service offerings itself, as well as in the process of designing it.

To sum up, the developed framework, see Figure 7.5, aims to reveal a holistic view of LSPs' NSD. The different NSD dimensions, together with the underlying pre-requisites derived from the literature together with the case study, strive to illustrate areas that are essential for LSPs to consider and to focus on when developing new service offerings. An overlying trend in the developed framework is the focus on internal IT development and increased customer knowledge and integration. All the different NSD dimensions have their own purpose and provide a partial understanding of LSPs' NSD. However, as found in section 7.3, the different NSD dimensions have a certain connection to and influence on each other, which suggests that in order to understand NSD there is a need for LSPs to take a more holistic view of their NSD efforts. The identified links between the different NSD dimensions can rather be described as reciprocal than sequential, which in turn even further stresses the complexity of NSD and its management. In line with Li et al. (2005), NSD can be described as a network, where each stage is dependent on the others and is driven by the feedbacks between the different stages.

\subsection{Analysis III - NSD framework from a green perspective}

The third and final section of the analysis addresses RQ2 in this dissertation. The analysis of this section takes its starting point in the developed NSD framework presented at the end of section 7.3, see Figure 7.6 below. This section is structured according to the identified NSD dimensions when analysing the implications in the development of green service offerings among LSPs. The analysis in this section is mostly empirical driven, based on input from the case study focusing on the environmental work at PostNord together with the results from the Licentiate thesis and its multiple case study. In addition, literature findings and empirical findings in Chapter 5 have also been used as an input to this analysis. 


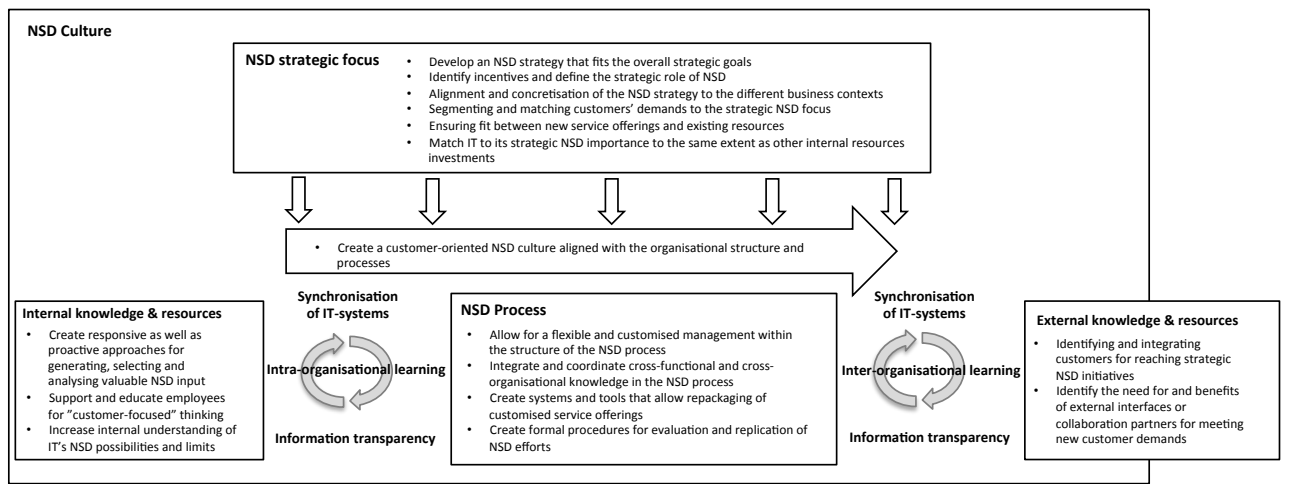

Figure 7.6 The developed NSD framework.

\subsubsection{NSD Culture}

As identified in section 7.2.1, when LSPs start to develop green service offerings it may also be relevant that they strive to create a customer-oriented NSD culture in order to encourage employees to think of the customers as well as promote intra- and inter-organisational learning. However, in similar reasoning regarding becoming more customer-focused in its NSD activities, there may also be a need for a culture or climate that promotes more "green thinking" when developing green service offerings. Creating a culture that encourages employees' green behaviour, making knowledge and resources available as well as promoting organisational learning, is mentioned in the literature as having a positive influence on LSPs' general adoption of green initiatives (e.g. Lin \& Ho, 2011; Zailani et al., 2011).

One way to encouraging employees' green behaviour can be found in the case study regarding PostNord's initiative with the Climate Fund, where the employees from the different business areas can submit proposals in order to improve environmental and climate measures in their own operations. One of the aims of the Climate Fund is to create participation and commitment regarding environmental issues within the organisation. This initiative does not directly concern development of green service offerings; however, motivating and encouraging employees to start thinking green in their daily operations can be regarded as a cultural aspect towards the development of how their environmental work can be integrated in their service offerings.

Similar reasoning can be found in the study by Zailani et al. (2011), who argue that the creation of a climate that allows and supports green knowledge sharing within the organisation can lead to greater realisation of an LSP's green innovations efforts. In a way, it can be said to concern creation of a "common picture and language" among employees regarding their environmental work as well as the implications for the service offerings. A similar result can be found in Paper 2 in the Licentiate thesis, where lack of understanding and interest in the organisation, different processes in different parts of the company and lack of environmental competence were identified as barriers to LSPs' environmental work. However, this issue concerned first and foremost the large LSPs studied, with different units and global units within the organisation. Hence, the result from Paper 2 indicates that organisational resilience is correlated with information dissemination and shows thus how important it is for LSPs to be clear about the incentives for their environmental work within the organisation. In addition, the creation of a "common picture" within the organisation may be perceived as equally important when it comes to developing green service offerings. 
This can be supported by PostNord's work to consolidate the different business areas and commonly discuss service development from a green perspective. This discussion does not include precisely how the service or the product will look; rather, it is more about which characteristics green service offerings should consist of and what the framework should be. The aim of this work is to develop common environmental criteria which the different business areas can relate to within their own service development efforts. In this way, benefits are reached by using each other's knowledge of green expertise and service development skills.

However, creation of a culture or climate that encourages employees to think "green" as well as promoting learning of existing green knowledge within the company, are suggested in the literature to have a correlation with organisational and top management support (e.g. Zailani et al., 2011; Lin \& Ho, 2011). In addition, Abareshi and Molla (2013) argue that an LSP's exploration of green knowledge reflects the support of top management for these kinds of practices. Similar results were also found in the Licentiate thesis, where engagement and support from top management can be crucial for how successfully the adoption of green initiatives are integrated in the company as well as received by the employees. In addition, as found in Isaksson et al. (2012), strategic presence and top management support seem also to be related to cross-functional green involvement within the LSPs studied. Thus, there seems to exist a connection between the strategic environmental focus and how transfer and sharing of green knowledge is organised within the company.

To conclude, creating a culture that promotes participation and commitment regarding green initiatives is highlighted both in the literature and in the empirical data. Such a culture is also suggested to benefit and promote the development of green service offerings. In addition, creation of a "common picture" can be viewed as equally important in the development of green service offerings, in order to unite different business areas towards a common goal and also to take advantage of each other's green knowledge and service development skills. In addition, compared to LSPs' NSD culture in general, the role and support from top management is highlighted in the analysis to have a significant impact regarding the creation of a green culture. As suggested in the analysis above, top management's support can influence employees' green awareness and attitude, sharing and learning of green knowledge as well as the existence of cross-functional involvement regarding green initiatives.

\subsubsection{NSD Strategy}

Earlier research indicates that LSPs have an ambition to improve their green efforts (Zailani $e t$ $a l ., 2012$ ) and that there is a need for LSPs to incorporate environmental considerations into their overall business strategy in order to strive for a higher level of green performance (Abareshi \& Molla, 2013). This can be illustrated by the results in the study by Isaksson et al . (2012), which indicate that existence of green concerns (both formally and informally) in the LSPs' overall business strategy has a positive correlation with the implementation of green initiatives. Moreover, as suggested in the results of the Licentiate thesis, the strategic priority regarding green issues can serve as an indicator of a company's level of adoption of green initiatives. But the question to ask is how this influences the development of green service offerings. As found in the literature review in Paper 1 in the Licentiate thesis, integrating sustainable product and service development as one element of the existing corporate strategy will require new kinds of thinking in existing structures and systems.

An example of integrating green aspects into the service development and the match to the company's overall strategic goals can be illustrated in the PostNord case study. In order to 
further integrate green aspects into the business, PN Logistics has recently started to work with establishing a new brand for the business unit, where environmental considerations will be one of the core values. PN Logistics argues that much environmental work has already been done but has not been reflected in the daily work, on the service side. However, PN Logistics argues that the core values of PostNord as an available and reliable business partner as well as being environmentally friendly in its operations should to a large degree characterise the service offerings as well as the development of these.

Regarding the status of developing green service offerings among LSPs in Sweden, the result from the Licentiate thesis revealed that the LSPs studied were characterised as either offering green choices connected to general service offerings, or a full integration of green thinking in the company and its service offering. As mentioned in the case study, the decision was previously taken within PostNord not to offer its customers specific green service offerings even though there have been some green added services within the different business units. The strategy has been to improve the environmental performance throughout the organisation and the company has hoped that this would satisfy customers. However, the company perceives that its customers have started demanding green service offerings, which leads to considerations regarding how PostNord can meet these requirements.

This in turn can reveal the relevance for LSPs of having a clear incentive for their environmental work as well as integrating it in the service development and in the offering itself. As mentioned in the case study, there may be different incentives for environmental work in general, than the incentives for the development of green service offerings. The incentive for PostNord's internal environmental work is to reduce its environmental impact while the incentives for developing green services are to meet customer needs and also to tie the customers closer to the company. The general environmental work is an approach that PostNord cannot let go of due to the risk of not achieving the set environmental targets, but the company must also begin to meet customer requirements for green service offerings. As the Environmental Manager of PostNord states, they have to find a balance to do both.

Thus, depending on the LSPs' incentives for developing green service offerings, it may be relevant to argue that this may have a certain influence on the continued development of these kinds of service offerings. As mentioned by PN Logistics, the work to integrate environmental values in the service development is to a large extent based on requests and requirements from customers. Previously, PN Logistics has chosen not to offer its customers specific green service offerings; however, the possibility of providing green service offerings in the future is under consideration. The vice president of Business Development, PN Logistics states:

"...the main focus cannot just be about carbon offset; instead it must contain something of real value for the customer. You have to make real actions out of your promises and also make demands on the customers as well. On the other hand, being more effective from a green perspective usually goes hand in hand with reduced costs as well. For example, by increasing the fill rates, positive outcomes will be reached both from a green and a cost perspective."

Another aspect found in the PostNord case concerns identified differences regarding the environmental work in general within the company, which in turn will probably also have implications for the development of green service offerings. Similar findings can be found in the Licentiate thesis, which reveals that an LSP's specific firm characteristic in combination 
with its business context may have an impact on the adoption of green initiatives within the company. Thus, due to a generally broad scope of service offerings together with a customer base representing various industries, especially for medium and large sized LSPs, this may also be an internal matter. As found in the PostNord case study, the environmental work, as well as providing different types of green service offerings, varies between the different business areas within PostNord. For example, Mail Sweden has a different approach as well as other conditions and customer opportunities regarding their environmental work compared to, for example, PN Logistics.

This reveals the need for LSPs to adjust their environmental work and development of green services for specific business conditions as well as customers' needs and requirements. Regarding customers' environmental requirements, both the PostNord case study and the multiple case study in the Licentiate thesis indicate that customers from different industries place varying pressures and requirements on LSPs regarding green service offerings. For example, within Mail Sweden, the customers who are public organisations have very tough environmental requirements. Having different approaches regarding addressing green offerings to customers was also identified in Paper 3 in the Licentiate thesis. The LSPs studied either chose to address their green offerings to all or a wide range of their customers, or addressed focused customer segments with their green offerings.

In addition, segmenting customers based on the environmental aspect (environmental awareness, ambitions and willingness to pay) was mentioned in Paper 1 (in the Licentiate thesis) as an important factor when developing green service offerings. Within PostNord, there is no structured work regarding customer segmentation with respect to the environment. However, the environmental manager of PostNord has tested doing this for the largest customers. The selected customers were placed on a scale with environmental performance on one axis and environmental image on the other axis. By doing this, it was possible to group those customers who have a good environmental performance and a genuine environmental effort and those customers who have a strong environmental image. Segmentation of customers regarding their environmental efforts and image can thus lead to valuable NSD input by increasing the knowledge and understanding of customers' green attitudes and requirements.

Furthermore, an LSP's general environmental work can also be seen as a platform which can both facilitate and/or hinder an LSP's development of green service offerings. This can, for example, be revealed in the PostNord case study. Achieving the environmental goals set will require a variety of initiatives and actions, such as running on more energy-efficient modes of transport and building new terminals in order to increase mail volumes on trains. Another initiative mentioned is connected to the opportunity to use alternative fuels. Within Mail Sweden, it is easier to find alternative vehicles due to the greater use of light vehicles. However, when it comes to heavier vehicles, it is more difficult to find alternative fuels or alternative vehicles at all. From this point of view, depending on an LSP's range of business, this can create different conditions for developing green service offerings.

Furthermore, as revealed in the case study, there are a variety of underlying activities within PostNord to improve environmental performance. However, this is something that is not always mentioned or shown to the customers. Many of the environmental requirements that customers want to discuss come down to a fairly operational level and involve specifications regarding types of tiers, alternative fuels for transports as well as environmentally classified vehicles. These are activities that PostNord perceives itself as doing well, largely because it 
has its own vehicle fleet and can therefore control and ensure that quality and environmental issues are taken into account. However, as the Environmental Manager of PostNord states regarding possibilities of green service offerings within the logistics area:

\section{“...much of the work is about packaging and communicationg what we already do...”}

To sum up, the analysis above reveals the strategic importance of inclusion of environmental aspects in the business strategy, since it is positively related to adoption of green initiatives within the LSPs studied. In addition, the existence of green service offerings seems also to be strategically related. However, the strategic incentives between the existence of green service offerings and environmental work in general may differ which in turn calls for a need for LSPs to specify their strategic ambitions towards development of green service offerings. In addition, as revealed in the analysis, customers seem to have a significant impact on this service development. This in turn calls for an increased effort to identify, gain and analyse data about customers' green demands. The discussion above also indicates that an LSP's general environmental work can be seen as a resource base for its further development of green service offerings. However, the analysis revealed that different LSPs as well as different business units within large LSPs can have different opportunities in form of available resources and customers' requirements. Thus, LSPs will need to match existing resources and knowledge regarding their general environmental work towards their customers' green requirements in order to convert environmental efforts into green service offerings.

\subsubsection{NSD Process}

Regarding the process focus, LSPs are advised to develop different kinds of routines, practices and processes in order to integrate and transform different kinds of environmental knowledge and thus improve their green performance (Abareshi \& Molla, 2013). In addition, the authors reveal that those LSPs that adopt effective routines and processes would gain better capabilities to integrate green knowledge into their logistics operations. It may also be reasonable to argue that adoption of effective routines and processes regarding the environmental work has a positive influence on the development of green service offerings.

Thus, developed routines and processes can be a way to increase both the transparency and the credibility of service offerings to customers. In addition, as mentioned in Paper 1 in the Licentiate thesis, adoption of certain certifications and standards can be a way to visualise an LSP's environmental work and thus strengthen the credibility of their service offerings. For example, environmental certifications (such as ISO 14001) received the highest mean in the questionnaire survey (see Table 8 in Chapter 5). Similar results can be found in the Nordic survey study conducted by PostNord (2012). The results reveal that ISO certification is the most frequent requirement (46\%) among customers and has increased by $18 \%$ since a similar survey study in 2010 .

In addition, another aspect that reveals the relevance of environmental standards concerns environmental calculations and documentation. This aspect was found in both the PostNord case study and the multiple case study in the Licentiate thesis. For example, PostNord has realised that the environmental aspects are becoming increasingly important and has noticed increased requirements for environmental reporting. In addition, offering documented emissions data was also one of the most commonly adopted measures among LSPs in the questionnaire survey, see Table 7 in section 5.1. However, even if calculating and documenting environmental data seems to be common among LSPs, there does not seem to 
be a standard way to do this. This problem was also mentioned in the PostNord case study. The fact that different logistics operators calculate the environmental numbers differently can make it difficult for customers to compare these figures between different actors. The Environmental Manager of PostNord states:

"We need a standard way to calculate the environmental impact of transports in order to make it possible for customers to compare their choices and it is only then a lower environmental impact can determine the choice between two actors."

As mentioned in the result from the Licentiate thesis, the lack of standardised methods and vague robustness of these calculations may have a negative influence on LSPs. Customers may perceive that these figures are not accurate enough and therefore be unwilling to pay extra for these kinds of service offerings. However, PostNord tries to counteract this scenario by being engaged in various industry forums in order to provide uniform environmental calculations which create an opportunity for customers to compare different LSPs and see which is the most environmentally efficient. Similar results regarding a lack of a standard methodology concerning measuring environmental impact can also be found in the study by Colicchia et al. (2013). Thus, the authors stress the need for further research to focus on the creation of effective performance measurement systems for allocating different environmental impact to different customers and suppliers. However, measuring environmental performance has been shown not to be an easy task. As mentioned in the PostNord case study, more detailed follow-up procedures would be necessary to achieve more accuracy, as well as technical investments such as vehicle computers in all cars. In addition, the boundaries of the considered systems may also have an impact of the outcome of these environmental figures.

Furthermore, as Abareshi and Molla (2013) mention, adoption of processes and routines such as environmental training programmes and implementation of life cycle analyses can act as facilitators for LSPs' success in transforming different kinds of knowledge and information into green routines and practices within the company. Developing processes and procedures for the environmental work may benefit LSPs from both an internal perspective as well as from an external perspective.

For example, as mentioned earlier in this analysis, within PostNord there is no structured way to segment customers' environmental work, but doing this can give an indication of customers environmental requirements. In addition, by adopting a similar approach it can also be possible to adapt the environmental communication for a specific customer target group and try to package different types of service offerings to these. It can also be seen as a way to help customers that have good environmental performance to also improve their environmental image. The Environmental Manager of PostNord believes doing this may result in an active dialogue and sales effort to tie the customers closer and also strengthen the whole affair. The Environmental Manager of PostNord states:

\section{"Much of this work, however, is based on what you know about the customers and also based on sure instinct."}

To sum up, the above discussion reveals some potential benefits of a process focus both from an external and an internal perspective. The adoption of different certifications and standards, for example, developing industry standards regarding LSPs' environmental work, can help to increase transparency and strengthen the credibility of LSPs' environmental work as well as their future green service offerings. In addition, the discussion also suggests the relevance of 
developing internal procedures and routines in order to collect, transform and integrate environmental knowledge into LSPs' operations as well as service offerings. Thus, the next step concerns different types of internal and external knowledge sources LSPs can use for their environmental efforts as well as their service offerings.

\subsubsection{NSD Knowledge and Skills}

In the study by Abareshi and Molla (2013), the result reveals that there is a greater focus on internal than external environmental knowledge among LSPs. However, in order to develop green practise, the authors argue that LSPs need to improve their ability to value and use both internal and external environmental knowledge. The following section is divided into different sub-sections regarding LSPs' NSD knowledge and skills from a green perspective.

\section{Internal knowledge \& resources}

Both in the PostNord case study and the multiple case study in the Licentiate thesis, employees are seen as an important driver and resource for the environmental work. As mentioned in both case studies, adopting a green business approach seems partly to rely on a desire both to be viewed as an attractive workplace as well as to increase the ability to attract new employees who are more value-driven. The view of integrating green thinking throughout the company and involving the employees in this process was specifically mentioned by one LSP in the multiple case study in the Licentiate thesis. The LSP studied states that:

"We work a lot to increase personal responsibility and willingness to contribute to the whole company. We think this is a winning concept."

Furthermore, the same LSP states:

"We want to educate our employees so they can support our customers and everyone in the organisation should know where we stand when it comes to sustainability issues."

The results from Paper 3 and Paper 1 in the Licentiate thesis showed the significant role of employees as co-creators of green value as well as highlighting the relevance of offering employees training and education in order to increase the level of green awareness and knowledge. As mentioned in Paper 1, training in green issues, information and the spread of codified and tacit knowledge can facilitate the integration of environmental concerns into service/product development process. Thus, in order to encourage and support the employees' green engagement and attitude, LSPs are advised to offer employees different kinds of environmental training and education (Lin \& Ho, 2008). For example, as found in the PostNord case study, training drivers and postmen in eco-driving is seen as an important part of the company's environmental work. In addition, eco-driving was also found in the questionnaire survey to be one of the most commonly adopted green initiatives among LSPs in their efforts to improve the environmental impact.

Moreover, as can be found in the study by Maack (2012), education of employees within sales and operations is seen as important in order to increase the level of environmental knowledge especially connected to the service offerings. Similar observations can also be illustrated in the PostNord case study. The company sees the environment as a future competitive advantage but it will probably be required to be more clearly integrated in the sales process. The sales staff need to become more confident in talking about environmental issues with customers without bringing in an environmental specialist. However, the situation looks 
rather different between the different business areas. Mail Sweden has come a long way in the work of educating the sales force. A potential explanation, according to the Environmental Manager, is that the business area has existing bundled environmental services within the Climate economic product family. The Environmental Manager of PostNord states that:

\section{"In order to be able to sell these kinds of service offerings, you have to understand the underlying reasons behind them."}

As mentioned by Maack (2012), sales people can have a major marketing role towards customers by revealing some of the intangible benefits connected to an environmental initiative or service offering. Or as Gammelgaard and Prockl (2012) choose to point out: employees can transform a company's environmental efforts into reality. However, as indicated in the above discussion, in order to reach that state it is necessary to educate and train the employees in thinking green and understanding the implications, as well as translating environmental efforts into something concrete.

Another example which shows the relevance of creating procedures and approaches for generating and analysing internal environmental knowledge can be illustrated in the PostNord case study regarding their city logistics concept. As mentioned in the case description, a project committee has been selected in order to partly be responsible for coordinating a number of workshops in order to identify and collect all knowledge and information within the organisation within the field of city logistics. This is done in order to utilise benefits from previous experiences within this field but it also acts as an input to further work for defining and shaping the city logistics area. Parallel to this work, there is an on-going dialogue to reconcile the existing city logistics initiatives in each of the different business units, where employees have the chance to make an impact. Thus, the aim with these initiatives does not solely concern identifying and sharing existing knowledge, but also creating an engagement among employees. As mentioned by Abareshi and Molla (2013), those LSPs that adopt effective practices and processes to obtain different kinds of environmental knowledge would gain better capabilities to integrate this kind of information into their operations. Hence it may also be reasonable to argue that this kind of information and knowledge constitutes a basis for ideas about the development of new service offerings from a green perspective.

\section{External knowledge and resources - customers}

Many researchers in the reviewed literature stress the need and relevance for LSPs to identify external sources of knowledge and resources regarding their environmental work (e.g. Colicchia et al., 2013; Rossi et al., 2013; Abareshi \& Molla, 2013; Gammelgaard \& Prock1, 2012).

One of the main results from the Licentiate thesis revealed the significant role of customers acting as an important starting point for integrating green thinking as well as adopting green initiatives within LSPs. In addition, there was a general perception among the LSPs studied in the Licentiate thesis that customer interest has increased and their green requirements have become more realistic over the years. However, as found both in the multiple case study in the Licentiate thesis and the case study in this dissertation, the degree of customers' green requirements seems to vary depending on factors such as type of industry, company size and the level of a sustainability profile. Furthermore, among the LSPs studied in the Licentiate thesis, customers were not only highlighted as a driver for the environmental work but also mentioned as a barrier. Reasons given for this outcome concerned the lack of customer support in the form of unwillingness to pay extra or share the cost of the investment in green 
solutions. Another reason given was the variety and different nuances as well as sometimes unspecific green requirements among customers. PN Logistics also mentions that the degree of customers' environmental knowledge and their environmental work is somehow correlated with how specific they are when formulating their environmental requirements. A possible explanation for customers' varying and sometimes low degree of environmental interest is given by Gammelgaard and Prockl (2012). The authors suggest that manufacturing companies have to date been focusing on their internal environmental work and have thus not considered environmental issues connected to their transport and logistics activities. Another reason mentioned by one of the case companies in the Licentiate thesis concerned customers' internal lack of communication, for example between the purchasing and environmental department, that may lead to misunderstandings, unreasonable requirements and a risk that some environmental issues are never discussed.

However, one way to overcome this communication or organisational barrier among customers can be illustrated in the PostNord case study by the initiative of Environmental Manager to Environmental Manager meetings. During these meetings, the customer is given the opportunity to describe their environmental expectations of PostNord as a sub-contractor and also express if PostNord lives up to these requirements or not. As mentioned previously, one of the purposes of these meetings is that the customer's environmental work is spread within its own company. By doing this, the sales force of PostNord finds it easier to take up a more environment-related discussion with the contact person at the customer company. In addition, the sales people find it therefore easier to sell green service offerings that can further improve the customer's environmental work, since it is not certain that the contact people at the customer company have green expertise or have any sense of what their group aims for regarding environmental issues. This example not only shows the relevance of spreading environmental knowledge but also provides the opportunity to better understand customers' green requirements. In addition to these meetings, different kinds of customer and competitor surveys exist in order to reveal customers' perceptions of PostNord's environmental performance. However, these surveys may not be sufficient as an input for developing green service offerings. Discussing and interacting with customers regarding environmental issues can thus act as a facilitator for LSPs to identify customers' sometimes latent needs and open up for opportunities to provide suggestions for green improvements. Not solely reacting to customers' green demands is also highlighted by Gammelgaard and Prockl (2012). The authors instead recommend LSPs to develop a more proactive approach for environmental initiatives in order to create cost savings and increased business for themselves.

To start integrating and collaborating with customers regarding environmental issues and thus develop and design green solutions for the supply chain is something that is highlighted by many researchers (e.g. Colicchia et al., 2013; Prockl \& Gammelgaard, 2012; Jumandi \& Zailani, 2010). As found both in the multiple case study in the Licentiate thesis and also in the PostNord case study, the interest among the LSPs studied revealed a fairly positive approach towards collaboration with customers regarding green initiatives. For example, one LSP from the multiple case study in the Licentiate thesis states that:

\section{"We want to have close cooperation with our customers, to be engaged, creative and solution-orientated and try new approaches and methodologies."}

Similar reasoning was shared by another LSP in the multiple case study in the statement:

\footnotetext{
"We must work together with our customers in order to get the best effect."
} 
These findings are in line with the result presented by Prockl and Gammelgaard (2012), who suggest that improvement of sustainability issues "cannot be accomplished by one actor alone" and the participating LSPs in the study state that they cooperate with their subcontractors and their customers in order to improve sustainability issues. However, despite the growing interest in collaboration regarding reducing environmental impact of the supply chain, Colicchia et al. (2013) argue that examples of such effective collaboration actions seem to be lacking.

As highlighted by Jumandi and Zailani (2010), both LSPs and their customers need to improve their transparency towards each other in order to use each other's capabilities and find innovative ways for green improvements. The desire for a more interactive collaboration regarding green initiatives can also be found in the case study in the Licentiate thesis, where some of the LSPs studied placed demands on their customers and wanted them to contribute as well. One of the LSP studied stated that:

\section{"Customers just want to listen and hear that we are working with green initiatives but do not want to be a part of it. We need customers that are willing to do something as well."}

Placing requirements on its customers regarding environmental issues can also be found in the PostNord case study, within the Mail Sweden business area and their Economic Climate service offerings. PostNord places high requirements on its customers which must be met in order to be able to buy these kinds of services.

The above discussion reveals that there seems to be an interest among LSPs in engaging with customers towards green initiatives. According to the result in Paper 3 in the Licentiate thesis, the LSPs studied could be categorised on the basis of whether they interact on green issues with all customers - the market in general or a specific customer segment with a green interest. However, in order to benefit from each other's skills and resources in developing green solutions, there seems to be a need for LSPs to find dedicated customers that share the same values and environmental attitude and beliefs. As mentioned by Wolf and Seuring (2010), improving environmental issues does not solely concern changing business practice within a single company; rather, it concerns changing attitudes across the complete supply chain.

\section{External knowledge and resources - other stakeholders}

However, as mentioned in the literature, customers are not the only external source to gain green knowledge and resources. In addition, as found among the LSPs studied in the Licentiate thesis, networking with other companies and organisations was seen as valuable input for ideas regarding their further environmental development. However, the degree to which this was done varied between the LSPs studied. As the result of Paper 3 revealed, some LSPs were more active in networking with competitors as well as others (such as authorities and research institutes) while other LSPs took a less active approach by staying aware of the surrounding environment.

Moreover, as is apparent from Table 8 (see Chapter 5) connected to the questionnaire survey results, it seems to be more common to cooperate with suppliers/partners than with customers in order to reach environmental targets. As illustrated in the PostNord case study, collaboration with suppliers can be a valuable source to integrate new green knowledge and push the environmental work further. PostNord tests almost everything on the market and since the company is a big actor on the market, PostNord is an interesting test partner for both 
Volvo and Scania. For example, tests have been done with vehicles computers to monitor drivers' behaviour, DME trucks and hybrid vehicles as well as methane cars.

However, collaboration with competitors was not something that was either specifically highlighted in the literature or from the different case studies. As found in the Licentiate thesis, competitors were not seen as a major driver for LSPs' adoption of green initiatives. There was a perception among some of the LSPs studied that their environmental work was ahead of their competitors and in order to maintain that position they strive to be aware of their competitors' green development. Similar thoughts can be found in the PostNord case study. None of the competitors is seen as an environmental leader; however, according to the Environmental Manager, the only difference might be that competitors have been better than PostNord at communicating their environmental performance. The findings regarding competitors as an untapped source of green knowledge as well as a potential collaboration partner is in line with the results of Colicchia et al. (2013). Their study revealed that collaboration with other actors in the logistics and transportation industry is less common; instead, collaboration with external organisations, foundations and institutions seems to be the most common source of external green knowledge.

Engagement in different networks was also mentioned by PostNord as a good source of input to the environmental work. For example, PN Logistics is engaged in the Green Corridors network as well as the KNEG (carbon neutral road transport) network. The latter is perceived as a good network, where other players in the transport and logistics industry, together with fuel producers as well as vehicle manufactures, are gathered in order to develop and show the need for alternative fuels. In line with the result of Colicchia et al. (2013), collaboration initiatives with research centres as well as universities have been identified in the PostNord case study. This finding concerns first and foremost PostNord's efforts regarding their city logistics concept. The aim of the network was to find further information and knowledge of the city logistics area and together with the expertise within PostNord create some kind of consensus.

To sum up, the discussion above indicates, in line with the result of Abareshi and Molla (2013), that in order to improve green performance on different levels, there may be a need for LSPs to adopt knowledge from different kinds of sources. The underlying reasons and need for using these different sources may not all fulfil the same purpose. For example, engagement in different kinds of networks and collaboration initiatives with suppliers can be seen as a way to improve an LSP's internal environmental performance by improving internal processes and procedures as well as gaining new green knowledge and resources. Meanwhile interacting with competitors and customers, collaborations have a more external and marketrelated focus on the environmental work. This may lead to improved communication of environmental efforts and increased understanding of customer's green demands, which enable LSPs to suggest, as well as develop, appropriate green solutions for the customer's specific supply chain. In addition, as the questionnaire survey results in Table 8 (see Chapter 5) reveal, the most common supply chain related measures adopted by LSPs to reduce the environmental impact have a more internal than external focus. Having a more internal focus on improving the environmental impact of its business activities seems like a natural point to start its green development. However, in order to respond to market demands, a more external focus regarding the environmental work should not be neglected for LSPs' further development of greening their business as well as service offerings. Furthermore, as mentioned in Paper 1, the green knowledge and resources needed are likely depending on the 
LSP's intents with its environmental work as well as developing of green services, and need therefore to be aligned with the strategic ambitions.

\subsubsection{IT use and expertise}

In general, adoption of different kinds of technology has been identified in the literature as a facilitator when developing logistics services but it has also been identified as an important tool when mitigating environmental efforts (e.g. Prockl \& Gammelgaard, 2012; Zailani et al., 2011). This is in line with the result in Paper 1 in the Licentiate thesis. For example, the result suggests that ICT can become an important facilitator when collecting, processing and displaying environmental aspects in LSPs' service offerings as well as acting as a tool in customisation and differentiation of the offering.

However, the results of the questionnaire survey as revealed in Table 12 (Chapter 5) regarding arguments for investing in ICT to support green initiatives, show that the most common and significantly strongest arguments are improved competitiveness and reduced cost of transport and logistics operations. It should be noted that the argument for improved internal control of environmental performance was singled out as the third most common argument, implying that this argument was not significantly stronger than the other arguments. Despite this, the LSPs studied in the Licentiate thesis mention that their ICT systems were not seen as being as supportive and flexible as the companies would like regarding green information. For example, inadequate IT systems were mentioned, especially with regard to estimating emissions. However, this information barrier was found in Paper 2 to have a lower impact among the small companies compared to the larger companies.

Even if technology is highlighted in the literature as a major facilitator for environmental efforts (Prockl \& Gammelgaard, 2012), the question remains to what extent ICT, for example, is adopted by LSPs in order to control and improve its environmental impact. For example, Table 11 (in Chapter 5) derived from the questionnaire survey, shows some examples of ICT applications for managing the environmental impact. The result from Table 11 reveals a relatively low mean in general among the suggested ICT applications, which can question the extent of ICT application adopted in order to manage the environmental impact. Similar results can be found in Paper 2 in the Licentiate thesis, where the results indicate that LSPs do not widely use technology to support green initiatives. Thus, as mentioned in section 5.2, there may be a hidden potential to integrate IT expertise (e.g. the IT department) to a larger extent in the environmental work and further in the development of green services among LSPs.

Furthermore, technology can also be seen to facilitate knowledge and network relationships (Gammelgaard et al., 2012). The relevance of ICT integration and synchronisation can be seen from both an internal and external perspective. From an external perspective, Table 13 (in Chapter 5) derived from the questionnaire survey, shows that ICT integration with customers seems to be most common among the studied supply chain actors. However, all the studied supply chain actors in Table 13 receive a relative low mean, which can suggest a great potential for improvements for LSPs within this area. It should be noted that this question was asked from a general perspective. It may, however, be reasonable to argue that integration of IT systems and adoption of ICT applications can be seen as a way to facilitate information sharing and improve network relationships from a green perspective as well. This reasoning may also be relevant from an internal perspective, especially for larger LSPs with different functions and global units within the organisation. 
Another issue connected to IT use and expertise concerns the transparency of green information. As suggested by Jumandi and Zailani (2010), both LSPs and their customers need to improve their transparency towards each other in order to effectively use each other's knowledge and resources to develop innovative approaches for environmental improvements. Similar reasoning can be found in the study by Gammelgaard et al. (2012), who mention lack of visibility as a reason for the hidden potential of a more proactive approach when developing green service offerings among LSPs.

The knowledge of the relevance and potential towards increased information transparency regarding environmental issues can be found in the PostNord study. As mentioned in the case study, PostNord tries to be as transparent as possible when it comes to communicating the company's environmental objectives to its customers. However, the Environmental Manager of PostNord states:

"From a general point of view, PostNord could become better at communicating all environmental work we actually do."

Improving the information transparency is something that PostNord works with and where it believes it has come a long way, in particular when it comes to informing customers of what kind of environmental initiatives and efforts have been made. For example, one on-going initiative is to mark which trucks are the DME cars (electric hybrids) with the aim of using these trucks as an "advertising pillar" for PostNord's environmental work.

As mentioned by the LSPs studied in the study by Prockl and Gammelgaard (2012), there seems to exist a desire and a need for increased transparency and a green communication that goes beyond the required environmental documentation. In addition, as the Environmental Manager of PostNord states:

\section{"...much of the work is about packaging and communicating what we already do. It cannot just be about carbon offsetting, there must be some significant value for the customers."}

Being a trustworthy actor and converting the promises given to customers into actions was also something that was mentioned by the LSPs studied in the Licentiate thesis. One LSP in the multiple case study expressed an overall fear of scaring customers off if there is a lack of entirely reliable data and information. Another LSP in the multiple case study mentioned that the first thing the company has to do is to start communicating the company's environmental policy and thereafter gradually do more. The company wants to be more confident before it starts to promote green service offerings since the company does not want to end up in a situation of launching a green service offering and then doing nothing about it. Instead, the company wants to build trust both within the company and towards their customers.

In addition, the importance of transparency in showing what the customers actually pay for regarding environmental aspects is expected by one LSP in the Licentiate thesis to increase in the future. Regarding visualising pricing for regular service offerings, the company has a tool on the website where customers can fill in their own data such as destination and additional costs. It is possible to choose between different transport options and it is easy to understand. In the future, the company also aims to integrate sustainability aspects connected to the service offerings. Not only will customers then become more aware of the sustainability impacts of their transports, but it can also make it easier for the company to communicate its sustainability work with its customers, something that is lacking today. 
To sum up, even if technology is identified in the literature as a facilitator to control and improve the environmental impact, the adoption of ICT applications appears not to be widely used among the studied LSPs in order to support green initiatives. For example, the existing ICT systems were not perceived to be as supportive and flexible as the LSPs studied wished for in order to handle environmental information. However, the importance of ICT was nevertheless highlighted both in the multiple case study in the Licentiate thesis and in the PostNord case study. The analysis above indicates that an increased focus on information transparency regarding green initiatives can be utilised in order to; build trust both within the own company and towards customers; increase the external communication of adopted green initiatives; as well as act as a way to visualise the environmental impact of customers' transports. The latter one can be especially relevant for LSPs development of green service offerings in order to demonstrate the potentially achieved environmental improvements to their customers. 
- 178 - 


\section{CHAPTER}

\section{CONCLUSIONS AND DISCUSSION}

This chapter presents the main conclusions of this research with the aim of answering the purpose of this dissertation. The chapter begins with a presentation of the developed NSD framework followed by a concluding discussion of the different NSD dimensions. Thereafter, follows a concluding discussion of the developed framework from a green service development perspective. In addition, this chapter also involves a section that presents some recommendations for LSPs for how to interpret and address the results found in this research. The chapter then continues with a discussion regarding both theoretical contributions as well as managerial implications of the findings from this research. Finally, this chapter ends with a section presenting some recommendations for further research. 
-180 - 


\subsection{The conclusions of the research}

The conclusions in this thesis can be described as twofold. The first part, the LSP's general NSD framework with its including dimensions and underlying pre-requisites, aims to answer RQ1 and its sub-questions. The second part, which aims to answer RQ2, concerns the discussion and analysis of developing green service offerings among LSPs. The discussion and analysis of the second part is structured and related to the developed NSD framework in the first part.

\subsubsection{The LSP NSD framework}

The analysis model in this thesis, developed in Chapter 3, has through a step-wise approach been further developed and refined through analysis of theoretical and empirical data into an LSP context. The developed LSP's NSD framework, with its identified dimensions and underlying pre-requisites is illustrated in Figure 8.1 below.

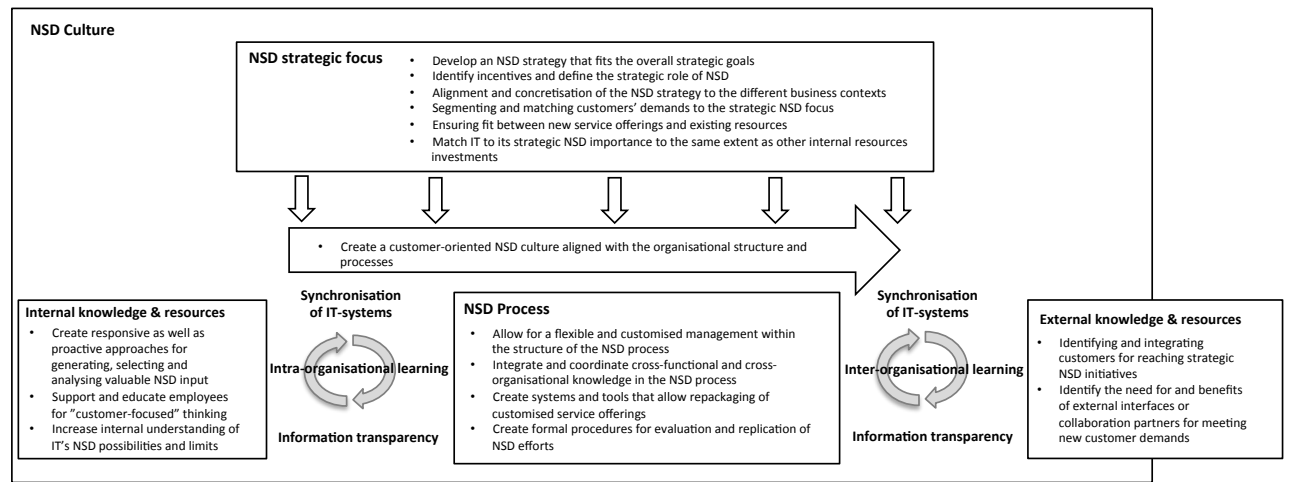

Figure 8.1 The NSD framework for LSPs.

The developed framework presents a holistic view of LSPs' NSD efforts by revealing different dimensions and pre-requisites to take into consideration in the development of new service offerings. As identified in the analysis, each NSD dimension has its own role and each provides specific understanding of LSPs' NSD. In addition, as found in section 7.3, the different NSD dimensions have a certain connection with and influence on each other. Thus, the different NSD dimensions should not solely be viewed as isolated dimensions; instead, there is a need for LSPs to have a holistic view and understanding of NSD activities' reciprocity. The findings in the thesis confirm, therefore, current research which highlights that achieving NSD success is a result of a more comprehensive approach which manages to coordinate a large number of aspects competently and in a balanced manner (e.g. Menor \& Roth, 2008; Ordanini \& Maglio, 2009; Johne \& Storey, 1998). Moreover, as stated in the analysis, the link between and the focus on the different NSD dimensions seem to be influenced by the company's strategic NSD focus. In addition, factors such as size of the company as well as level of newness and scope of the developed service offering may also influence the link and relevance of the different NSD dimensions.

In this dissertation, five different NSD dimensions have been identified in the literature and further refined into an LSP context. A brief description of their roles and content is presented below. 


\section{- A customer-oriented and learning-focused NSD culture}

The role of an LSP's NSD culture is to provide a basis for the company's ability and attitude towards the development of new service offerings. This is in line with Flint et al. (2005), who describe it as a foundation for managing and supporting an LSP's NSD activities. The results from the analysis in this dissertation indicate that LSPs can achieve NSD benefits by striving towards a more customer-oriented and learning-focused NSD culture. For example, operating in markets that are typified by variations in customer's characteristics and increasing customer demands, as LSPs do, raises a need to better understand the customers. The result can be supported by Wallenburg's (2009) argument that whether the development of new service offerings will be perceived as successful or not will depend to a large extent on an LSP's knowledge of what their customers value. Being more customer-oriented and providing more customised logistics solutions to its customers needs thus, as discussed in the analysis, to somehow be reflected in the NSD culture and also in the NSD activities of LSPs. As a consequence, LSPs need to consider how to motivate and support employees' attitudes towards a more customer-oriented thinking and acting and the prevalence of customer interaction as well as integration regarding NSD efforts within their own company.

Furthermore, the results in the analysis suggest LSPs should move towards the establishment of learning NSD cultures in order to take advantage of existing knowledge and resources regarding their NSD efforts. The trend regarding changing customer behaviour towards more customised and complex logistics solutions (e.g. Third-Party Logistics Study, 2012; PostNord Annual Report, 2011), favours those LSPs who succeed in creating a cross-functional and flexible NSD culture based on their knowledge and resource base in order to manage parallel logistics solutions to various customer and market segments. In addition, as suggested in the analysis, an LSP's NSD culture should not solely consider an internal focus but also an external focus that promotes and supports learning from other actors (e.g. customers and suppliers). In line with the reasoning of Flint et al., (2008), this may lead to better dialogues and finding better solutions to serve e.g. customers in the supply chain.

Depending on the scope and level of newness of the service initiatives, this may require collaboration and coordination of different functions as well as different actors and it is easier to manage when such initiatives are endorsed from the top. The relevance of managerial support and engagement in order to realise such an NSD culture is supported in the literature by several researchers (e.g. Busse \& Wagner, 2008b; Panayides, 2007; Flint et al., 2005).

\section{- Define NSD strategic focus and its alignment with business goals and resources}

As described in the analysis, the strategic focus on NSD has a central role among all the different activities related to NSD. In order to keep focus on the NSD efforts, the findings suggest that LSPs should identify incentives and define the strategic role of their NSD efforts. This is in line with the argument of Sonio et al. (2012), regarding the need for LSPs to understand the options for expanding their business scope. Thus, the results from the analysis stress the relevance for LSPs to fit their NSD strategy to the overall business goals as well as to existing resources. For example, as mentioned in the analysis, alignment with other strategies and the company's image can support reaching set strategic goals as well as facilitate both internal and external communication. At the same time, the results regarding a fit to existing resources concerned first and foremost gaining efficiency in their own production mechanisms as well as minimising the existence of 
overlapping service offerings, which can result in high cost and an unclear picture of the service offerings to the customers.

Moreover, since many large LSPs have a broad range of service offerings in their product portfolio and operate in different markets, the results from the analysis, in line with Busse and Wagner (2008b), also suggest that LSPs need to consider alignment and concretisation of the NSD strategy to the different business context. As mentioned in the analysis, different business units within the company may face different market conditions in the form of customers' requirements and preferences, and have different resource requirements for their NSD efforts as well as aiming for different market positions.

The alignment to the business context and existing resources can also be a way for LSPs to identify and ensure a comprehensive resource base for NSD efforts, or whether there is a need for adjustment or modification of existing resources and facilities. One example of such a resource found in the case study concerns IT resources. Thus, the results of the analysis indicate a more long-term and proactive perspective when LSPs evaluate their IT and other internal resource investments in order to ensure that it supports their NSD efforts as well as matching market expectations.

Furthermore, the analysis identified not only the relevance of an internal focus on an LSP's NSD strategy but also an external link to, for example, customers' expectations and demands. Due to customers' changing and various demands it may be relevant to argue that the expression "one solution fits all" does not apply to LSPs. Therefore, in line with Speh (2008), to fully benefit from their NSD efforts and develop appropriate service offerings, it is recommended that LSPs increase their market understanding and consider segmenting and matching customers' demands to their strategic NSD focus.

\section{- Existence of structured and flexible NSD processes and structures}

Based on the discussion in the analysis, the role of LSPs' NSD processes can be described as a fundamental structure to enable and facilitate the management, evaluation, learning and improvement of the included steps and activities. However, the relevance of allowing a more flexible and customised management of the NSD process was identified in both the literature and the case study. The findings suggest that LSPs should emphasise the fact that different NSD solutions or initiatives may require different levels of resources and formalisation and thus stress the need for processes that are adapted and optimised to a specific situation.

Further, the results in the analysis also reveal the relevance for LSPs of integrating and coordinating cross-functional as well as cross-organisational knowledge in the NSD process. These results are in line with Chapman et al. (2003), who stress that LSPs should focus on coordination of activities among partners in the network in order to gain and integrate new knowledge into the NSD process. Another essential issue mentioned in the literature regarding NSD processes concerns the existence of feedback loops, which is seen as critical in order to facilitate analysis of the intangible aspects of the service design (Johnson et al., 2000). Thus, as a consequence of the above discussion, in broadening the scope of integration of both internal and external knowledge, the results point to the relevance for LSPs of establishing formal procedures for gathering lessons learned from specific NSD projects as well as ensuring replication of successful NSD efforts. 
One further aspect found regarding NSD processes concerns the dilemma between standardised and customised service offerings. It may not be advantageous in the long run for LSPs to choose only one approach, due to increasing demands for customised logistics solutions together with the LSP industry's strong focus on economies of scale. Thus, the result indicates a need for LSPs to create systems and tools for reusing knowledge and resources dedicated to a specific customer for a broader customer base.

\section{- Identify and integrate both internal and external NSD knowledge and resources}

Regarding the role of NSD knowledge and resources, this is described in the analysis as the kernel that feeds and forces the development of new service offerings further. Even though many researchers stress the importance of identifying and responding to market needs (e.g. Alrubaiee, 2013; Ordanini \& Maglio, 2009; Menor \& Roth, 2007), the LSP industry is described in the literature as having a rather low degree of monitoring the market environment, and is also perceived to be more reactive than proactive in its NSD efforts. However, this research indicates that LSPs may benefit from creating more responsive and proactive approaches for generating and analysing valuable NSD input. These approaches should both include a short-time perspective, by responding to customers current needs, and have a more long-term perspective and provide possibilities to reveal customers' latent needs and new market possibilities.

Further, one interesting point based on the literature findings, as well as in the case study, is that there seems to be a shift in focus among LSPs from being asset-based to being a more skills and knowledge-based organisation. This is in line with Jumandi \& Zailani (2011) highlighting the important role of employees among LSPs as well as the need for more multi-skilled generalists than technical-oriented experts in order to respond to the competitive and complex business environment of LSPs. As identified in the analysis, the results of this research suggest that LSPs should support and educate employees for a more "customer-focused" thinking as well as increase internal understanding of IT's NSD possibilities and limits.

The result in this thesis also emphasises the external need for identifying and integrating NSD knowledge and resources. It is recommended that LSPs identify and integrate customers in order to reach strategic NSD initiatives, but also identify the need and benefits of external interfaces or collaboration partners to meet customer demand, which require knowledge and skills that may not exist within their own company.

\section{- Improve IT synchronisation and information transparency}

As identified in the analysis, the role of IT can be described as twofold. Firstly, through its role as a critical resource in the design and production phase of LSPs' service offerings, as well as its importance as a value-adding component. Secondly, by its role as a coordinator and integrator in order to make creative use of information and ensure flexibility and efficiency of relationships and networks in the supply chain regarding NSD efforts. In this dissertation, the latter has received a greater focus in the developed NSD framework. The findings in the analysis do not reveal any major differences from current research regarding IT importance in the development of new service offerings (e.g. Menor \& Roth, 2007; de Jong \& Vermuelen, 2003). However, the results indicate the relevance for LSPs of focusing on enabling synchronisation of IT systems as well as on information transparency in order to improve their NSD efforts. As identified in the analysis, benefits may be perceived both from an internal and external perspective. 


\subsubsection{The NSD framework from a green perspective}

This section aims to answer RQ2, which concerns the discussion and analysis of developing green service offerings among LSPs. The discussion in the area of Green Logistics regarding benefits achieved and reasons why LSPs should start to adopt green initiatives (partly addressed in the Licentiate thesis) has gradually shifted into what and how greening of LSPs business can be done (e.g. Colicchia et al., 2013; Rossi et al., 2013). An example of such a discussion topic, addressed in this research, concerns the development of green service offerings among LSPs. Despite the identification as a promising and fruitful area for both academia and managerial practice (e.g. Jumandi \& Zailani, 2010; Ho et al., 2009), the amount of research regarding this topic is still rather low. In addition, Gammelgaard et al., (2012) question whether development of green service offerings is a hidden and unseen potential among some LSPs.

However, the results of this research reveal some pre-requisites relevant for LSPs to consider in their efforts to develop green service offerings.

\section{- Creating green awareness in the NSD culture}

One finding in the analysis, as identified both in the literature and in the empirical data, concerns encouragement of participation and commitment regarding green initiatives within the organisation, for example the Climate Fund in the PostNord case. The fact that a culture that addresses green awareness and supports long-term thinking has a positive effect on LSPs general adoption of green initiatives as well as increasing the degree of green innovations efforts to be realised is also supported in the literature (e.g. Lin \& Ho, 2011; Zailani et al., 2011). Further, defining a "common picture and language" of the environmental work as well as development of green service offerings can facilitate collaboration efforts and knowledge exchange regarding green expertise within the company.

The research also stresses, in line with Abareshi and Molla (2013) and Zailani et al. (2011), among others, the relevance of top management support, whose engagement can influence the adoption of green initiatives as well as the existence of cross-functional green involvement within LSPs.

\section{- Defining the strategic approach of green service offerings}

Regarding the strategic focus, the result of this research shows that existence of green concerns in the overall business strategy is positively related to implementation of green initiatives within LSPs. In addition, as revealed in the analysis, integration of green concerns in the brand can also have an influence on increased green considerations in an LSP's operations as well as service offerings. However, as mentioned in the analysis, the incentives regarding the environmental work in general and development of green service offerings may vary. Thus, the findings indicate the need for LSPs to define the strategic role and incentives for developing green service offerings in order to keep a focus on NSD efforts.

Furthermore, as suggested in the analysis, an LSP's environmental work in general can be seen as a resource platform or toolbox for its development of green service offerings. In addition, as indicated in the analysis, the content and the precondition of creating such a platform may vary between different LSPs as well as within each company. Thus, the result in this research suggests that LSPs need to adapt their green NSD efforts to the different business contexts and market possibilities (e.g. customer requirements, resources 
and technological development available on the market) in order to match existing resources and skills with customers' green requirements.

Another finding concerns the perception of customers' various green needs and requirements, which consequently highlights a need among LSPs for segmentation of customers' environmental work and ambitions in order to increase the knowledge and understanding of customers' green attitudes and requirements.

\section{- Create processes and routines to facilitate spreading of green knowledge}

Due to the early phase of developing green service offerings, the results of the analysis identified aspects that can have a more indirect than direct influence on the development process of green service offerings. In addition to efforts to improve existing processes in order to decrease the environmental impact on the operations, the results of this research indicate the relevance of a process focus for spreading green knowledge both from an external and internal perspective. From an external perspective, adoption of certifications, standards (e.g. ISO 14001) as well as procedures for environmental calculation and documentation can act as a way to increase transparency and credibility of LSPs' environmental work as well as to green service offerings. In addition, the results of this research are in line with Abareshi and Molla (2013), by stressing the need for an internal focus on developing processes and routines in order to facilitate the spreading and integrating of green knowledge among employees as well as identifying customers' green requirements.

\section{- Improve green internal knowledge and build green collaborations}

The result in this research shows the relevance of gaining and integrating both internal and external knowledge regarding LSPs' environmental work and further development of green service offerings. Regarding internal knowledge, LSP's employees are identified in the analysis as important for implementing and realising the company's environmental goals and are thus seen as co-creators of green value. Thus, providing training and education in order to increase the level of green awareness and knowledge among the LSPs studied is seen as important. In line with Maack's (2012) results, this research stresses the essential role of sales people's green knowledge as an important link between customers and their attitude towards green service offerings. Further, the results from the analysis also suggest LSPs should improve the generation of existing/previous knowledge and skills regarding green initiatives within the company, which can constitute a basis for ideas regarding development of green service offerings.

As revealed in the analysis, there seems to be a strong desire among the LSPs studied to integrate and collaborate with customers regarding green initiatives. Further, due to the sometimes varying and unrealistic green requirements of customers, the result of this research reveals the relevance of educating and spreading green information among customers in order to identify latent needs and find different ways to meet those demands. Furthermore, the findings, in line with Colicchia et al. (2013), show that the existence and degree of collaboration and networking with other companies and organisations seems to vary between the LSPs studied.

However the results in this research suggest LSPs should operate on a more system-wide basis and collaborate across conventional boundaries, both internal and external, in order to utilise each other's knowledge and resources towards the development of green service offerings/solutions in the supply chain. 


\section{- Increase transparency of green information both internally and externally}

Regarding the NSD dimension IT use and expertise in the context of developing green service offerings, the results of the analysis reveal a strong focus on the transparency of green information. Identified arguments regarding its importance concerned, among other things, building both internal and external trust regarding the environmental work, revealing what customers are actually paying for regarding environmental aspects, as well as increasing possibilities to effectively use other actors' knowledge and resources in the supply chain in order to develop innovative approaches for environmental improvements. Further, the relevance of increased information transparency regarding green aspects is expected to increase in the future; similar reasoning can be found in the study by Prockl and Gammelgaard (2012).

In addition, the results in this research also stress the need for LSPs to evaluate the effect of IT's role in supporting green initiatives and green service offerings. As mentioned in the analysis, IT can facilitate the internal control of environmental performance, information sharing and network relationships regarding green initiatives. However, the results indicate the existence of inadequate IT systems and a general low level of using technology when supporting green initiatives within the studied LSPs. Despite this, the results of this research point to the relevance of integrating IT expertise and synchronisations of IT systems in order to facilitate and support environmental work and development of green service offerings among LSPs.

To conclude and reflect upon the results from the research in this dissertation: some possible explanation factors have been identified which to some degree can help to understand the difference between LSPs' general NSD efforts and the development of green service offerings. One possible explanation factor concerns the strategic focus of LSPs' environmental ambitions, which in turn can be seen as a relatively critical decision point for whether LSPs choose to develop any green service offerings compared to general NSD efforts where new services per definition need to be developed. In addition, the existing environmental work can also explain the difference since it can be viewed as a resource base and thus create the conditions and influence the development of green service offerings. Another explanation factor that may have a significant impact in explaining the differences concerns the fact that environmental considerations are not seen as a natural core competence among LSPs. This in turn may lead to higher requirements, compared to NSD efforts in general, to increase the internal knowledge and skills, as well as collaboration initiatives with other actors, in order to obtain an exchange of knowledge and resources related to environmental issues.

\subsection{Recommendations for LSPs towards the findings}

As mentioned in the introduction to this chapter, this section aims to synthesise and bundle the results from section 8.1.1 and 8.1.2 discussed above. The results from these sections should not be viewed as entirely separate parts; instead, this section aims to provide some recommendations for LSPs regarding how they can interpret and address the results presented in this research. The recommendations provided can thus be seen as a complement to the developed NSD framework. In other words, this section can be considered to act as a bridge between the results found in this research and practical implications for LSPs. 
As a start, one might ask why it is important for LSPs to focus on improving their NSD efforts as well as paying attention to green aspects into this process. As mentioned earlier in this thesis, the fact that the LSP industry is affected by, among other things, increasing competition and internationalisation puts pressure on the ability of LSPs to add value to shippers' bottom lines (Third-Party Logistics Study, 2012; Flint et al., 2005) as well as to improve growth strategies such as taking market shares from competitors and strengthening existing customer relationships (Wagner \& Franklin, 2008). Thus, one way to increase the margins and realise the growth strategies for LSPs is to focus on their services and the development of these.

In addition, due to the focus regarding environmental impact, sustainability awareness has become an essential part of a business's operations (Prockl \& Gammelgaard, 2008). This has led to increasing pressures on LSPs regarding how they respond to, manage and profile themselves towards environmental concerns, which can, in turn, become a question of differentiating. In general, it is suggested that brand maintenance issues are particularly applicable in the LSP industry, where several actors offer more or less the same services (Lieb \& Lieb, 2010). Therefore, greening of the business, as well as branding, can be seen as a way for LSPs to differentiate both their business and their service offerings from competitors.

Based on the findings of this research, some stages that can help LSPs on the path to improving their NSD and integrating green concerns into this process are presented below.

\section{STAGE 1: Create a general NSD foundation}

The first step LSPs need to take is to create a solid foundation for their NSD efforts. This step involves setting the goals and creating the right conditions for the NSD efforts, and can be linked to an LSP's NSD strategy, culture and process focus. For example, the strategic NSD objectives need to be aligned to the company's different business contexts and resources in order to identify a need for the adjustment of existing resources and facilities, in particular IT resources. In addition, this stage also involves the creation of an increased customer and market understanding. To fully benefit from the company's knowledge and resources, LSPs should strive to create a cross-functional and flexible NSD culture relying on defined processes and structures in order to manage parallel logistics solutions to various customer and market segments.

\section{STAGE 2: Integrate green aspects in this foundation}

The next stage includes first and foremost defining the strategic approach of an LSP's green service offerings as well as creating green awareness and involvement among employees. It also includes the need for LSPs to adjust their green NSD efforts to the different business context and market possibilities, as well as increase the knowledge and understanding of customers' green attitudes and requirements. In addition, LSPs may also focus on creating routines and processes to facilitate the managing and spreading of green information and knowledge.

The next two stages are relevant both from a general and a green NSD perspective.

\section{STAGE 3: Focus on feedback, learning and replication to improve the NSD process}

The third stage focuses on the ability to maintain and improve NSD knowledge within the company as well as to take advantage of lessons learned based on previous NSD activities and projects. LSPs can provide employees with education and training in order to improve internal NSD knowledge within areas such as a more customer-focused thinking, internal 
understanding of IT's NSD possibilities as well as green awareness. To fully benefit from existing, as well as integration of new, NSD knowledge, this stage advocates a certain focus on feedback loops and replication of successful NSD efforts. In addition, this stage also includes LSPs' ability to reuse knowledge and resources dedicated for a specific customer to a broader customer base.

\section{STAGE 4: Integrate new knowledge by external interaction}

The last stage has a more external focus and includes identifying and involving of relevant and appropriate stakeholders (e.g. customers, suppliers) in order to gain and integrate new knowledge into the NSD process. However, this stage requires a certain coordination of activities among the involved actors, support and commitment from the top management, as well as increased information transparency through the synchronisation of IT systems.

\subsection{Theoretical contribution}

As previously mentioned in section 1.6, the contributions of this research can, from a theoretical perspective, be seen as a way to link the logistics and service development research areas together. The developed NSD framework and its included dimensions as well as identified pre-requisites offer support in giving a deeper understanding of LSP's NSD as well as implications for developing green service offerings. Through the latter, the findings of this research also make a contribution to the green logistics research field in the specific context of LSPs.

Adopting and integrating the NSD perspective and combining it with existing research regarding LSPs service development can, from one point of view, be seen as a contribution in itself to the logistics field, due to the fact that the existence of NSD research within the research field of LSPs is new and is beginning to emerge (e.g. Wagner \& Sutter, 2012; Busse, 2010; Flint et al., 2005). Therefore, the contributions of this research have been to combine and extend insights from previous studies, which resulted in an NSD framework aiming to provide a holistic view of essential NSD dimensions as well as pre-requisites relevant to consider when studying LSPs' NSD efforts.

In addition, the developed NSD framework and its adaptation from an LSP perspective can be seen as further building on the research of Menor and Roth $(2007,2008)$ and thus also contribute to the service development research. The findings from this dissertation can partly be seen as a response to many researchers' requests for more conceptual and empirical NSD research, as well as key strategic factors influencing NSD performance (Edvardsson et al., 2013; Page \& Schirr, 2008; Froehle \& Roth, 2007). Further, the focus of much NSD research has concerned the financial service organisations and more recently high-tech service firms (Papastathopoulou \& Hultink, 2012). Thus the adaptation to an LSP setting in this dissertation can contribute with increased understanding of another service sector worth considering.

Based on the findings and the contributions from the Licentiate thesis regarding LSPs' adoption of green initiatives and how these can be reflected in the service offering, this dissertation provides further understanding of pre-requisites relevant for LSPs to consider when integrating green concerns in their NSD efforts. Due to its early phase as a research stream, especially in the context of LSPs, the results of this research can be seen as an early contribution to the green logistics research by creating a foundation, which highlights relevant dimensions and pre-requisites needed towards LSPs green service development. For each 
dimension, this research confirms previous research but also extends with empirical findings. In addition, the findings can also provide valuable insights to the general green product/service development research within the service development area.

\subsection{Managerial implications}

Contributing new insights and knowledge regarding NSD within the LSP industry is not only relevant from an academic perspective but also from a managerial and practical perspective. Thus, the results from this research can offer guidance and insights for essential dimensions and pre-requisitess to better understand and practise NSD within the company.

As mentioned by Wagner and Franklin (2008), without a general system or structure for NSD activities, LSPs will not be able to benefit to the fullest extent from these efforts. From a general NSD perspective, the framework developed in this research can help LSP managers to maintain a more holistic perspective on NSD. The identification of the different NSD dimensions and their underlying pre-requisites can help LSPs managers to understand what issues need to be addressed and thus provide inspiration for their continued efforts to improve NSD efforts. Further, based on the general NSD framework, the results of this research identify and discuss several pre-requisites when developing green service offerings.

It is hoped that LSP managers will find the developed NSD framework as a strategic tool to use in the service development of the company in that it can help in evaluating and reaching a deeper insight into their own NSD activities and efforts and their relation to the company's NSD goals and strategy. Furthermore, regarding LSPs' green initiatives and reflection in the service offerings, LSP managers may view the result of this dissertation with a certain degree of recognition and see the results as a source of inspiration regarding integrating green concerns in the NSD activities and their service offerings. Even though earlier findings point to the infancy phase of developing green service offerings (Isaksson \& Huge-Brodin, 2013), the results from this research can create a further awareness and thus inspire forward-looking LSP managers regarding developing green service offerings.

Further, the research in this dissertation may be of particular practice for medium-sized and large LSPs that have an established service development function and a strong focus on improving NSD efforts, and want to address environmental issues, giving particular attention to the development of green service offerings. Although the developed NSD framework and results from this research cannot be generalised to be valid for all types of LSPs, the research focus of this dissertation as well as some of its results may nevertheless create awareness and act as a stimulus among other LSP players to consider the role of NSD and thus provide more value-added service offerings to their customers. Furthermore, the findings from this dissertation can also increase the knowledge among other actors in the supply chain (e.g. customers and suppliers) regarding their role in and contributions to LSPs' NSD efforts, not least in the development of green service offerings.

\subsection{Further research suggestions}

Finally, in this section, based on the findings from this research, some directions for further research are discussed. The findings and the developed NSD framework together with its results regarding green service development of this research can, from one point of view, be 
regarded as a platform from which more detailed research may be conducted, both from a theoretical and an empirical perspective.

In the development of the NSD framework in this thesis, five different NSD dimensions were identified and further explored. As mentioned in the conclusions, each of the different NSD dimensions has its own role and provides an understanding of LSP's NSD. However, since these dimensions have in this thesis been studied from a more strategic and overall perspective, it would be interesting to further investigate these dimensions per se from a more tactical and operational level. This can be done for LSP's service development in general but also when integrating green awareness into the service development.

Moreover, as mentioned in the literature, it is important to understand the fit between different NSD practices and activities in order to increase a company's ability to effectively develop new service offerings (e.g. Menor \& Roth, 2008; Ordanini \& Maglio, 2009; Lusch et al., 2007). The relevance of the link between the NSD dimensions studied has partly been explained and investigated in this study; however, due to the methodological choices in this dissertation, it is not possible to make any generalised assumptions of the LSP industry. Therefore, it would be interesting to conduct a survey study of a larger sample of LSPs and further develop the NSD dimensions studied as well as the identified pre-requisites into quantifiable parameters and investigate their relation to each other. Depending on the sample of LSPs, it would be interesting to investigate whether there are any differences between different subsets of LSPs.

Based on the findings from this research regarding the role of integrating new knowledge by external interactions both from a general and green NSD perspective, it would be interesting to expand the LSP perspective and include input from other supply chain actors, such as customers and suppliers. This would improve the richness of the findings relating to the relevance of integrating external knowledge and thus increase the insights into how these actors can make use of each other's knowledge and resources, especially in the context of green service offerings. A focus on relationships/network innovation for green initiatives is, according to Rossi et al., (2013), an area that is neglected in the existing literature.

Finally, another interesting topic identified in this research concerns the role branding might have in customer perceptions of an LSP's green credibility and image. As indicated both in the Licentiate thesis and in this dissertation, adopting green initiatives is seen as a way for an LSP company to improve its brand image. Due to high competitive pressure and an increasing number of new actors, integrating green concerns into an LSP's brand can be a way to differentiate itself from other competitors. Therefore, it would be interesting to investigate in what way an LSP's attempt to green the brand influences customer perceptions of the company's environmental work and service offerings. However, as an early step in this process of "greening" the companies operations as well as service offerings, LSPs must take a strategic decision regarding which brand level they want to change. Do they want to create a fully "green brand" or simply create a green profile of the company identity? It might be necessary to decide this in order to develop an appropriate brand strategy regarding not only how to manage and implement it within the company but also how to build trust with different stakeholders in the company. 


$$
\text { - } 192 \text { - }
$$




\section{REFERENCES}

Abareshi, A. \& Molla, A. (2013), "Greening logistics and its impact on environmental performance: an absorptive capacity perspective", International Journal of Logistics: Research and Application, Vol. 16, No. 3, pp. 209-226.

Alam, I. \& Perry, C. (2002), "A customer-oriented new service development process", Journal of Services Marketing, Vol. 16, No. 6, pp. 515-534.

Alrubaiee, L. (2013), "An investigation on the Relationship between New Service Development, Market Orientation and Marketing Performance", European Journal of Business and Management, Vol. 5, No. 5, pp. 1-26.

Arbnor, I. \& Bjerke, B. (1997), Methodology for Creating Business Knowledge, SAGE Publications Inc, Thousand Oaks, CA, US.

Avison, D., Jones, J., Powell, P. \& Wilson, D. (2004), "Using and validating the strategic alignment model", Journal of Strategic Information Systems, Vol. 13, No. 3, pp. 223-246.

Bellingkrodt, S. \& Wallenburg, C.M. (2013), "The Role of External Relationships for LSP Innovativeness: A Contingency Approach”, Journal of Business Logistics, Vol. 34, No. 3, pp. 209-221.

Bryman, A. \& Bell, E. (2011), Business Research Methods, $3^{\text {rd }}$ ed., Oxford University press Inc, New Yord, US.

Busse, C. (2010), "A procedure for secondary data analysis: Innovation by Logistics Service Providers”, Journal of Supply Chain Management, Vol. 46, No. 4, pp. 44-58.

Busse, C. \& Wagner, S.M. (2008a), An Audit Tool for Innovation Processes of Logistics Service Providers, in Wagner \& Busse (eds.) Managing Innovation - The New Competitive Edge for Logistics Service Providers, ${ }^{\text {st }}$ ed., Haupt Verlag, Berne, Switzerland, pp.107-134.

Busse, C. \& Wagner, S.M. (2008b), Implementing an Innovation Management System at a Logistics Service Provider - A conceptual Guideline, in Wagner \& Busse (eds.) Managing Innovation - The New Competitive Edge for Logistics Service Providers, $1^{\text {st }}$ ed., Haupt Verlag, Berne, Switzerland, pp.187-193.

Busse, C. \& Wallenburg, C.M. (2013), "Firm-level innovation management at logistics service providers: an exploration", International Journal of Logistics Research and Applications: A leading Journal of Supply Chain Management, Published online 23 Dec 2013, pp. 1-24.

Busse, C. \& Wallenburg, C.M. (2011), "Innovation management of logistics service providers - Foundations, review and research agenda", International Journal of Physical Distribution and Logistics Management, Vol.41, No. 2, pp. 187-218. 
Chapman, R.L., Soosay, C. \& Kandampully, J. (2003), "Innovation in logistics services and the new business model - A conceptual framework", International Journal of Physical Distribution \& Logistics Management, Vol. 33, No. 7, pp. 630-650.

Chase, R.B. \& Apte, U.M. (2007), “A history in service operations: What's the big idea?”, Journal of Operations Management, Vol. 25, No. 2, pp. 375-386.

Colicchia, C., Marchet, G., Melacini, M. \& Preotti, S. (2013), "Building environmental sustainability: empirical evidence from Logistics Service Providers", Journal of Cleaner Production, Vol. 59, pp. 197-209.

Corbin, J. \& Strauss A. (2008), Basics of Qualitative research: techniques and procedures for developing grounded theory, $3^{\text {rd }}$ ed., SAGE Publications Inc, Thousands Oak, California, US.

Cowell, D.W. (1988), "New Service Development", Journal of Marketing Management, Vol. 3, No. 3, pp. 296-312.

Creswell, J. W. (2009), Research design - Qualitative, Quantitative, and Mixed Methods Approaches, $3^{\text {rd }}$ ed., SAGE Publications, Thousand Oaks, California, US.

Cui, L., Shong-Lee, I.S. \& Hertz, S. (2009), "How Do Regional Third-Party Logistics Firms Innovate? A Cross-case-regional Study”, Transportation Journal, Summer, pp. 44-50.

Daugherty, P.J., Chen, H. \& Ferrin, B.G. (2011), "Organizational structure and logistics service innovation", The International Journal of Logistics Management, Vol. 22, No. 1, pp. 26-51.

Da Mota Pedrosa, A. (2012), "Customer Integration during Innovation Development: An Exploratory Study in the Logistics Service Industry", Creativity and Innovation Management, Vol. 21, No. 3., pp. 263-276.

DB Schenker (2014), Sustainability - Homepage, [Online], Available: www.logistics.dbschenker.se/log-se-en/environment_quality, Accessed, $12^{\text {th }}$ April, 2014.

de Brentani, U. (2001), "Innovative versus Incremental New Business Services: Different Keys for Achieving Success”, Journal of Product Innovation Management, Vol. 18, No. 3, pp. 169-187.

de Brentani, U. (1991), "Success factors in Developing New Business Services", European Journal of Marketing, Vol. 25, No. 2, pp. 33-59.

de Jong, J.P.J. \& Vermeulen, P.A.M. (2003), "Organizing successful new service development: a literature review”, Management Decision, Vol. 41, No. 9, pp. 844-858.

de Souza, R. \& Goh, M. (2008), Innovation in Outsourced Logistics Services in Asia - The LSP Dilemma, in Wagner \& Busse (eds.) Managing Innovation - The New Competitive Edge for Logistics Service Providers, $1^{\text {st }}$ ed., Haupt Verlag, Berne, Switzerland, pp.61-78.

DHL (2014), Green solutions - Homepage, [Online], Available: www.dhl.se/sv/om_dhl/grona_losningar.html, Accessed, 12 $2^{\text {th }}$ April, 2014. 
DHL (2010), Delivering Tomorrow - Towards Sustainble Logistics, [Online], Available: http://www.dpdhl.com/en/logistics_around_us/future_studies/sustainable_logistics.html, Accessed: April $2^{\text {nd }} 2014$.

Easingwood, C.J. (1986), "New Product Development for Service Companies", Journal of Product Innovation Management, Vol. 3, No. 4, pp. 264-275.

Edvardsson, B., Meiren, T., Schäfer, A. \& Witell, L. (2013), "Having a strategy for new service development - does it really matter?", Journal of Service Management, Vol. 24, No. 1, pp. 25-44.

Eisenhardt, K. (1989), "Building Theories from Case Study Research", Academy of Management Review, Vol. 14, No.4, pp. 532-550.

Ellinger, A.E., Ketchen, D.J., Hult, G.T.M., Elmadag, A.B. \& Richey, R.G. (2008), "Market orientation, employee development practices, and performance in logistics service provider firms", Industrial Marketing Management, Vol. 37, No. 4, pp. 353-366.

Eltayeb, T.K. \& Zailani, S. (2009), "Going green through green supply chain initiatives towards environmental sustainability", Operations and Supply Chain Management, Vol. 2, No. 2, pp. 93-110.

Evangelista, P. \& Sweeney, E. (2006), "Technology usage in the supply chain: the case of small 3PLs", International Journal of Logistics Management, Vol. 17, No. 1, pp. 55-74.

Flint, D.J., Larsson, E. \& Gammelgaard, B. (2008), "Exploring process for customer value insights, supply chain learning and innovation: an international study", Journal of Business Logistics, Vol. 28, No. 1, pp. 257-281.

Flint, D.J., Larsson, E., Gammelgaard, B. \& Mentzer, J. (2005), "Logistics innovation: A customer value-oriented social process", Journal of Business Logistics, Vol. 26, No. 1, pp. 113-147.

Flowers, A.D., Cort, S.G., Artman, L.B. \& Ballou, R.H. (2008), A Focused Innovation model for Logistics Service Providers, in Wagner \& Busse (eds.) Managing Innovation - The New Competitive Edge for Logistics Service Providers, $1^{\text {st }}$ ed., Haupt Verlag, Berne, Switzerland, pp.79-106.

Franklin, J. R. (2008), Managing the Messy Process of Logistics Service Innovation, in Wagner \& Busse (eds.) Managing Innovation - The New Competitive Edge for Logistics Service Providers, $1^{\text {st }}$ ed., Haupt Verlag, Berne, Switzerland, pp.153-170.

Froehle, C.M. \& Roth, A.V. (2007), "A Resource-Process Framework of New Service Development", Production and Operations Management, Vol. 16, No. 2, pp. 169-188.

Froehle, C.M. \& Roth, A.V. (2004), "New measurement scales for evaluating perceptions of the technology-mediated customer service experience", Journal of Operations Management, Vol. 22, No. 1, pp. 1-21. 
Froehle, C.M., Roth, A.V., Chase, R.B. \& Voss, C.A. (2000), "Antecedents of New Service Development Effectiveness - An Exploratory Examination of Strategic Operations Choices", Journal of Service Research, Vol. 3, No. 1, pp. 3-17.

Fugate, B.S., Mentzer, J.T. \& Flint, D.J. (2008), "The role of logistics in market orientation", Journal of Business Logistics, Vol. 29, No. 2, pp. 1-26.

Gammelgaard, B. (2008), Logistics Innovation Processes: Potential Pitfalls and How to Prevent Them - AN LSP Perspective, in Wagner \& Busse (eds.) Managing Innovation - The New Competitive Edge for Logistics Service Providers, $1^{\text {st }}$ ed., Haupt Verlag, Berne, Switzerland, pp.135-152.

Gammelgaard, B. \& Prockl, G. (2012), "Innovating for Green Supply Chain Management: The logistics service providers perspective" in Carlsson et al. (eds.) Rethinking Transport in the Öresund Region: Policies, Strategies and Behaviours, Chapter 10, pp. 165-182.

Gammelgaard, B., Prockl, G., Thordadottir, K. \& Kinra, A. (2012), "Service innovation and Sustainability in the Danish Logistics Sector", in proceedings of the $24^{\text {th }}$ Annual Nordic Logistics Research Network Conference, University of Turku, Finland, pp. 172-186.

Goldstein, S.M., Johnston, R., Duffy, J. \& Rao, J. (2002), “The service concept: the missing link in service design research?", Journal of Operations Management, Vol. 20, No. 2, pp. 121-134.

Hertz, S. \& Alfredsson, M. (2003), "Strategic development of third party logistics providers", Industrial Marketing Management, Vol. 32, No. 2, pp. 139-149.

Ho, Y-H., Lin, C-Y. \& Chiang, S-H. (2009), "Organisational Determinants of Green Innovation Implementation in the Logistics Industry", The International Journal of Organisational Innovation, Vol. 2, No. 1, pp. 5-12.

Hong, P., Kwon, H.B., \& Roth, J.J. (2009), "Implementation of strategic green orientation in supply chain. An empirical study of manufacturing firms", European Journal of Innovation Management, Vol. 12, No. 4, pp. 512-532.

Hull, F.M., (2004), “A Composite Model of Product Development Effectiveness: Application to Services", IEEE Transactions on Engineering Management, Vol. 51, No. 2, pp. 162-172.

Isaksson, K. (2012), Logistics Service Providers going green - insights from the Swedish market, Department of Management and Engineering, Licentiate Thesis no. 1518, Linköping University, Linköping.

Isaksson, K. \& Huge-Brodin, M. (2013), "Understanding efficiencies behind logistics service providers' green offerings", Management Research Review, Vol. 36, No. 3, pp. 216-238.

Isaksson, K., Huge-Brodin, M., Liimatainen, H. \& Evangelista, P. (2012), "Who is responsible when it finally happens? - functional involvement in adopting green initiatives among logistics service providers" in Wilding, R., (ed.), The $17^{\text {th }}$ Annual LRN Conference winning the Supply Chain triathlon: Creating Social, Economic and Environmental Value, pp. $1-8$. 
Jaw, C., Lo, J-Y. \& Lin, Y-H. (2010), "The determinants of new service development: Service characteristics, market orientation, and actualizing innovation effort", Technovation, Vol. 30, No. 4, pp. 265-277.

Johne, A. \& Davies, R. (2000), "Innovation in medium-sized insurance companies: How marketing adds value”, International Journal of Bank Marketing, Vol. 18, No. 1, pp. 6-14.

Johne, A. \& Storey, C. (1998), "New service development: a review of the literature and annotated bibliography", European Journal of Marketing, Vol. 32, No. 3, pp. 184-251.

Johnson, S.P., Menor, L.J., Roth, A.V. \& Chase, R.B. (2000), "A Critical Evaluation of the New Service Development Process: Integrating Service Innovation and Service Design" in Fitzsimmons, J.A. \& Fitzsimmons, M.J. (eds.), New Service Development - Creating Memorable Experiences, Sage Publications Inc, Thousand Oaks, US, pp. 1-33.

Jumadi, H. \& Zailani, S. (2010) "Integrating Green Innovations in Logistics Services Towards Service Sustainability: A Conceptual Paper”, Environmental Research Journal, Vol. 4, No. 4, pp. 261-271.

Kelly, D. \& Storey, C. (2000), "New service development: initiation strategies", International Journal of Service Industry Management, Vol. 11, No. 1, pp. 45-62.

Kirca, A.H., Jayachandran, S. \& Bearden, W.O. (2005), "Market Orientation: A MetaAnalytic Review and Assessment of Its Antecedents and Impact on Performance", Journal of Marketing, Vol. 69, No. 2, pp. 24-41.

Kuczmarski, T.D. \& Mandolia, R., (2012), "Service Development" in Kahn, K.B. (ed.), The PDMA Handbook of New Product Development, $3^{\text {rd }}$ edition, John Wiley \& Sons, New Jersey, US, pp.51-67.

Kuester, S., Schuhmacher, M.C., Gast, B. \& Worgul, A. (2013), "Sectorial Heterogeneity in New Service Development: An exploratory Study of Service Types and Success Factors", Journal of Product Innovation Management, Vol. 30, No. 3, pp. 533-544.

Li, Y., Li, L., Liu, Y. \& Wang, L. (2005), "Linking management control system with product development and process decisions to cope with environment complexity", International Journal of Production Research, Vol. 42, No. 12, pp. 2577-2591.

Lieb, K.J. \& Lieb, R.C. (2010), "Environmental sustainability in the third-party logistics (3PL) industry", International Journal of Physical Distribution \& Logistics Management, Vol. 40, No. 7, pp. 524-533.

Lin, C-Y. \& Ho, Y-H. (2011), "Determinants of Green Practice Adoption for Logistics Companies in China", Journal of Business Ethics, Vol. 98, No. 1, pp. 67-83.

Lin, C-Y. \& Ho, Y-H. (2008), “An Empirical Study on Logistics Service Providers' Intention to Adopt Green Innovations", Journal of Technology Management \& Innovation, Vol. 3, No. 1, pp. 17-26. 
Lusch, R.F., Vargo, S.L. \& O’Brien, M. (2007), “Competing through service: Insights from service-dominant logic", Journal of Retailing, Vol. 83, No. 1, pp. 5-18.

Maack, C. (2012), Logistics Service Providers' Environmental Management, Department of Management and Engineering, Licentiate Thesis no. 1551, Linköping University, Linköping.

Mangan, J., Lalwani, C. and Gardner, B. (2004), "Combining quantitative and qualitative methodologies in logistics research", International Journal of Physical Distribution \& Logistics Management, Vol. 34, No. 7, pp. 565-578.

Martinsen, U. (2014), Towards greener supply chains - inclusion of environmental activities in relationships between service providers and shippers, Dissertations, No. 1565, Linköping Studies in Science and Technology, Linköping University.

Martinsen, U. \& Huge-Brodin, M. (2010), "Greening the offerings of logistics service providers" in proceedings of the 22th Annual NOFOMA conference Logistics and Supply Chain Management in a Globalised Economy, University of Southern Denmark, pp. 969-984.

Matthing, J., Sandén, B. \& Edvardsson, B. (2004), "New service development: learning from and with customers", International Journal of Service Industry Management, Vol. 15, No. 5, pp. 479-498.

McKinnon, A. (2010), Environmental Sustainability: A new priority for logistics managers, in McKinnon, A., Cullinane, S., Browne, M. \& Whitening, A., (eds.), Green Logistics Improving the environmental sustainability of logistics, Kogan Page, London, pp. 3-30.

Melton, H.L. \& Hartline, M.D. (2010), "Customer and Frontline Employees on New Service Development Performance”, Journal of Service Research, Vol. 13, No. 4, pp. 411-425.

Mena, C., Christopher, M., Johnson, M. \& Jia, F. (2007), "Innovation in Logistics Services" Report produced at the Centre for Logistics and Supply Chain Management at Cranfield School of Management on behalf of National Endowment for Science, Technology and the Arts (NESTA), pp.1-32.

Menor, L.J. \& Roth, A.V. (2008), "New Service Development Competence and Performancfe: An Empirical Investigation in Retail Banking”, Production and Operations Management, Vol. 17, No. 3, pp. 267-284.

Menor, L.J. \& Roth, A.V. (2007), "New service development competence in retail banking: Construct development and measurement validation", Journal of Operations Management, Vol. 25, No. 4, pp. 825-846.

Menor, L.J., Tatikonda, M.V. \& Sampson, S.E. (2002), "New service development: areas for exploitation and exploration", Journal of Operations Management, Vol. 20, No. 2, pp. 135157.

Mentzer, J.T. \& Kahn, K.B. (1995), “A framework of logistics research”, Journal of Business Logistics, Vol. 16, No. 1, pp. 231-250. 
Meredith, J. (1998), "Building operations management theory through case and field research", Journal of Operations Management, Vol. 16, No. 4, pp. 441-454.

Michel, S., Brown, S.W. \& Gallan, A.S. (2008), "An expanded and strategic view of discontinuous innovations: deploying a service-dominant logic", Journal of the Academy of Marketing Science, Vol. 36, No. 1, pp. 54-66.

Nilsson, F. \& Gammelgaard, B. (2012), "Moving beyond the systems approach in SCM and logistics research", International Journal of Physical Distribution \& Logistics Management, Vol. 42, No. 8, pp. 764-783.

Näslund, D. (2002), "Logistics needs qualitative research - especially action research", International Journal of Physical Distribution \& Logistics Management, Vol. 32, No. 5, pp. 321-338.

Oke, A. (2008), Barriers to Innovation Management in Logistics Service Providers, in Wagner \& Busse (eds.) Managing Innovation - The New Competitive Edge for Logistics Service Providers, $1^{\text {st }}$ edition, Haupt Verlag, Berne, Switzerland, pp.13-30.

Ordanini, A. \& Maglio, P.P. (2009), "Market Orientation, Internal Process, and External Network: A Qualitative Comparative Analysis of Key Decisional Alternatives in the New Service Development", Decision Sciences, Vol. 40, No. 3, pp. 601-625.

Ottenbacher, M.C. \& Harrington, R.J. (2010) "Strategies for achieving success for innovative versus incremental new services”, Journal of Services Marketing, Vol. 24, No. 1, pp. 3-15.

Page, A.L. \& Schirr, G.R. (2008), "Growth and Development of a Body of Knowledge: 16 Years of New Product Development Research, 1989-2004", Journal of Product Innovation Management, Vol. 25, No. 3, pp. 233-248.

Panayides, P.M. (2007), "Effects of organisational learning in Third-Party Logistics", Journal of Business Logistics, Vol. 28, No. 2, pp. 133-158.

Panayides, P.M. \& So, M. (2005), "Logistics service provider - client relationships", Transportation Research Part E, Vol. 41, No. 3, pp. 179-200.

Papastathopoulou, P. \& Hultink, E. J. (2012), "New Service Development: An Analysis of 27 Years of Research", Journal of Product Innovation Management, Vol. 29, No. 5., pp. 705714.

Pekkarinen, S. \& Ulkuniemi, P. (2008), "Modularity in developing business services by platform approach", The International Journal of Logistics Management, Vol. 19, No. 1, pp. 84-103.

Pieters, R., Glöckner, H-H., Omta, O. \& Weijers, S. (2012), "Dutch Logistics Service Providers and Sustainable Physical Distribution: Searching for Foucs", International Food and Agribusiness Management Review, Vol. 15, No. B, pp. 107-126.

PostNord (2014), PostNord Nyhetsposten, Internal magazine of PostNord, No. 2. 
PostNord (2012), Nordisk Logistikbarometer, [Online], Available:

http://www.postnord.com/sv/Media/Publikationer/Logistik/, Accessed on the 17 $7^{\text {th }}$ of August, 2013.

PostNord (2011, 2012), Annual Report with Sustainability Report 2011, 2012, [Online], Available: http://www.postnord.com/en/Investor-Relations/Finansiell-information/2013, Accessed on the $11^{\text {th }}$ of September, 2013.

Prockl, G. \& Gammelgaard, B.(2012), Sustainability and the Logistics Service Industry - Red thread from a Workshop, [Online], Available: http://openarchive.cbs.dk/handle/10398/8585, Accessed on the $7^{\text {th }}$ of October, 2013.

Prockl, G., Pflaum, A. \& Kotzab, H. (2012), "3PL factories or lernstatts? Value-creation models for 3PL service providers", International Journal of Physical Distribution \& Logistics Management, Vol. 42, No. 6, pp. 544-561.

Rajesh, R., Pugazhendhi, S., Ganesch, K., Muralidharan, C. \& Sathiamoorthy, R. (2011), "Influence of 3PL service offerings on client performance in India", Transportation Research Part E, Vol. 47, No. 2, pp. 149-165.

Rossi, S., Colicchia, C., Cozzolino, A. \& Christopher, M. (2013), "The logistics service providers in eco-efficiency innovation: an empirical study", Supply Chain Management: An International Journal, Vol. 18, No. 6, pp. 583-603.

Schleimer, S.C. \& Schulman, A.D. (2011), "When intra-firm and inter-firm collaborations cooccur: comparing their impact across new services versus new products innovations", International Journal of Innovation Management, Vol. 15, No. 5, pp. 869-898.

Scheuing, E.Z. \& Johnson, E.M. (1989), "A proposed model for new service development", Journal of Services Marketing, Vol. 3, No. 2, pp. 25-34.

Shen, H., Wang, L., Xu, Q., Li, Y. \& Liu, X. (2009), "Toward a Framework of Innovation Management in Logistics Firms: A Systems Perspective”, Systems Research and Behavioural Science, Vol. 26, No. 2, pp. 297-309.

Shostack, L.G. (1987), "Service Positioning Through Structural Change", Journal of Marketing, Vol. 51, No. 1, pp. 34-43.

Siggelkow, N. (2007), "Persuasion with case studies", Academy of Management Journal, Vol. 50, No. 1, pp. 20-24.

Soinio, J., Tanskanen, K. \& Finne, M. (2012), "How logistics-service providers can develop value-added services for SMEs: a dyadic perspective", The International Journal of Logistics Management, Vol. 23, No. 1, pp. 31-49.

Speh, T.W. (2008), Innovative LSP Services: The Marketing Challenges, in Wagner \& Busse (eds.) Managing Innovation - The New Competitive Edge for Logistics Service Providers, $1^{\text {st }}$ ed., Haupt Verlag, Berne, Switzerland, pp.31-60. 
Stentoft, A. \& Halldorsson, A. (2002), "Logistics knowledge creation: reflections on content, context and processes", International Journal of Physical Distribution \& Logistics Management, Vol. 32, No. 1, pp. 22-40.

Stock, J.R. (1997), "Applying theories from other disciplines to logistics", International Journal of Physical Distribution \& Logistics, Vol. 27, No. 9/10, pp. 515-539.

Storey, C. \& Easingwood, C.J. (1999), "Types of New Product Performance: Evidence from the Consumer Financial Service Sector", Journal of Business Research, Vol. 46, No. 2, pp. 193-203.

Storey, C. \& Hull, F.M. (2010), "Service development success: a contingent approach by knowledge strategy", Journal of Service Management, Vol. 21, No. 2, pp. 140-161.

Stuart, I., McCutcheon, D., Handfield, R., McLachlin, R. \& Samson, D. (2002), "Effective case research in operations management: a process perspective", Journal of Operations Management, Vol. 20, No. 5, pp. 419-433.

Sundbo, J. (1997), "Management of Innovation in Service", Service Industries Journal, Vol. 17, No. 3, pp.432-455.

Syson, F. \& Perks, H. (2004), "New service development: a network perspective", Journal of Services Marketing, Vol. 18, No. 4, pp. 255-256.

Sweeney, E. \& Evangelista, P. (2005), “3PL definition and taxonomy”, Logistics Solutions, Vol. 7, No. 2, pp. 9-10.

Third-Party Logistics Study, (2013), The 17 th Annual Third-Party Logistics Study, [Online], Available: http://www.panalpina.com, Accessed on the 20 ${ }^{\text {th }}$ of September, 2013.

Third-Party Logistics Study, (2012), The $16^{\text {th }}$ Annual Third-Party Logistics Study, [Online], Available: http://www panalpina.com, Accessed on the 17 $7^{\text {th }}$ of August, 2012.

Treacy, M. \& Wiersema, F. (1995), The Discipline of Market Leaders - choose your customers, narrow your focus, dominate your market, Addison-Wesley Publishing Company, New York, US.

van der Veeken, D.J.M. \& Rutten, W.G.M.M. (1998), "Logistics Service Management: Opportunities for Differentiation" The International Journal of Logistics Management, Vol. 9, No. 2, pp. 91-98.

Vargo, S.L. \& Lusch, R.F. (2004), "Evolving to a New Dominant Logic for Marketing", Journal of Marketing, Vol. 68, No. 1, pp. 1-17.

Veflen Olsen, N. \& Sallis, J. (2006), "Market scanning for new service development", European Journal of Marketing, Vol. 40, No. 5, pp. 466-484.

Vermeulen, P.A.M. \& Dankbaar, B. (2002), "The organisation of product innovation in the financial sector”, The Service Industries Journal, Vol. 22, No. 3, pp. 77-98. 
Voss, C., Tsikriktsis, N. \& Frohlich, M. (2002), "Case research in operations management", International Journal of Operations \& Production Management, Vol. 22, No. 2, pp. 195-219.

Wagner, S.M. (2013), "Partners for Business-to-Business Service Innovation", IEEE Transactions of Engineering Management, Vol. 60, No.1, pp. 113-123.

Wagner, S. M. (2008), "Innovation management in the German transportation industry", Journal of Business Logistics, Vol. 29, No. 2, pp. 215-231.

Wagner, M. \& Busse, C. (2008), Managing Innovation at Logistics Service Providers - An Introduction, in Wagner \& Busse (eds.) Managing Innovation - The New Competitive Edge for Logistics Service Providers, $1^{\text {st }}$ ed., Haupt Verlag, Berne, Switzerland, pp. 1-12.

Wagner, M. \& Franklin, J. (2008), “Why LSPs don't leverage innovations”, CSCMP's Supply Chain Quarterly, Vol. 4, No. 2, pp. 67-71.

Wagner, S.M. \& Sutter, R. (2012), "A qualitative investigation of innovation between thirdparty logistics providers and customers", International Journal of Production Economics, Vol. 140, No. 2, pp. 944-958.

Wallenburg, C.M. (2009), "Innovation in Logistics outsourcing relationships: proactive improvements by Logistics service providers as a driver of customer loyalty", Journal of Supply Chain Management, Vol. 45, No. 2, pp. 75-93.

Wolf, C. \& Seuring, S. (2010), "Environmental impacts as buying criteria for third party logistical services", International Journal of Physical Distribution \& Logistics Management, Vol. 40, No. 1/2, pp. 84-102.

Yazdanparast, A., Manuj, I. \& Swartz, M. (2010), "Co-creating logistics value: a servicedominant logic perspective", The International Journal of Logistics Management, Vol. 21, No. 3, pp. 375-403.

Yin, R.K. (2009), Case Study research - Design and Methods, $4^{\text {th }}$ ed., SAGE Publications Inc, Thousands Oaks, US.

Zailani, S., Amran, A. \& Jumadi, H. (2011), "Green Innovation Adoption among Logistics Service Providers in Malaysia: An Exploratory Study on the Managers' Perceptions", International Business Management, Vol. 5, No. 3, pp. 104-112.

Zhou, G.H. \& Wang, X.Q. (2012), "The research on the process of new service development in logistics enterprises", International Journal of Networking and Virtual Organisations, Vol. 10, No. $3 / 4$, pp. 334-345. 


\section{APPENDIX}

\section{THE LICENTIATE THESIS}

Isaksson, K. (2012), Logistics service providers going green - insights from the Swedish market, Thesis No. 1518, Linköping Studies in Management and Economics, Linköping University. 

Linköping Studies in Science and

Technology, Thesis No. 1518

LiU-TEK-LIC 2012:03

\title{
Logistics Service Providers going green - insights from the Swedish market
}

\author{
Karin Isaksson
}

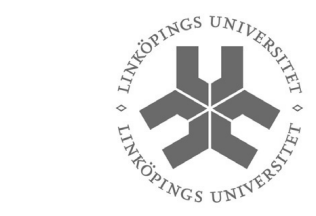

Linköpings universitet

2012

Department of Management and Engineering

Linköping University, SE-581 83 Linköping 
(C) Karin Isaksson, 2012

Linköping studies in science and technology, Thesis No. 1518

LiU-TEK-LIC 2012:03

ISBN: 978-91-7519-970-2

ISSN: 0280-7971

Printed by: LiU-Tryck, Linköping

Distributed by:

Linköping University

Department of Management and Engineering SE-581 83 Linköping, Sweden

Tel: +46 13 281000, fax: +4613281873 


\section{ABSTRACT}

During recent years pressures on the logistics and transport industry to involve and engage more in environmental work have increased. Governmental awareness of environmental impact has led to an increased pressure on the logistics and transport industry to reduce the emissions incurred by their operations. In addition, customers have become more interested and demanding regarding green initiatives in their purchasing of transport services. Although logistics service providers (LSPs) are becoming more aware of environmental problems, the development of green initiatives in the logistics and transport industry is described in the literature as being in its infancy phase. Considering the scarcity of studies on LSPs' green initiatives, a study that reveals potential factors influencing the adoption of green initiatives may be helpful to fill the knowledge gap and provide opportunities for further research in this field.

The purpose of this licentiate thesis is therefore to describe how different factors can affect the adoption of green initiatives among LSPs, and how the adoption of green initiatives can be reflected in the service offering. This includes identifying different kinds of triggers, drivers and barriers, as well as firm characteristics and describing how these factors can affect the adoption of green initiatives. Furthermore, ways in which the adoption of green initiatives can be reflected in the service offering are exemplified in order to answer the second part of the purpose.

The theoretical base in this licentiate thesis can be linked to general environmental logistics literature, sustainable service, and product development research. The research in this study is in its nature explorative and empirical data have been obtained from a cross case study of six companies, and a survey study investigating LSPs active on the Swedish market.

Based on the findings from the analysis, the LSPs studied have commenced to adopt green initiatives since they experience the pressure to adapt to future expectations and, understand the consequences. This will enable the LSPs to respond to the increasing and more global competition. Although increased competiveness seems to act as a trigger, the role of competitors as a driver affecting the adoption of green initiatives among LSPs are neither stressed in the case study nor singled out in the survey study as a significant driver. Increased, interest from customers and decisions from top management are both highlighted as triggers for LSPs to start adopting green initiatives, in the literature as well as among the LSPs studied.

The role of customers and top management also seem to be prominent drivers in the further green development. By adopting green initiatives, LSPs strive to win new customers and there is also a desire to improve customer relationships. Furthermore, the engagement and support from top management can be crucial for how successfully the adoption of green initiatives is integrated into the company and received by the employees. In addition, among the LSPs studied, their employees can be considered as an essential driver and a resource when adopting green initiatives. 
Regarding the studied barriers, financial and economical barriers emerge as crucial when LSPs adopt green initiatives. In addition, customers are also perceived as a barrier among the LSPs studied due to reasons such as lack of customer support, unwillingness to pay for green initiatives as well as unclear and unreasonable green requirements from customers.

Another finding from the study is that size of the company, type of service offerings provided, as well as type of customers seems to play an essential role when LSPs adopt green initiatives. The analysis indicates that firm characteristics can moderate the effects of different drivers and barriers and suggests that firm characteristics rather effect the adoption of green initiatives indirectly than directly.

Finally, despite the early phase of LSPs greening process, the analysis presents three different patterns that reflect the current behaviour and anticipations among the LSPs studied. The development of green service offerings includes the internal work as well as the explicit service offering. This in turn indicates that the selected green approach and how LSPs choose to react and adopt green initiatives will in the end be either directly or indirectly reflected in the service offering. 


\section{ACKNOWLEDGEMENTS}

The first part of my journey to become a $\mathrm{PhD}$ has come to its end. Ever since the beginning, it has been an adventure with a lot of wisdom, beautiful memories and of course a lot of hard work. During my journey I have gotten the chance to meet and get to know a lot of amazing and talented people. Some of them have just crossed my road but for those who have been standing by my side from the beginning or during most part of my journey I would like to dedicate a special THANK YOU.

Firstly, my deepest gratitude is to my supervisors Maria Huge-Brodin and Maria Björklund for all their encouragements, thoughtful comments and support throughout this research. Also, I am very grateful for the guidance and the inspiration you have been given me.

I also want to thank Erik Sandberg for his effort to read and give valuable feedback and comments on the draft of this thesis. Mats Abrahamsson, thank you for taking the time to review articles and give valuable advices when needed. Also thanks to Kristina Dalberg for helping me with the layout in the end of this process.

Another thank you goes to VINNOVA for funding the research project, which I have been a part of. Furthermore, I would like to thank all respondents of the case companies studied and to all respondents who answered the questionnaire.

And a lot of thank you to the "logistics girls", Christina, Malin and Uni, not only for being great colleagues but also for being good friends of mine. Thank you for all laughs and happy moments. And to the rest of my wonderful colleagues at the Logistics Division, thank you for always brighten my days at work.

Last but definitely not least, a special and enormous huge thank you to my dear family and all my fantastic friends - I am very lucky to have you all in my life. My last special thank you goes to Stephan, whose encouragement and eyes opened comments have been very valuable and helped me throughout this process.

Once again thank you all!

While I am writing these very last words, I am at the same place where I once took the decision to start this journey. I truly hope that all of you want to be by my side on my continued journey to become a PhD.

Mossänder, January 2012

Karin Helena Isaksson 



\section{Table of Content}

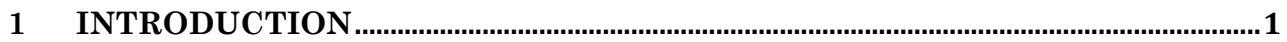

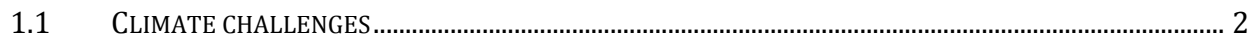

1.2 THE DEVELOPMENT WITHIN THE LSP INDUSTRY …………................................................................ 3

1.3 NEED FOR GREEN RESEARCH WITHIN THE LSP INDUSTRY ................................................................... 5

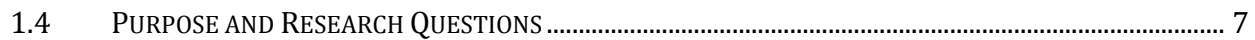

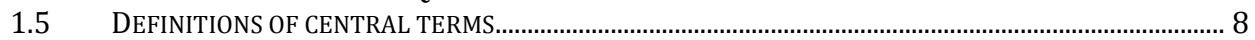

1.6 SCOPE AND FOCUS OF THE LICENTIATE THESIS …............................................................................. 9

1.7 THE OUTLINE OF THE LICENTIATE THESIS.....................................................................................

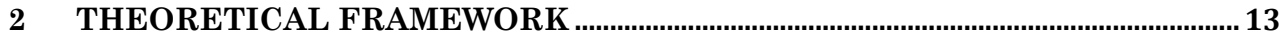

$2.1 \quad$ LOGISTICS OFFERINGS IN GENERAL

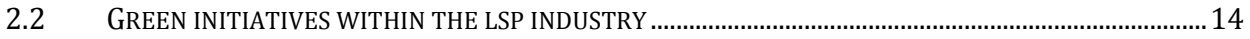

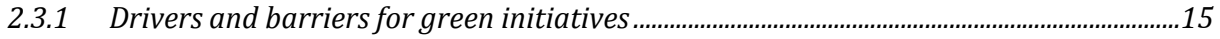

2.3.2 Firm characteristics influencing on green initiatives...........................................................18

$3 \quad$ RESEARCH APPROACH.................................................................................................19

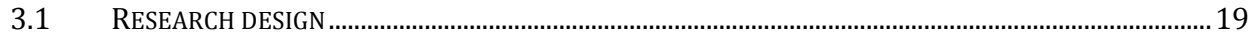

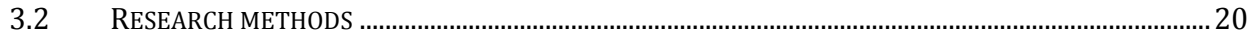

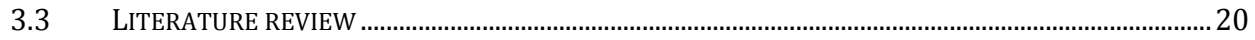

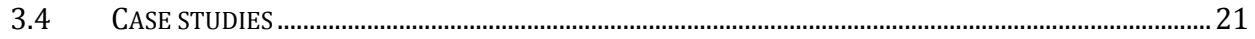

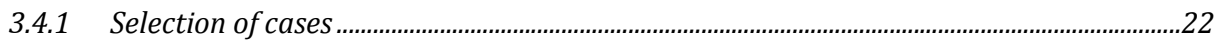

3.4.2 Descriptions of cases.........................................................................................................................

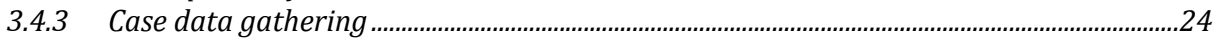

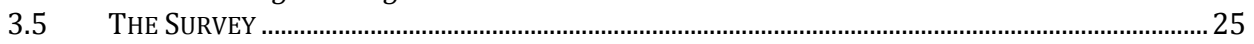

3.5.1 Preparation of the questionnaire survey ....................................................................................25

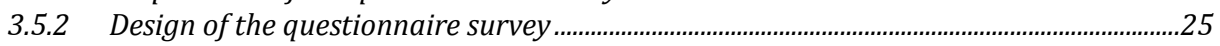

3.5.3 Sample profile and data collection...................................................................................................26

3.6 ANALYSIS UNIT AND ANALYSIS OF THE RESEARCH QUESTIONS.........................................................2

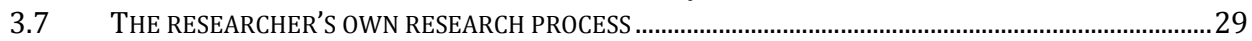

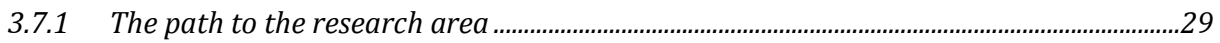

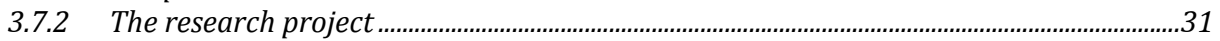

3.7.3 The researcher's contribution to the Papers ..........................................................................31

4 SUMMARY OF THE APPENDED PAPERS ……....................................................... 33

4.1 PAPER 1 DEVELOPING SUSTAINABLE LOGISTICS SERVICES ................................................................33

4.2 PAPER 2 GREEN INITIATIVES IN THE TRANSPORT AND LOGISTICS SERVICE INDUSTRY: AN

EXPLORATORY CASE STUDY OF LOGISTICS SERVICE PROVIDERS...................................................................35

4.3 PaPer 3 Understanding EFficiencies Behind Logistics Service Providers' GreEn

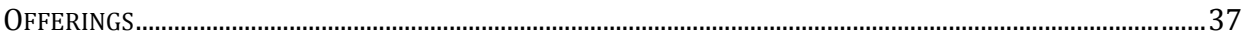

4.4 PAPER 4 THE CHALLENGE AND ADOPTION OF GREEN INITIATIVES FOR TRANSPORT AND LOGISTICS

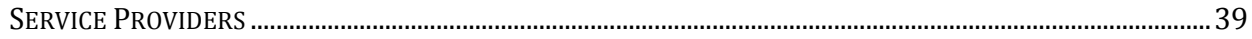

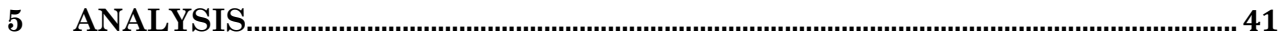

5.1 HOW CAN DIFFERENT TRIGGERS INITIATE THE ADOPTION OF GREEN INITIATIVES? .......................... 41

5.2 HOW CAN DIFFERENT DRIVERS AND BARRIERS AFFECT THE ADOPTION OF GREEN INITIATIVES?....45

5.2.1 How can different drivers affect the adoption of green initiatives? .....................................46

5.2.2 How can different barriers affect the adoption of green initiatives?...................................51

5.3 HOW CAN FIRM CHARACTERISTIC AFFECT THE ADOPTION OF GREEN INITIATIVES? ...........................56

5.4 IN WHICH WAYS CAN THE ADOPTION OF GREEN INITIATIVES BE REFLECTED IN THE SERVICE

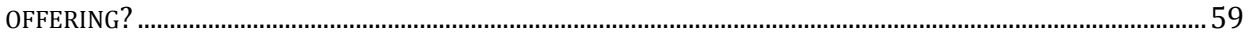

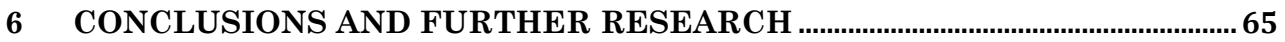




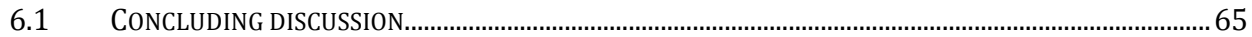

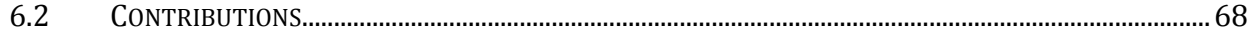

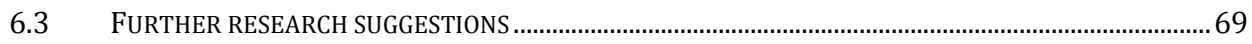

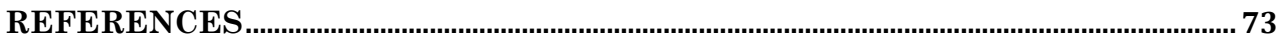

APPENDIX 1: $\quad$ INTERVIEW GUIDE

APPENDIX 2: $\quad$ CASE REPORT

APPENDIX 3: $\quad$ THE QUESTIONNAIRE

APPENDIX 4: $\quad$ PAPER 1: DEVELOPING SUSTAINABLE LOGISTICS SERVICES

APPENDIX 5: $\quad$ PAPER 2: GREEN INITIATIVES IN THE TRANSPORT AND LOGISTICS SERVICE INDUSTRY: AN EXPLORATORY CASE STUDY OF LOGISTICS SERVICE PROVIDERS

APPENDIX 6: $\quad$ PAPER 3: UNDERSTANDING THE EFFICIENCIES BEHIND LOGISTIC SERVICE PROVIDERS' GREEN OFFERINGS

APPENDIX 7: $\quad$ PAPER 4: THE CHALLENGE AND ADOPTION OF GREEN INITIATIVES FOR TRANSPORT AND LOGISTICS SERVICE PROVIDERS 


\section{List of Figures}

Figure 1 Emissions ( $\mathrm{CO}_{2}$ eq.) by different sectors in Sweden (modified and adapted from WWF, 2009b)

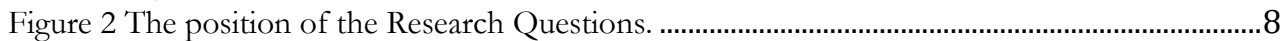

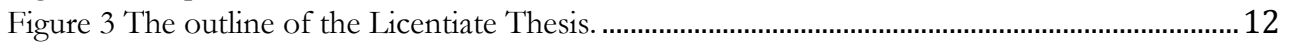

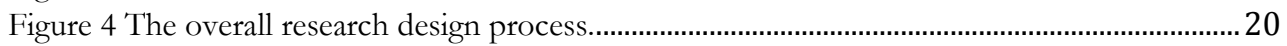

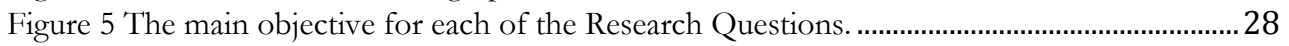

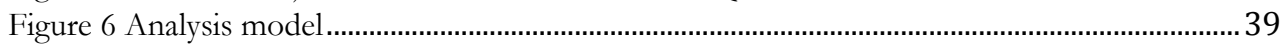

Figure 7 Regression analysis of strong drivers and green initiatives................................................. 40

Figure 8 The position of Research Question 1................................................................................. 41

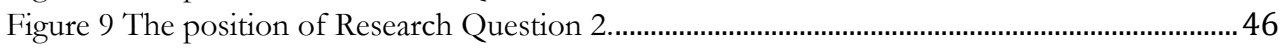

Figure 10 The position of Research Question 3. .............................................................................. 57

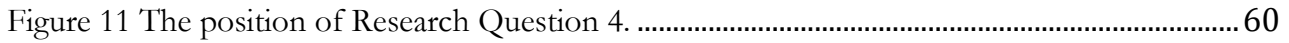

\section{List of Tables}

Table 1 Research methods applied for the appended Papers............................................................ 20

Table 2 The link between the research questions and the appended Papers.................................... 28

Table 3 Framework of environmentally sustainable services for logistics companies.....................34

Table 4 Summary of the most evident case characteristics identified according to the 6 different

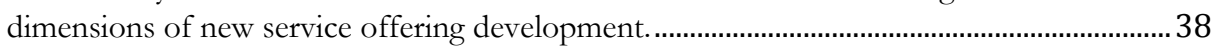

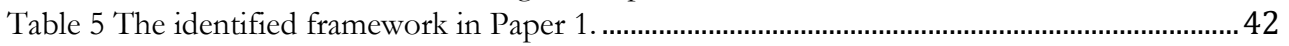

Table 6 The impact of different stakeholders among the studied case companies..........................47

Table 7 Stakeholders' impact on the adoption of green initiatives...................................................49

Table 8 The impact of the studied reasons acting as drivers.........................................................50

Table 9 Reasons studied that drive the adoption of green initiatives...............................................51

Table 10 The impact of internal barriers among the studied case companies..................................52

Table 11 Internal barriers to the adoption of green initiatives.........................................................54

Table 12 The impact of external barriers among the studied case companies................................54

Table 13 External barriers affecting the adoption of green initiatives. ............................................56

Table 14 Parts of the framework presented in Paper 1......................................................................... 60 



\section{INTRODUCTION}

In order to give the reader an insight into the main contents of this licentiate thesis, this chapter addresses how the logistics service providers' industry has developed during recent years as well as how this industry has been affected by the ongoing debate in society. The background leads then into the purpose and the research questions of this thesis. In addition, the scope and the focus of the study as well as definitions of central concept and terms are presented, and the chapter ends with a presentation of the outline of this licentiate thesis.

There are always changes in all industries affected by different dominant drivers. In recent years, it has become common to have an increasing focus on logistics, as companies have seen new opportunities to streamline their operations and increase their competitiveness (Zacharia and Mentzer, 2007). Some examples of drivers that have been identified for business change and new structures in the logistics chains during the last decade are presented below.

Let us start with the globalisation of supply chains, which has prompted many firms to develop logistics as part of their corporate strategy (McGinnis and Kohn, 2002). One effect of this is the fact that logistics has been given a more prominent and strategic role within many companies (Zacharia and Mentzer, 2007). These authors also stress that logistics has become a source of competitive advantage, especially through transportation deregulation and improvements in information technology which have enabled companies to gain competitive advantage through competence in delivery speed, reliability, responsiveness and low cost distribution.

Due to the introduction of new information and communication technology (ICT), new opportunities have been created in controlling and follow-up logistics development, such as increased the possibility of transferring information both geographically and between different supply chain participants (Lemoine \& Skjøtt-Larsen, 2004).

Another prominent driver that has affected the reconfiguration of the supply chains is market requirements. Higher requirements on customized products and services have led to new logistics solutions, where integration and cooperation is increasingly important when the dependence on external actors' performance directly affects the business delivery service. In other words, this change is driven by new demands from customers, competitors, new solutions, more advanced concepts and new roles and collaborations. Companies face new challenges to change and organize to be able to offer competitive solutions in the new emerging logistics network. Changes in the logistics chains will also produce changes in the demands for freight transport (Drewes Nielsen et al., 2003). In order to meet these demands and to deliver products and services quickly to customers, many companies seek to outsource their logistics activities to logistics service providers (LSPs). This reflects the trend of using LSPs in order to satisfy the increasing need for logistics services (Lieb and Miller, 2002). Even though LSPs have an important role in logistics, Fabbe-Costes et al. (2009) indicate that LSPs often are seen as the neglected actors of supply chain integrations. However, these players have lately received increased 
attention, due to the negative impact on the natural environment caused by transport and logistics operations. The following section will present some of the set targets and challenges connected to climate change that have been directed towards the logistics and transport industry.

\subsection{Climate CHALLENGES}

As the general awareness of the changing global environment is increasing, governments are starting to react more strongly and more actively to counteract the negative impact on the environment caused by society and businesses. Reductions of greenhouse emissions are one common objective that has been set in order to decrease the effects of global warming. These set targets are related to the UN's convention on climate change and the associated Kyoto Protocol. A joint study between Allianz and WWF (2009a) provides an analysis of the G8's national efforts to tackle climate change. However, different nations have reacted differently to climate challenge for reasons such as level of development, industrial structure, availability of natural resources, public perceptions and economic activities that result in greenhouse gas (GHG) emissions. For example, Germany has set a national emission target that envisages a $40 \%$ emissions reductions by 2020 compared to 1990 levels, but show no convincing strategy for low carbon transition in the transport sector. Another example, United Kingdom, has set a target to reduce GHG emissions by at least $34 \%$ by 2020 and at least $80 \%$ by 2050 compared to 1990 levels. (WWF, 2009a) Hence, in order to reach these targets, new policies and measures focusing on the transport sector are needed, especially increased improvements within the road freight transport segment (Piecyk, 2010a).

After receiving a feeling how other countries have responded, the question remains; how has Sweden reacted and tackled the climate change challenges? The Swedish national targets are overall more challenging than the EU targets, and Sweden has the ambition to reach a $40 \%$ reduction in GHG emissions outside the European Emissions Trading System by 2020 (2/3 domestic, 1/3 offsets) and aim for a zero net GHG emissions by 2050 (compared to 1990 levels). (WWF, 2009b). The impact of emissions ( $\mathrm{CO}_{2}$ eq.) from different sectors in Sweden is illustrated in Figure 1 below.

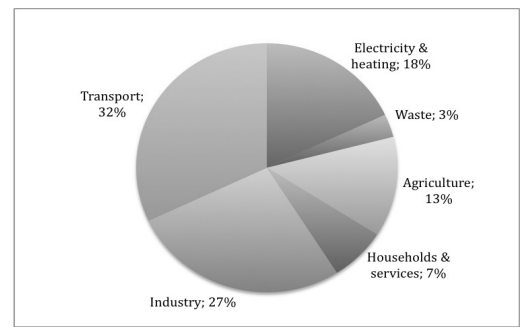

Figure 1 Emissions ( $\mathrm{CO}_{2}$ eq.) by different sectors in Sweden (modified and adapted from WWF, 2009b).

As Figure 1 shows, transport sector represents about 32\% of the total emissions $\left(\mathrm{CO}_{2}\right.$ eq.) and thus has a greater impact than the other sectors. In addition, the transport sector is also the one with the fastest growth (Trafikverket, 2010). The transport sector's carbon footprint is dominated by road transports (including both passenger and freight transports), which constitute about $95 \%$ of the transport sector's domestic $\mathrm{CO}_{2}$ 
emissions. (Transportstyrelsen, 2011). However, during the 2000s, the increase in emissions from road transports has been dampened by the increased use of alternative fuels and more fuel-efficient vehicles (Trafikverket, 2010). Nevertheless, this has not been enough to compensate for the increased traffic growth, and as a result, the emissions increased by about 10\% between the years 1990 and 2009 (Transportstyrelsen, 2011). Today the Swedish Transport Agency has a focused environmental approach regarding vehicle and fuel development towards more energy efficiency and an increased proportion of sustainable bio fuels within the road transports sector (ibid.). For example, a set target within this sector is to have a vehicle fleet independent of fossil fuels by 2030 (WWF, 2009b).

However, the overall development within the transport sector continues to progress too slowly to reach the set targets; this applies first and foremost to carbon dioxide emissions (Transportstyrelsen, 2011). This indicates that if the transport sector is going to contribute to reaching the set targets, measures and instruments with a stronger impact are needed. Technical solutions, such as more efficient vehicles as well as an increased proportion of renewable energy and the electrification of road transport will not be enough (ibid.). Therefore, unless there is a change in our behaviour, both in society and among companies, it will lead to increased levels of emissions from transports in the future.

From a company perspective, managers must start questioning themselves how they can green their companies and supply chains in order to contribute to lowering carbon emissions. However, this will not be without challenges. One example of a company that has shown willingness to take on these challenges and considers itself to be proactive in its approach to climate change is Deutsche Post, DHL (DHL, 2010). The CEO of Deutsche Post DHL, Frank Apple states:

"I am sure that the pursuit of sustainability will transform the logistics industry. At the same time, the logistics industry, with its unique position all along the supply chain and its expertise, can offer important assistance to many sectors as they progress towards a low-carbon economy. Logistics can belp foster sustainability.” (p. 10)

In order to better understand how these challenges can be addressed within the LSP industry, the next step is to gain insights into how this industry has developed over the past decades.

\subsection{THE DEVELOPMENT WITHIN THE LSP INDUSTRY}

The logistics and transport business is an industry which over the years, has undergone fundamental changes and where the individual players have faced new challenges of strategic as well as structural nature (Persson and Virum, 2001). For example, the increasing trend towards the outsourcing of the logistics activities has given prominence to the concept of LSPs and seems most likely to continue to grow over the next few years as well (Lieb and Bentz, 2005).

From a traditional point of view, transport is often viewed as separate from the supply chain and is described as the least integrated link, where there is a great focus on cost 
reductions (Stank and Goldsby, 2000). The LSPs have played a supportive role for the supply chain by providing resources, knowledge, utilities or assets for primary members in the supply chain (Spens and Bask, 2002). This view of LSP has started to change, as these actors have become increasingly influential in the context of supply chain, since the extent to which the logistics functions are prudently undertaken will influence the efficiency and consequent performance of the supply chain (Panayides and So, 2005). Moreover, Lieb and Bentz (2004) stress that the services offered by LSPs can clearly be used as an important element of a company's supply chain management strategy, both domestically and internationally. In other words LSPs can play an important role in linking users to their major vendors and customers, and thereby facilitating supply chain integration.

Another change mentioned by Carbone and Stone (2005), is that more consolidation within the 3PL industry is expected. A few market leaders offer a wide range and scope of services, while most other firms have a diversified portfolio of interests. One common conclusion of several studies suggests that the market for logistics and transport services is becoming larger and more complex (see for example Andersson and Norrman, 2002; Hertz and Alfredsson, 2003 and Lieb and Bentz, 2005). This situation which is evolving in the form of growth and diversification, presents opportunities and challenges for companies, which in turn generate new players from different fields that are entering the market and are competing with the traditional transport and warehousing firms (Hertz and Alfredsson, 2003). Example of such new players can be post offices, ICT consultants and info-providers (Carbone and Stone, 2005).

The increased competitions on the market has put a higher pressure on LSPs and Panayides and So (2005) mean that the competitiveness of LSPs will depend to a large extent on their ability to add value to the bottom line of their clients. However, Lemonie and Skjoett-Larsen, (2004) raise the question of how the users of logistics services will satisfy their clients' demand when LSPs are the least integrated link in the supply chain. Therefore, the evolving role of LSPs in the supply chain will change and probably affect their relationships with customers. This in turn will place greater pressure on LSPs to fulfil the new demands. In addition, Hertz and Alfredsson (2003) stress that in order to add customer value, it will be necessary to develop skills, competencies and gain scale/scope advantages that are superior to the competitors'. Other benefits of developing logistics service capabilities mentioned in the literature other than added value to customers, are an increase in market share, customer satisfaction, differentiation-based competitive advantage and facilitation of market segmentation.

The review above, gives a brief description of the changes in market conditions and challenges within the logistics service industry in recent years. Another challenge that the LSP industry faces today, as mentioned earlier in this section, concerns the challenge to create sustainable practice and performance. This is an area that can be seen to be of increasing importance for LSPs as their core activities (e.g. transport activities) have a strong environmental impact. 


\subsection{NEED FOR GREEN RESEARCH WITHIN THE LSP INDUSTRY}

The development of the role of LSPs, as outlined above, puts these actors in a critical position to support efforts aimed at improving the environmental sustainability of supply chain operations.

In the literature, environmental practises in service sectors have started to attract the attention of researchers (Kassinis and Soteriou, 2003). However, most of the literature and empirical studies regarding environmental sustainable improvements have primarily been directed towards manufacturing companies (see, for example Eltayeb and Zailani, 2009; Hong et al., 2009). In relation to the LSPs sector specifically, two recently published literature reviews on LSPs barely mention sustainability related issues (Maloni and Carter, 2006; Selviaridis and Spring, 2007). A literature review conducted by Lin and Ho (2008), revealed that only a limited number of articles have focused on environmental issues in the context of the logistics industry in the last decade. In addition, Lieb and Lieb (2010) also point out that the LSP industry has been given very little attention in green supply chain research. This in turn shows the relevance of exploring the green development among LSPs and according to Lin and Ho (2008):

"Much remains to be learned empirically about the adoption of environmental practices for logistics service providers" (p. 18).

Since the importance of green management has increased over the past decade (e.g. Skjoett-Larsen, 2000 and McKinnon, 2010), this has led into higher demands on companies to deliver products and services to customers in more environmentally friendly ways. Within in the green logistics area, research has been directed towards a variety of areas, for example assessing the environmental impacts of freight transports. Focus has been on different kinds of externalities associated with freight transport (such as effects of atmospheric pollution, noise pollution and accidents), measuring the environmental impact of freight transport as well as different kinds of environmental standards (both mandatory as well as voluntary/management standards). The assessment of environmental impacts at the company and product level has recently attracted an increased focus on GHG emissions (Cullinane and Edwards, 2010). Therefore, concepts such as "carbon foot-printing" have been introduced in order to help companies to understand and manage their GHG emissions. This area has evolved rapidly in recent years, and carbon auditing in the road freight sector has been shown to provide quicker and more cost-effective means of finding opportunities for decarbonisation within a logistics operation (Piecyk, 2010b).

Another area within green logistics concerns the environmental impacts of different kinds of freight transport modes. Advances in vehicle technology and stricter regulations on emission levels have reduced the transport externalities (McKinnon, 2010). However, Woodburn and Whiteing (2010) claim that in order to meet the $\mathrm{CO}_{2}$ emissions target, a more concerted action of transferring freight to "greener" transport modes (for example by rail and water) may be necessary. The green logistics research area, as mentioned above, seems to address a variety of environmental effects and its impact on logistics, whereas the role of LSPs in this context seems to be neglected. 
Pressures on the LSP industry to involve and engage in more environmental work can come from a variety of sources. For example, as mentioned earlier in this section, governmental awareness of environmental impact has led to an increased pressure on the transport industry to reduce the emissions incurred by their operations, and Rothengatter (2010) state that this will have clear consequences for the transport sector. In addition, a recent report conducted by DHL (2010) indicates that customers will become more demanding about green initiatives in their purchasing of transport services. The LSP industry thus faces a huge challenge to green their operations in order to meet the increased demands from governments as well as customers. Hence, before suggesting how LSPs should manage to meet and fulfil these set targets and demands, it is necessary to find out how LSPs respond to different pressures today. As mentioned earlier, different kind of sources can drive a company to green their operations, which makes it interesting to find out what actually "triggers" LSPs to start considering to adopt green initiatives within their companies. Increased pressures from governments as well as green demands from customers are both mentioned above as examples of sources of influence but what role and what influence do these stakeholders have on LSPs regarding the actual adoption of green initiatives?

Even if LSPs are becoming more aware of environmental problems and are working with green initiatives (e.g. DHL, 2010, DB Schenker, 2011), the development of green initiatives in the logistics industry is described in the literature as being in its infancy phase (Lin and Ho, 2008). In order to get a better understanding and clarify what kind of factors affect the greening process among LSPs, it can be fruitful to investigate and consider factors that stimulate (drivers) and inhibit (barriers) LSP to adopt green initiatives. However, Lin and Ho (2008) indicated in their research that all organisations are not exposed to the same type of pressure or to the same extent, which in turn, indicates that different types of LSP may be influenced and affected differently when it comes to greening their businesses, and also that they choose different paths in their work.

Furthermore, the adoption of green initiatives can also be seen as a business opportunity for LSPs and a means to attract new customers (Lieb and Lieb, 2010). However, in order to make this happen, LSPs must consider how their efforts on environmental issues can be addressed towards their customers to obtain their awareness and interest.

Considering the scarcity of studies on LSPs' green initiatives, a study that reveals potential factors influencing the adoption of green initiatives may be helpful to fill the knowledge gap and provide opportunities for further research in this field. This type of study is relevant since the importance to integrate green initiatives within the company's business, as well as in the logistics service offerings, will probably attract even more managerial attention in the logistics industry in the future (Lieb and Lieb, 2010) and there will be a need of guidance. 


\subsection{Purpose ANd Research Questions}

Based on the previous discussion, the purpose of this licentiate thesis is to:

\section{Describe how different factors can affect the adoption of green initiatives among Logistics Service Providers, and how the adoption of green initiatives can be reflected in the service offering.}

In this thesis the term "factor" has been divided into triggers, drivers and barriers as well as firm characteristics, and in line with the main purpose, four further research questions have been developed and studied. First, in order to understand why LSPs have started to show interest and adopt green initiatives, an important step is to identify how different triggers can initiate the adoption of green initiatives. This clarification can facilitate the understanding of how the LSPs can progress further in greening their operations. This reasoning leads to the first research question, namely:

RQ1: How can different triggers initiate the adoption of green initiatives?

To clarify, in this thesis a trigger should not necessarily be associated with a driver. A trigger can be an incentive that "triggers a reaction" and activates or causes something to happen, while a driver stimulates or act as an impetus to activities or processes to move further. Therefore, in order to better understand what influences LSPs in their move towards a green development of their operations, the next step is to identify various drivers as well as barriers affecting LSPs in their adoption of green initiatives. Thus, the second research question is as follows:

\section{RQ2: How can different drivers and barriers affect the adoption of green initiatives?}

As mentioned in section 1.3, all companies may not be exposed to the same type of pressure or to the same extent (Lin and Ho, 2008). Therefore, it can be interesting to investigate how the perception of drivers and barriers might vary between different LSPs when adopting green initiatives. The disparity of perceptions can in turn depend on and be explained by the nature of the company and its characteristics. The third research question thus provides increased understanding of how firm characteristics can affect the adoption of green initiatives.

\section{RQ3: How can firm characteristics affect the adoption of green initiatives?}

The research questions presented above concern the LSPs' green performance throughout the entire company. In this thesis, green initiatives do not necessarily need to be a concrete green service offering to the customers. Instead, green initiatives can be a part of the general service offering or in some way support and affect the development of the service offering. Therefore, the last research question aims to consider in which ways the adoptions of green initiatives can be addressed towards the customers and be reflected in the LSPs' service offerings.

RQ4: In which ways can the adoption of green initiatives be reflected in the service offering? 
To conclude, in order to clarify the purpose and to facilitate for the reader, the connection between the main parts of this study and the research questions is illustrated in Figure 2 below:

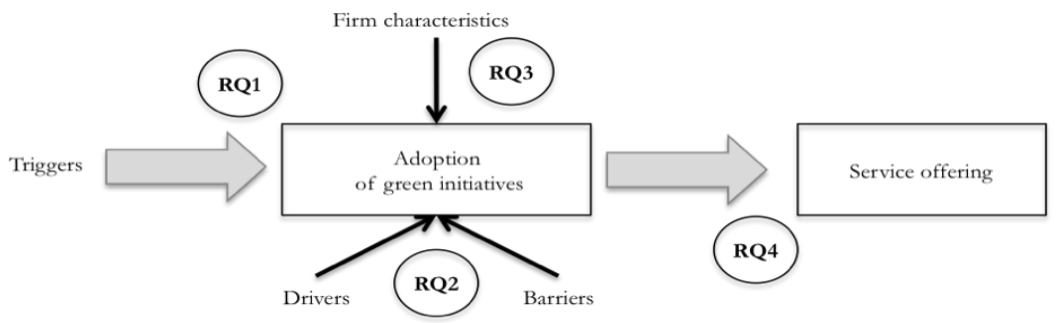

Figure 2 The position of the Research Questions.

\subsection{DEFINITIONS OF CENTRAL TERMS}

This section aims to discuss the central terms applied in order to ensure their clarity and consistent meaning throughout the thesis. This is essential in order to further specify and describe the focus of this licentiate thesis.

Logistics service provider (LSP) - The definitions of this term varies and an LSP can broadly be defined according to the Council of Supply Chain Management Professionals (CSCMP) as: "any business which provides logistics services. Includes those businesses typically referred to as 3PL, 4PL, LLP, etc. Services may include provisioning, transport, warehousing, packaging etc" (CSCMP, 2011). Furthermore, in this licentiate thesis, the activities of LSPs are in line with the definition of third party logistics proposed by Sweeney and Evangelista (2005): "Third-party logistics are activities carried out by a logistics service provider on behalf of a shipper and consisting of at least transportation. In addition, other activities can be integrated into the service offering such as warehousing and inventory management; information related activities, such as tacking and tracing; and value added supply chain activities, such as secondary assembly and installation of products".

Green initiatives - Since the development of environmental sustainability research in the logistics service industry still is in its infancy phase, there is not an explicit definition of green initiatives in the specific context of LSP. Hence, Martinsen and Huge-Brodin (2010) made an attempt in their article to group green initiatives into transport related measures (e.g. fuels, vehicle technology, mode choice, behavioural aspects, transport management) and beyond-transport initiatives (e.g. logistics system design, choice of partners, environmental management system, emissions and energy data). This approach has been applied in here.

Green service offering - As mentioned in the section 1.4 above, green initiatives do not necessarily need to be equated with green service offerings. Instead, in this thesis, a green service offering is a service offering with a more environmental focus, consisting of or supported by one or several green initiatives.

Trigger - A trigger, defined by the Merriam-Webster Encyclopaedia (2011) is something that acts like a mechanical trigger in initiating a process or reaction. However, in this 
thesis a trigger is equated with an incentive that "triggers reaction" and influences LSPs to start adopting green initiatives.

Driver - As mentioned earlier in section 1.4, a driver is not equivalent to a trigger in this thesis. According to the Merriam-Webster Encyclopaedia (2011), a driver is defined as something that provides impulse or motivation. The interpretation of a driver in this thesis is as follows: arguments (e.g. cost reduction, increased competiveness or economic means of control) or influences from stakeholders (e.g. customers, employees or top management) that stimulate or drive the adoption of green initiatives and thus push LSPs' green development processes to move further.

Barrier - The term barrier can be defined as something that impedes progress or achievement (Merriam-Webster Encyclopaedia, 2011). In the context of this thesis barriers can be arguments such as high investments costs, lack of financial resources or lack of customers interest, which hinder the process of adopting green initiatives within LSPs. Hence, it is worth mentioning that distinguishing between a driver and a barrier often lies in the eyes of the beholder. This aspect and how this may have influenced the empirical data collection has been taken into consideration in the analysis of the drivers and barriers.

\subsection{SCOPE AND FOCUS OF THE LICENTIATE THESIS}

The main focus in this thesis concerns how different factors can affect the adoption of green initiatives among LSPs and how the adoption of green initiatives can be reflected in the service offering. As mentioned earlier, existing research from a LSPs' perspective is not very well established, and especially not from a green context. Since LSPs are in the infancy phase of their green development, this leads to some limitations in the scope and focus of this research.

This study does not aim to give a comprehensive picture of LSPs' green situation; instead it intends to give a "snapshot" of how it looks today. However, the case companies studied have been selected since they have shown a more proactive approach when it comes to integrating green thinking into the company, as well as adopting green initiatives. It is worth mentioning, that whether or not the green initiatives mentioned by the studied LSPs actually lead to a decreased impact on the environment and reductions of carbon emissions, will not be further explored and is outside the scope of this thesis.

Furthermore, this research does not aim to give a comprehensive spectrum of all the drivers and barriers that can possibly affect the adoption of green initiatives. Instead, it provides a selection of the most commonly mentioned and highlighted drivers and barriers in the literature, as well as those influencing factors stressed by the case companies, in order to allow an investigation of these factors' impact on the adoption of green initiatives.

Moreover, the aim of this research is to create a link between the two fields, logistics and marketing, by explaining how the adoption of green initiatives can be reflected in the service offerings provided by LSPs. Therefore, this research intends to contribute to a 
better understanding of the link between LSPs environmental work and their service offerings.

\subsection{THE OUTLINE OF THE LICENTIATE THESIS}

First, it is essential to clarify that this licentiate thesis is a compilation which include four different papers. Therefore, the main parts of this thesis (hereafter referred as the Thesis Frame), attempt to link these four papers to each other. This means that the Thesis Frame becomes more or less independent of the various papers. However, it is important to note that in some cases, it may be worthwhile to review the papers a little bit closer in order to get a more detailed picture of a particular area. However, in order to give the reader a quick overview of the structure of the licentiate thesis, this section will provide a brief review of the chapters included and their main contents, see Figure 3 below.

\section{Chapter 1: Introduction}

The thesis starts with a background that highlights and addresses how the logistics service providers' industry has evolved during the last few years and in what way this industry has been affected by the ongoing green agenda in our society. This background leads then into the purpose and the research questions of the thesis. In addition, this chapter also presents the scope and focus of the study as well as definitions of central concepts and terms, and discusses how they will be used in this thesis.

\section{Chapter 2: Theoretical Framework}

This chapter begins with a brief presentation of literature concerning logistics offerings in general. This is followed by a presentation of green initiatives within the LSP industry as well as an identification of different kinds of influencing factors, such as drivers, barriers and firm characteristics, which may have some impact on the adoption of green initiatives.

\section{Chapter 3: Research Approach}

The third chapter describes the methodology of this study, and includes sections such as research design and research methods. The relevant research methods in this thesis are literature reviews, a case study and a questionnaire survey. For each of these methods, a detailed description is provided in order to inform the reader about how these methods have been applied in this study.

\section{Chapter 4: Summary of the Appended Papers}

As mentioned earlier in this section, the licentiate thesis is based on four papers. A summary of each of the appended papers is presented in this chapter in order to give the reader an insight into the different papers.

\section{Chapter 5: Analysis}

The analysis in this thesis is organised and structured according to the research questions developed that were mentioned earlier in this chapter. The analysis is mainly based on the findings from the presented papers above, but additional input from the comprehensive case study report (Appendix 2) and the questionnaire survey has inspired the analysis work. 


\section{Chapter 6: Conclusions and Further Research}

This final chapter discusses and presents some main conclusions from this study. The contributions are presented as well as suggestions and directions for further research.

\section{Appendices}

The appendices attached to this licentiate thesis include first and foremost the four different papers. In addition, the case study report (including detailed descriptions of the 6 different case companies), the survey questionnaire as well as the interview guide can also be found among the appendices. In order to give the reader a quick overview of the appended papers, the title and the purpose of each paper is listed below.

\section{- Paper 1 - Developing Sustainable Logistics Services}

This paper aims to present a framework for how to consider sustainability in the development of logistics services. In addition, the paper aims to develop a research agenda for further research needs regarding how logistics companies can include environmental aspects in their service offerings.

- Paper 2 - Green Initiatives in the Transport and Logistics Service Industry: An Exploratory Case Study of Logistics Service Providers

The purpose of this paper is to explore and describe the awareness and adoption of green initiatives among LSPs, as well as identify drivers and barriers affecting the green initiatives undertaken by LSPs.

- Paper 3 - Understanding Efficiencies Behind Logistics Service Providers' Green Offerings

The objective of this paper is to indicate where green-labelled LSPs are positioned today in their development of green service offerings. Furthermore, the paper seeks to explain the underlying rationale behind the development of green service offerings.

- Paper 4 - The Challenge and Adoption of Green Initiatives for Transport and Logistics Service Providers

Finally, the last paper aims to develop a base for further investigations into green initiatives carried out by LSPs, and analyse if the green initiatives implemented are dependent on the firm characteristics of the LSPs, as well as examining the drivers and barriers experienced. 

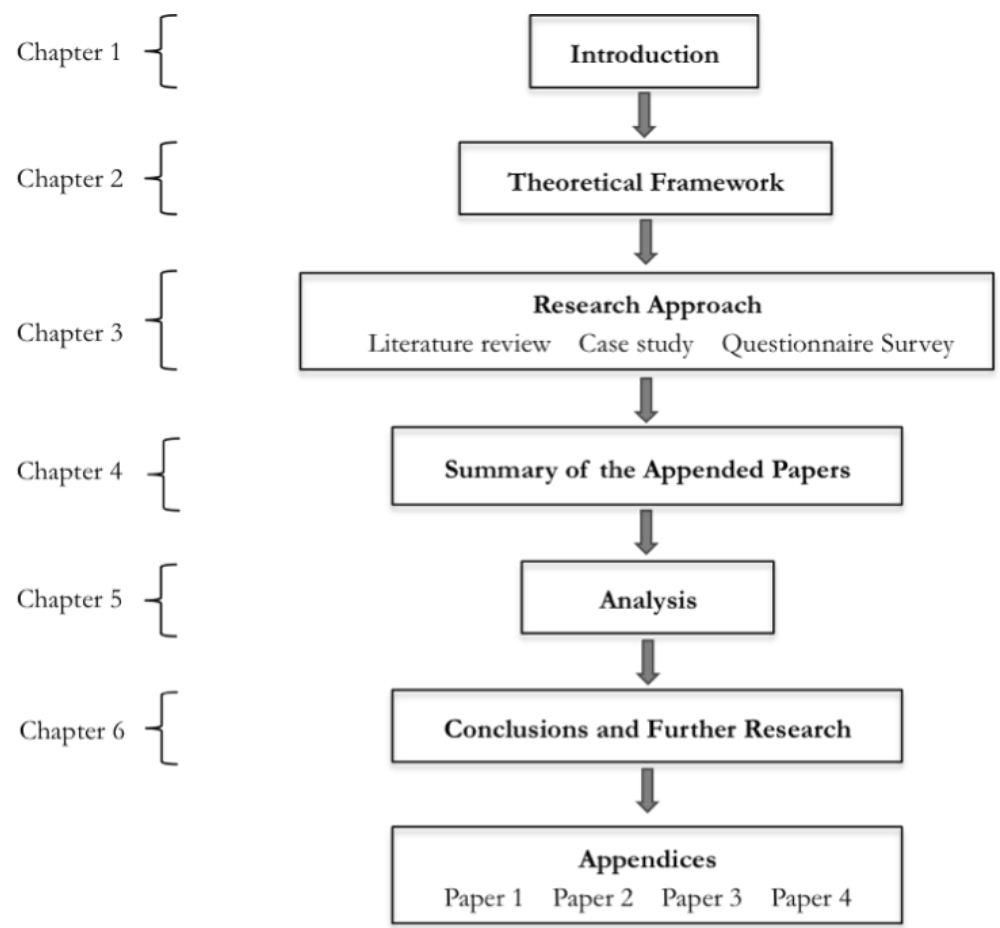

Figure 3 The outline of the Licentiate Thesis. 


\section{THEORETICAL FRAMEWORK}

This chapter presents at a general level the development of logistics offerings as well as suggestions for green initiatives to be undertaken by the LSP industry. Furthermore, the chapter also identifies some factors, i.e. drivers, barrier and firm characteristics, that may affect LSPs when adopting green initiatives within the company. Deeper theoretical briefings regarding the addressed areas in this Thesis Frame can be found in the appended Papers (see Appendices 4, 5, 6, 7)

\subsection{LOGISTICS OFFERINGS IN GENERAL}

As a result of increased globalization of businesses, in addition to increased pressure to achieve competitive advantages, the need of logistics services among manufactures and trading companies has increased significantly. The primary aim of this is for companies to be able to introduce products and service innovations more quickly to the market.

In general, the LSP industry can be described and ranged from several players that offer basic transport services to a few market leaders that offer a wide range and scope of logistics services (e.g. one-stopping shopping) and niche firms with a more diversified portfolio of interest (e.g. mentioned by Carbone and Stone, 2005; Larson and Gammelgaard, 2001; and Dobie, 2005).

In the logistics literature, several researchers have been studying the contents of logistics services and how different types of LSPs operate (e.g. Berglund, 2000; Bagchi and Virum, 1998; Murphy and Poist, 2000; Hertz and Alfredsson, 2003 and Lai, 2004). The service variety provided by LSPs mentioned in the literature is broad. For example, Yu et al., (2010) conclude that beyond traditional logistics functions like transportation and warehousing, a LSP can also provide other value added services, such as inventory management, logistics coordination, carrier selection, reverse logistics, supply chain management integration, freight forwarding, rate negotiation, electronic funds transfer, fleet management/operation, product assembly and kitting, spare parts fulfilment, marketing services, security services, project management, logistics information and IT, contract manufacturing and management of call centres. These listed examples demonstrate the width of the LSP industry's offerings.

Even if several companies focus on standard solutions and economy of scale, many LSPs have taken initiatives to broaden the scope of their services (Murphy and Daley, 2001). This growing interest and development towards more customised services arise upon the desire to fully satisfy the increasing requirements of customers (Berglund et al. 1999; Mortensen and Lemonie, 2007) and the willingness to improve customer service levels (Daugerty et al., 1992). By the transformation of scope and characteristics of LSPs service offerings this has led to a development of new service strategies. These new service strategies are affected by the original capabilities base and this may have a strong influence on further development stages of the company (Evangelista, 2011). The trend towards more value-added services give LSPs the opportunity to differentiate their business towards their competitors (Evangelista, 2004), as well as strengthen the relationships with the customers (Skjoett-Larsen, 2000). It is a shared opinion that the ability to position the company and its services can help LSPs in directing the company's 
efforts and thus generate a strategic advantage (e.g. Juga et al. 2011; Hertz and Alfredsson, 2003 and Lai et al., 2002).

In a study conducted by Lai (2004), differences in service performance among different types of LSPs were examined. The results from the study singled out a number of logistics services and suggested that there are four types of LSPs. The first type was labelled Traditional freight forwarders due to its low capability to carry out value added and technology-enable logistics services. The second group, Transformers, which also was the largest of the LSP types, possessed a high level of capability in freight forwarding and technology-enabled logistics service and a medium level of capability to perform valueadded logistics services. These actors also seemed to make an effort to expand their service offerings and move towards a more comprehensive LSP. The third type of LSP identified, Full service providers, had a high level of capability in all of the three logistics service factors, which suggested that they are comprehensive LSPs with the capability of providing a wide range of logistics services. The last and the smallest type of LSPs, Nichers, showed a weak potential to carry out freight forwarding logistics services but possessed a higher level of capability to carry out value-added and technology-enable logistics services.

Due to the development towards more customised services, the pressure on the LSP industry has been characterised by more strategic influences in terms of market coverage, improving the level of service and increased flexibility regarding changing requirements of customers (Hertz and Alfredsson, 2003). For example, LSPs have lately faced new kinds of requirements from customers concerning green initiatives, and the following sections present how LSPs have responded and can be affected by these requirements.

\subsection{GREEN INITIATIVES WITHIN THE LSP INDUSTRY}

Research in green supply chain management (GSCM) has significantly expanded over the last decades in connection to the growing importance of the environmental components in the management of supply chains. In general, the green supply chain (GSC) is a broad concept that includes different approaches by which companies work with their supplier and/or customers in order to improve the environmental performance of their operations. Two primary goals of GSC are identified by Lu et al., (2007, p. 4 317):

1) Consistently meeting specified environmental performance criteria among the participants in the supply chain and promoting responsible corporate environmental behaviour among all the players in the chain of products and services, and;

2) Helping suppliers to recognize the importance of resolving environmental issues and support them in installing their own improvement initiatives.

However, there is not an established definition of GSCM initiatives in the current literature and as indicated by Sarkis (2006), the boundary of GSCM is strictly dependant on the type and scope of initiatives adopted by companies participating in supply chain processes. For example, Zhu and Sarkis (2004) argued that in the literature, GSCM initiatives range from green purchasing to integrated green supply chains flowing from supplier to manufacturer to customer, and include reverse logistics. Furthermore, Eltayeb 
and Zailani (2009) suggested a general classification of green supply chain initiatives organised into the following three categories: eco-design or design for the environment, green purchasing and reverse logistics. In addition, Porter and van der Linde (1995) advocate that investing in greening initiatives is both eco-friendly and business-friendly as it increases business efficiency through resource savings, waste elimination and productivity improvement. The authors also stress that such initiatives may also lead to major competitive advantages in innovation and operations.

This identification of green initiatives may also be relevant for the logistics transport industry. In the last few decades, LSPs have gradually transformed the scope of their service offering shifting from providing single-activity toward a business model based on offering a wider range of services (Ashenbaum et al., 2005). As result of this evolving process, LSPs are in the position of adopting both green transport and non-transport related initiatives or a mix of both altogether. Secondly, LSPs may adopt an approach based on designing an integrated package of initiatives to improve the environmental sustainability of service they provide to their customers.

Although the body of literature on GSCM is growing, little research has been conducted on environmental issues in the logistics service industry, but it seems it has expanded over the last few years (Wolf and Seuring, 2010). In the literature, there is not an explicit definition of green initiatives in the specific context of LSP; however two recent papers offer a categorisation of green initiatives. On the basis of the survey, Lieb and Lieb (2010) conducted a group of 28 CEO of large LSPs operating in the North American, European, and Asia-Pacific, where they clustered green initiatives into four categories: administrative, analytical, transportation-related, and a broadly defined "other" category. Similarly, Martinsen and Huge-Brodin (2010) grouped green initiatives into transport related measures (e.g. fuels, vehicle technology, mode choice, behavioural aspects, transport management), and beyond-transport initiatives (e.g. logistics system design, choice of partners, environmental management system, emissions and energy data) based on a review of general green logistics literature, a survey, and a scan of company homepages.

Adoption of green initiatives is a great challenge for logistics service providers that strive to develop and implement more green service offerings towards their customers. In order to get a better understanding of some of these challenges, the following two sections present different factors that may affect the adoption of green initiatives among LSPs.

\subsubsection{Drivers and barriers for green initiatives}

This section presents some previously identified drivers and barriers that may affect LSPs when adopting green initiatives. Some of the factors can both be viewed as a driver and as a barrier and also both have an internal as well as an external effect on the adoption of green initiatives.

In order to mitigate detrimental environmental effects, logistics and transport activities (e.g. hazardous goods) have become subject to more regulation. Transportation provides a good example as this activity causes a high rate of negative environmental impact such as pollution (McKinnon, 2006). According to Walker et al. (2008) government regulation and legislation is a major driver of companies' environmental efforts. In addition, Wong et al. 
(1996) highlight the importance role of government and their environmental strategies as well as how this influences the consumer behaviour. Government legislation was one of the biggest drivers of corporate sustainability investment according to a study conducted by Berns et al. (2009). However, compliance with environmental legislation is no guarantee of improved environmental performance; indeed it could also be seen as barrier due to weak enforcement (Shi et al., 2008), low levels of awareness and the absence of a central source of information (Balzarova and Castka, 2008). Also, Roth and Kåberger, (2002) stress the complexity to translate general environmental targets to specific requirements for a single company.

Another pressure that LSPs must consider from the sustainability point of view, relates to reduction of transportation costs due to rising fuel prices (van Hoek and Johnson, 2010). This encourages LSPs to implement sustainable cost-cutting initiatives such as the adoption of transport network optimization software. Many articles in the literature notice the desire to reduce costs as a common driver for environmental work (e.g. Walker et al. (2008), van Hemel and Cramer, (2002), Salomone (2008)). Companies that excelled in various green supply chain activities are often driven by a strong focus on cost savings, waste elimination and quality improvement (Walker et al., 2007). However, economical and financial aspects are often seen as barriers when companies try to meet the increased environmental demands from customers (e.g. Dahlman et al., 2008, Balzarova and Castka, 2008 and Shi et al., 2008). Lack of financial resources, difficulty to specify the expected results and translate them into economical terms, as well as low returns are some factors mentioned in the literature. In addition, according to a study aiming to identify the main barriers to the uptake of environmental technologies in the freight transport sector, it was shown that long investment periods were also cited as a barrier to the replacement of traditional technologies with more environmentally friendly ones (ETTAR project, 2007).

Other barriers, commonly mentioned in the literature regarding environmental work are technical knowledge and information. Technical knowledge can both be an internal and an external barrier and includes: limited in-plant expertise, lack of technical training, lack of access to external technical support (Shi et al, 2008), uncertainty regarding existing techniques and inability to eliminate some risks or effects (Post and Altman, 1994). Like technical knowledge, information can also be viewed both as an internal and external barrier when it comes to communicating and spreading environmental information and knowledge within the company as well as to its customers. In addition, information barriers can also include difficulties such as accessing and collecting appropriate environmental data (Post and Altman, 1994).

Environmental considerations can have an impact on several logistics decisions along the supply chain such as location, sourcing of raw material, modal selection and transportation planning (Wu and Dunn, 1995). Efforts towards greener logistics require the extension of traditional economic supply chain objectives to include ecological objectives. This increases complexity in the management of the logistics business, creating additional costs and limiting options. Managerial and organisational barriers are commonly mentioned in the literature in relation to the adoption of environmental initiatives. These barriers include management resistance to change (Shi et al., 2008), lack of understanding and awareness from the top management and attitudes of employees (Post and Altman, 
1994). However, management and employees can also be seen as drivers. According to Berns et al., (2009), employee interest in sustainability can enhance staff recruitment, retention and engagement and other employee-related issues. This can be seen as major benefits when addressing sustainability. Furthermore, operational and environmental improvements have found to be positively related to employee involvement (Walker et al., 2008).

The adoption of green initiatives is a great challenge for LSPs that are seeking to develop and implement more sustainable service offerings. In order to successfully integrate sustainability dimension into business processes, companies must connect the sustainability dimension to the business strategy and link these aspects to both short-term and long-term financial performance. Berns et al., (2009), for example, claim that most companies appear to lack an overall plan for managing sustainability and delivering results. In addition, Seidel et al., (2009) mention that implementing environmental work can lead to an opportunity to develop new innovations. In other words, LSPs may consider adoption of green initiatives within the company as well as in their service offerings as a potential source of revenue or cash flow.

Pressure from customers is seen as a dominant driver in the literature when implementing environmental work in the company (e.g. Foster et al., (2000) and Berns et al., (2009)). Hence, in what ways and how much customers drive green supply chain management projects varies significantly (Walker et al, 2008). But as a result of increased investment in order to accomplish environmental objectives among manufactures and retailers, they may expect LSPs to improve the sustainability of their operations in order to support the environmental strategies of their customers. However, customers are also mentioned as a barrier in the literature and Shi et al., (2008) point out that market barriers can include such as lack of preferences and demands from customers as well as weak public awareness and pressure. Another source of pressure, affecting a LSP to implement environmental work, can come from competitors that already have started to adopt environmental policies. According to a study conducted by Salomone (2008), reasons such as greater competitiveness on the market and being able to exploit new market opportunities have some significance in motivating companies to integrate environmental aspects into their activities. Furthermore, companies may even aim to be the environmental leader on the market in order to win new market shares and Henriques and Sadorsky (1999) also stress other benefits such as be able to set industry norms and/or legal mandates as well as to have the ability to drive environmental innovation.

Pressure from suppliers can also be taken into account as driver when LSPs adopt green initiatives within their companies. In contrast, it turned out in a study conducted by Salomone (2008) that no firm cited pressure from suppliers as a driver for implementing environmental work. Walker et al. (2008) also claim that there is a lack of previous research that identifies suppliers as a key driver of environmental supply chain management practices. In addition, an increased pressure from investors can also be classified as a driver for companies to start implementing environmental work within the company (Walker et al., 2008). 
Furthermore, LSPs may be concerned with how the company and its brand are perceived by the society and therefore be affected by e.g. pressure from public authorities (Salomone, 2008) or negative media attention caused by environmental action groups (van Hemel and Cramer, 2002). As described, many different factors can influence companies in their greening, both as drivers and barriers. Although the identification of these factors is based on different companies, they appear as relevant also for LSPs.

\subsubsection{Firm characteristics influencing on green initiatives}

The influence of firm characteristics on the adoption of green initiatives undertaken by LSPs has been studied in recent literature. In general, size (in terms of both employees and turnover) is one of the most important firm characteristics expected to influence the adoption of green initiatives. The works of Lin and Ho (2008) and Ho et al. (2009) have shown that number of employee, company history, and capital size influence green initiatives taken by LSP companies.

Furthermore, Evangelista et al. (2010) indicate in their paper that there are some differences between small and large LSPs. For example, large 3PL companies show a higher level of awareness of environmental sustainability in comparison with smaller ones. This is particularly true for the strategic prioritisation of environmental issues and the role of customers in driving green initiatives. With reference to the adoption of green initiatives, the results reveal that large LSP companies tend to adopt a set of coordinated sustainable initiatives both in transport and beyond the transport area. A different approach has been detected between small LSPs that show a focus on reducing the environmental impact of transport activities only.

The literature does however neglect to analyse the relationship between green initiatives and other important firm characteristics such as the type of product shipped and type of service provided. Hence, the purchasing literature indicates that companies in different industries experience different hindrances and drivers which lead them to apply different practices (Zhu and Sarkis, 2006), suggesting that the drivers and barriers experienced by LSPs can be dependent on characteristics such as type of product and service provided. 


\section{RESEARCH APPROACH}

The purpose with this chapter is to describe the research approach taken for the research presented in this licentiate thesis. First, the overall research design is presented. This is followed by the selected research methods, which are literature reviews, a case study and a questionnaire survey. After that, the analyses of the research questions are discussed. This chapter ends with a subsection that includes the researcher's reflections upon the actual research process, a brief presentation of the research project of which the research has been a part as well as the researcher's contributions to the appended papers in this licentiate thesis.

\subsection{RESEARCH DESIGN}

In general, a research design is the logic that combines the data to be collected to a study's initial research questions. Before deciding upon the most appropriate research design or data collection methods, the type of research to be conducted should be clarified. This is necessary since the existing knowledge within the research area affects the type of research that is conducted, which in turn influences the selection of suitable data collection methods (Yin, 2009). The research in this licentiate thesis is explorative in its nature. An explorative study is used in situations where limited prior knowledge and research exist, and aims therefore to find a basic understanding of the current scenario. This approach seems appropriate for this licentiate thesis since, as described in Chapter 1, LSPs are in the infancy phase of the development to adopt and integrate green initiatives into their business and service offerings. Therefore, there is a need for research which to investigates the current situation and the underlying mechanisms (in form of triggers, drivers and barriers) that influence the LSPs in their continuing work to adopt green initiatives.

Due to the explorative starting point of this licentiate thesis, the research approach has been inspired by an abductive reasoning described by Kovács and Spens (2005). Applying an abductive research process starts at the point where observations in the empirical research do not match prior theories, and an iterative process starts in an attempt to find new matching frameworks or to extend the theory used prior to the observation. In other words, this type of research approach aims to understand a new phenomenon and to suggest new theory in the form of new hypotheses or propositions. Therefore, the research design of this licentiate thesis takes its starting point in initial insights based from prior observations and knowledge, followed by an iterative process of travelling back and forth between relevant and emerging literature (literature reviews) and empirical data collected from case studies and a survey study. This was done in order to build up a knowledge base within the field with the aim to provide more generalised descriptions and potential explanations to the subject studied. The overall research design is illustrated below, see Figure 4. 


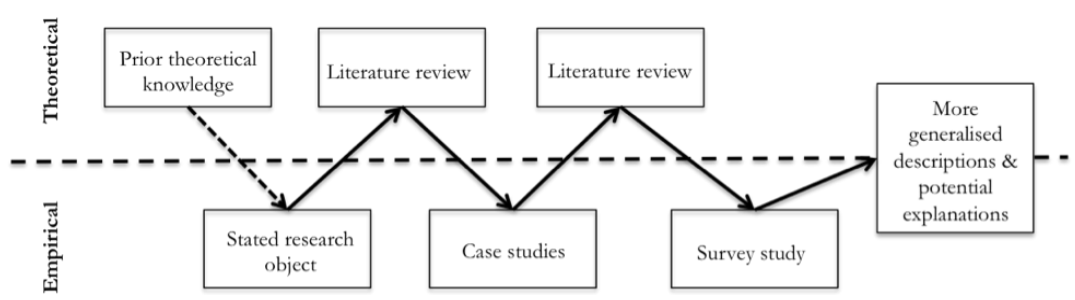

Figure 4 The overall research design process.

\subsection{RESEARCH METHODS}

In this licentiate thesis, three different research methods has been used namely, literature reviews, a case study and a survey study.

As illustrated above in Figure 4, the first research method applied was a literature review which consisted of a structured review of the relevant literature. This research method has been used during the whole research process in either a structured or in a more iterative process. The second research method applied was case studies. This was followed by a survey study. The use of this survey in this licentiate thesis was partly to test and validate the findings from the case studies. Below, Table 1 shows which of the research methods are applied in each of the appended Papers.

Table 1 Research methods applied for the appended Papers.

\begin{tabular}{l|ccc} 
& Literature Review & Case Study & Survey study \\
\hline Paper 1 & X & & \\
Paper 2 & X & X & \\
Paper 3 & X & X & X \\
Paper 4 & X & &
\end{tabular}

A more detailed description of the three different research methods used in this thesis is presented below.

\subsection{LiterATURE REVIEW}

Literature reviews play a critical role in doctoral thesis and journal publications, and are also highlighted for the potential role they could play in creating and building bodies of knowledge and informing policy and practice (Tranfield et al., 2003). One way to do this could be to conduct a systematic review. However, a systematic review should not be regarded as a traditional literature review since it differs from other review methods because of its distinct and exacting principles (Denyer and Tranfield, 2009). Even if a systematic review approach has not been applied here, the systematic review thinking has inspired some of the literature reviews conducted. These structured and more systematically inspired literature reviews, include careful selections of relevant keywords and well-documented information about the number of total and relevant hits. 
Two more structured literature reviews have been applied in this thesis. Paper 1 is based upon the first of these. This review addresses service and product development with environmental considerations. Since no article in this search described the logistics company's point of view, the main literature review was complemented with a second review to include the perspectives of the logistics companies. The reviews aim to describe the findings from these two areas separately, as well as to adapt the findings on sustainable product and service development to the setting of a logistics service provider.

The second structured literature review was necessary to develop a framework which describes which barriers and drivers an LSP could face in adopting green initiatives. Other literature reviews have also been carried out this research process. An iterative process was used for these reviews, where for example, a snowball approach of relevant references has been used.

The areas of literature taken up in the different papers as well as in the thesis's frame of references are: green supply chain management, green logistics, general literature on the LSP industry and its development, and marketing and service literature, both with and without green considerations.

Disciplines from different fields, in terms of concepts, principles, methodologies and approaches, can be applied to various logistics issues, problems and opportunities (Stock, 1997). For example, the author stresses how the logistics field can be built on theories borrowed from other disciplines such as marketing (e.g. buyer behaviour and segmentation), business/management and psychology (e.g. perception, learning theory and network model). This approach of using marketing literature and concepts in order to fill the gap in the logistics literature can be appropriate since the research area studied here is close to the interface between logistics and marketing. However, the link between logistics and marketing has not totally been neglected in the existing literature. For example, Mentzer et al. (2001) highlight the importance of a close interrelationship between marketing activities and logistics activities, which in turn indicates that these two areas should not only be considered separately. Therefore, by applying marketing literature in a logistics context this could facilitate explaining underdeveloped areas within the logistics literature. Marketing literature may also serve as a platform to get a deeper understanding of the underlying mechanisms regarding LSPs' approaches towards green initiatives reflected in the service offerings.

\subsection{CASE STUdies}

Case research methodology is both appropriate and essential when there is a lack of theory or when theory exists but the environmental context is different (Stuart et al., 2002). One of the strengths of case research is that the method can be used in early, exploratory investigations where the variables are still unknown and the phenomena not well understood (Meredith, 1998) and it can also generate research ideas and hypotheses that are worth pursuing further (Handfield and Melnyk, 1998). Therefore, the case study approach seems suitable since the topic of this licentiate thesis is still in the understanding, discovery and description stage. Meredith (1998) also highlights that case studies can develop understanding, especially in a field where the subject matter is very complex. Both the novelty and the complexity criteria match the situation for LSPs and 
their offerings in general, as well as for the existence of possible green service offerings. In the literature, case studies are described as based on any mix of quantitative and qualitative evidence and can include different kinds of data collection methods such as interviews; both structured and semi-structured, critical incident techniques, surveys, observations and focus groups (Fitzgerald and Dopson, 2009). In this licentiate thesis, the case studies are based on qualitative data and have mainly been collected through interviews.

\subsubsection{Selection of cases}

Since case-based research depends on investigative observations, the selection and number of firms studied is an essential and important step in the case study process. The selection of appropriate cases can facilitate controlling extraneous variation and also to help define limits for generalizing the findings (Eisenhardt, 1989). The decision whether to conduct a single case study or a multiple case study depends to a large extent on the aim of the research. Single-case studies can richly describe the existence of a phenomenon (Siggelkow, 2007) while multiple cases enable a broader exploration of research questions and also a clarification of whether the finding is idiosyncratic to a single case or consistently replicated by several (Eisenhardt and Graebner, 2007). Here, a multiple case study has been conducted with a set of six case companies. Yin (2009) argues that, in most situations, six to 10 cases should provide evidence to support or reject propositions, while Eisenhardt (1989) recommends four to ten cases, which means that the selection of six cases used in this licentiate thesis falls within these recommended ranges. All the case companies were LSPs, active on the Swedish market and the companies were selected on the basis of the following criteria:

- Some demonstrable ambition regarding green initiatives, and also certain external recognition for this (either from the news media or previous contact with the research community)

- Active on the same market, thus facing a similar environment with regards to market demands as well as rules and legislation for transport related to green issues. The choice of the Swedish market was selected due to the researcher's familiarity with this market and ease of access to the case companies

- Varying size, profile, geographical coverage and also a varying range of logistics service offerings in order to give a width to the empirical data.

In order to demonstrate a study's internal validity, a researcher needs to record evidence of other factors that might be alternative explanations for the observed patterns (Stuart $e t$ al., 2002). To maintain internal validity, the last criteria mentioned above (e.g. varying size, profile, geographical coverage) aims to outline if the patterns observed vary in terms of the scale and scope of the studied LSPs.

The selection process identified two medium sized LSPs with local/regional business and a wide range of transport and basic logistics services (localised in different regions); two small/medium size LSPs specialized in express deliveries (one independent and one, part of a larger Nordic corporation); and finally two large international LSPs with a wide range of service offerings (whose philosophy regarding the inclusion of environmental issues differ substantially). In order to encourage openness of response during the data 
gathering process, it was agreed that company names would remain anonymous, and the case companies were thereby labelled Alfa, Beta, Gamma, Delta, Epsilon and Zeta.

\subsubsection{Descriptions of cases}

This section provides a brief description of the different case companies. For further information and a more detailed case presentation, see Appendix 2.

\section{Company Alfa}

Alfa is one of the largest transport intermediation companies in Sweden. The company has about 140 subcontracted carriers who are also part owners in the company, which leads to more than 500 people being employed in the entire concern. Over $90 \%$ of Alfa's services cover the full range of those available within the transport market with the exception of refrigerated transport. (Added services are offered as a complementary to all transport services depending on the demand.) The customers of the company consist mainly of industries and municipalities, but the company also has also end users as customers.

\section{Company Beta}

Beta is one of the largest logistics and transport companies in the northern part of Sweden. The company has approximately 260 subcontracted carriers, also part owners of the company, and this gives a total number of 1000 employees in the entire concern. Beta handles everything from gravel and industrial goods to sensitive consumer products and temperature-sensitive foods. The operations mainly concern FTLs. Beta cooperates closely with its customers to develop complete logistics solutions.

\section{Company Gamma}

Gamma is a franchise-based express transportation company with access to 700 vehicles and an extensive air route network. The company provides systemised and customerspecific door-to-door express delivery solutions for time critical and high value products within the field of high-tech, automotive spare parts and medical equipment. Packages are transported either by road or air depending on where they are headed, how large they are and how urgent the delivery is. The main group of customers are businesses, but express deliveries to private customers rarely occur.

\section{Company Delta}

Delta is a specialist in the express and delivery transport area and is part of the bigger Nordic company active in the postal and logistics industry. Their range of service offerings includes delivery, distribution, express and home delivery. The company offers different kinds of transportation modes such as air, road or track. Delta works mostly business-to-business, even though in some cases the company delivers directly to private consumers.

\section{Company Epsilon}

Epsilon is part of one of the world's leading logistics groups and offers integrated services and customised solutions for managing and transporting mail, goods and information. The company offers the whole spectrum of transportation modes such as air, road, track and sea. The main customer target group consists of the metal- and telecom industries. 


\section{Company Zeta}

Zeta is also a part of one of the world's largest logistics companies and is able to provide land transport within Europe, global ocean- and airfreight and customised logistics solutions. The company offers everything from rail transport to parcel transport, and offers logistics services such as warehousing and goods handling. The company does not offer courier or mail freight. The split between inputs for industry and consumer products is about $50 \%$ each.

\subsubsection{Case data gathering}

The case descriptions are mainly based on semi-structured focused interviews, which according to Yin (2009) are suitable for explorative studies. These kinds of interviews are also recommended by Stuart et al., (2002) since they allow some flexibility regarding the data-gathering which ensures to uncover all of the pertinent data when companies with different characteristics and competences are being surveyed. During the interviews, a data collection guide was used (see Appendix 1), as is suggested by Yin (2009) in order to ensure the research reliability, especially in a multiple case study.

The data collection guide was divided into three parts, covering the following areas:

- A general picture of the company and the range of services offered;

- Factors (in form of drivers and barriers) affecting the company's green initiatives and existence of green service offerings (the respondents were also asked to specify and to comment on the impact of each factor on a scale of 1 to 5 , where 5 had the greatest impact, later translated into a scale with a range of low (1-2), medium (3), high (4-5)); and,

- How environmental issues can be integrated into the price picture of these green service offerings.

This was sent to each respondent in advance and suggested as an outline for the interview to give respondents an opportunity to reflect upon and look for answers to questions they knew less about. All of the respondents had good knowledge of the company's environmental efforts and were either environmental managers or involved in and responsible for sustainability activities at the company. The information was obtained from telephone interviews with one representative from each company. Each interview lasted for at least one hour, and in some cases, further contact with the company's representative was obtained through company visits, telephone and email. All the interviews were taped and transcribed and the respondents were given the opportunity to reflect and comment on the transcriptions, which three of the respondents did. According to Yin (2009) documenting the interviews can increase the study's reliability and giving the respondent an opportunity to review the draft from the conducted interview can lead to strengthening the construct validity of the case studies.

Besides the interviews, secondary data such as information about the companies was collected from a variety of information sources including company reports and websites. This kind of information was used to enhance and explain the information collected during the interviews. In this way, information was triangulated across data sources, 
which is an appropriate method suggested by Stuart et al., (2002) for ensuring the construct validity of the case study.

\subsection{THE SURVEY}

In order to broaden the scope as well as strengthen the findings from the case study, the researcher planned to conduct a questionnaire survey. However, since collaboration with a researcher at University of Naples Federico II, Italy had begun at this stage in the research process, a joint decision was taken to construct a questionnaire survey together. This was done since the research area at the different sites seems to coincide, which awakened an interest to carry out a comparative study between Sweden and Italy. During the research process, a number of other European universities have showed interest and joined the study in order to conduct similar work in their own countries. Besides the two mentioned above the academic research institutes involved in this survey process are: Dublin Institute of Technology (Ireland), Heriot-Watt University (United Kingdom) and Tampere University of Technology (Finland).

\subsubsection{Preparation of the questionnaire survey}

The questionnaire is derived from the researchers' earlier research projects based on case studies and literature reviews. Data and information collected through the questionnaire allow the identification of green initiatives, influencing factors (drivers and barriers) as well as the basic characteristics of the companies studied. Before launching the survey, the questions and answer alternatives were discussed with other academics and also pre-tested with potential respondents in Sweden, through a test-survey. This pre-test of the questionnaire resulted in some changes in wording and clarifications as well as the reduction of the number of questions since the questionnaire was perceived to be quite long and comprehensive.

\subsubsection{Design of the questionnaire survey}

For the Swedish market, a web-based survey was used. The questionnaire was created and was sent out to the respondents using the survey-tool Webropol. The web-based survey approach was chosen instead of the traditional mail or postal survey approach, since it speeds up and facilitates the data collecting process as well as being simple and less time consuming for the respondents. Grant et al., (2005) mention other advantages of using Web-based surveys such as observing recording question answers, providing support to the respondents if necessary, digitization of information which leads to savings in both time and money and also facilitating the process to send out reminders, if the response rate does not prove to be satisfactory.

The scope of the questionnaire is quite extensive in its nature (see Appendix 3) and allows for the identification of green initiatives, influencing factors (in the form of drivers and barriers) as well as firm characteristics of the companies studied. The questions included were drawn directly or indirectly from the researchers' earlier literature reviews or results from case studies, which in turn, ensures the content and construct validity of the questionnaire. The design of the questions composed a mixture of closed and open-ended questions as well as different kinds of scales. The scales consisted mainly of a five-degree Likert scale, ranging from 1 (totally disagree) to 5 (totally agree). The questionnaire was divided into five sections with the following contents: 
- Company profile - these questions addressing firm characterises were mainly of a classifying character. Aspects targeted were size (number of employees and annual turnover), geographical distribution, type of services provided and position in the supply chain.

- Adoption of green initiatives and resources - this section aimed to investigate the adoption of green transport and supply chain related measures, the involvement of the different functions of the company in green initiatives and the adoption of ICT applications to manage the environmental impact of transport and logistics operations.

- Drivers of green logistics initiatives - a variety of different arguments' impact on adoption of green initiatives were addressed as well as the influence of different stakeholders.

- Barriers to green logistics initiatives - the barriers investigated were divided into internal and external barriers affecting the adoption of green initiatives.

- Future prospect for adoption of green initiatives - the respondents were also asked to answer questions about what green transport and supply chain initiatives they intend to adopt within a three years period.

The different sections above are quite broad due to the fact that the questionnaire was a joint construction as mentioned previously. Therefore the scope of the questionnaire does not fully correspond to the scope of this thesis and not all of the results from the questions in the questionnaire are analysed here. Instead, the results from the questionnaire survey are used in order to show similarities or dissimilarities with the results from the case study.

\subsubsection{Sample profile and data collection}

The population of this survey study involves LSPs operating on the Swedish market. Since it is not possible to reach the whole population on the Swedish market, a subset of the population, a sample, was targeted. No specific type of LSP companies was targeted (e.g. size or type of services). Forza (2002), stresses that to be able to generalise the answers of the total population the sampling process and the selection of a representative sample play a significant role. In order to get access to contact details for groups of LSPs, information from different types of interest organisation was used in this sampling process. Therefore, the targeted sample of respondents consisted of members in the interest organisations, Sveriges Åkeriföretag and Sveriges Transportindustriförbund.

A mail questionnaire was sent to 636 respondents, but since some companies were not eligible for the study, this figure decreased to 590 relevant respondents. Those respondents that were not eligible included companies where the address was wrong (returned), and these respondents were therefore excluded as not relevant. The survey was launched in early June, 2011 and the response period extended to end of September 2011. During this period, four reminders were sent out to the respondents in order to increase the response rate, and by the end of the period, 74 respondents had filled in the questionnaire (a response rate of 12,5\%).

Regarding the response rate, as high response rate as possible is of course desirable, but usually rates are in the neighbourhood of 20\% (Lambert and Harrington, 1990) and 
studies have been published in operations management journals with response rates ranging from $10 \%$ to $20 \%$ (Flynn et al., 1990). In this thesis, a response rate of $12,5 \%$ with 590 useable answers can be considered as relatively good and enough for the purpose of this thesis. However, the amount of missing data is relatively large, but during the response period, some respondents declined to participate in the study due to the fact that the content of the questionnaire did not reflect the company's activities, and some respondents even claimed that limited time prevented them from answering the questionnaire.

\subsection{ANALYSIS UNIT AND ANALYSIS OF THE RESEARCH QUESTIONS}

One of the significant components of a research design is the unit of analysis (Yin, 2009), which comprises the main entities analysed in a research study. Furthermore, Dubé and Paré (2003) stress the importance for researchers to specify the unit of analysis in order to make it easier for the reader to understand how the case study relates to a broader body of knowledge. The unit of analysis of this licentiate thesis is twofold and include the adoption of green initiatives as well as the effect this can have on the service offerings. Hence, the unit of analysis can be a description of how one single company has undertaken the adoption of green initiatives and the effects of this adoption of the service offering, or it can include descriptions of multiple companies. In this study, as mentioned in section 3.4, focus has been on six different LSPs and their adoption of green initiatives. However, it is important for researchers to decide whether the individual, company, division or corporate level should be selected, as the unit of analysis depends upon the research questions (Flynn et al., 1990).

Before continuing the description of the analysis of the research questions, let us quickly recap what these questions are. As described in Chapter 1, the four research questions of interest for this thesis are:

RQ1: How can different triggers initiate the adoption of green initiatives?

RQ2: How can different drivers and barriers affect the adoption of green initiatives?

RQ3: How can firm characteristics affect the adoption of green initiatives?

RQ4: In which ways can the adoption of green initiatives be reflected in the service offering?

Now, when the research questions and the units of analysis have been presented, the link between the four appended papers and the research questions can be clarified, as can be seen in Table 2 below. All the research questions relate to two or more Papers and in the cases when the content of the Papers is not directly related to the different research questions but still gives some enriching input, this has been illustrated with an (X). Furthermore, even if two of the four Papers are based on the case study, additional input from the case report to the research questions has been added in order to further strengthen or fill out the areas, which have not been included or emphasised in the Papers. 
Table 2 The link between the research questions and the appended Papers.

\begin{tabular}{c|ccccc} 
& Paper 1 & Paper 2 & Paper 3 & Paper 4 & Case Report \\
\hline RQ 1 & $\mathrm{X}$ & $\mathrm{X}$ & & & $(\mathrm{X})$ \\
RQ 2 & & $\mathrm{X}$ & & $(\mathrm{X})$ & $\mathrm{X}$ \\
RQ 3 & & $\mathrm{X}$ & & $(\mathrm{X})$ & $(\mathrm{X})$ \\
RQ 4 & $\mathrm{X})$ & & $\mathrm{X}$ & & $(\mathrm{X})$
\end{tabular}

The analysis in this thesis frame has been structured around and divided into the four research questions presented. Moreover, the analysis for each of the research questions is based on those Papers that can contribute to finding an answer to that particular research question, as illustrated above. As described in Chapter 1, the focus of the first three research questions is mainly the adoption of green initiatives by LSPs, while the fourth and last research question concerns in which ways the adoption of green initiatives can be reflected in the service offering, see Figure 5 below.

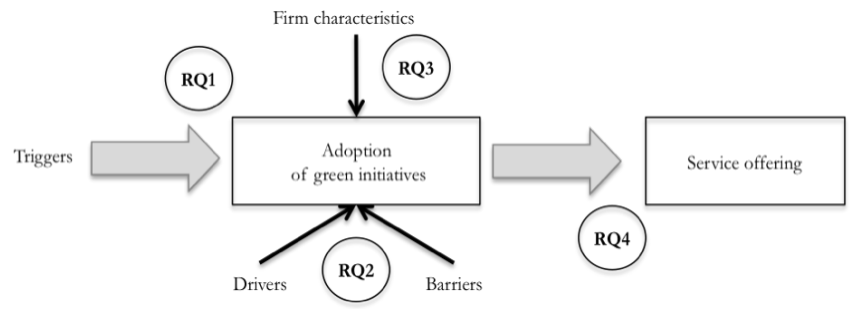

Figure 5 The main objective for each of the Research Questions.

The analysis has been carried out in a chronological order and therefore starts with Research Question 1. The first step of the analysis aims therefore to find out how different triggers can affect LSPs adopting of green initiatives, which in turn also can provide an understanding of why they choose to start adoption green initiatives. Input for this analysis comes first and foremost from Paper 1 and Paper 2, but additional input has also been provided by the case report. Paper 1 identifies six different main aspects that may influence a LSP to start adopting green initiatives. These aspects are solely derived from the literature. Paper 2, on the other hand, is empirically based and identifies some of the triggers for LSPs to start to adopt green initiatives. Note, however, that triggers here, are equated with the main incentives mentioned in Paper 2. The analysis aims to compare the literature findings from Paper 1 and the empirical results from Paper 2 in order to analyse how different triggers can initiate the adoption of green initiatives undertaken by LSPs.

Research Question 2, which can be seen as the second step of the analysis, aims to provide how different drivers and barriers can affect the adoption of green initiates. Different drivers and barriers that may influence the adoption of green initiatives have been found in the literature, and the main empirical input to this question is provided by the case report to six different LSPs. The analysis is divided into two parts; namely drivers and barriers. Tables were developed to summarise the impacts of these drivers and 
barriers in order to enable comparison across the data. This approach is recommended by Yin (2009) who also states that this can facilitate the highlighting of possible patterns that could emerge from findings. However, the main focus of the analysis has been to find out how these drivers and barriers affect the adoption of green initiatives among the studied LSPs. The results of the analysis of the case study have been compared with the results from Paper 2. Furthermore, the results from Paper 4 have also been compared with the result of the analysis, and similarities and differences have been emphasised.

Note, however, that the results of the survey have been updated and include more responses than the results presented in Paper 4. In addition, the drivers and barriers studied in the survey are basically those that are found to be relevant in the case study. However, a few minor changes have been made in the way that these have been formulated and the survey has also been expanded with some additional drivers and barriers.

The aim of the third research question is to investigate how firm characteristics can affect the adoption of green initiatives. The main input to this question is Paper 2, which present some differences between small and large LSPs when these adopt green initiatives. Moreover, the results obtained from Research Question 2 enabled further identification of how firm characteristics can affect the adoption of green initiatives. Furthermore, additional input and examples has also been provided from Paper 4.

As mentioned earlier, Research Questions 1-3 focus on the adoption of green initiatives, while the aim of Research Question 4 is to find out in which ways the adoption of green initiatives can be reflected in the service offering. The analysis of this research question may be considered to be of a more descriptive character due to the fact that the selected LSPs are in an early phase of their adoption of green initiatives and thus have no elaborated approach for how this should be reflected in their service offerings. The main input to this analysis is provided by Paper 3. The analysis process of Paper 3 can be illustrated by the three different steps of data analysis presented by Miles and Huberman (1994) namely: data reduction, data displays and conclusions drawing. Data reduction refers to the process of sharpening and organizing the data, while data displays allow the researcher to concentrate on a reduced set of data, which in turn, helps to show patterns. The last step calls for further analysis in the data displays, which in turn facilitates the seeing of further conclusions. The results from Paper 3 are compared with the literature findings regarding the green service offerings presented in Paper 1.

\subsection{THE RESEARCHER'S OWN RESEARCH PROCESS}

This subsection aims to give the reader a view of my own research process, including the path and the decisions taken which led to my research area, the research project to which I belong and my contributions to the papers appended to this thesis.

\subsubsection{The path to the research area}

I started as a PhD student for almost two and a half years ago. These years have been full of lessons and experiences, both in terms of my own research process and also what it actually is to be a PhD student. I have realized that working as a doctoral student should rather be classified as a lifestyle than a "normal" job. It was during my time as a student 
on the MSc Programme, Industrial Engineering and Management at Linköping University, that my keen interest in becoming a PhD student arose. My economics specialisation was within supply chain management and industrial marketing, so the fact that my research would be within the logistics area was already a natural choice when I was a student. However, my interest in marketing got me started to think whether it was possible to combine these two areas and integrate them within my own research.

In a relatively early phase of this process, I started to take an interest in the service offerings provided by LSPs and identified it as the missing link to combine logistics and marketing in my own studies. Today's transport system is largely based on responding to market demands, where customers' desires for more diverse services are becoming more common. However, the demand for service offerings to fulfil environmental requirements does not seem to increase to the same extent. This may due to a variety of reasons, such as lack of interest, unwillingness to pay extra for these kinds of service offerings and also perhaps a lack of knowledge of what is it possible to do. Therefore, my growing interest in considering environmental issues in the development of logistics services led to a conference paper. This paper (which is called Paper 1 in this thesis) was based on literature reviews and compared sustainable service and product development research with literature about development of offerings provided by LSPs. The findings of the paper became a framework based on different main aspects, which either directly or indirectly could affect the service offering from a green perspective. Hence, in order to get a deeper understanding how these aspects could affect LSPs' efforts to greening their business as well as their service offerings, led to Paper 2. Since the awareness and knowledge of environmental impact is increasing among society, this trend is also reflected to some extent in today's businesses. In order to investigate the current situation within the LSP industry, a case study including six LSP companies active on the Swedish market was conducted. Some outcomes of this case study, together with a similar study conducted in Italy and Spain resulted in Paper 2. This paper focuses on green awareness among LSPs as well as the identification of different kinds of drivers and barriers affecting the adoption of green initiatives. Paper 2 is concerned with the green development within LSPs on an overall company level and does not reflect the effect of the service offering. Therefore, in order to take a step further, Paper 3 was written. This partly aimed to seek the underlying rationale behind the development of green service offerings among LSPs. In contrast to Paper 2, this paper was solely based upon the Swedish case study.

In order to broaden the scope and also to validate the findings from the case studies, a decision was taken to conduct a questionnaire survey. Originally, this questionnaire survey was only intended for the Swedish market, but after interest was expressed by other international researchers, (as mentioned in section 3.5), the questionnaire survey has been and is planned to be launched in other European countries as well. The preliminary findings from the questionnaire survey (mainly based on the Swedish results but also on a few Italian respondents) resulted in a conference paper, presented as Paper 4 in this licentiate thesis. This paper concerns different drivers and barriers affecting the greening of LSPs' activities, and also raises the question whether firm characteristics may affect an LSP's adoption of green initiatives. Not only does Paper 4 to some extent, validate the prior findings from the case studies but it also provides a basis for further research. 
Finally, writing this section about my own research process, which for the reader is perhaps perceived to be quite straightforward and chronologic, reminded me that this process was not always without complexity. For example, early in the process I focused my research on different price models which took green aspects into account, but since LSPs had not reached this stage in their green development and perhaps never will, this focus received less attention in my research.

\subsubsection{The research project}

The research presented in this dissertation, is partly the result of a three years long research project in which I was involved for two and a half years in the department of Logistics Management, at Linkoping University. The research project, funded by the Swedish Governmental Agency for Innovation Systems, VINNOVA, is titled Competitive business models to meet future demands on sustainable logistics systems - a research project aiming at more proactive logistics firms, and involved collaboration with Swedish actors on the logistics market as well as other international universities.

The primary aim of the research project was to increase the knowledge of how the impact on the environment by transportation of goods may be affected by a wider understanding of the logistics system boundaries, and also by a new way to manage as well as new roles in the logistics system. The research project focused initial by three main areas namely:

- Future business models and new roles for proactive logistics companies

- Evaluation of the environmental aspects of the company's logistics system from a business perspective

- Control and monitoring of the logistics system's environmental performance

My research mainly focuses on the first part of the research project but also tends to touch on the second part of the research project. The main objective of the first part is to examine how an expanded logistics system, which includes activities, processes, decisions and responsibility mainly carried out by shippers today, could give better conditions for creating more effective systems. These changes in form of what can be changed and who will be responsible to perform these changes may increase the potential for logistics companies to develop new services and also take new roles in the logistics system. Even though the research project involves efforts from several researchers both from my own research group and other international universities, this thesis is based on the my own research and the contributions from other researchers are acknowledged below.

\subsubsection{The researcher's contribution to the Papers}

All the appended papers in this licentiate thesis have been written together with at least one senior researcher. In general, even if the authors responsible were equally familiar with the content of the paper, the contribution of the different authors varied for each of the papers. Therefore, the following text outlines my main contributions for each of the papers appended here.

The initiative of Paper 1 came from me and the paper was conducted together with my supervisor and co-author Maria Björklund. The overall outline of the Paper was jointly discussed although I was mainly responsible for this process. In addition, I was 
responsible for the first parts of the paper including the introduction, methodology and theoretical framework with some guidance and support from my supervisor. The content of the analysis and the discussion were a joint product even if Maria Björklund was responsible for completing it.

As mentioned earlier in section 3.7.1, Paper 2 is based on two conferences proceedings and was a joint initiative between the responsible authors, myself, my supervisor Maria Huge-Brodin, Pietro Evangelista and Edward Sweeney. My main contributions are half the theoretical framework and half of the empirical basis including all the Swedish case companies. However, I have more or less contributed to all the different sections of the paper since I was responsible for combining the two conference proceedings into one paper including first and foremost an introduction, the research design, theoretical framework and empirical basis. Furthermore, I also contributed to a first draft of the analysis and made suggestions for the discussion part of the paper.

As in Paper 2, my involvement in Paper 3 relates to all parts of the paper. I was responsible for writing a draft of the first parts of the paper up to the analysis section. The outline and the main part of the analysis work were then done in discussion with my co-author Maria Huge-Brodin. The analysis section was divided into two parts where I was responsible for compiling the first part. Like the analysis, the conclusions were done in collaboration between Maria Huge-Brodin and myself.

Paper 4, beside myself involved the following authors, Maria Björklund, Maria HugeBrodin and Pietro Evangelista. The paper is based on a questionnaire survey for which I had the main responsibility. I also wrote the introduction and methodology section and provided input to the analysis as well as to the conclusions even though it was Maria Björklund who did the analysis work. 


\section{Summary of The APPENDEd Papers}

This chapter presents a summary of each of the four appended papers in this licentiate thesis. The summary presents the aim of the study and the focus is on the main results and contributions of the paper. For further information and the papers in their entirety can be found in Appendixes 4, 5, 6 and 7.

\subsection{Paper 1 Developing Sustainable Logistics Services}

The increasing awareness of environmental impacts on our society among the public, is resulting in increased demands on governments and industries to actually respond and act to these issues. This is turn can lead to higher demands for "green" products, more stringent environmental regulation by the governments, and also a more environmentally responsible business management (Facanha and Hovath, 2005; Srivastava, 2007). This is of specific interest to logistics companies, whose core business is often an environmental impact in itself. Due to the increased demands from society as well as to strengthen their position on the market, logistics companies need to take into account of environmental aspects into their services. Therefore, in order to acquire a comprehensive picture of what this can involve, the purpose of this paper is as followed;

To present a framework for how to consider sustainability in the development of logistics services. In addition, the paper also aims at developing a research agenda for further research needs regarding how logistics companies can include the environmental aspect in their service offerings.

The paper was based on a literature review that had its origin in sustainable service and product development literature. This was compared with literature about the development of offerings for logistics companies. The results from the literature studies were then analyzed in order to identify a research agenda and to present a framework that included aspects of sustainable service offerings of relevance from a logistics company perspective.

From the first literature review in the paper, six main aspects affecting the development of sustainable services and products were identified, namely: customer and market, collaboration (internal/external), business management and strategy, ICT, means of control and competence and knowledge. Furthermore, the extent of their relevance in the development of environmentally sustainable services for logistics companies is considered in the framework developed, see Table 3 below. The framework aims to show why these aspects should be considered, how the aspects affect the development of the offering, and how these aspects can be visualized in the offering. 
Table 3 Framework of environmentally sustainable services for logistics companies.

\begin{tabular}{|c|c|c|c|}
\hline Aspects & Benefits (Why involve) & $\begin{array}{l}\text { Considerations in the } \\
\text { development of the offering }\end{array}$ & The offering \\
\hline \multirow{3}{*}{ 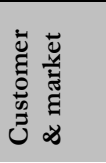 } & Identify attractive services & $\begin{array}{l}\text { Customer demands as starting } \\
\text { point }\end{array}$ & $\begin{array}{l}\text { Diversified \& customised } \\
\text { offers }\end{array}$ \\
\hline & Focus on the "right" aspects & Early integration of marketing & $\begin{array}{l}\text { Pricing the green service } \\
\text { offering }\end{array}$ \\
\hline & Develop wanted services & & Customer segmentation \\
\hline \multirow{7}{*}{ 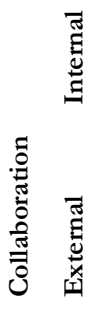 } & Knowledge exchange \& spread & Use of cross functional teams & \\
\hline & Efficient problem solving & Include environmental experts & \\
\hline & & Identify collaborations risks & \\
\hline & $\begin{array}{l}\text { Improved supplier } \\
\text { development }\end{array}$ & Develop methods to handle risks & Show awareness \\
\hline & Shared data \& knowledge & Collaborate with suppliers & Show willingness to collaborate \\
\hline & $\begin{array}{l}\text { Improved sustainability } \\
\text { performance }\end{array}$ & Collaborate with customers & \\
\hline & & Introduce the collaboration early & \\
\hline \multirow{5}{*}{ 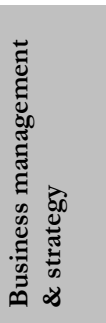 } & $\begin{array}{l}\text { Cascade sustainability } \\
\text { throughout the organisation }\end{array}$ & $\begin{array}{l}\text { Integrate strategic, tactical \& } \\
\text { operational level cross the } \\
\text { company }\end{array}$ & $\begin{array}{l}\text { Hard to visualise in the } \\
\text { offering but might be done } \\
\text { with the use of certain } \\
\text { certifications and standards }\end{array}$ \\
\hline & $\begin{array}{l}\text { Increase the environmental } \\
\text { benefits }\end{array}$ & Support from top management & \\
\hline & $\begin{array}{l}\text { "Common language" among } \\
\text { employees }\end{array}$ & & \\
\hline & Achieve corporate commitment & & \\
\hline & Increased trustworthiness & & \\
\hline \multirow[b]{4}{*}{ 包 } & Enable customisation & Internal measuring & Provide customised offerings \\
\hline & Meet customer demands & $\begin{array}{l}\text { Collect and store environmental } \\
\text { data }\end{array}$ & $\begin{array}{l}\text { Display/measure } \\
\text { environmental impact }\end{array}$ \\
\hline & $\begin{array}{l}\text { Control of environmental } \\
\text { impact }\end{array}$ & & Facilitate collaboration \\
\hline & $\begin{array}{l}\text { Support development of new } \\
\text { offerings }\end{array}$ & & \\
\hline \multirow{2}{*}{ 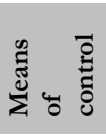 } & Stimulate development & Focus on outcome not method & $\begin{array}{l}\text { Use labelling/standards in the } \\
\text { offering }\end{array}$ \\
\hline & Economical advantages & Focus/select e.g. standards & $\begin{array}{l}\text { Trustworthy \& informative } \\
\text { offerings }\end{array}$ \\
\hline \multirow{4}{*}{ 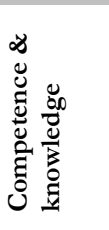 } & Strengthen skills \& knowledge & Training \& development & $\begin{array}{l}\text { Knowledge as part of the } \\
\text { offering }\end{array}$ \\
\hline & Facilitate increased innovation & $\begin{array}{l}\text { Adjust knowledge needs to strategy } \\
\text { and ambitions }\end{array}$ & Trustworthy offers \\
\hline & Maintain \& keep competence & $\begin{array}{l}\text { Identify potential knowledge } \\
\text { exchange with extern }\end{array}$ & \\
\hline & Improved performance & & \\
\hline
\end{tabular}

The applicability and generalization of the presented framework can be seen as a source of inspiration for both researchers and logistics companies. The findings should be considered as conceptual, and the paper also presents a research agenda for the main aspects identified in the framework and consequently provides a basis for further empirically based research. 


\subsection{PAPer 2 Green Initiatives in the Transport and Logistics SERVICE INDUSTRY: AN EXPLORATORY CASE STUDY OF Logistics Service Providers}

The impact of business operations on the natural environment has become one of the main areas that society and governments have paid more attention into. This in turn has led to a growing pressure on managers to react and deal with green issues, not only within their own firms, but also for their supply chain partners (Vachon and Klassen, 2006). Environmental research specific to LSPs have been more or less neglected, despite the fact that LSPs have assumed a more critical role in supply chain orchestration and management. Therefore, in order to generate more knowledge and understanding of the green initiatives undertaken by LSPs, the purpose of this paper is to:

Explore and describe the awareness and adoption of green initiatives among LSPs, as well as identify drivers and barriers affecting green initiatives undertaken by LSPs.

This has been done through a multiple case study approach, which investigates eight cases of LSPs operating in Sweden, Italy and Ireland (four Swedish, two Italian and two Irish). The analysis undertaken in this article was divided into the following objectives:

- analyse awareness of the importance of sustainability in LSPs companies;

- analyse the adoption of green initiatives by LSPs;

- identify drivers affecting the adoption of green initiatives by LSPs; and,

- identify barriers to the adoption of green initiatives by LSPs.

Furthermore, the case companies were divided into two categories; large and small firms, in order to enable the identification of the influence that firm size may have on the adoption of green initiatives. Based on the analysis and the findings of the cases, a set of propositions was derived from each of the four objectives.

\section{Awareness of green initiatives}

Awareness of the importance of green issues is well demonstrated among the case companies. The case companies gave a unanimous picture of why LSPs consider adopting green initiatives in the first place, and stated that customers and brand image were their two main incentives. Another interest finding concerned the role of top management as a main incentive. The LSPs that identified top management as a main incentive for adopting green initiatives also had a stronger focus on more long-term strategic priorities. Two propositions were derived from the findings and analysis of this objective:

- Proposition A: As top management embraces green initiatives, the long-term strategic priority accorded to green initiatives increases.

- Proposition B: As employees initiate green improvements, the short-term priority accorded to green initiatives increases. 


\section{Adoption of green initiatives}

A common picture among the studied cases is that many green initiatives directly involve transportation in a variety of ways. In relation to ICT solutions, it emerges that LSPs do not widely use technology to support green initiatives. Furthermore, the level of ICT adoption is still low, especially in small companies. Two propositions was suggested in order to try explain the differences in the adoption of ICT to support green initiatives:

- Proposition C: The larger the LSP, the higher the tendency to use more sophisticated ICT tools to support green initiatives.

- Proposition D: The wider the service offering, the higher the tendency to use more sophisticated ICT tools to support green initiatives.

\section{Drivers affecting the adoption of green initiatives}

The prominent drivers of green initiatives among the case companies were the managerial driver and the employees, and discussion concerning the impact of these drivers on adoption of green initiatives resulted into two propositions:

- Proposition E: As employee engagement increases, the adoption of green initiatives increases.

- Proposition F: As managerial support for green initiatives increases, the resistance from employees decreases.

Competitors were not seen as a major driving force among the case companies. However, since competitors are often mentioned as a driver in the literature and might therefore advance the development of green initiatives further, a more future-oriented proposition was formulated:

- Proposition G: As pressure from competitors increases, the adoption of green initiatives increases.

Environmental legislations and regulations were also identified as significant drivers among the case companies. Another significant driver identified in the Paper were customers (at least among the Swedish case companies). Based on additional findings and analysis, this generated into two propositions:

- Proposition H: As green legislation and regulation increases, customer engagement increases.

- Proposition I: As green information from LSPs increases, customer engagement increases.

\section{Barriers affecting the adoption of green initiatives}

Lack of customer/market support, as well as financial barriers, proved to have a significant impact in the investigations. Other barriers mentioned were for example, lack of information and competences in the area of green logistics, and also a certain resistance within the actual organisation regarding green initiatives. This led to the following propositions: 
- Proposition J: As collaboration with customers increases, long-term commitment and the willingness to share costs increase, and financial barriers to green initiatives decrease.

- Proposition K: As the level of ICT adoption increases, the knowledge level of green issues among employees and customers increases.

\subsection{Paper 3 Understanding EfFiciencies Behind Logistics Service Providers' Green Offerings}

Due to the increasing importance of green management over the past decade, LSPs need to make more efforts in green issues in order to deliver products and services in more environmentally friendly ways. The ability of green management to integrate green issues into logistics service offerings has involved more and more LSPs and will probably attract even more managerial attention in the logistics industry in the future (Lieb and Lieb, 2010). Hence, this will be a challenge for LSPs, and it is clear that there is a lack of both knowledge and research in this area. Therefore, the article takes an LSP perspective, with the purpose of:

Indicating where green-labelled LSPs are positioned today in their development of green service offerings and furthermore to explain the underlying rationales behind the development of green service offerings.

This article is based on a multiple case study of 6 LSPs, active on the Swedish market and the goal was to build an explanation about the cases and develop ideas for further study. The analysis was based on 6 new service development dimensions derived from the literature, and the analysis process was divided into two steps:

- an analysis of the service dimensions per se, describing green service offerings and identifying factors affecting these among the case companies: and

- cross-case analysis of each of the six cases, resulting in the identification of patterns across the service dimensions and suggesting explanations for these patterns.

As mentioned above, the first step analyses the six dimensions of new service offering development, and relates the case findings mainly to this six-dimensional model. The results of the first analysis are summarised in Table 4, below. 
Table 4 Summary of the most evident case characteristics identified according to the 6 different dimensions of new service offering development.

\begin{tabular}{|c|c|c|c|c|c|c|}
\hline \multirow{2}{*}{$\begin{array}{l}\text { Service } \\
\text { dimensions }\end{array}$} & \multicolumn{6}{|c|}{ Case companies } \\
\hline & Alfa & Beta & Gamma & Delta & Epsilon & Zeta \\
\hline $\begin{array}{l}\text { New service } \\
\text { concept or offering }\end{array}$ & $\begin{array}{l}\text { Green } \\
\text { choice }\end{array}$ & $\begin{array}{l}\text { Integration/ } \\
\text { green choice }\end{array}$ & $\begin{array}{l}\text { Full } \\
\text { integration }\end{array}$ & $\begin{array}{l}\text { Full } \\
\text { integration }\end{array}$ & $\begin{array}{l}\text { Integration/ } \\
\text { green choice }\end{array}$ & Integration \\
\hline $\begin{array}{l}\text { New customer } \\
\text { interaction }\end{array}$ & $\begin{array}{l}\text { Wide range } \\
\text { of customers }\end{array}$ & $\begin{array}{l}\text { Focused } \\
\text { customer } \\
\text { segments }\end{array}$ & $\begin{array}{l}\text { Wide range } \\
\text { of customers }\end{array}$ & $\begin{array}{l}\text { Focused } \\
\text { segments }\end{array}$ & $\begin{array}{l}\text { Wide range } \\
\text { of customers }\end{array}$ & $\begin{array}{l}\text { Focused } \\
\text { segments }\end{array}$ \\
\hline $\begin{array}{l}\text { New value } \\
\text { system/business } \\
\text { partners }\end{array}$ & $\begin{array}{l}\text { Networking } \\
\text { with } \\
\text { competitors }\end{array}$ & $\begin{array}{l}\text { Networking } \\
\text { with } \\
\text { competitors } \\
\text { and } \\
\text { authorities }\end{array}$ & $\begin{array}{l}\text { Competitors } \\
\text { awareness }\end{array}$ & $\begin{array}{l}\text { Competitors } \\
\text { awareness }\end{array}$ & $\begin{array}{l}\text { Networking } \\
\text { with } \\
\text { research and } \\
\text { in general }\end{array}$ & $\begin{array}{l}\text { Competitor } \\
\text { awareness }\end{array}$ \\
\hline $\begin{array}{l}\text { New revenue } \\
\text { model/pricing }\end{array}$ & $\begin{array}{l}\text { New market } \\
\text { shares. No } \\
\text { price models }\end{array}$ & $\begin{array}{l}\text { New market } \\
\text { share/added } \\
\text { value. Price } \\
\text { raise for } \\
\text { non- } \\
\text { partners. }\end{array}$ & $\begin{array}{l}\text { New market } \\
\text { share. No } \\
\text { price models } \\
\text { (strategic } \\
\text { decision) }\end{array}$ & $\begin{array}{l}\text { New market } \\
\text { shares. No } \\
\text { price model } \\
\text { (strategic } \\
\text { decision) }\end{array}$ & $\begin{array}{l}\text { Competitive } \\
\text { pricing to } \\
\text { cover green } \\
\text { investments }\end{array}$ & $\begin{array}{l}\text { Competitive } \\
\text { pricing. No } \\
\text { price models } \\
\text { (strategic } \\
\text { decision) }\end{array}$ \\
\hline $\begin{array}{l}\text { New service } \\
\text { delivery system; } \\
\text { organisation, } \\
\text { personnel, culture }\end{array}$ & $\begin{array}{l}\text { Top-down. } \\
\text { Specialised }\end{array}$ & $\begin{array}{l}\text { Bottom-up. } \\
\text { Broad } \\
\text { engagement }\end{array}$ & $\begin{array}{l}\text { Bottom-up. } \\
\text { Specialised }\end{array}$ & $\begin{array}{l}\text { Top-down. } \\
\text { Broad } \\
\text { engagement }\end{array}$ & $\begin{array}{l}\text { Top-down }+ \\
\text { bottom-up. } \\
\text { Specialised }\end{array}$ & $\begin{array}{l}\text { Top-down- } \\
\text { Specialised }\end{array}$ \\
\hline $\begin{array}{l}\text { New service } \\
\text { delivery system; } \\
\text { technology }\end{array}$ & Await & $\begin{array}{l}\text { Fast } \\
\text { development }\end{array}$ & $\begin{array}{l}\text { Slow } \\
\text { development }\end{array}$ & Await & $\begin{array}{l}\text { Fast } \\
\text { development }\end{array}$ & Await \\
\hline
\end{tabular}

The second step of the analysis presents the patterns, which were identified when the different dimensions were combined within the cases, and explanations for the empirically generated patterns are sought in literature on efficiency in organisations. However, due the fact that LSPs could be seen in an early phase of the green development process, three different patterns that reflected the current behaviour and anticipations among the case companies could be discerned. The different patterns and their overall focus are;

- Focus and abide versus network and explore - focuses on the strategic standpoint of the greening of LSPs activities and the competitive awareness of the development in the logistics market.

- Internal focus for general "spread" versus broad engagement to target specific customers - involves customer orientation and transferring and sharing green knowledge and competences.

- Top-controlled passiveness versus bottom-up exploration of technology concerns how green activities are managed and controlled within the company and the investment of resources.

Lin and Ho (2008) stress that research needs to determine the potential factors that will influence the willingness to adopt green innovations for service sectors. In general, the analysis in this article gives a broad picture in which settings, and the strategies adopted to address green development for the company and service offerings differ slightly among the case companies. The study presented offers some tentative explanations, but to 
enhance a deeper understanding, future research needs to focus on barriers and drivers as well as on how different stakeholders influence the greening of LSPs.

\subsection{Paper 4 The Challenge and Adoption of Green Initiatives For Transport and Logistics Service Providers}

The level of initiatives needed to develop and introduce new products and services to provide shippers with green transport and logistics options differs among LSPs. For that reason, this study aimed to increase the knowledge of how LSPs face the challenge of greening their operations according to their own business and its context characteristics. Therefore, an important point of departure is to understand the underlying mechanisms in the form of drivers and barriers, as well as the firm characteristics that affect companies in their work to green their operations. The purpose of the paper is as follows:

To develop a basis for further investigations of green initiatives carried out by LSPs and analyse whether the green initiatives implemented are dependent on firm characteristics of the LSPs, as well as on the drivers and barriers experienced.

This paper is based on a questionnaire survey and investigates a sample of LSPs operating on the Swedish and Italian markets. Due to a delayed launch of the questionnaire, only a limited number of valid completed questionnaires were collected before the deadline for this paper. Therefore, the Italian and the Swedish answers were treated as one single group. The preliminary analysis aimed to identify the largest drivers/barriers as well as common green initiatives, see figure 6. T-tests were applied in order to identify statistical differences between the means. Furthermore, step-wise regression analyses were performed in order to identify significant relationships between the initiatives implemented (dependent variables) and the barriers and drivers (independent variables).

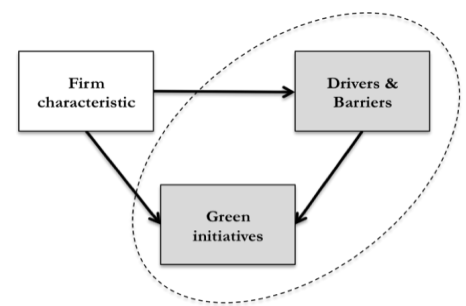

Figure 6 Analysis model

\section{Drivers to greening logistics/initiatives}

The role of different stakeholders' influence in driving LSPs to develop and implement green initiatives was investigated. The results showed that top management and customers were seen as the strongest drivers whereas insurance companies and experts were shown to have a minor role. Furthermore, a variety of different reasons were also identified to support and drive the implementation of green initiatives among LSPs. All the reasons studied perceived a quite similar and high influence. However, the highest means were identified for reasons such as increasing firm's competiveness, improving 
customer relationship, improving brand image as well as customer service. This in turn indicates the importance of the customers in implementing green initiatives among LSPs.

\section{Barriers to greening logistics/initiatives}

The barriers investigated, to implementing green initiatives, divided into internal and external, seemed in general to be less important than the drivers. The external barrier with the highest mean was lack of economic incentives while negative impact on the customer supply chain as well as lack of ICT vendors received the lowest means. Cost for investments and an uncertain payback period were experienced as the largest internal barriers towards the implementation of green initiatives and the lowest mean was identified for external ICT skills.

\section{What influences the green initiatives?}

In order to identify the influence from the strongest contingency factors (barriers and drivers), a regression analysis was conducted. However, no barriers were included in this analysis due to their relatively low influences. The results from the regression analysis indicated that customers do seem to have an important and significant influence on the implementation of green initiatives, see figure 7 below.

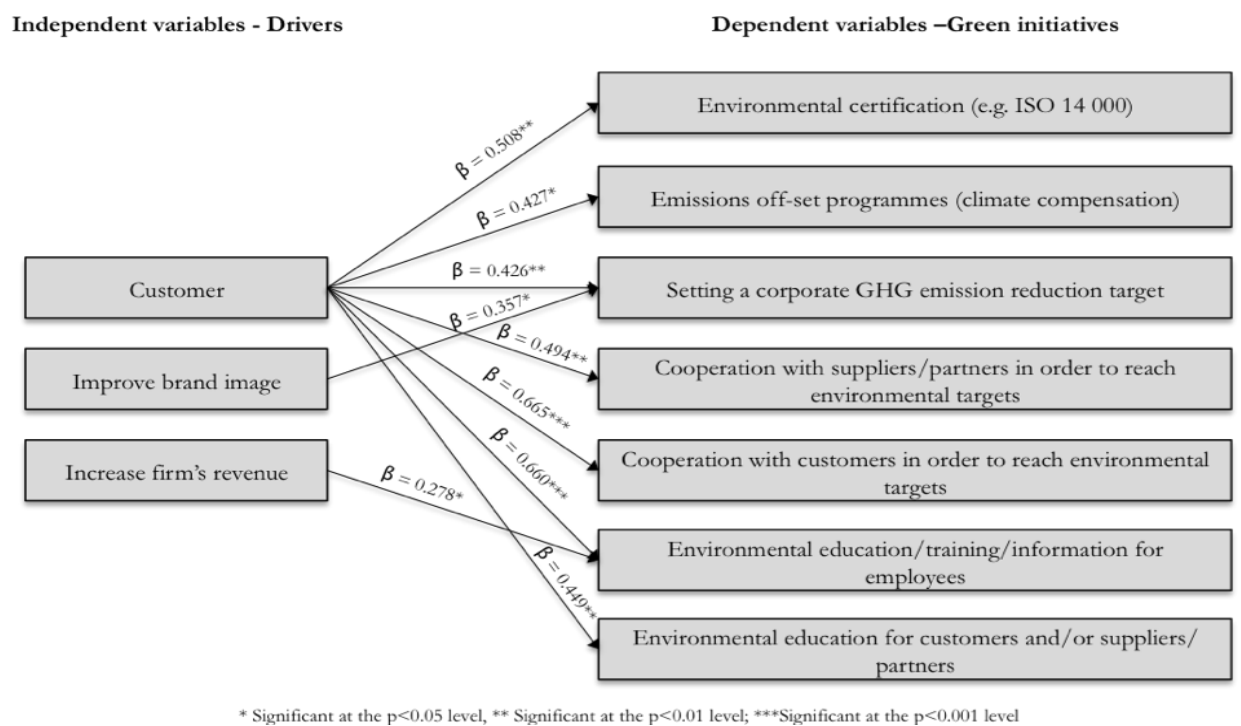

Figure 7 Regression analysis of strong drivers and green initiatives

As mentioned earlier, the paper provides, a basis for further analysis to investigate whether for example different green initiatives are adopted by different LSPs. This can be achieved by classifying a number of firm attributes (e.g. size, geographical distribution, range of service offerings, the level of ICT adoption) and correlating these attributes to the green initiatives adopted and the type of drivers and barriers affecting these companies. Furthermore, a higher response rate than the presented response rate in this paper can also make it possible to carry out a factor analysis in order to group the barriers and drivers experienced, as well as the initiatives implemented. 


\section{ANALYSIS}

The analysis of this Thesis Frame is a joint analysis of the four appended Papers but additional input also comes from the comprehensive report of the case study (see Appendix 2) as well as additional results from the questionnaire survey. The chapter is divided and structured according to the four research questions presented in Chapter 1. The analysis of the each of the research questions starts with its position in the research process as well as its main input, and ends with a concluding remark.

\subsection{HOW CAN DIFFERENT TRIGGERS INITIATE THE ADOPTION OF GREEN INITIATIVES?}

The first research question considers how different triggers can initiate the adoption of green initiatives. The position of this research question in the analysis and its main input is illustrated in Figure 8, below.

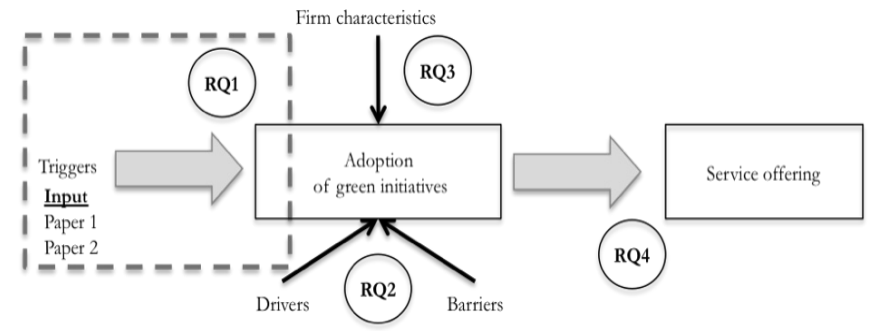

Figure 8 The position of Research Question 1.

As can be seen in the figure, the main input for Research Question 1 is based on results from Paper 1 and Paper 2, although the papers have slightly different approaches to concern this specific area.

As mentioned earlier in Chapter 3 and Chapter 4, Paper 1 is based on literature reviews, which combine the two areas: development of green service offerings and development of logistics service offerings. By combing the experience from these two fields, six different main aspects were identified, as well as the benefits of and insights into how these main aspects can be considered in the development of green service offerings for LSPs, see Table 5. 
Table 5 The identified framework in Paper 1.

\begin{tabular}{|c|c|c|c|}
\hline Aspects & Benefits (Why involve) & $\begin{array}{l}\text { Considerations in the } \\
\text { development of the offering }\end{array}$ & The offering \\
\hline \multirow{3}{*}{ 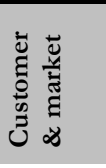 } & Identify attractive services & $\begin{array}{l}\text { Customer demands as starting } \\
\text { point }\end{array}$ & $\begin{array}{l}\text { Diversified \& customised } \\
\text { offers }\end{array}$ \\
\hline & Focus on the "right" aspects & Early integration of marketing & $\begin{array}{l}\text { Pricing the green service } \\
\text { offering }\end{array}$ \\
\hline & Develop wanted services & & Customer segmentation \\
\hline \multirow{7}{*}{ 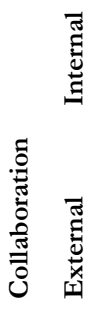 } & Knowledge exchange \& spread & Use of cross functional teams & \\
\hline & Efficient problem solving & Include environmental experts & \\
\hline & & Identify collaborations risks & \\
\hline & $\begin{array}{l}\text { Improved supplier } \\
\text { development }\end{array}$ & Develop methods to handle risks & Show awareness \\
\hline & Shared data \& knowledge & Collaborate with suppliers & Show willingness to collaborate \\
\hline & $\begin{array}{l}\text { Improved sustainability } \\
\text { performance }\end{array}$ & Collaborate with customers & \\
\hline & & Introduce the collaboration early & \\
\hline \multirow{5}{*}{ 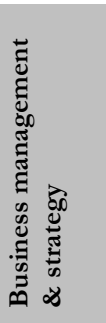 } & $\begin{array}{l}\text { Cascade sustainability } \\
\text { throughout the organisation }\end{array}$ & $\begin{array}{l}\text { Integrate strategic, tactical \& } \\
\text { operational level cross the } \\
\text { company }\end{array}$ & $\begin{array}{l}\text { Hard to visualise in the } \\
\text { offering but might be done } \\
\text { with the use of certain } \\
\text { certifications and standards }\end{array}$ \\
\hline & $\begin{array}{l}\text { Increase the environmental } \\
\text { benefits }\end{array}$ & Support from top management & \\
\hline & $\begin{array}{l}\text { "Common language" among } \\
\text { employees }\end{array}$ & & \\
\hline & Achieve corporate commitment & & \\
\hline & Increased trustworthiness & & \\
\hline \multirow[b]{4}{*}{ 苞 } & Enable customisation & Internal measuring & Provide customised offerings \\
\hline & Meet customer demands & $\begin{array}{l}\text { Collect and store environmental } \\
\text { data }\end{array}$ & $\begin{array}{l}\text { Display/measure } \\
\text { environmental impact }\end{array}$ \\
\hline & $\begin{array}{l}\text { Control of environmental } \\
\text { impact }\end{array}$ & & Facilitate collaboration \\
\hline & $\begin{array}{l}\text { Support development of new } \\
\text { offerings }\end{array}$ & & \\
\hline \multirow{2}{*}{ 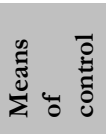 } & Stimulate development & Focus on outcome not method & $\begin{array}{l}\text { Use labelling/standards in the } \\
\text { offering }\end{array}$ \\
\hline & Economical advantages & Focus/select e.g. standards & $\begin{array}{l}\text { Trustworthy \& informative } \\
\text { offerings }\end{array}$ \\
\hline \multirow{4}{*}{ 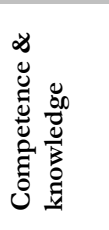 } & Strengthen skills \& knowledge & Training \& development & $\begin{array}{l}\text { Knowledge as part of the } \\
\text { offering }\end{array}$ \\
\hline & Facilitate increased innovation & $\begin{array}{l}\text { Adjust knowledge needs to strategy } \\
\text { and ambitions }\end{array}$ & Trustworthy offers \\
\hline & Maintain \& keep competence & $\begin{array}{l}\text { Identify potential knowledge } \\
\text { exchange with extern }\end{array}$ & \\
\hline & Improved performance & & \\
\hline
\end{tabular}

As illustrated in Table 5, the presented framework consists of the three columns apart from the main aspects identified. The first column, Benefits (Why involve), is of a broader perspective and has therefore a more indirect effect on the green service offerings, while the last two columns, Considerations in the development of the offering and The offering have a clearer and more focused approach on the way in which green considerations could be taken into account and be included in the service offerings. However, since the first research question concerns how different triggers can affect the adoption of green initiatives, the analysis of this section will continue to focus on the main aspects identified and the first column. It is worth mentioning however that green initiatives should not be 
compared with green service offerings in this thesis. Green initiatives are viewed from a broader perspective including issues such as environmental management systems (EMS), environmental certifications (e.g. ISO 14001), emissions off-set programmes, setting a GHG emission reduction target and eco-driving training. Hence, these green initiatives may in turn have an indirect as well as a direct effect on the green service offerings. However, a more detailed and developed analysis of this subject will be addressed later in this chapter in relation to Research Question 4.

As mentioned above, this analysis focuses on the six aspects identified Customer \& market, Collaboration (internal and external), Business management \& strategy, ICT, Means of control and Competence \& knowledge as well as on the benefits generated and the reasons to involve each of these aspects in the business from a green perspective. Therefore, these benefits and reasons identified may in turn be seen as possible triggers for adopting green initiatives. Hence, as pointed out in Paper 1, these six different aspects may perhaps not represent a comprehensive picture, and the findings should be considered as conceptual although the aspects can provide a basis for further empirical based research.

On the other hand, the empirical base and connection to Research Questions 1 can be found from the results in Paper 2, whose theoretical base has been inspired and partly been based on the aspects presented above. One part of this paper aims to analyse the awareness among LSPs today of the importance of green initiatives. The identified triggers, referred to in Paper 2 as the main incentives, that influenced awareness as well as the adoption of green initiatives among the case companies studied can be summarized by:

- Customer

- Brand image

- Competiveness

- Top management

- Cost reductions

The following analysis will focus on these triggers and compare them to the aspects identified in Paper 1 in order to give a closer description of how these triggers might initiate the studied LSPs' adoption of green initiatives, starting with the customers. Customers were frequently mentioned among the case companies studied in Paper 2 as well as in the wider case study (see Appendix 2) as having a significant impact on their increased awareness about sustainability. Not surprisingly, in Paper 1 customers were also found to have a central role, as one of the main aspects Customer and market, and customers' needs and demands were identified as an important starting point for integrating green thinking and adopting green initiatives within a company.

Furthermore, the literature in Paper 1 suggests that in order to improve environmental performance, companies need to identify and understand the functionality required in order to meet the customers' needs and also in order to extend the companies' scope as well as skills regarding this issue (Pujari et al., 2003; Coley and Lemon, 2007; Maxwell et al., 2006). This situation could also be relevant for LSPs, and the results in Paper 2 indicate that there is a need to improve relationships with customers as well as a desire to 
be involved in a more green strategic partnership with them. One of the consequences of being part of a strategic partnership could be that it might lead to more customized solutions. In the green service offering literature referred to Paper 1, the importance of avoiding "one size fits all" when developing green service solutions is highlighted, and customisation and contextualisation are mentioned as critical issues meeting many sets of different needs (Evans et al., 2007). However, as mentioned in Paper 1, the knowledge about the extent to which green service offerings can be customized and the benefits of customizing these are lacking in the literature. Another issue, which it is relevant to consider when entering into a strategic partnership is the choice of customers to collaborate with. The results in Paper 1 stress the importance of segmenting customers, and suggest some guidelines regarding how to segment customers from a green perspective (environmental awareness, ambitions and willingness to pay).

Another trigger, mentioned by the case companies in Paper 2, was brand image. The companies sought to improve their brand image through their engagement with green initiatives. Some of the case companies specifically mentioned a desire to be a "good" company, both for society and for employees, and to take responsibility proactively as a result of the contribution of transport to environmental degradation. Moreover, one of the case companies also highlighted its specific aim to profile the entire company's operations as "logistically sustainable". Brand image was not specifically brought up in Paper 1, but this trigger has nevertheless a clear correlation to the main aspect, Business management and Strategy. As mentioned in Paper 1, integrating green aspects into business management and strategy is not considered to be fully addressed within the logistics literature, even if this is as important for LSPs as for any other service provider. Therefore, a more green profile of the brand image could facilitate the visualization of the integration and adoption of green thinking and initiatives within the company. In addition, a green proactive approach to the brand image might also lead both internally as externally into a more trustworthy communication of environmental issues.

Furthermore, in Paper 2 the aim to improve the company's competiveness was also found to be a trigger for adopting green initiatives. This trigger is not explicitly mentioned in Paper 1 , but the adoption of green initiatives may create an opportunity to strengthen a company's competiveness in the market. As stated in Paper 2, investments in adopting and improving green initiatives may not only lead to new customers and market shares but may also, as highlighted by one of the case companies, be necessary in the future to simply retain existing customers. The role of green initiatives and their impact on companies in the future can be difficult to predict. However, one case company showed a certain green progress by its stressed desire to become an "industry shaper" with regard to sustainability as well as to other dimensions of its work. Additionally, the strategic priority accorded to green issues can serve as an indicator on a company's level of adoption of green initiatives. All the case companies discussed in Paper 2 mentioned some level of strategic priority (either long-term, short-term or both) regarding green initiatives. The smaller case companies highlighted the importance of having both shortand long-term perspectives, and as one case company stated: "it is not realistic to view environmental aspects and develop strategies and goals from a purely short-term perspective". This is in line with the results from the first literature review in Paper 1, which dealt with sustainable service development literature. Strategic commitment and the 
inclusions of green aspects into existing business systems and strategies are needed to fully integrate sustainability into a company's activities and later on, for the development of the services and products (Waage et al., 2004). Benefits achieved from this could be a more effective cascading of sustainability throughout the company's activities (Maxwell et al., 2006), but the integration of, for example, sustainable service development as one element of the existing corporate strategy, will require new forms of thinking in existing structures and systems (Baumann, 2002).

This in turn, as stressed by Charter and Clark (2008), leads to an increased need to have a clear vision, objective and strategy, supported by a driving force from the top of the company. The top management's role as a trigger is also identified among the case companies in Paper 2. As stated above, a successful adoption of green initiatives, as well as the integration of green considerations into the business strategy, will not occur without clear leadership, resources commitment and active support from the company's top. This in turn, leads to an increased pressure on the top management of LSPs when trying to integrate green thinking at the strategic, tactical and operational level within the company to provide sustainable solutions that benefit all stakeholders.

Finally, the last trigger identified, cost reductions, was not as prominent as the other triggers mentioned in Paper 2. Only two case companies indicated that cost reduction were an influencing factor. One possible explanation to its less importance could be that overall there is already a great focus on reducing costs and at the same time improve performance within the transport and logistics industry. Some of these cost reduction and improvement in performance may have already a reduced impact on the environment, even if this was not the company's original purpose. Besides, as acknowledged by some of the case companies in Paper 2, there are no large cost savings for LSPs themselves, instead it is their customers that stand to save money.

To summarize, the above analysis identifies some possible triggers and the most prominent were Customers, Brand image, Competiveness and Top management. Furthermore, the analysis suggests how the impact of these triggers can affect LSPs when adopting green initiatives in different ways. Customers can, for example, affect the adoption of green initiatives regarding the type of service offerings provided, the segmentation of customers and the strategic partnership possibilities. Brand image and competiveness can affect how LSPs choose to profile the company as well as how they develop strategies regarding green initiatives. Top management was also mentioned as significant, and this trigger can affect the way in which green thinking and initiatives are integrated at the strategic, tactical and operational level within the company.

\subsection{HOW CAN DIFFERENT DRIVERS AND BARRIERS AFFECT THE ADOPTION OF GREEN INITIATIVES?}

The second research question aims to identify how different drivers and barriers can affect the adoption of green initiatives. The position of Research Question 2 as well as its main input is illustrated in Figure 9, below. 


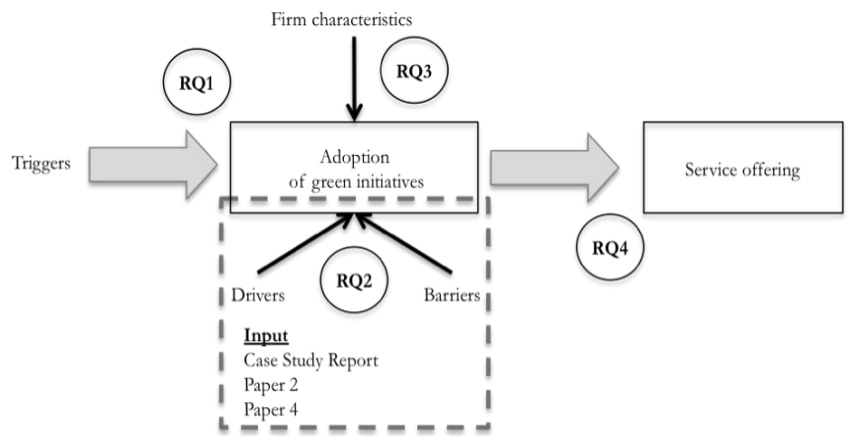

Figure 9 The position of Research Question 2.

Paper 2 deals with different drivers and barriers identified among the case companies studied, while Paper 4 identifies drivers and barriers found from the results by the questionnaire study. The analysis of Research Question 2 is divided into two parts; it begins with how different drivers can affect the adoption of green initiatives and is then followed by a section that analyses how different barriers can affect the adoption of green initiatives.

\subsubsection{How can different drivers affect the adoption of green initiatives?}

One way to identify how different drivers can affect the adoption of green initiatives can be by investigating the role and influence of different stakeholders. Another way can be to investigate the different reasons that drive the adoption of green initiatives. In order to investigate the impact of different drivers on the adoption of green initiatives, the analysis has been divided into the impact of different stakeholders and the reasons identified that drive the adoption of green initiatives. The following analysis starts with the impact of different stakeholders on LSPs when these adopt green initiatives.

\section{How can different stakeholders affect the adoption of green initiatives?}

As a starting point, the results of the impact of the different drivers on green initiatives from Paper 2 are highlighted. Hence, Paper 2 includes not only Swedish-based LSPs (SWE A, SWE B, SWE C and SWE D, which in this licentiate thesis are equivalent to Alfa, Beta, Epsilon and Zeta) but also Italian- and Spanish-based LSPs. Since the analyses in this thesis frame focus primarily on the Swedish market, the following analysis concerns the Swedish case companies and is also extended into two more case companies; Gamma and Delta (a comprehensive description of the case companies included can be found in Appendix 2). However, the results of Paper 2 will nevertheless be used as support to identify any similarities or differences with the updated analysis of the Swedish-based LSPs.

The analysis starts with the drivers identified and studied in Paper 2, including; Customers, Competitors, Government legislation, Management and Employees. Furthermore, the list of different stakeholders in this analysis is extended with Society, Suppliers and Investor/owner, which also were investigated in the case study. The results of these different stakeholders' impact on the adoption of green initiatives among the six Swedish-based case companies are presented in Table 6 below. 
Table 6 The impact of different stakeholders among the studied case companies.

\begin{tabular}{l|llllll} 
& Alfa & Beta & Gamma & Delta & Epsilon & Zeta \\
\hline Customers & High & High & High & High & High & High \\
Competitors & Medium & Medium & Medium & High & High & Medium \\
Government legislation & High & High & Medium & Medium & Low & High \\
Management & High & High & Medium & High & High & High \\
Employee involvement & High & High & Medium & High & High & High \\
Society & High & High & Medium & High & Medium & Medium \\
Suppliers & Medium & Medium & Low & Low & Low & Low \\
Investor/owner & Medium & High & High & High & High & Medium
\end{tabular}

Starting with the customers, Table 6 shows that this driver does seem to have a significant impact on the adoption of green initiatives among the studied case companies. This is in line with the literature that highlights pressure from customer as a significant driver when adopting green initiatives (Foster et al., 2000).

However, this driver showed to have a less impact in Paper 2, since customers' impact was rated low among the Italian and Irish case companies. Even if customers were seen as an important driver among the case companies studied, the findings suggest that there is a certain lack of customer support and willingness to invest in and pay for green initiatives. Hence, the overall perception among the case companies is that customers' interest has increased and the specifications of green requirements have become more realistic over the years. The interest among customers is high, especially among public authorities and companies that have an aggressive sustainability profile. Furthermore, customers from the industry sector are considered to have fewer and lower green requirements than customers from the food industries. A possible explanation to this can be that these customers are closer to the end consumers and therefore feel a higher pressure. The case company, Zeta, stated that its larger customers always have some kind of sustainability requirements, such as ISO certifications or special kinds of transport modes, while requirements from smaller customers are not so clear. Even if the green requirements have become more realistic, Zeta stated that there are still problems due to the diverse of green requirements and difficulties to fulfil these requirements. In order to avoid these problems, some case companies are showing an interest in working more closely with their customers when it comes to green initiatives, and one case company, Delta, stated: "We have to work together with our customers in order to get the best effect". The findings and the reasoning above indicate that the case companies studied are taking their customers seriously and want to meet their demands in terms of green initiatives.

In Paper 2, competitors were not seen as a major driver among the studied case companies. One possible explanation is the fact that many LSPs are still in the initial phase of their development in terms of a green profile and thus sense no pressure from their competitors. However, market research does exist to some extent to benchmark how competitors operate with their environmental work. The fact that competitors can been seen as drivers is underlined by Salomone (2008), who states that they can have a significant impact on motivating companies and bringing the green initiatives' development further to exploit new market opportunities and win new market shares. Some of the case companies believed they are quite far ahead of their competitors when it comes to adopting green initiatives, and in order to maintain that position, they strive to be aware of their competitors' green situation. 
The results indicate that even if the case companies don't sense any competition from their competitors today, this situation may change in the future and to have good sustainability work might even become a competitive advantage.

According to Walker et al. (2008), government regulations and legislation are major drivers of companies' environmental efforts. However, the perception of government legislation's impact varies slightly among the case companies. Government legislations are always present in the background and must of course be followed; otherwise there is a risk of losing customers. Hence, compliance with environmental legislation is no guarantee of improved environmental performance. Instead, it can be seen as a barrier due to weak enforcement (Shi et al., 2008), low levels of awareness and the absence of a central source of information (Balzarova and Castka, 2008). This is partly in line with the general perception of government legislation among the case companies. The case companies state that there are not many legal requirements and that existing requirements are often a bit vague and complicated and therefore difficult to understand. As mentioned in the literature, governments want companies to become greener, but it is not always without complexity to translate general environmental targets to specific requirements for a single company (Roth and Kåberger, 2002).

Despite the vague and complicated legal requirements, the findings indicate that the studied case companies are taking the governmental legislations seriously and one case company, Zeta would even support more and stricter environmental legislation.

One of the most prominent drivers found in Paper 2 was management, which is also in line with the results among the case companies. In the literature management is commonly mentioned as a barrier in relation to the adoption of green initiatives. Reasons provided include managements' resistance to change (Shi et al., 2008) and lack of understanding and awareness from the top management (Post and Altman, 1994). The management is not seen as a barrier among the case companies studied but instead, all the case companies state they have good support from the management and board of directors when it comes to adopting green initiatives. However, in those cases where pressure has not directly come from the top management, it has been a little bit more difficult to persuade the top management to invest in green initiatives. This indicates that management's support and role as an initiator does affect the case companies studied and also reflects the current and future approach to adopting green initiatives within a company.

Likewise as in Paper 2, employees' involvement also proved to have a significant impact among the studied case companies. Furthermore, the significant impact of the role of employees' interest and involvement in adopting green initiatives is also highlighted in the literature (e.g. Berns et al., 2009). Some reflections and comments from the case study regarding the impact of this factor are that it has increased over time due to public awareness about environmental issues, that the level of employees' environmental awareness and knowledge are measured within the company every year and that the employees' involvement in green issues is significant, since some case companies stated a desire to be viewed as an attractive workplace as well as profile the entire company as a green sustainable LSP. This view of integrating green thinking thorough the entire company and involving the employees in this process is especially highlighted by case 
company Beta. Beta characterized itself as a more goal-oriented rather than top-down oriented organization, and stated the following "We work a lot to increase personal responsibility and willingness to contribute to the whole company. We think this is a winning concept".

Other possible drivers that were investigated among the studied case companies were society, suppliers and investors/owners. Even if the studied case companies did not feel any direct pressure from society, there was a desire to be viewed as a responsible green company and not receive bad publicity from the media and thus risk destroying the brand image. As found in the literature (e.g Walker et al., 2008) the pressure from suppliers was not emphasised among the case companies studied. Instead, they considered themselves to have the more active role in pushing for the adoption of green initiatives. When it comes to investor/owner, the influence of this driver has increased. The management has noticed that environmental issues are important, which has generated into increased pressure and set targets.

In addition, the influence from different stakeholders was also investigated in the questionnaire study, and the result was presented in Paper 4. The results from different stakeholders impact when adopting green initiatives is illustrated in Table 7, below. Hence the analysis has been updated as mentioned in section 3.6. As can be seen in the table, the results from the questionnaire study are more or less in line with the findings from the case studies above.

Table 7 Stakeholders' impact on the adoption of green initiatives.

\begin{tabular}{lccc}
\hline & N & Mean & $\begin{array}{c}\text { Std. } \\
\text { Deviation }\end{array}$ \\
\hline Competitors & 60 & 2,8 & 1,38 \\
Customers & 61 & 4,2 & 1,12 \\
Top Management & 61 & 4,4 & 0,92 \\
Transport/Logistics Suppliers or partners & 59 & 3,3 & 1,25 \\
Transport/Logistics Equipment Suppliers & 59 & 3,0 & 1,32 \\
Employees & 61 & 3,4 & 1,29 \\
Experts (academics/consultant) & 56 & 2,3 & 1,35 \\
Trade bodies & 59 & 3,1 & 1,41 \\
Government and public bodies & 56 & 3,2 & 1,50 \\
Owner/shareholders & 61 & 3,5 & 1,48 \\
Insurers & 53 & 2,1 & 1,20 \\
\hline
\end{tabular}

Influence from the top management received the highest mean, and the t-test showed that this was a significantly higher mean than all the other drivers with the exception of customers. Furthermore, the high mean of customers showed a significantly higher influence than that of most other stakeholders apart from top management.

To summarize, the influence of different stakeholders seems to play a significant role in driving LSPs to develop and adopt green initiatives. The most prominent drivers seem to be customers and top management. The significant role played by customers may not be so surprising since without customers' interest and willingness to pay, it is hard to do business. Besides, in order to get the best results from the adoption of green initiatives it may be necessary to cooperate more closely with the customers. The importance of a supportive and committed top management may not either be revolutionary in itself, but 
when it comes to incorporating green initiatives, something which can be seen as a change within the company, the top management's support may be crucial for how successfully the change will be integrated into the company and received by employees. Therefore, the environmental awareness and the willingness to change among the employees may also be relevant when integrating and adopting green initiatives within the company.

\section{How can different reasons acting as drivers affect the adoption of green initiatives?}

The reasons that have been investigated as acting as drivers among the case companies concerned first and foremost cost savings and potential source of revenue. Table 8 shows their impact when green initiatives are adopted among the case companies studied.

Table 8 The impact of the studied reasons acting as drivers.

\begin{tabular}{l|llllll} 
& Alfa & Beta & Gamma & Delta & Epsilon & Zeta \\
\hline Cost savings & Medium & High & Medium & Medium & High & High \\
Potential source of revenue & High & Medium & High & High & Medium & High
\end{tabular}

When it comes to cost savings, it is hard to avoid the fact that this driver both with and without environmental concerns need to be taken into account. In the literature, cost savings are often mentioned as a common driver and Walker et al. (2008) noticed that companies that excelled in various green supply chain activities were partly driven by a strong focus on cost savings. Hence, one case company, Delta, stated that the company did not adopt green initiatives just in order to save money, but that, cost reductions were viewed as a positive side effect. Furthermore, the case companies stressed that it is their stakeholders (e.g. customers and suppliers) that make the largest cost savings. However, Zeta stressed that this driver needs to be taken into account because without a focus to ensure its customers' cost reductions, the company would risk losing customers and market shares to competitors.

Regarding potential source of revenue, Seidel et al. (2009) mention the opportunity to develop new product ranges for environmental concerns. This in turn can attract new customers, and companies may look at adopting green initiatives as a potential source of revenue or cash flow. From the findings in the case study, one LSP, Delta, states that it would probably have lost customers if the company had not invested into green initiatives. Hence, the overall perception among the studied case companies regarding adoption of green initiatives is that it can be a way to win new customers as well as to retain existing customers. Furthermore, the case companies see the potential, and hope that environmental issues will become more important in the future and also that customers will start to show more interest and willingness to pay for green initiatives. This indicates that the adoption of green initiatives is not a temporary trend, instead the studied case companies are taking this problem seriously from a more long-termed perspective.

Paper 4 presents some additional identified reasons that act as drivers that may influence the adoption of green initiatives among LSPs, see Table 9. 
Table 9 Reasons studied that drive the adoption of green initiatives.

\begin{tabular}{lccc}
\hline & $\mathrm{N}$ & Mean & $\begin{array}{c}\text { Std. } \\
\text { Deviation }\end{array}$ \\
\hline Cost reduction for customers & 59 & 3,5 & 1,32 \\
Cost reductions for suppliers/partners & 56 & 3,1 & 1,37 \\
Cost reductions within my company & 59 & 3,9 & 1,26 \\
Improve customer relationship & 60 & 4,3 & 0,86 \\
Improve customer service & 59 & 4,1 & 1,10 \\
Increase firm's competiveness & 59 & 4,4 & 0,88 \\
Increase firm's revenue & 59 & 3,9 & 1,21 \\
Increase ROI & 44 & 3,4 & 1,37 \\
Increase market shares & 58 & 4,0 & 1,15 \\
National and EU laws and regulations & 59 & 4,0 & 1,21 \\
Economic means of control (e.g. taxes, & 59 & 3,4 & 1,37 \\
subventions) & & & \\
Improve brand image & 59 & 4,5 & 0,90 \\
Reduce company risk & 56 & 3,6 & 1,37 \\
\hline
\end{tabular}

As can be seen in the table, most of the reasons studied are thought to have a rather similar and high influence on companies adopting green initiatives. Improve brand image received the highest mean although this driver was not significantly higher than Improve customer relationships and Increase firm's competiveness. Furthermore, the driver Increase firm's competiveness also received a high mean but was not significantly higher than Improve brand image and Improve customer relationships. Regarding Improve customer relationships, this driver had a significantly higher mean than all the other drivers with the exception of Improve customer service, Increase firm's competiveness and Improve brand image.

To conclude, the above analysis indicates that the adoption of green initiatives can be seen as a way to retain and increase the company's competiveness, and according to the survey results, increased competiveness and an improvement of the brand image seem to be significant when LSPs adopt green initiatives. As was shown in the previous analysis, customers do seem to be significantly and by adopting green initiatives the LSPs studied aim and strive to win new customers. Improved customer relations were also something that was significant in the survey results. These reasons relates to the driver Potential source of revenue. Regarding cost savings, it is clear that to some extent this driver both with and without green concerns affects the studied LSPs since in general, transport is often viewed with a great focus on cost reductions (Stank and Goldsby, 2000). The impact of cost reductions is not perceived as the strongest driver (as also the survey results indicated) when adopting green initiatives but as indicated in previous analysis, cost savings have to be taken into account otherwise the company risks to losing customers and market shares.

\subsubsection{How can different barriers affect the adoption of green initiatives?}

The analysis of how different barriers affect the LSPs studied when these adopt green initiatives, has been divided into internal and external barriers.

\section{Internal barriers affecting the adoption of green initiatives}

Based on the observations from the LSPs studied in the case study regarding internal barriers illustrated in Table 10 below, it is shown that financial and economic barriers may have an impact for adopting green initiatives. 
Table 10 The impact of internal barriers among the studied case companies.

\begin{tabular}{ll|llllll} 
& & Alfa & Beta & Gamma & Delta & Epsilon & Zeta \\
\cline { 2 - 7 } Internal & Financial \& economical & High & High & Medium & Medium & High & High \\
\cline { 2 - 7 } & Technical & Medium & High & High & High & High & Low \\
\cline { 2 - 6 } & Information & Low & Medium & High & High & High & High \\
\cline { 2 - 4 } & Organisational & Low & High & Medium & Low & Medium & High
\end{tabular}

Furthermore, the significance of financial and economical barriers, with large investment costs and long payback periods were also identified in Paper 2. Many articles in the literature also point out financial issues as barriers when companies try to meet the increased environmental requirements (e.g. Dahlman et al., 2008, Balzarova and Castka, 2008 and Shi et al., 2008). The small case companies, Alfa and Beta, highlighted the problem that environmental solutions are much more expensive and their financial support is needed, for example from local government to make these investments possible, while the large case companies, Epsilon and Zeta, stressed problems such as low returns and difficulties to specify the expected results and profit. In turn, these problems can have a negative impact when it comes to justifying investments in green initiatives. Among the specialized case companies, Gamma and Delta, this barrier was noticeable, but not to such a great extent as in the other cases. Another contributing factor, apart from the problems mentioned above that may have an impact on why financial barriers are perceived as significant among the studied LSPs, could be that the transport business is a low margin industry.

Technical barriers may also have a significant impact when green initiatives are adopted since four of the six studied case companies stated that this barrier as high. This barrier can be seen both as an internal and an external barrier and as described in the literature can include a lack of technical training, lack of access to external technical support (Shi et al., 2008), uncertainty among existing techniques and inability to eliminate some risks or effects (Post and Altman, 1994). How and in what way this barrier affects the studied case companies can partly be linked to the development and research within the automotive sector. More specifically, the case companies mentioned reasons such as a limited number of environmental friendly vehicles on the market, limited access to refuel these vehicles, hard to find alternative solutions, technology development that is too slow as well as uncertainty among existing solutions. The problems of uncertainty and difficulties regarding finding green technologies solutions on the market may slow down the green development process and can also have a negative influence on LSPs when it comes to justifying investments within their own company. Another technical issue brought up by some of the case companies was the difficulties to measuring and calculating carbon emissions from the transports. The lack of a standardized method and the weak robustness of these calculations may have negative influence on LSPs, since customers may perceive these figures not accurate enough and therefore be unwilling to pay extra for these kinds of service offerings. By that, it is evident that technical and financial and economical barriers tend to go hand in hand and might even reinforce each other.

Like the technical barrier, the information barrier can both be an internal and external barrier, and based on the results from the case study this barrier, seemed to affect the case companies studied adopting green initiatives. This barrier was not very much highlighted in Paper 2, even though ICT systems were seen to be not as supportive and flexible as the 
companies would like regarding green information. This issue concerning inadequate ITsystems is also mentioned among some of the case companies, especially with regard to estimating emissions. The information barrier seems to have a lower impact among the small case companies since they perceived their IT-systems to be sufficient to meet the required need. This situation may be different to that found in a large company. Zeta, for example, stated that due to the large customer base and no standardised green offerings, information could be a problem.

Furthermore, as mentioned in the literature, information barriers can also include difficulties to access and collect appropriate data (Post an Altman, 1994). The difficulties to absorb information about the sustainability impact as well as explain and interpret the information in an objective manner, are also stressed by some of the case companies. In addition, one case company, Gamma, expressed an overall fear of scaring customers off if there is a lack of an entirely reliable data and information base. However, due to efforts to decrease this barrier Gamma has noticed a tendency among its employees and partners to accept the situation. These in turn have identified advantages such as attracting new customers and gaining competitive advantages in price comparison with competitors. This in turn indicates that information sharing and communication may have an impact on how well the adoption of green initiatives is received within the company as well as how it perceived by customers, since the level of green knowledge is probably not much higher among customers. And as case company, Beta stated: "We want to educate our employees so they can support our customers and everyone in the organisation should know where we are standing when it comes to sustainability issues".

Regarding organisational barrier, the perception of its impact varied among the studied case companies and was not identified as a distinctive barrier in Paper 2. Issues concerning this barrier identified in Paper 2 as well as in the case study were lack of understanding and interest in the organisation, different processes in different parts of the company and lack of competence in the area of green logistics. Different methods and approaches, as well as problems with information and cultural differences regarding work with green initiatives concerned first and foremost the large case companies with different functions and global units within the organisation. The reasoning above indicates that organisational resilience is correlated with information dissemination, which in turn shows the relevance for LSPs to be clear about the incentives of adopting green initiatives and approaches used within the company.

In addition, the impacts of different internal barrier were also investigated in Paper 4 and the results are as shown in Table 11 below. 
Table 11 Internal barriers to the adoption of green initiatives.

\begin{tabular}{lccc}
\hline & N & Mean & $\begin{array}{c}\text { Std. } \\
\text { Deviation }\end{array}$ \\
\hline High investments costs & 70 & 3,7 & 1,19 \\
Uncertain payback period & 69 & 3,6 & 1,36 \\
Lack of financial resources & 70 & 2,7 & 1,34 \\
Lack of organisational/human resources & 72 & 3,3 & 1,34 \\
specifically devoted to manage such initiatives & & & \\
Lack of knowledge/skills in-house & 72 & 3,0 & 1,30 \\
Lack of ICT skills internal & 70 & 2,8 & 1,34 \\
Lack of ICT skills external & 67 & 2,1 & 1,10 \\
High ICT running cost & 65 & 3,1 & 1,26 \\
\hline
\end{tabular}

The results reveal that the largest internal barriers experienced by the surveyed LSPs are High investments costs and Uncertain payback period. Furthermore, the barrier High investments cost showed a significantly higher mean compared to all barriers other than Uncertain payback period, which in turn, had a significantly higher mean than most of the other barriers studied (with exception of High investments cost, Lack of organisational/human resources and High ICT running cost).

To conclude, the above analysis investigates some different internal barriers and strives to describe how these affect the adoption of green initiatives among the LSPs studied. The analysis indicates that even if two or more case companies highlight the same barrier, there can still be a variation among the different LSPs about how this barrier will affect the adoption of green initiatives within the company, for example when it comes to informational and organisational barriers. This was also found regarding the financial and economical barriers, which from the results from the case study as well as the survey also seems to be a significant barrier when LSPs adopt green initiatives.

\section{External barriers affecting the adoption of green initiatives}

The external barriers investigated among the case companies concerned policy and market barriers and the impact of these barriers experienced among the studied case companies are shown in Table 12, below.

Table 12 The impact of external barriers among the studied case companies.

\begin{tabular}{ll|llllll} 
& & Alfa & Beta & Gamma & Delta & Epsilon & Zeta \\
\cline { 2 - 7 } External & Policy & High & Medium & Low & High & Low & High \\
\cline { 2 - 6 } & Market & High & High & Medium & Low & High & High
\end{tabular}

As mentioned in the Chapter 2, policy barriers can be described as a weak enforcement of environmental regulations, an absence of economic incentive policies (Shi et al, 2008) low awareness of environmental legislation and also an absence of a central source of information on environmental legislation (Balzarova and Castka, 2008). The perception of the impact of this barrier slightly varied among the studied case companies. Some case companies, Gamma and Epsilon, did not state that policy was a major barrier, since they perceived that no demands are made. But those case companies that mentioned policy as a barrier expressed issues such as questioning set requirements and decisions from government within the company. In addition, some laws and policies require a lot of paper work, which takes a lot of time and leads to extra difficulties as well as pressure to 
follow these requirements, even though the company has no resources for it. This reasoning suggests that the role of the government may be essential when it comes to encouraging the green movement by setting clear standards and requirements and providing approaches for companies about how these requirements will be achieved.

Market barriers as described in Chapter 2, can consist of lack of preferences and demands from customers, as well as weak public awareness and pressure from society (Shi et al., 2008). This barrier seemed to have some impact when most of the case companies adopted green initiatives. One aspect of how this barrier affects LSPs mentioned by Alfa is bad publicity from society, since the transport industry has been negatively labelled as the largest emitter of carbon emissions, and this view is considered difficult to wash off. This in turn can explain why, as identified earlier in this chapter, the LSPs mentioned that improved brand image was a trigger as well as a significant driver for adopting green initiatives.

Even though customers were singled out as a prominent driver, they were also mentioned as a barrier among the studied case companies. Likewise, the lack of customer/market support emerged as a clear barrier in Paper 2. As stated in Paper 2, if customers were willing to pay extra for greener services or at least share the cost of the investment in green solutions, then such services would probably reach the market sooner. But as noticed by one LSP in the case study, Alfa, today these kinds of investments are made in pure good will or by a company's own driving force without any financial gains. Another aspect stressed by one of the case companies, Zeta, is that even if customers have green requirements, the variety and different nuances of these requirements cause problems for LSPs. The case company pointed out that perhaps trade associations could do more in order to reduce these market barriers. In addition, one case company, Beta, mentioned another problem related to requirements from customers. Due to the fact that many customers have separate purchasing and environmental and quality departments, this leads to misunderstandings and risk some sustainability issues never to be discussed and result in the end with requirements unreasonable to achieve. Hence, this may be an organisational barrier but it is also a problem that may affect many companies, and therefore shows the importance of integrating green thinking into the whole company.

Noticeably, the two specialised case companies, Gamma and Delta, did not identify the market barrier as strongly as the other case companies, which in turn can be explained by their overall business strategy. Express deliveries are a bit contradictable towards green thinking but also their customers' requirements might be slightly different to those of the other case companies.

Moreover, in addition to these previously mentioned external barriers, Paper 4 investigated additional external barriers that may have an impact on LSPs adopting green initiatives, see Table 13. 
Table 13 External barriers affecting the adoption of green initiatives.

\begin{tabular}{lccc}
\hline & $\mathrm{N}$ & Mean & $\begin{array}{c}\text { Std. } \\
\text { Deviation }\end{array}$ \\
\hline $\begin{array}{l}\text { Limited access to technology that reduces environmental } \\
\text { impact (e.g. vehicles, aerodynamic features) }\end{array}$ & 65 & 2,91 & 1,34 \\
Lack of customer interest & 71 & 3,2 & 1,36 \\
Lack of customer support & 66 & 3,3 & 1,34 \\
Negative impact on customer supply chain & 63 & 2,4 & 1,20 \\
Lack of transport/logistics suppliers' or partners' interest & 68 & 2,9 & 1,32 \\
Lack of transport/logistics suppliers' or partners' support & 66 & 2,8 & 1,30 \\
Lack of economic incentives & 67 & 3,5 & 1,18 \\
Lack of clear regulations & 65 & 3,3 & 1,30 \\
Lack of ICT vendors selling specific product supporting & 63 & 2,5 & 1,12 \\
green logistics & & & \\
Lack of standards (including ICT standards) & 60 & 3,1 & 1,27 \\
\hline
\end{tabular}

The largest barrier, Lack of economic incentives, has a mean of 3.5, which is significantly higher than most other external barriers (except from Lack of customer interest, Lack of customer support and Lack of clear regulation). Furthermore, the barrier, Lack of customer support, also proved to have a significantly higher mean than most of the barriers apart from Lack of customer interest, Lack of clear regulation as well as Lack of economic incentives.

To summarize, as in the analysis concerning internal barriers, financial issues involved in the adoption of green initiatives seem to be evident both in the cases studied, where the LSPs mentioned customers' unwillingness to pay, as well as in the survey, where lack of economic incentives received the highest mean. The analysis also indicated that not only are customers seen as a significant driver, but they also are mentioned as a barrier among the LSPs studied. This finding is also indicated by the results from the survey, where lack of customer support was singled out as a significant barrier. Regarding environmental legislation, the studied LSPs mentioned problems such as absence of these kinds of regulations and that the few existing regulations are too vague or unclear. Lack of clear regulation also received a relatively high mean in the survey, even if it was not possible to show any statistical significant.

\subsection{HOW CAN FIRM CHARACTERISTIC AFFECT THE ADOPTION OF GREEN INITIATIVES?}

In order to obtain a fair and a more comprehensive picture of the current green situation of LSPs during the research process Research Question 3 has become more and more prominent. The position of Research Question 3 as well as its main input is illustrated in Figure 10, below. 


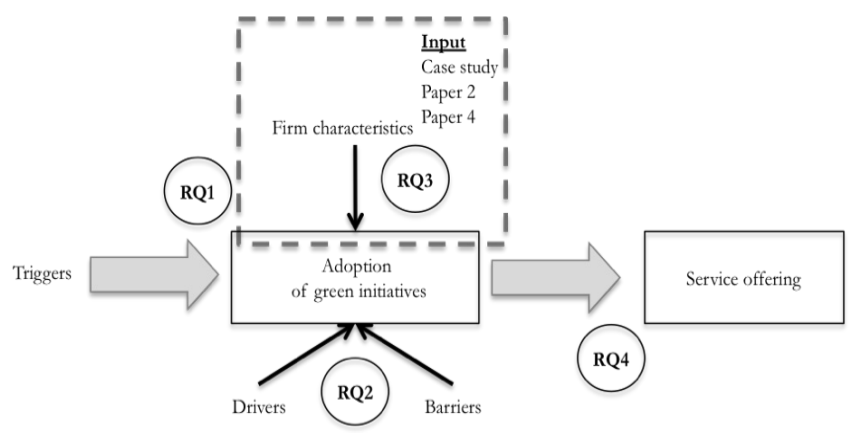

Figure 10 The position of Research Question 3.

Research Question 3 aims to investigate how firm characteristics can affect the adoption of green initiatives. As mentioned in the literature, firm characteristics can have an impact on the adoption of green initiatives within companies. Hence, the analysis of research question 3 may not be considered as exhaustive, but nevertheless regarded as interesting. Focus has been on those firm characteristics that were singled out from the earlier analysis in this thesis frame and also reflected upon in some of the Papers. The two most interesting firm characteristics which this analysis aims to examine more closely, are: the size of the company and the type of service provided.

In the literature, the size of the company is noticed to be one of the most prominent firm characteristics and research has shown that the number of employees, company history and capital size do influence initiatives undertaken by LSPs (e.g. Lin and Ho (2008) and Ho et al. (2009). The results from the previous analysis, as well as findings from Paper 2 and Paper 4 suggest that among the LSPs studied there are some differences between small and large companies. The differences identified are not only that the perceived impact of various drivers and barriers in some cases might vary between small and large LSPs, but also the fact that a common identified driver or barrier may affect small and large LSPs in different ways.

One barrier, which has been identified as significant for both small and large LSPs but in a slightly differed way for the two groups, was the financial and economical barrier. Paper 4 showed that cost for investments and an uncertain payback period were the strongest barriers identified. One explanation to this may be that most of the respondents in the survey were SMEs and these companies may experience and financial issues as a stronger barrier than a larger company. This in fact might be true, and the findings from Paper 2 show that this barrier was relevant for all the studied LSPs. In agreement with the results of Paper 2, the analysis presented in section 5.2.2 indicated that financial barriers are significant both among small and large LSPs. However, this barrier affected the two groups in different ways. The small case companies, Alfa and Beta, highlighted problems such as green solutions being much more expensive and that for them, financial support is needed from for example local government in order to make these investments possible. The large case companies on the other hand, stressed problems such as low returns and difficulties to specify the expected results and profit. This in turn can lead to difficulties when it comes to justifying investments for green solutions within the company even if they can be afforded. 
This problem within the larger LSPs is somewhat contradictory to the results in Paper 2 that indicated that three out of four large LSPs stated top management as a main incentive (which is mentioned as a trigger in this licentiate thesis) when starting to adopt green initiatives. This finding suggests that green initiatives are embraced at a high level within larger companies while green awareness is also derived from a lower level within smaller companies. Moreover, the large LSPs in the case study also mentioned good support and pressure from the top management. Hence, due to the above analysis, one might wonder; despite good intention how much is the top management really willing to invest into the adoption of green initiatives.

Another interesting finding in Paper 2 concerned the strategic priority accorded to the adoption of green initiatives. While green aspects were considered mainly as a strategic and long-term priority, three out of the four small LSPs also focused on the short-term perspective. One explanation for this might be that smaller LSPs in general are more keen to meet customer's immediate requirements than a larger company that perhaps instead focuses on building longer-term strategic market positions. Furthermore, it was also shown in Paper 2 that the LSPs that claimed that top management was a trigger (most large LSPs) for adopting green initiatives also had a stronger focus on more long-term priorities.

Moreover, another issue that showed to be different between small and large LSPs was the information barrier especially regarding IT-solutions. The result from Paper 2 showed that the smaller LSPs used more simple self-developed tools, while the larger companies often with a wider range of service offerings tended to use more sophisticated software and applications, especially in the transportation area. In the literature, recent research has focused on the ICT adoption among LPSs (see for example Marasco, 2008) and utilization of ICT has increasingly been considered as one success factor (Langley et al, 2005). Despite this, Evangelista and Sweeney (2006) stress that there still is a lack of ICT expertise and insufficient financial support among LSPs, especially among small- and medium-sized firms. Hence, as is shown in the analysis in section 5.2.2 the information barrier seems to have a lower impact among the small case companies since they perceived that their IT-systems are more or less sufficient to meet the required need. The larger case companies perceived that their ICT systems were not as supportive and flexible as the companies wished for, especially with regard to estimating emissions. This issue was however mentioned by some of the other case companies as well. Another big problem mentioned by the large case companies were difficulties with information sharing and spreading both within the company and to customers. Paper 2 also highlighted this issue, and stressed that due to different processes in different parts of the company there is a need for information dissemination. This may be true for all companies, but the large ones are perhaps more vulnerable to difficulties regarding information spreading than small companies.

In the literature, there is a lack of research investigating the connection between adopting green initiatives and other relevant firm characteristics besides size. Hence, from a broader perspective, the purchasing literature for example indicates that companies in different industries experience different hindrances and drivers, which lead them to apply different practices (Zhu and Sarkis, 2006). This in turn suggests that the drivers and 
barriers experienced by LSPs can be dependent on characteristics such as type of product and service provided. The analysis in section 5.2.2, showed that the two specialised LSPs, called Gamma and Delta in the case study, did not experience financial barriers to be as significant as the other case companies did. This could be due to reasons such as either no recent large investment or no difficulties to justify investments regarding green solutions within the company. Furthermore, the two specialised LSPs did not identified market barriers as strongly as the other case companies. This in turn can be explained by the fact that these companies' overall business strategy with express deliveries is a bit out of line with green thinking, and this makes these actors less affected by different pressures from the market than the other LSPs. In addition, these actors' customers and their requirements might also be slightly different than those of the other case companies. For example, the main reason to use express services is not the fact that these services are viewed as having a less negative impact on the environment.

In line with the reasoning above, another interesting firm characteristic, besides type of service provided, can be the company's customer base. As mentioned in the earlier analysis, customers are perceived as a main driver for adopting green initiatives. But the analysis also showed that customers from different industries put various pressure and requirements on LSPs regarding green solutions. For example, as mentioned earlier in the analysis, customers from the industry sector are not as advanced as customers from the food industries when it comes to have more detailed green requirements. This in turn, can be explained by the fact that these customers are closer to the end consumers and therefore feel a higher pressure. Previous analysis also indicated that there might be a difference between small and large customers' requirements as well. For example, Zeta mentioned that larger customers always have some kind of green requirements such as ISO certifications or special kinds of transport modes, while requirements from small customers are not so explicit.

To conclude, as mentioned at the beginning in this section, the analysis of Research Question 3 should not be considered exhaustive; instead it suggests some examples of firm characteristics that might affect LSPs when adopting green initiatives. Not only does the size of the company seem to play an influential role but also the type of services provided and the type of customers seem to influence LSPs adopting green initiatives. The discussion above indicates that firm characteristics play an important role regarding how drivers and barriers may affect the adoption of green initiatives. From a longer perspective, these firm characteristics may also have a significant impact on the further development and strategic direction regarding green initiatives undertaken by LSPs.

\subsection{IN WHICH WAYS CAN THE ADOPTION OF GREEN INITIATIVES BE REFLECTED IN THE SERVICE OFFERING?}

The purpose of the fourth and the last research question is to find out in which way the adoption of the green initiatives can be reflected in the service offering. The main input for this research question comes from Paper 3 but the results of this paper have also been compared with the literature findings from Paper 1. The position of Research Question 4 is illustrated in Figure 11, below. 


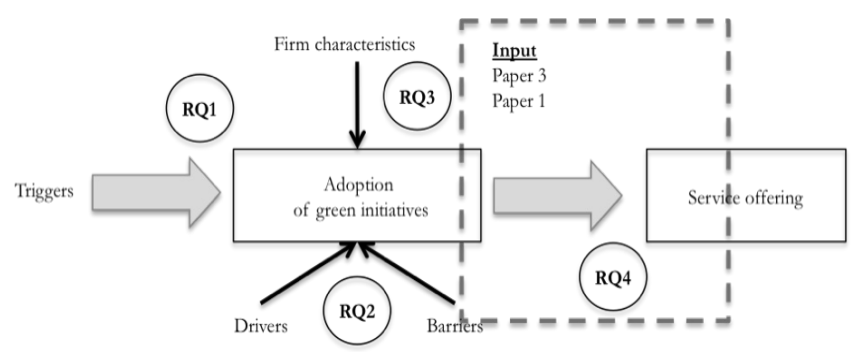

Figure 11 The position of Research Question 4.

As mentioned earlier in section 3.6, the analysis of this research question may be considered to have a more descriptive approach. Despite the fact that the studied case companies have been selected due to their proactive approach to green initiatives, they are still in an early phase of their adoption and thus have no elaborated approach to how this should be reflected in their service offerings.

Starting in the literature, Paper 1 provided a framework that was partly used in order to analyse Research question 1 (see section 5.1). However, the analysis for this research question focuses on the parts of the framework which show how the six aspects identified can be considered in the development of the offering and how these can be visualized and reflected in the offering from a green perspective, see Table 14 below.

Table 14 Parts of the framework presented in Paper 1.

\begin{tabular}{|c|c|c|}
\hline Aspects & $\begin{array}{l}\text { Considerations in the development of } \\
\text { the offering }\end{array}$ & The offering \\
\hline \multirow[t]{3}{*}{ Customer \& market } & Customer demands as starting point & Diversified \& customised offers \\
\hline & Early integration of marketing & Pricing the green service offering \\
\hline & & Customer segmentation \\
\hline \multicolumn{3}{|l|}{ Collaboration } \\
\hline \multirow[t]{3}{*}{ Internal } & Use of cross functional teams & \\
\hline & Include environmental experts & \\
\hline & Identify collaborations risks & \\
\hline \multirow[t]{4}{*}{ External } & Develop methods to handle risks & Show awareness \\
\hline & Collaborate with suppliers & Show willingness to collaborate \\
\hline & Collaborate with customers & \\
\hline & Introduce the collaboration early & \\
\hline \multirow[t]{2}{*}{$\begin{array}{l}\text { Business management } \\
\& \text { strategy }\end{array}$} & $\begin{array}{l}\text { Integrate strategic, tactical \& operational } \\
\text { level cross the company }\end{array}$ & $\begin{array}{l}\text { Hard to visualise in the offering but } \\
\text { might be done with the use of certain } \\
\text { certifications and standards }\end{array}$ \\
\hline & Support from top management & \\
\hline \multirow[t]{4}{*}{ ICT } & Internal measuring & \\
\hline & Collect and store environmental data & Provide customised offerings \\
\hline & & $\begin{array}{l}\text { Display/measure environmental } \\
\text { impact }\end{array}$ \\
\hline & & Facilitate collaboration \\
\hline \multirow[t]{2}{*}{ Means of control } & Focus on outcome not method & Use labelling/standards in the offering \\
\hline & Focus/select e.g. standards & Trustworthy \& informative offerings \\
\hline \multirow{3}{*}{$\begin{array}{l}\text { Competence } \& \\
\text { knowledge }\end{array}$} & Training \& development & Knowledge as part of the offering \\
\hline & $\begin{array}{l}\text { Adjust knowledge needs to strategy and } \\
\text { ambitions }\end{array}$ & Trustworthy offers \\
\hline & $\begin{array}{l}\text { Identify potential knowledge exchange with } \\
\text { external actors }\end{array}$ & \\
\hline
\end{tabular}


The results of Paper 1 are now going to be compared with the empirical findings presented in Paper 3. However, this comparison concerns first and foremost the first analysis of Paper 3, which is mostly empirically driven and is divided according to the six dimensions describing a service innovation suggested by den Hertog et al. (2010). These six dimensions are the following;

- New service concept or service offering

- New customer interaction

- New value system/business partners

- New Revenue model/pricing

- New service delivery system; organisational, personal and culture

- New service delivery system; technological

The first of the dimensions mentioned namely, New service concept or service offering, can be connected the to the first aspect found in Table 14, Customer \& Market. The literature presented in Paper 1, described the importance of diversify and customizing green service offerings. However, the Paper also stressed the importance of knowledge regarding the extent to which these services can be customized but the benefits to customizing these services are not discussed. Paper 3 showed that none of the studied LSPs stated that they offered any specific green logistics service at present. This in turn can be explained by the fact that in general, logistics service offerings have become more customised, due to varying and different logistics needs from different kinds of companies (Hertz and Alfredsson, 2003). Instead, some LSPs studied offered separate green choices connected to their general service offerings, while others tried into integrate green thinking throughout the company and its service offerings. Therefore, the LSPs studied were characterized as either offering green choices or a full integration of green thinking in the company and its service offering.

New customer interaction can also be linked to the aspect Customer \& Market as well as to the aspect Collaboration (external) in Paper 1. As shown in the earlier analysis of this Chapter, the role of the customer as a driver seems to be significant when LSPs adopt green initiatives. Paper 1 suggested how customers can be taken into consideration and thereby be reflected in the service offering. For example, by more interaction with customers, LSPs can increase their understanding of their customers' business, needs and demands, and thereby support their innovation and the development towards more diversified logistics solutions and to a segmentation of customers (based on green awareness, ambitions and willingness to pay). The empirical input from Paper 3 showed that interaction with customers varied among the LSPs studied even with regard to the regular services provided. This is however also reflected to some extent in how they interact about green issues. Furthermore, the analysis in Paper 3 suggested that LSPs could be categorised on the basis of whether they interact on green issues with all customers - the market in general - or a specific customer segment with a special interest.

In the way it is described in Paper 3, the third dimension, New value system/business partners, can be connected to the aspects Competence \& knowledge as well as Collaboration (external) in Paper 1. The literature presented in Paper 1 suggested that there is a need for collaboration with partners as well as an identification of potential knowledge exchange 
with external actors regarding green initiatives. This in turn may be reflected in the service offering with the provision of more trustworthy service offerings and the offering of green knowledge or education as part of the service offering. Among the LSPs studied, some highlighted networking with other companies and organisations as a valuable source for input and ideas, while others just want to keep an overall picture of their competitors' situations. Therefore, from the findings of Paper 3, how LSPs cooperate with external partners in order to exchange green knowledge can be categorised on the basis of: networking with competitors as well as others (such as authorities and research institutions) versus staying aware about the surrounding competition.

The fourth dimension, New Revenue model/pricing, is only slightly touched upon in Paper 1. However, the aspect Customer \& Market, reflects how the adoption of green initiatives can be reflected in the pricing of the service offering. Moreover the Paper also stressed that there is a lack of methods for how to integrate green issues into the pricing of service offerings. In line with these results, Paper 3 showed that in general, there were few traces of price models among the LSPs studied that take green issues into account. Hence, another interesting finding in Paper 3 was that some LSPs see the cost caused by green investments as a way to increase market shares through increased value, while others are careful to keep track of the competitors' pricing in order to meet the market prices. This led to the LSPs being categorised either by existence of price models or motives to invest in green aspects.

New service delivery system; organisational, personal and culture can more or less be related to the aspects Business management \& strategy and Collaboration (internal) presented in Paper 1. As mentioned in Paper 3, it was noticed that organisational encouragement and the quality of human resources have positive influences when green innovations are adopted among LSPs (Lin and Ho, 2008). The results from Paper 3 showed the significant role of employees as co-creators of green value, as well as the importance of a driven and committed board. The importance of offering employees training and education to increase their green awareness and knowledge, as well as the significance of a supportive top management, are also highlighted in Paper 1. This in turn led to the observation that enforcement of green initiatives among the studied LSPs was either done from an employees' perspective (bottom-up) or from a management and board perspective (topdown). However, this can be hard to visualise in the service offering, but Paper 1 suggested that the use of green certificates and standards might make it easier to include and facilitate a trustworthy communication of green initiatives as part of the offering.

Furthermore, Paper 1 stressed the relevance of functional co-ordination and collaboration when green aspects are included in the service offering. However, this was not mentioned in the logistics literature part of the Paper. The results in Paper 3 showed that some LSPs worked more broadly and created a common awareness within the company (broad penetration), while in others companies, different departments adopt different roles (functional specialisation).

The sixth and last dimension, New service delivery system; technological, can perhaps not surprisingly be connected to the aspect ICT, especially since Paper 3 primarily focused on ICT development for this dimension. In general, ICT is described as an important tool in 
the customization and differentiation of the offering (see for example Dobie (2005) and van Hoek and Chong (2001)) as well as in the collaboration with customers (e.g. Bourlakis and Bourlakis (2005) and Mortensen and Lemonie (2007)). In addition, Paper 1 suggested that ICT can be an important facilitator in the inclusion of green aspects into the service offering and that ICT can be used to customize these kinds of offerings. The empirical findings in Paper 3 showed that all case companies had systems in place that to some extent supported their green needs. Clinton (2008) stated that in general, it is important for LSPs to continually invest in ICT in order to meet increasing customer demands. However, the results of Paper 3 of LSPs ambitions of ICT investments for the near future seemed to differ slightly. While some wanted to develop quite fast to meet increasing customer requirements, others processed more slowly but in a moderate pace while some even took a more wait and see approach where no specific action was taken or further investment mentioned. Based on the results of Paper 3, LSPs were categorized with regard to the adoption and development of technology focusing on ICT as fast, slow or awaiting.

The analysis above presents a broad picture of the current status of the greening process of the LSPs studied, where context and approaches to address and adopt green initiatives, as well as how these reflect on the service offering differed among the case companies. Regarding the dimensions studied (in Paper 3) and main aspects (in Paper 1), one dimension can be seen to have a more direct reflection on the service offering, namely New service concept or service offering while the others have a more indirect impact and therefore are not reflected or visualised very much in the service offering.

Furthermore, despite the fact that this is an early phase of LSPs' greening process from the second part of the analysis in Paper 3, it can be discerned that there are three different patterns that reflected the current behaviour and the anticipations among the LSPs studied. These were:

- Focus and abide versus network and explore. This pattern focused on the strategic standpoint of the greening of LSPs' service offering as well as the competitive awareness of the development of the logistics market.

- Internal focus for general "spread" versus broad engagement to target specific customers. The second pattern involved customer orientation and segmentation related to how transferring and sharing green knowledge and competences was organised within the company.

- Top-controlled passiveness versus bottom-up exploration of technology. The third and last pattern concerned how green initiatives are initiated as well as managed and controlled within the company in relation to the company's attitude towards green investments.

To conclude, as pointed out in the beginning of this section, the analysis of the fourth research question can be considered to be a little bit more descriptive due to the fact that the adoption of green initiatives and how this reflects on the service offering is still in an early phase among LSPS. Although the analysis indicates that some LSPs are more advanced than others, there is, as mentioned in Paper 3, an overall tentativeness concerning the future green process. Even though all of the selected LSPs have adopted 
green initiatives, there seem to be differences in how LSPs act and reason. The differences in LSPs' reaction and adoption of green initiatives can possibly be explained by the nature of the LSPs businesses. However, the identified patterns did not separate the LSPs studied in similar ways why it is hard to generalise it to firm characteristics. Finally, the green approach selected and the ways LSPs choose to react and adopt green initiatives will in the end also most likely be reflected in their service offerings. 


\section{CONCLUSiOnS AND FURTHER RESEARCH}

This chapter begins with a concluding discussion based on the findings from the different research questions with the aim of answering the purpose of this licentiate thesis. Thereafter, the contribution of this thesis, both of academically and practical relevance, is discussed. The chapter finishes off with a conclusion about further research suggestions within the thesis' area.

\subsection{CONCLUDING DISCUSSION}

Based on the analysis, it is reasonable to suggest that adoption of green initiatives not is viewed as a short-lived trend among the studied LSPs, or as an established practice. Instead, it can be described as a potential business opportunity. In the long run, adopting green initiatives may have a positive effect on the company's image and brand. On the other hand, if LSPs remain inactive in regards of adoption to green initiatives, it can increase the risk of being exposed of criticism and negative press, as well as increase the fear of lagging behind competition. As turned out in the analysis, the studied LSPs have commenced to adopt green initiatives since they experience the future pressure, understand the consequences and realize the necessity in order to respond to the increasing and more global competition. In the literature, this is identified as a main aspect for a business change (Lemoine \& Skjøtt-Larsen, 2004).

The findings provide descriptions of how different factors can affect the adoption of green initiatives among LSPs and also how the implementation of green initiatives can be reflected in the service offering. The following section strives to answer the different research questions in a chronologic order.

Starting with the triggers, they provide an increased understanding of what it all comes down to when LSPs start to adopt green initiatives. Here, a trigger is viewed as an incentive that starts the adoption of green initiatives among LSPs. Possible triggers have been analysed and discussed in order to sort out how they can affect LSPs when starting to adopt green initiatives. It turns out that customers do seem to play a significant role as triggers for LSPs adopting to green initiatives. According to the studied case companies, the customers' curiosity regarding green initiatives have increased during the recent years, even if the findings suggest that there is a certain lack of customer willingness to invest in and pay for green initiatives. Top management decisions are another identified trigger for LSPs to start adopting green initiatives. As highlighted both in the literature as well as noticed by some of the case companies, a successful adoption of green initiatives and integration of green considerations in the business strategy will not occur without a clear and active support from the company's top management.

Furthermore, increased competiveness and increased brand image are also mentioned to act as triggers. Notably, even if increased competiveness seems to act as a trigger, the role of competitors as a driver affecting the adoption of green initiatives are neither stressed in the case study, nor being identified as a significant driver in the survey study. On the other hand, there is a general fear among the studied LSPs that if they do not start to adopt green initiatives customers will probably choose and favour other LSPs with a more 
"green" profile. As a consequence, the company could face the risk of loosing market shares. One of the case companies even states that it wants to act before too many customers demand green offerings. By doing so, the company could create an advantage of having a superior standing among competitors, in addition to being a role model by adopting green initiatives.

Furthermore, a variety of different drivers have been investigated with focus on how these can affect the adoption of green initiatives among the studied LSPs. Most of the studied drivers seem to some extent to affect the adoption of green initiatives among the case companies. Hence, it turns out that many of the triggers that "started the adoption of green initiatives" also play a significant role as drivers in the further process as well. For example, customer and top management both have been mentioned as strong drivers. By adopting green initiatives, LSPs strive to win new customers and there is also a desire to improve customer relationships. It is also suggested that the clearer the customers state their green demands the easier it becomes for LSPs to respond to them. Regarding the top management, the analysis indicates that the engagement and support from top management can be crucial for how successfully the adoption of green initiatives are integrated into the company and received by the employees. The employees are not identified as a trigger but employees' involvement turns out to be an affecting driver when adopting green initiatives. The employees' involvement regarding green initiatives has increased over recent years and efforts are made among the LSPs to increase green awareness and knowledge among employees. The conclusion is that among LSPs their employees can be considered as an essential driver and resource when adopting green initiatives.

Besides from how triggers and drivers affect the adoption of green initiatives among the studied LSPs, different barriers and their influence on adoption of green initiatives have been studied. As for the drivers, all different barriers may to some extent affect the adoption of green initiatives among the studied LSPs. However, based on the findings from the case study as well as the results from the questionnaire survey, financial and economical barriers have shown to be crucial when LSPs adopt green initiatives. Uncertain payback period, high investment cost, increased need for external financial support as well as difficulties to justify investment regarding green initiatives within the own company are examples of this mentioned barrier. In the survey study, lack of economic incentives was singled out as the most significant external barrier. In addition, the analysis does not only indicate customers as a significant driver affecting the adoption of green initiatives, but customers are also perceived as a barrier among the studied LSPs. The studied LSPs mention reasons such as lack of customer support, unwillingness to pay for green initiatives, and unclear and unreasonable green requirements from customers as examples of barriers. Lack of customer support was also highlighted in the result from the questionnaire survey.

Before proceeding to how different firm characteristics can affect the adoption of green initiatives, another finding worth mentioning is the role of government legislation. In the literature, government regulation and legislation is described as a major driver of companies' environmental efforts (e.g. Walker et al., 2008). Some case companies mention it as a driver due to the fact that government legislations need to be followed in order for 
the company to be able to act on the market and not risk loosing customers. But at the same time, it was also perceived as a barrier due to the fact that there are few legal requirements, and these are often a bit vague and complicated. This concludes the importance of the government's role in the further process of developing more and clearer green targets and requirements.

A further conclusion of the analysis is that size of the company and type of service offerings provided seem to play important roles when adopting green initiatives. Furthermore, type of customers also seems to affect LSPs in how different drivers and barriers are experienced within the companies. For example, the analysis interpreted that customers from different industries put various pressure and requirements on LSPs in regards to green initiatives and solutions.

Another interesting aspect emphasized in the analysis, originally presented in Paper 2, concerned the strategic priority of the adoption of green initiatives. While green aspects were stated as a strategic and long-term priority, three out of the four small LSPs also focused on the short-term perspective. A possible explanation to this may be that in general, smaller LSPs are more keen on meeting customer's immediate requirements compared to larger company that instead rather focus on building longer-term strategic market positions.

The case companies presented in this study are only examples of different kinds of LSPs that adopt green initiatives. Hence as indicated in a portion of the findings from the analysis, different companies have different basic conditions and are therefore affected by different drivers and barriers in various ways when adopting green initiatives. In other words, it seems like firm characteristics are related to how certain drivers and barriers affect the LSP, which in the end also affect how green initiatives are adopted within the company. The thesis focuses on the firm characteristics singled out from the analysis, which also has been reflected upon in some of the previous papers. Hence, due to the fact that no statistical analysis has been done in this area, the results should be treated with some carefulness. Instead, the results should be regarded as suggestions of firm characteristics that may affect LSPs when adopting green initiatives.

From a more overall perspective, it does not matter what kind of business change or decision a LSP faces, the response to a new challenge will most likely depend on what kind of business the company are running, and what different requirements that the company itself and its customers may have. This implies that type of business as well as requirements from the LSP itself and its customers should not be ignored when it comes to adopting green initiatives. Different LSPs may have similar benefits by adopting green initiatives but how these are achieved depend on the LSPs specific firm characteristics in combination with its business context. Therefore it may require unique solutions adjusted for specific business conditions, as well as customers' needs and requirements. As a result, the difference in LSPs' adoption of green initiatives can therefore be reflected into the service offering.

The results from the different parts in the analysis taken together suggest that firm characteristics rather effect the adoption of green initiatives indirectly than directly. While 
the analysis indicated how firm characteristics can moderate the effects of drivers and barriers, no patterns were identified regarding firm characteristics and the development of green service offerings. The development of green service offerings includes the internal work as well as the explicit service offering.

The analysis mainly focused on six different dimensions describing a service innovation suggested by den Hertog et al. (2010). For each of the different dimensions, a categorization was carried out based on how the studied LSPs reacted upon each of the investigated dimensions. The results indicated that the dimension, "New service concept or service offering", had a more direct influence of the service offering whilst the other dimensions had a more indirect impact and therefore were not that clearly reflected or visualised in the service offering. Furthermore, despite the early phase of LSPs greening process, the analysis presented three different patterns that reflected the current behaviour and anticipations among the studied LSPs. These findings were also reflected in Paper 3. As already mentioned earlier, the differences in the studied LSPs reaction and adoption of green initiatives can partly be explained by the firm characteristics and the nature of the business. Hence, as suggested in the analysis, the selected approach and how LSPs choose to react and adopt green initiatives will in the end also be either directly or indirectly reflected in the service offering. This raises some questions to be further addressed: What is really a green service offering from LSPs? What explicitly is communicated to the customers as a service offering, or does it embrace the LSP company as a whole?

\subsection{CONTRIBUTIONS}

The contributions of the findings of this licentiate thesis can theoretically both be linked to the logistics and to the service marketing research areas. More specifically, the contribution to the logistics area can be viewed both from a general logistics and a green logistics perspective, while the contribution to service marketing area primarily concerns new service development.

The logistics and transport industry has over the last year undergone fundamental changes, which have led to the fact that individual players have faced new challenges of strategic and structural nature (Persson and Virum, 2001). A recent challenge affecting this industry is the response to the negative impact on the environment partly caused by this sector. Therefore, the contribution of this thesis can be viewed from a general logistics perspective describing how some examples of LSPs react and respond to the market conditions and challenges regarding a business change, which in this case is the challenge to greening their companies.

When it comes to green logistics literature, most writing and empirical studies regarding sustainability in Supply Chain Management have originally focused on manufacturing companies (e.g. Eltayeb and Zailani, 2009; Hong et al., 2009 and Srivastava, 2007). Due to this, the licentiate thesis does make a contribution only by addressing the LSPs perspective, which primarily not has been in focus when it comes to green logistics research. As a consequence, since LSPs have been neglected in the prior research, the descriptions of the studied LSPs' greening status can be seen as a contribution by itself. Thus, the identification and investigation how different factors (mentioned in thesis as 
triggers, drivers, barriers and firm characteristics) affect the adoption of green initiatives among LSPs offers additional information to the green logistics field. These results can for example be of special interest when it comes to determining potential factors influencing the willingness to adopt green innovations for the service sector, also mentioned by Lin and Ho (2008).

As stated in the literature review in Paper 1, there seems to be an absence of green aspects taken into account in the general logistics offering literature. A recent review of Rajesh et al. (2011) regarding service offerings provided by 3PLs, further proves that green aspects are neglected in these kind of literature. The results concerning customers' increasing interest in green initiatives as well as the considerations on how the adoption of green initiatives can be reflected in the service offering may therefore put some additional insights to the general logistics offering literature.

Further, how the adoption of green initiatives can be reflected in the service offering can be relevant to the service marketing area, for example in the new service development literature. Mentzer et al. (2001) highlight the importance of close interrelationship between marketing activities and logistics activities, and the results from this thesis can be seen as an exemplification of the linked areas of service marketing and logistics. This study does provide empirically examples of the possibilities to link the development of logistics service offerings to new service offering, which should be considered as beneficial input to the focused area of green initiatives.

Finally, from a practical and managerial perspective, the identification and analysis of different drivers and barriers may be helpful for LSPs to evaluate their own greening situation. By the suggested patterns that reflect the current behaviour and anticipations towards a green approach, LSPs can match their own business and context with the different patterns and its alternative rationales. The findings should also be seen as an inspiration in the continuing work to integrate and adopt green initiatives into the company, and the service offering. In addition, the description of how LSPs respond to and act upon the challenges when adopting green initiatives will also be of valuable information for a variety of stakeholders. For example, it increases the knowledge among society and government regarding the current situation of LSPs today. From a customer perspective, the findings enhance the awareness among LSPs' customers about their central and important role in the greening process. It also facilitates LSPs to convince their customers of the need to work together in order to overcome some of the stated barriers found in this licentiate thesis.

\subsection{FURTHER RESEARCH SUGGESTIONS}

In this section, based on the results from this study, some directions for further research are presented.

The research in this licentiate thesis has focused on examples of different LSPs and has strived to describe how different factors can affect the adoption of green initiatives and how green initiatives can be reflected in the service offering. Due to the relative small sample of case companies, it is not possible to make any generalised assumptions of the LSP industry. As mentioned in Chapter 3, a survey study has also been conducted. Hence, 
the results from this survey study have only been used in this thesis in order to validate the results from the case study. Thus, it would be interesting to compare the results found in this study more circumstantially with the results derived from the survey study since this would enable a further generalisation of the current green situation of the LSP industry.

Moreover, since this study mainly focuses on LSP active on the Swedish market, it would of course be interesting to conduct similar studies in other countries in order to find out if and possibly how LSPs green situation differs between different nations. It would provide a basis for better understanding of this increasingly important area of logistics and Supply Chain Management. What is worth mentioning is also that, the research has been a study of a more "snap-shot" character of LSPs adoption of green initiatives. It would of course also be much interesting to conduct a similar study in some years from now in order to, in a retrospect, investigate the development of LSPs adoption of green initiatives.

Another area to further investigate concerns the question how LSPs will satisfy their customers' demands regarding green requirements. Based on the findings in this study, customers' needs and requirements are seen as a prominent driver of green initiatives among the LSPs, but at the same time these evolving requirements are perceived to act as a barrier. This indicates the need of further exploring how LSPs align their green initiatives with customer requirements. It would be appealing to add the perspective of customer in order to develop a better understanding of the broader dynamics of the green logistics market. By doing so, it would create a clearer view of the factors affecting the adoption of collaborative green initiatives between LSPs and their customers.

Based on the results in this thesis, it seems that LSPs are undergoing a challenging business change, where the traditional view focused on cost and service optimization has started to develop into an additional factor, green aspects, that needs to be taken into account. Therefore, it would also be interesting to study how the impact and relationships between these three factors are perceived and evaluated among LSPs' customers in the purchasing process of transport services. Previous research has put green considerations at the bottom of the priority list (e.g. Björklund, 2005), however there are signals that this may not be case in the future.

The research in this thesis regarding how green initiatives can be reflected in service offerings opens up for a variety of further interesting research questions and directions. For example, it would be interesting to study the service development process in general among LSPs to increase the knowledge of what kind of aspects that influence this process. As Hertz and Alfredsson (2003) point out, to add customer value it is necessary to develop skills and competencies that are superior compared to customers. This leads to questions such as; what kind of green skills and competencies are developed within LSPs, how do they work in order to visualize this in the service offerings to their customers, and how do the customers perceive this?

Conclusively, a central question that needs to be addressed is if adoption of green initiatives is seen as a way to create value? And in such case, what kind of needs the 
customers of LSPs strive to fulfil by using "green" service offerings, and how this influence the perceived value of such type of service offerings? Besides the service offering, the approach of how the adoption of green initiatives affects the relationship and collaboration between the LSP and its customers is an interesting aspect to investigate further. Can adoption of green initiatives result in an even more close relationship between LSPs and their customers, and what kind of business opportunities may this led to in the future? 


\section{REFERENCES}

Andersson, D. \& Norrman, A. (2002), "Procurement of Logistics Services - A Minutes Work or a Multi-Year Project?", European Journal of Purchasing \& Supply Management, Vol. 8., No. 1, pp. 3-14.

Ashenbaum, B., Maltz, A. \& Rabinovich, E., "Studies of Trends in Third-Party Logistics Usage: What Can We Conclude?”, Transportation Journal, Vol. 44, No. 3, pp. 39-50.

Bagchi, P. \& Virum, H. (1996), "European Logistics Alliances: A Management Model", The International Journal of Logistics Management, Vol. 7, No. 1, pp. 93-108.

Balzarova, M.A. \& Castka, P. (2008), "Underlying mechanisms in the maintenance of ISO14001 environmental management system", Journal of Cleaner Production, Vol. 16, No. 18, pp. 1950-1957.

Baumann, H., Boons, F. \& Bragd, A. (2002), "Mapping the green product development field: engineering, policy and business perspectives", Journal of Cleaner Production, Vol. 10, No. 5, pp. 409-425.

Berglund, M. (2000) Strategic Positioning of the Emerging Third-Party Logistics Providers. Dissertations No. 45. Linköping Studies in Management and Economics, Linköping University.

Berglund, M., van Laarhoven, P., Sharman, G. \& Wandel, S. (1999), "Third-Party Logistics: Is Rhere a Future?", International Journal of Logistics Management, Vol. 10, No. 1, pp. $59-70$.

Berns, M., Townend, A., Khayat, Z., Balagopal, B., Reeves, M., Hopkins, M. \& Kruschwitz, N., (2009), "Sustainability and competitive advantage", MIT Sloan Management Review, Vol. 51, No. 1, pp. 18-27.

Björklund, M. (2005), Purchasing Practices of Environmentally Preferable Transport Services Guidance to increased shipper considerations, Doctoral Thesis, Department of Industrial Management and Logistics, Lund University.

Bourlakis, C. \& Bourlakis, M. (2005), "Information technology safeguards, logistics asset specificity and fourth-party logistics network creation in the food retail chain", Journal of Business \& Industrial Marketing, Vol. 20, No. 2, pp. 88-98.

Carbone, V. \& Stone, M.A. (2005) "Growth and relational strategies used by the European logistics service providers: Rationale and outcomes", Transportation Research Part E, Vol. 41, No. 6, pp. 495-510.

Charter, M. \& Clark, T. (2008), "Product sustainability: organisational consideration", International Journal of Product Development, Vol. 6, No. 3/4 , pp. 251-275. 
Clinton, S. (2008), "Importance of Technology Investments in the Logistics Service Providers: A Case Study of UPS and its use of online tools", Journal of Applied Business Research, Vol. 24, No. 2, pp. 67-80.

Coley, F. \& Lemon, M. (2009), "Exploring the design and perceived benefit of sustainable solutions: a review", Journal of Engineering Design, Vol. 20, No. 6, pp. 543-554.

CSCMP, (2011), Council of Supply Chain Management Professionals, CSCMP's Glossary, [Online], Available: http://cscmp.org/digital/glossary/glossary.asp>, Accessed on the $25^{\text {th }}$ of October 2011.

Cullinane, S. \& Edwards, J. (2010), Assessing the environmental impacts of freight transport, in McKinnon et al. (eds) Green Logistics. Improving the environmental sustainability of logistics, London: KoganPage, pp. 31-48.

Dahlman, F., Brammer, S. \& Millington, A. (2008), "Barriers to proactive environmental management in the United Kingdom", Journal of General Management, Vol. 33, No. 3, pp. 120 .

Daugherty, P.J., Sabath, R.E \& Rogers, D.S., (1992), "Competitive Advantage Through Customer Responsiveness", The Logistics and Transport Review, Vol. 28, No. 3, pp. 257-272.

DB Schenker (2011) Taking responsibility. Providing Solutions, Environmental Brochure 2011, Deutche Bahn AG.

DHL, (2010), Delivering Tomorrow - Towards Sustainable Logistics, [Online], Available: http://www.dpdhl.com/content/dam/logistik populaer/trends/StudieSustainableLogisti cs/study towards sustainable logistics.pdf, Accessed October 28 2011.

Denyer, D. \& Tranfield, D. (2009), "Producing a Systematic review" in Buchanan, D.A. \& Bryman, A. (2009), The SAGE Handbook of Organizational Research Methods, SAGE Publications Ltd, London, United Kingdom.

Dobie, K. (2005), “The core Shipper Concept: A Proactive Strategy for Motor Freight Carriers", Transportation Journal, Vol. 44, No. 2, pp. 37-53.

Drewes Nielsen, L., Homann Jespersen, P., Petersen, T. \& Gjesong Hansen, L. (2003) "Freight transport growth - a theoretical and methodological framework", European Journal of Operational Research, Vol. 144, No. 2, pp. 295-305.

Dubé, L. \& Paré, G., (2002), "Rigor in Information Systems Positivist Case Research: Current Practices Trends and Recommendations", MIS Quarterly, Vol. 27, No. 4, pp. 597635.

Eisenhardt, K., (1989), "Building theories from case study research", Academy of Management Review, Vol. 14, No. 4, pp. 532-550. 
Eisenhardt, K.M. \& Graebner, M.E. (2007), “Theory Building from Cases: Opportunities and Challenges", Academy of Management Journal, Vol. 50, No. 1, pp. 25-32.

Eltayeb, T.K., \& Zailani, S. (2009), "Going green through green supply chain initiatives towards environmental sustainability", Operations and Supply Chain Management, Vol. 2, No. 2, pp. 93-110.

ETTAR project (2007) "Transport and the environment: barriers to the take up of environmental technologies in the transport sector", summary report, 25-26 October, Gothenburg, Sweden.

Evangelista, P. (2004), "Leveraging technology capabilities in the 3PL Industry", Logistics Solutions, Vol. 7, No. 1, pp. 24-29.

Evangelista, P. (2011), ICT diffusion in SMEs - An investigation into the Italian transport and logistics service industry, Edizioni Scientifiche Italiane, Napoli, Italy.

Evangelista, P. \& Sweeney, E. (2006), "Technology usage in the supply chain: the case of small 3PLs", International Journal of Logistics Management, Vol. 17, No. 1, pp. 55-74.

Evangelista, P., Sweeney, E., Ferruzzi, G., \& Carrasco, J.C. (2010), “Green supply chain initiatives in transport and logistics service industry: an exploratory case study analysis", in proceedings of the Logistics Research Network Conference Towards the Sustainable Supply Chain: Balancing the needs of Business, Economy and the Environment, 8 ${ }^{\text {th }}-10^{\text {th }}$ September, Harrogate, UK, pp. 195-203.

Evans, S., Partidario, P. \& Lamberts, J. (2007), "Industrialization as a key element of sustainable product-service solutions", International Journal of Production Research, Vol. 45, No. 18-19, pp. 4225-4246.

Fabbe-Costas, N., Jahre, M. \& Roussat, C. (2009), "Supply chain integration the role of logistics service providers", International Journal of Productivity and Performance Management, Vol. 58, No. 1, pp. 71-91.

Fitzgerald, L. \& Dopson, S. (2009), "Comparative Case Study Designs: Their Utility and Development in Organizational Research" in Buchanan, D.A. \& Bryman, A. (2009), The SAGE Handbook of Organizational Research Methods, SAGE Publications Ltd, London, United Kingdom.

Flynn, B.B., Kakibara, S.S., Schroeder, R.G., Bates, K.A. \& Flynn, E.J. (1990), “Empirical Research Methods in Operations Management", Journal of Operations Management, Vol. 9, No. 2, pp. 250-284.

Forza, C. (2002), "Survey research in operations management: a process-based perspective", International Journal of Operations \& Production Management, Vol. 22, No. 2, pp. 152-194. 
Foster, S.T, Sampson, S.E \& Dunn, S.C., (2000), “The impact of customer contact on environmental initiatives for service firms", International Journal of Operations and Production Management, Vol. 20, No. 2, pp. 187-203.

Grant, D.B., Teller, C. \& Teller, W. (2005), "Web-based Surveys in Logistics Research: An Empirical Application" in Kotzab, H., Seuring, S., Müller, M. \& Reiner, G. (2005), Research Methodologies in Supply Chain Management, Physica-Verlag Heidelberg, Germany.

Handfield, R.S. \& Melnyk, S.A. (1998), "The scientific theory-building process: a primer using the case of TQM", Journal of Operations Management, Vol. 16, No. 4, pp. 321-329.

Henriques, I. \& Sadorsky, P. (1999). "The relationship between environmental commitment and managerial perceptions of stakeholder importance", Academy of Management Journal, Vol. 42, No.1, pp. 87-99.

Hertz, S. \& Alfredsson, M. (2003). "Strategic development of third part logistics providers”, Industrial Marketing Management, Vol. 32, No. 2, pp. 139-149.

Ho, Y.H., Lin C.Y. \& Chiang, S.H. (2009) "Organizational determinants of green innovation implementation in the logistics industry", The International Journal of Organizational Innovation, Vol. 2, No. 1, pp. 5-12.

Hong, P., Kwon, H.B., \& Roh, J.J., (2009), "Implementation of strategic green orientation in supply chain. An empirical study of manufacturing firms", European Journal of Innovation Management, Vol. 12, No. 4, pp. 512-532.

Juga, J., Juntunen, J. \& Grant, D.B., (2010), "Service quality and its relation to satisfaction and loyalty in Logistics outsourcing relationships", Managing Service Quality, Vol. 20, No. 6, pp. 496-510.

Kassinis, G.I. \& Soteriou, A.C., (2003), "Greening the Service Profit Chain: The Impact of Environmental Management Practices", Production \& Operations Management, Vol. 12, No. 3, pp. 386-402.

Kovács, G. \& Spens, K.M. (2005) “Abductive reasoning in logistics research”, International Journal of Physical Distribution and Logistics Management, Vol. 35, No. 2, pp. 132-144.

Lai, K-H., (2004), "Service capability and performance of logistics service providers", Transportation Research: Part E, Vol. 40, No. 5, pp. 385-399.

Lai, K-H., Ngai, E.W.T. \& Cheng, T.C.E. (2002), “Measures for evaluating supply chain performance in transport logistics", Transportation Research: Part E, Vol. 38, No. 6, pp. 439457.

Lambert, D.M. \& Harrington, T.C. (1990), "Measuring non-response bias in customer service mail surveys", Journal of Business Logistics, Vol. 11, No. 2, pp. 5-25. 
Langley, C.J., van Dort, E., Ang, A. \& Sykes, S.R. (2005), 2005 Third-Party Logistics Results and findings of the 10th Annual Study, Georgia Institute of Technology, Atlanta, USA

Larson, P. \& Gammelgaard, B. (2001), "Logistics in Denmark: A Survey of the Industry", International Journal of Logistics: Research and Applications, Vol. 4, No. 2, pp. 191-206.

Lemoine, O.W. \& Skjoett-Larsen, T. (2004) "Reconfiguration of supply chains and implications for transport", International Journal of Physical Distribution \& Logistics Management, Vol. 34, No. 10, pp. 793-810.

Lieb, R. \& Bentz, B.A. (2004) "The Use of Third-Party Logistics Services by Large American Manufactures: The 2003 Survey", Transportation Journal, Vol. 43, No. 3, pp. 2433.

Lieb, R. \& Bentz, B.A. (2005) "The Use of Third-Party Logistics Services by Large American Manufactures: The 2004 Survey", Transportation Journal, Vol. 44, No. 2, pp. 5-15.

Lieb, K.J. \& Lieb, R.C. (2010), "Environmental sustainability in the third-party logistics (3PL) industry", International Journal of Physical Distribution \& Logistics Management, Vol. 40, No. 7, pp. 524-533.

Lieb, R. \& Miller, J. (2002) "The Use of Third-party Logistics Services by Large US Manufactures, The 2000 Survey", International Journal of Logistics: Research \& Applications, Vol. 5, No. 1, pp. 1-12.

Lin, C.Y., \& Ho, Y.H (2008) “An empirical study on logistics service providers' intention to adopt green innovations", Journal of Technology Management \& Innovation, Vol. 3, No. 1, pp. 17-26.

Lu, L.Y.Y., Wu, C.H. \& Kuo, T-C., (2007), "Environmental principles applicable to green supplier evaluation by using multi-objective design analysis", International Journal of Production Research, Vol. 45, No. 18/19, pp. 4317-4331.

Maloni, M.J. \& Carter, C.R., (2006), “Opportunities for research in third-party logistics", Transportation Journal, Vol. 45, No. 2, pp.23-38.

Marasco, A. (2008), “Third-party logistics: A literature review", International Journal of Production Economics, Vol. 113, No. 1, pp. 127-147.

Marchant, C. (2010) "Reducing the environmental impact of warehousing", in McKinnon et al. (eds) (2010) Green Logistics: Improving the environmental sustainability of logistics, Kogan Page, pp. 167-192.

Martinsen U., \& Huge-Brodin M., (2010) "Greening the offerings of logistics service providers", in proceedings of the 22th Annual NOFOMA conference Logistics and Supply Chain Management in a Globalised Economy, University of Southern Denmark, pp. 969-984. 
Maxwell, D., Sheate, W. \& van der Vorst, R. (2006), "Functional and systems aspects of the sustainable product and service development approach for industry", Journal of Cleaner Production, Vol. 14, No. 17, pp. 1466-1479.

McGinnis, M.A. \& Kohn, J.W. (2002) "Logistics Strategy-Revisited", Journal of Business Logistics, Vol. 23, No. 2, pp. 1-17.

McKinnon, A., (2006), "A review of European truck tolling schemes and assessment of their possible impact on logistics systems", International Journal of Logistics: Research and Applications, Vol. 9, No. 3, pp.191-205.

McKinnon, A.C. (2010) Environmental sustainability: a new priority for logistics managers, in McKinnon et al. (eds) Green Logistics. Improving the environmental sustainability of logistics, London: KoganPage, pp. 3-30.

Mentzer, J.T., Flint, D.J. \& Hult, G.T.M. (2001) "Logsitics Service Quality as a SegmentCustomized Process”, Journal of Marketing, Vol. 65, No. 4, pp. 82-104.

Meredith, J. (1998), "Building operations management theory through case and field research", Journal of Operations Management, Vol. 16, No. 4, pp. 441-454.

Merriam-Webster Encyclopaedia, (2011), Merriam-Webster - an Encyclopaedia Britannica Company, Dictionary, [Online], Available: http://www.merriamwebster.com/dictionary, Accessed on the $25^{\text {th }}$ of October 2011.

Miles, M. and Hubermann, A., (1994), Qualitative Data Analysis: An Expanded Sourcebook, $2^{\text {nd }}$ ed. Sage Publications, Thousand Oaks, CA.

Mortensen, O. \& Lemonie, O. (2007), "Integration between manufactures and third party logistics provider?”, International Journal of Operations \& Production Management, Vol. 28, No. 4, pp. 331-359.

Murphy, P.R., \& Daley, J.M. (2001) "Profiling international freight forwarders: an update", International Journal of Physical Distribution and Logistics Management, Vol. 31, No. 3, pp. 152-168.

Murphy, P.R. \& Poist, R.F. (2000) "Third-Party Logistics: Some User Versus Providers Perspective", Journal of Business Logistics, Vol. 21, No. 1, pp. 121-133.

Panayides, P.M. \& So, M. (2005), "Logistics service provider-client relationships", Transportation Research: Part E, Vol. 41, No. 3, pp. 179-200.

Persson, G. \& Virum, H. (2001) "Growth Strategies for Logistics Service Providers: A Case Study", The International Journal of Logistics Management, Vol. 12, No. 1, pp. 53-64.

Piecyk, M.I., (2010a), Analysis of Long-term Freight Transport, Logistics and Related $\mathrm{CO}_{2}$ Trends on a Business-as-Usual Basis, Doctoral Thesis, Heriot-Watt University, Edinburgh, UK. 
Piecyk, M. (2010b), Carbon auditing of companies, supply chains and products, in McKinnon et al. (eds) Green Logistics. Improving the environmental sustainability of logistics, London: KoganPage, pp. 49-67.

Porter, M.E. \& van der Linde, C. (1995), "Green and Competitive", Harvard Business Review, Vol. 73, No. 5, pp. 120-134.

Post, J. \& Altman, B. (1994), "Managing the Environmental Change Process: Barriers and Opportunities”, Journal of Organizational Change Management, Vol. 7, No. 4, pp. 64-81.

Pujari, D., Wright, G. \& Peattie, K. (2003), "Influences on environmental new product development performance”, Journal of Business Research, Vol. 56, No. 8, pp. 657-671.

Rajesh, R., Pugazhendhi, S., Ganesh, K., Muralidharan, C. \& Sathiamonnrthy, R. (2011), "Influence of 3PL service offerings on client performance in India", Transportation Research: Part E, Vol. 47, No. 2, pp. 149-165.

Rao, P. \& Holt D. (2005) "Do green supply chains lead to economic performance?," International Journal of Operations and Production Management, Vol. 25, No. 9, pp. 898-916.

Roth, A. \& Kåberger, T. (2002), "Making transport systems sustainable”, Journal of Cleaner Production, Vol. 10, No. 4, pp. 361-371.

Rothengatter, W. (2010), "Climate change and the contribution of transport: Basic facts and the role of aviation", Transportation Research Part D, Vol. 15, No. 1, pp. 5-13.

Salomone, R., (2008), "Integrated management systems: experience in Italian organisations", Journal of Cleaner Production, Vol. 16, No. 16, pp. 1786-1806.

Sarkis, J. (ed.) (2006) Greening the Supply Chain, Springer, London.

Seidel, M., Seidel, R., Tedford, D., Cross, R., Wait, L. \& Hämmerle, E. (2009), "Overcoming Barriers to Implementing Environmentally Benign Manufacturing Practices: Strategic Tools for SMEs”, Environmental Quality Management, Vol. 18, No. 3, pp. $37-55$.

Selviaridis, K., \& Spring, M., (2007), “Third party logistic: a literature review and research agenda", The International Journal of Logistics Management, Vol. 18, No. 1, pp.125-150.

Shi, H., Peng, S.Z., Liu, Y. \& Zhong, P. (2008), "Barriers to the implementation of cleaner production in Chinese SMEs: government, industry and expert stakeholders' perspective", Journal of Cleaner Production, Vol. 16, No. 7, pp. 842-852.

Siggelkow, N. (2007), "Persuasion with case studies", Academy of Management Journal, Vol. 50, No. 1, pp. 20-24. 
Skjoett-Larsen, T. (2000), "European logistics beyond 2000", International Journal of Physical Distribution and Logistics Management, Vol. 30, No. 5, pp. 377-387.

Spens, K.M. \& Bask, A.H. (2002), "Developing a Framework for Supply Chain Management”, International Journal of Logistics Management, Vol. 13, No. 1, pp. 73-88.

Srivastava, S.K. (2007), "Green supply-chain management: a state-of-the-art literature review”, International Journal of Management Reviews, Vol. 9, No. 1, pp. 53-80.

Stank, T.P. \& Goldsby, T.J. (2000) "A framework for transportation decision making in an integrated supply chain”, Supply Chain Management: An International Journal, Vol. 5, No. 2, pp. $71-77$.

Stock, J.R., (1997). “Applying theories from other disciplines to logistics", International Journal of Physical Distribution \& Logistics Management, Vol. 27, No. 9/10, pp. 515-539.

Stuart, I., McCutcheon, D., Handfield, R., McLachlin, R. \& Samson, D. (2002) "Effective case research in operations management: a process perspective", Journal of Operations Management, Vol. 20, No. 5, pp. 419-433.

Sweeney, E. \& Evangelista, P. (2005) "3PL definition and taxonomy", Logistics Solutions, Vol. 7, No. 2, pp. 9-10.

Tranfield, D., Denyer, D. \& Smart, P., (2003), “Towards a Methodology for Developing Evidence-Informed Management Knowledge by Means of Systematic Review", British Journal of Management, Vol. 14, No. 3, pp. 207-222.

Trafikverket, (2010), Trafikslagsövergripande planeringsunderlag för begränsad klimatpåverkan, [Online], Available:http://www.trafikverket.se/Privat/Miljo-och-halsa/Klimat/Vad-gorTrafikverket/, Accessed October 28th 2011.

Transportstyrelsen, (2011), Transportstyrelsens handlingsplan för åtgärder $i$ miljömålsystemet, [Online],Available:http://www.transportstyrelsen.se/Global/Om_oss/Miljo/Faststalld_h andlingsplan atgarder miliomalssystemet v1 0.pdf, Accessed October $26^{\text {th }} 2011$.

Vachon, S. \& Klassen, R.D., (2006), "Extending green practices across the supply chain The impact of upstream and downstream integration", International Journal of Operations \& Production Management, Vol. 26, No. 7, pp. 795-821.

van Hemel, C. \& Cramer, J. (2002), "Barriers and stimuli for ecodesign in SMEs", Journal of Cleaner Production, Vol. 10, No. 5, pp. 439-453.

van Hoek, R.I., (1999), "From Reversed Logistics to Green Supply Chains", Supply Chain Management, Vol. 4, No. 3, pp. 129-134. 
van Hoek, R. \& Chong, I. (2001), "Epilogue: UPS Logistics-practical approaches to the esupply chain", International Journal of Physical Distribution \& Logistics Management, Vol. 31, No. 6, pp. 463-468.

van Hoek, R.I. \& Johnson, M., (2010), "Sustainability and energy efficiency. Research implication from an academic roundtable and two case examples", International Journal of Physical Distribution \& Logistics Management, Vol. 40, No. 1/2, pp.148-158.

Waage, S., Geiser, K., Irwin, F., et al. (2005), "Fitting together the building blocks for sustainability: a revised model for integrating ecological, social, and financial factors into business decision-making", Journal of Cleaner Production, Vol. 13, No. 12, pp. 1145-1163.

Walker, H., Di Sisto, L. \& McBain, D., (2008), "Drivers and barriers to environmental supply chain management practices: Lessons from the public and private sectors", Journal of Purchasing \& Supply Management, Vol. 14, No. 1, pp. 69-85.

Wolf, C. \& Seuring, S. (2010). "Environmental impacts as buying criteria for third party logistical services", International Journal of Physical Distribution \& Logistics Management, Vol. 40, No. 1, pp. 84-102.

Wong, V., Turner, W. \& Stoneman, P. (1996), "Marketing Strategies and Market Prospects for Environmentally-Friendly Consumer Products", British Journal of Management, Vol. 7, No. 3, pp. 263-281.

Woodburn, A. \& Whiteing, A. (2010) in McKinnon et al. (eds) Green Logistics. Improving the environmental sustainability of logistics, London: KoganPage, pp. 124-139.

Wu, H.J. \& Dunn, S.C., (1995), "Environmentally responsible logistics systems", International Journal of Physical Distribution \& Logistics Management, Vol. 25, No. 2, pp.20-38.

WWF, (2009a), World Wildlife Foundation (WWF), G8 Climate Scorecards, [Online], Available:http://www.wwf.se/source.php/1253675/G8\%20Climate $\% 20$ Scorecards $\% 202$ 009.pdf. Accessed on the $26^{\text {th }}$ of October 2011.

WWF, (2009b), World Wildlife Foundation (WWF), Climate Scorecards - Sweden, [Online], Available:http://www.wwf.se/source.php/1253677/G8\%20Climate $\% 20$ Scorecards $\% 202$ 009 Sweden.pdf, Accessed on the 26 th of October 2011.

Yin, R.K. (2009), Case Study Research - Design and Methods, 4th edition, SAGE, Thousand Oaks, USA.

Yu, T., Ellinger, A. E. \& Haozhe, C. (2010), "Third-party logistics provider customer orientation and customer firm logistics improvement in China", International Journal of Physical Distribution \& Logistics Management, Vol. 40, No. 5, pp. 356-376.

Zacharia, Z.G. \& Mentzer, J.T. (2007) "The role of logistics in new product development", Journal of Business Logistics, Vol. 28, No. 1, pp. 83-110. 
Zhu, Q. \& Sarkis, J. (2004) "Relationships between operational practices and performance among early adopters of green supply chain management practices in Chinese manufacturing enterprises". Journal of Operations Management, Vol. 22, No. 3, pp. 265-289.

Zhu, Q. \& Sarkis, J. (2006) “An intersectional comparison of green supply chain management in China: drivers and practices", Journal of Cleaner Production, Vol. 14, No. 5, pp. 472-486. 
APPENDIX 1

INTERVIEW GUIDE 



\section{QUESTIONS TO THE LOGISTICS SERVICE PROVIDERS}

\section{The Respondent}

- What is your position at the company?

\section{The company in general}

- Range of service offerings (i.e. different kinds of transport modes, consulting, valueadded services)

- The company's turnover? (on the Swedish market)

- The number of employees in Sweden?

- What is the geographical range of your company's operations?

- What proportion of the turnover is based on consumer market customers? (percentage)

\section{The company with regard to green initiatives}

- What kind of green related service offerings does your company offer?

- How many full-time jobs within your company are dedicated to the environmental work (in Sweden)?

\section{Drivers - internal}

- Please indicate and comment, into which extent the following internal drivers influence your company when addressing environmental issues $(1=$ not at all, $5=$ very much $)$

$\circ$ Cost savings

$\begin{array}{lllll}1 & 2 & 3 & 4 & 5\end{array}$

o Potential source of revenue or cash flow

$\begin{array}{lllll}1 & 2 & 3 & 4 & 5\end{array}$

○ Employees involvement

$\begin{array}{lllll}1 & 2 & 3 & 4 & 5\end{array}$

- The ability to be an interesting long-term innovation opportunity (long-termed strategy)

$\begin{array}{lllll}1 & 2 & 3 & 4 & 5\end{array}$

o It's the top of the business agenda (short-termed strategy)

$\begin{array}{lllll}1 & 2 & 3 & 4 & 5\end{array}$

- Other internal drivers that influence your company when addressing environmental issues?

\section{Drivers - external}

- Please indicate and comment, into which extent the following external drivers influence your company when addressing environmental issues $(1=$ not at all, $5=$ very much)

o Government legislation

1

○ Customers

1

- Competitors
2

2

2
3

3

3
4

4

4
5

5 
○ Society

1

2

3

4

5

○ Suppliers

1

2

3

4

5

o Investors/owners

1

2

3

4

5

- Other external drivers that influence your company when addressing environmental issues?

\section{Barriers - internal}

- Please indicate and comment, into which extent the following internal barriers influence your company when addressing environmental issues ( $1=$ not at all, $5=$ very much)

- Financial and economic barriers
1
2
3
4
5

- Technical barriers

$\begin{array}{lllll}1 & 2 & 3 & 4 & 5\end{array}$

- Information barriers

$\begin{array}{lllll}1 & 2 & 3 & 4 & 5\end{array}$

$\circ$ Managerial barriers

$\begin{array}{lllll}1 & 2 & 3 & 4 & 5\end{array}$

- Organizational barriers

$\begin{array}{lllll}1 & 2 & 3 & 4 & 5\end{array}$

- Other internal barriers that influencing your company when addressing environmental issues?

\section{Barriers - external}

- Please indicate and comment, into which extent the following external barriers influence your company when addressing environmental issues ( $1=$ not at all, $5=$ very much $)$

○ Policy barriers

$\begin{array}{llllll}1 & 2 & 3 & 4 & 5\end{array}$

○ Market barriers

$\begin{array}{lllll}1 & 2 & 3 & 4 & 5\end{array}$

- Other external barriers that influencing your company when addressing environmental issues?

\section{Pricing green logistics service offerings}

- In what way does your company work with different price models (taking the environmental issues into account) towards your customers?

$\circ$ Pros and cons?

- Why does your company use a certain type of price model (or not)? 
- By working in this way, what are the potentials in the future?

- In what way would your company like to work with green offerings in the future? 

APPENDIX 2

CASE REPORT 



\title{
CASE COMPANY PRESENTATIONS
}

This case report introduces and presents the six different logistics service providers (LSPs) studied in this licentiate thesis. In order to encourage openness of response during the data gathering process, it was agreed that company names would remain anonymous, and the case companies were therefore labelled Alfa, Beta, Gamma, Delta, Epsilon and Zeta.

The information in this report has mainly been obtained from telephone interviews with one representative from each company. Each interview lasted for at least one hour, and in some cases, further contact with the company's representative was obtained from company visits, by telephone and by email. Besides the interviews, secondary data such as information about the companies has been collected from a variety of information sources, including company reports and websites.

Below, a general overview of each participating company is provided. In addition, different drivers and barriers affecting the adoption of green initiatives within the company are described. The companies' green service offerings as well as how green issues can be handled and integrated into the price picture are also discussed.

In order to make it easier for the reader, the following list of drivers and barriers in Table 1 have been studied within each of the participating LSPs. The respondents were asked to specify the impact of each of factor on a scale of 1 to 5 (where 5 had the greatest impact), later translated into a scale with a range of low (1-2), medium (3) and high (4-5).

Table 1 Studied drivers and barriers in the case study.

\begin{tabular}{|l|l|l|}
\hline \multirow{4}{*}{ Internal } & Drivers & Barriers \\
\cline { 2 - 3 } & Cost savings & Financial and economic \\
\cline { 2 - 3 } & Potential source of revenue & Technical \\
\cline { 2 - 3 } & Employee involvement & Information \\
\cline { 2 - 3 } & Long-term innovation opportunity & Managerial \\
\cline { 2 - 3 } External & The top of the business agenda & Organizational \\
\cline { 2 - 3 } & Government legislation & Policy \\
\cline { 2 - 3 } & Customers & Market \\
\cline { 2 - 3 } & Sompetition & \\
\cline { 2 - 3 } & Society & \\
\cline { 2 - 3 } & Suppliers & \\
\cline { 2 - 3 } & Investor/owner & \\
\hline
\end{tabular}

\begin{abstract}
ALFA
Alfa is one of the largest transportation intermediation companies in Sweden. The company had an approximate turnover of 61.6 millions EURO during 2009 and has about 111 employees of whom two work full-time with environmental sustainable initiatives. Alfa has about 140 subcontracted carriers, who also are part owners in the company. This results in a total of more than 500 people employed in the entire concern.

The activities within the company are divided into three business areas; Building \& Construction, Long-distance \& Distribution and Transport Tank \& Environment. The geographical reach is mainly local and regional.
\end{abstract}




\section{Service offerings and customers}

The major task of Alfa is to offer transportation intermediations. This includes sales in three business areas (which are mentioned above);

- Building \& Construction; this business area includes machinery and vehicles for all types of contract works such as ground works, shift and transport operations.

- Long-distance \& Distribution; includes major vehicles, such as cars with trailers for the transportation of bulk cargo, paper, agricultural transport and cement.

- Tank \& Environment; consist of two subareas Sludge \& Flush and Waste \& Recycling. Sludge \& Flush includes tank cleaning, the inspection of oil tanks and the transport of hazardous liquid waste and residues from various process industries and other industries. Waste \& Recycling is a fast growing area and includes the transport and storage of hazardous waste.

Over $90 \%$ of Alfa's services cover the full range of the transport market. However, the company does not offer refrigerated transports. Added services are offered to all transport service offerings as a complement, depending on the demand. For example, in the business area Long-distance \& distribution, the company offers short- and long-term storage as an additional service. Their customers range from industries and municipalities to end-users.

\section{Drivers for the adoption of green initiatives}

Table 2 illustrates Alfa's perception of the impact of each of the internal and external drivers mentioned above, followed by additional comments regarding each of these drivers.

Table 2 Studied drivers affecting Alfa's adoption of green initiatives.

\begin{tabular}{|c|c|c|}
\hline & Drivers & Impact \\
\hline \multirow[t]{5}{*}{ Internal } & Cost savings & Medium \\
\hline & Potential source of revenue & High \\
\hline & Employee involvement & High \\
\hline & Long-term innovation opportunity & High \\
\hline & The top of the business agenda & High \\
\hline \multirow[t]{6}{*}{ External } & Government legislation & High \\
\hline & Customers & High \\
\hline & Competition & Medium \\
\hline & Society & High \\
\hline & Suppliers & Medium \\
\hline & Investor/owner & Medium \\
\hline
\end{tabular}

\section{Internal drivers}

Cost savings; customers today are not willing to pay for environmental aspects of the transport. There is no profitability in a short-term perspective, and instead customers choose alternatives with lower prices. Hence, there are customers that take environmental issues into account but do not want to pay extra for these. Potential source of revenue; better environmental sustainable work may lead to new customers and market shares, but monitoring the money flow connected to environmental aspects today is weak and difficult to handle. Employee involvement; this factor has increased over time. Employees have begun to realize the importance of environmental work and also that it could 
provide some future benefits. Environmental issues have become a matter of public interest and therefore there is a reason to become involved in this work. The ability to be an interesting long-term innovation opportunity and the top of the business agenda; Alfa believes that it is not realistic within the transportation sector to view environmental aspects and develop strategies and goals only from a short-term perspective. Alfa has both short- and longterm goals when it comes to its adoption of green initiatives. The time periods for the short-termed goals are at least one year, and the goals are monitored annually and the results of the work are publicly reported.

\section{Other internal drivers}

Alfa mentioned a powerful and driven board, who are interested in environmental issues and can see their importance, as another prominent internal driver. This could provide good support for implementing changes linked to the adoption of green initiatives due to the fact that the changes have a greater impact and are more likely to be received with trust and confidence by employees. It is also easier to show employees that working for the environment is not only good for the company but is also advantageous from a global perspective.

\section{External drivers}

Government legislation; there are some environmental demands from local authorities, and if these demands are not followed, Alfa risks not getting these kinds of jobs. However, the specifications of the requirements are quite often rather vague and complicated. This in turn leads to problems in understanding what these requirements actually mean. Furthermore, Alfa does not feel that these environmental requirements are followed up in a proper manner. Customers; business customers have more reasonable green demand than country councils. These demands are more business oriented and logical. Alfa perceives that customers are interested in and aware of the importance of environmental questions, but are not willing to pay for it. Competition; it is not a major driver, although they do engage in some market research exist in order to benchmark how their competitors operate with green initiatives. This is perceived as a good source of inspiration. Society; the brand is important in order to win new customers. The company is perhaps not mentioned in the media as much as their competitors, but Alfa chooses to focus on their performance rather than on marketing. Suppliers; are perceived as not taking any green initiatives due to the fact that these initiatives are associated with costs, which suppliers are not willing to pay for. Investors/owners; pressure and questions from investors and owners have recently increased, even though Alfa wishes they could be even more active.

\section{Other external drivers}

Alfa highlights networking with other companies and organisations in order to get inputs and ideas which thereafter could be implemented in its own company as another.

\section{Barriers for the adoption of green initiatives}

Alfa's perception of the impact of the barriers studied is illustrated in Table 3, followed by additional comments on each of the internal and external barriers studied. 
Table 3 Studied barriers affecting Alfa's adoption of green initiatives.

\begin{tabular}{|l|l|l|}
\hline \multirow{3}{*}{ Internal } & Barriers & Impact \\
\cline { 2 - 3 } & Financial and economic & High \\
\cline { 2 - 3 } & Technical & Medium \\
\cline { 2 - 3 } & Information & Low \\
\cline { 2 - 3 } & Managerial & Low \\
\cline { 2 - 3 } & Organizational & Low \\
\hline \multirow{2}{*}{ External } & Policy & High \\
\cline { 2 - 3 } & Market & High \\
\hline
\end{tabular}

\section{Internal barriers}

Financial and economic; Alfa states that all green solutions are much more expensive than others, and financial issues are thus considered as a major barrier. Technical; there are some existing solutions which take green considerations into account, but not all of these solutions have been implemented or tested enough. This in turn can lead to difficulties when it comes to justifying these kinds of investments within the company. Information is not seen as a major barrier since Alfa states that the company has well-developed ITsystems that are used both within the own company and with their customers. Instead, the problems are more related to selecting the right kind of information. Managerial; this is not perceived as a barrier. Alfa states that it has good support from the board. Organizational; this factor is not perceived as a barrier either, but Alfa stress that there must also be a certain acceptance regarding the fact that there will always be opposition to change and reluctance to accept them.

\section{External barriers}

Policy is perceived to be a major barrier since Alfa feels the pressure to invest in something even though Alfa is against it. Hence legal requirements must be followed even if the company has no resources for meeting these requirements. Market is a significant barrier. For example, the transport industry has been negatively labelled as the largest emitter of $\mathrm{CO}_{2}$, which Alfa perceives as a barrier and stresses the difficulties of washing this label off. Furthermore, Alfa highlights that green investments could be less difficult to justify if customers were more willing to pay for green initiatives. Alfa says that today these kinds of investments are made purely out of good will or as a result of a company's own driving force but without any financial gains.

\section{Other barriers}

Technical development is perceived as a barrier, especially when it comes to development and the research within the automotive sector.

\section{Green service offering and its pricing}

Alfa does not offer any concrete green service offering but instead, this is more or less integrated into different kinds of service offerings. For example, when Alfa offers consultation in projects, the company is also able to offer follow-ups regarding environmental impacts. The company also offers eco-driving instructions to the drivers if customers demand it. For each transport service offering there is an alternative option that is more environmental friendly. The more environmental friendly option may consist of better engines, alternative fuels or specific adjustments to the vehicle. Alfa is also able to offer its customers $\mathrm{CO}_{2}$ declarations on their transport services. Alfa states that the 
number of times they initiative the introduction of green service offerings to customers compared with those times when customers first take the initiative are more or less equal.

Alfa has no pricing model that takes sustainability concerns into account. Service offerings, which include more environmentally friendly vehicles, are priced in a similar way as for a non-environmentally friendly vehicle. The price of $\mathrm{CO}_{2}$ declarations is often decided in agreement with customers and in some cases is included in the regular price. Usually Alfa offers these kinds of services free of charge in order to increase the value of the service offering. However, if customers require a more regular reporting, for example every week, an additional cost for this service would be discussed. Alfa is not entirely against having a more value-based pricing model because the company wants to have some kind of return on the resources used. Hence, the company does not believe that in the near future customers will pay extra for a green service offering. Therefore, Alfa has decided not to charge for these service offerings but instead, these are added as an extra value to existing service offerings. The representative of Alfa states:

"The problem is that there are very few customers who value and put sustainability at such a high level to even figure out a special price for these kind of service offerings."

\section{BETA}

Beta is one of the largest LSPs in the northern part of Sweden. The turnover in 2009 was approximately 124.8 millions euro and today there are 94 employees. Three of these employees work full-time with quality, environment and education, and act as a supporting function to the different business areas. This function deals with continuous improvements in the form of certificates, networking with authorities and participations of different conferences. This is done in order to get inspiration for the company's own operations. Beta has about 260 subcontracted carriers, who also are part owners in the company, which lead to that the total number of employees is about 1000 people in the entire concern.

The company is divided into five business areas: Facility and Contract, Thermo, Industry, Wood and Chip and Special. Beta handles everything from gravel and industrial goods to sensitive consumer products and temperature-sensitive foods. The geographical area is mainly the northern part of Sweden.

\section{Service offerings and customers}

A description of the different business areas follows below;

- Facility and Contract; includes services related to the construction and maintenance of roads, as well as the transportation of materials to building sites. This business area targets customers in the contract-, industrial- and construction-areas.

- Thermo; consists of refrigerated transports of food from wholesalers to stores, schools, kindergartens and nursing homes. 
- Industry; offers the efficient handling of materials, goods and materials that secure the entire logistics flow. This business area also provides handling for inventory management.

- Wood and Chips; the main target is the wood-processing industry and Beta transports wood and chips that are used later to bio fuel.

- Special; includes all transport operations with very demanding needs such as extremely long, high, wide or heavy freights. Beta also works with other carriers in order to cover sea, air and rail transport.

The operations of Beta mainly concern full-load goods, and the company's main target is businesses. Furthermore, Beta works in close cooperation with its customers in order to develop complete logistics solutions.

\section{Drivers for the adoption of green initiatives}

The impact of the drivers studied and the ways in which these affect Beta's adoption of green initiatives are illustrated and describe below.

Table 4 Studied drivers affecting Beta's adoption of green initiatives.

\begin{tabular}{|l|l|l|}
\hline \multirow{4}{*}{ Internal } & Drivers & Impact \\
\cline { 2 - 3 } & Post savings & High \\
\cline { 2 - 3 } & Employee involvement & Medium \\
\cline { 2 - 3 } & Long-term innovation opportunity & High \\
\cline { 2 - 3 } & The top of the business agenda & High \\
\hline \multirow{3}{*}{ External } & Government legislation & High \\
\cline { 2 - 3 } & Customers & High \\
\cline { 2 - 3 } & Competition & High \\
\cline { 2 - 3 } & Society & Low \\
\cline { 2 - 3 } & Suppliers & High \\
\cline { 2 - 3 } & Investor/owner & Medium \\
\hline
\end{tabular}

\section{Internal drivers}

Cost savings is perceived as a major challenge within the company. Beta works a lot to improve logistics activities and strives to reduce costs for its subcontracted carriers, since it is the carriers that make the greatest environmental effort by reducing their fuel consumptions. Potential source of revenue; the company has clearly increased its turnover in recent years. This may not have been a direct result of the adoption of green initiatives, but Beta is convinced that it has been a contributing factor. The company would probably not have been where it is today without its efforts to improve the work with green initiatives as well as its investments in training and education and attempts to enhance skills both within the organisation and also among its subcontracted carriers. Employee involvement; Beta invests heavily in this driver in order to involve employees and increase the knowledge level regarding green thinking within the organisation. The company characterizes itself in general as a more goal-oriented organization rather than top-down. The significant impact of this driver for green initiatives can be demonstrated by the following quotation by the Beta representative: 


\begin{abstract}
"We work a lot to increase personal environmental responsibility and willingness to contribute to the whole company. We think this is a winning concept."
\end{abstract}

Beta wants not only to establish a working climate where each business unit should feel responsibility and independence in order to solve various problems that occur regarding green initiatives but also transfer this approach to the company's subcontracted carriers. Long-term innovation opportunity; Beta invests a lot of money and work in this driver. Beta stresses that it is important to show engagement and involvement in the green field, and that this may lead to an opportunity to be responsible for further development within this field. The top of the business agenda; this driver is seen as important since it is related to business with the customers. Beta stresses that the company possesses a lot of knowledge that can be used to help customers to solve various problems, as well as to inform them what it is possible or not possible to do regarding green initiatives. By providing its customers with this information, unreasonable demands can be decreased, even if green requirements from customers have become more realistic nowadays.

\title{
Other internal drivers
}

Beta mentions another driver, namely education. The company continuously offers employees education in sustainability in order to increase the enthusiasm among its employees.

\section{External drivers}

Government legislation; this driver is always existed and must of course be followed. However, Beta feels that they had already done a lot in this field even before it became a legal requirement. Customers are seen as very important drivers. The specifications of green requirements from customers have become more realistic and Beta works a lot in partnerships with its customers and discusses what is most appropriate from a sustainable perspective. Regarding the specifications of green requirements, Beta can distinguish certain differences in customers from different sectors. For example, customers from food industries have more detailed green requirements than customers from the industry sector. A possible explanation to this could be that the former are closer to the end consumers. Competition; Beta is aware of and follows its competitors' green development but this does not have a great impact on the company. Instead Beta concentrates on its own internal drivers. Society; Beta is very concerned about how the public views its business and has for example a website where it is possible to leave comments on how Beta conducts its activities. Suppliers, which in this case can be related to Beta's subcontracted carriers are not perceived as a main driver instead all initiatives comes from Beta. This can be explained by the fact that Beat is the one who handles the contracts and possesses all knowledge required from customers. Hence, Beta tries to increase the skills of the carriers by offering various training programmes, which for example focus on customers and eco driving. This has been a successful concept. Beta has noticed a more positive attitude towards these programmes and has also noticed a reduction in fuel 
consumption over the years, which can be explained by the training in eco driving. Investor/owner; the company has a committed board that focuses on sustainability issues. In addition, a new business plan is being developed which will include sustainability goals.

\section{Barriers for the adoption of green initiatives}

Table 5 shows the impact of the barriers studied, and how these influence Beta's adoption of green initiatives is described below.

Table 5 Studied barriers affecting Beta's adoption of green initiatives.

\begin{tabular}{|l|l|l|}
\hline & Barriers & Impact \\
\hline Internal & Financial and economic & High \\
\cline { 2 - 3 } & Technical & High \\
\cline { 2 - 3 } & Information & Medium \\
\cline { 2 - 3 } & Managerial & Low \\
\cline { 2 - 3 } & Organizational & High \\
\hline \multirow{2}{*}{ External } & Policy & Medium \\
\cline { 2 - 3 } & Market & High \\
\hline
\end{tabular}

\section{Internal barriers}

Financial; the company is constrained by the limited supply of environmental friendly vehicles on the market. Beta has a special agreement with the local authority who supports the company with the financially part. Without this support, such green investments could not been made. Beta must in someway get a return on this because it is so much more expensive to invest in these kinds of vehicles. Another barrier is the lack of fuel for such vehicles. Technical issues; are perceived as a big barrier. It is difficult to find alternative technical solutions to improve the sustainability part of the business. Information; Beta stresses that communication with its employees and customers has improved. Furthermore, the company has also created an IT-system, which has gradually been improved. Managerial issues; are not perceived as a barrier. Instead, the top management agreed on and supported the fact that Beta started to adopt green initiatives. Organizational barriers can have a significant impact if the entire organisation with all its subcontracted carriers is taken into account. Beta highlights the importance of everyone in the company understanding the purpose and benefits of adopting green initiatives.

\section{External barriers}

Policy; Beta argues that politicians have supported the wrong things and these have not shown any results. One example is the initiative with ethanol, and when it comes to heavy vehicles there are no alternatives to fuel these. Market; many customers have an environmental and quality department that generate green requirements which are later handed over to the purchasing department. Beta stresses that separate departments, which do not communicate leads to misunderstandings and the risk that some green aspects are never discussed. Furthermore, this also leads to unreasonable requirements difficult to achieve. This is a problem that affects many companies. Instead, Beta tries to integrate green awareness and thinking into every business unit but it takes time. The goal is that the business units by themselves could manage certain green requirements from customers. 


\section{Green service offering and its pricing}

Beta does not offer any concrete green service offerings, but instead tries to work sustainable into its businesses and develop partnerships with their customers. The company works with the innovation and development of its transports and tries to integrate those ecological aspects which exist naturally in sustainable and green thinking. However, Beta also tries to integrate economic and social aspects as well. Employees must have a willingness to contribute, but Beta also wants its customers to contribute to the company. Major decisions are made in consultation with customers in which both parties agree on the green requirements to be applied, for example how to measure environmental impact in order to give customers the information they need. The customers which Beta does not have partnerships with, are also perceived to have green requirements, but in the end, it all comes down to the price factor. However, the situation is a little bit different with customers with whom Beta has developed a partnership. Together with these customers Beta has focus on finding a long-term environmental solution that is suitable for both parties. The solutions identified should fit both the value-systems of Beta and of its customer.

Beta does not have any special pricing model that takes green considerations into account, instead these are viewed as an added value to offer its customers to increase the value chain and improve cooperation with its customers. This is a win-win relationship and the company hopes this will lead to potential new customers.

Beta measures $\mathrm{CO}_{2}$ emissions from its vehicles every year and is able to check the fuel consumptions of each vehicle in its system. Recently, it has become quite common that customers want a figure for the $\mathrm{CO}_{2}$ emissions consumed during a transport mission. However, the company has some problems with the system which supports these emissions calculations. In the future, customers may be able to get the emissions data from the system themselves, and Beta hopes that this will be possible in a few years.

Customers without any long contracts have to pay extra for $\mathrm{CO}_{2}$ reporting for example, since it takes some time to compile these reports. In the case of larger customers, this service offering is already included in the price and is not classified as an additional cost as long as the customer does not change their requirements during the project. However, in the future it is possible that Beta will have to charge for these service offerings. The most likely scenario however is that these service offerings will be included in the price for customers with longer contracts and customers without any fixed contracts will probably have to pay extra for these service offerings. The way in which Beta looks at the adoption of green initiatives within its own company can be summarised by the following remark by the Beta representative: 
"We want to have close cooperation with our customers, to be engaged, creative and solution-oriented and try new approaches and methodologies. We want to educate our employees so they can support our customers and everyone in the organisation should know where we are standing when it comes to sustainability issues. "

\section{GAMMA}

Gamma is a Nordic company that offers door-to-door express deliveries. The company is a franchise-based ground transportation organisation with access to 700 vehicles and to an extensive air route network. The turnover was approximately 28.8 millions EURO 2009. Gamma employs 85 people and approximately a half of one full-time job is connected to sustainability work.

\section{Service offerings and customers}

Gamma offers door-to-door express deliveries, throughout the Nordic region. The company provides systemised and customer-specific door-to-door express delivery solutions for time critical and high value products within high tech, automotive spare parts and medical equipment. Packages are transported either by road or air depending on the destination, size and urgency. The main group of customers consists of businesses and express deliveries to private customers occurs only rarely.

\section{Drivers for the adoption of green initiatives}

This section describes how the drivers studied affect Gamma's adoption of green initiatives and the impacts of these studied drivers are illustrated in Table 6 , below.

Table 6 Studied drivers affecting Gamma's adoption of green initiatives.

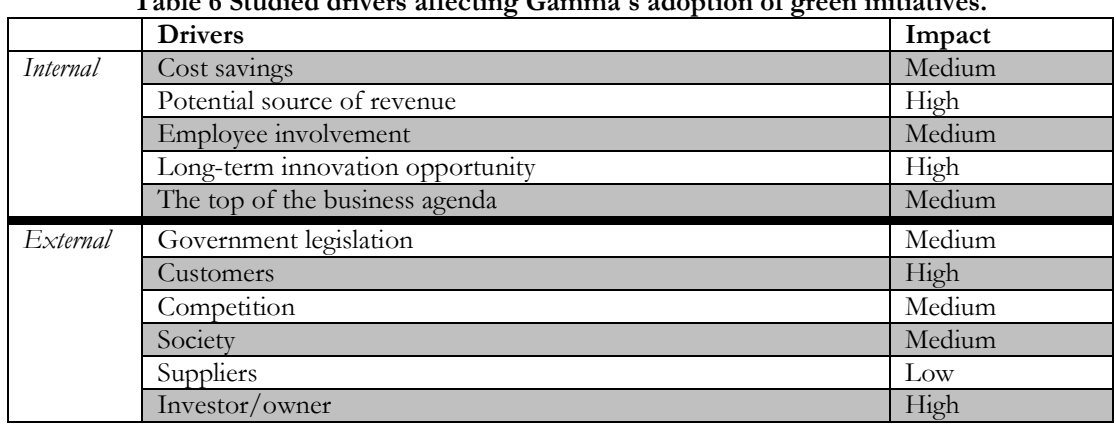

\section{Internal driving forces}

Cost savings are not seen as a major driver for Gamma. Potential source of revenue; Gamma aims to adopt green initiatives in order to increase the factor "value for money" for customers. This is done in order to increase sales as well as attract new customers. Gamma views the adoption of green initiatives as a revenue opportunity. Employee involvement; the employees within the company are involved to some extent but are not 
regarded a major driver. Long-term innovation opportunity; Gamma wants to be considered as a company on the cutting edge and works a lot to in order to achieve this. The top of the business agenda; this driver is not considered as significant at the moment.

\section{Other internal driving forces}

Gamma wants to be product unique in order to avoid price pressures and has therefore started to monitor and follow up its work with green initiatives.

\section{External driving forces}

Government legislation; there are already some government requirements regarding green initiatives, but Gamma wants to be prepared if green requirements and targets increase in the future. Customers are considered to be a significant driver and Gamma has conducted a survey in which the interest of $\mathrm{CO}_{2}$ reporting among its customers was investigated. It turned out that interest is especially high among public authorities and companies which have an aggressive sustainability profile. Competition; there are some competitors who have started to adopt green initiatives, but according to Gamma, the overall picture is a bit disjointed. It is not an easy task and Gamma is also somewhat uncertain how to proceed further. Suppliers; are not considered a driver since there is no pressure at all from the company's suppliers/partners. Investor/owners; Gamma has recently started to feel increased pressure from its owners, who have begun to shown interest and awareness of green initiatives.

\section{Other external driving forces}

Gamma also mentions that political requirements are an additional driver, since the company finds it interesting to follow what happens and if such requirements will increase in the future.

\section{Barriers for the adoption of green initiatives}

The impact of each of the studied barriers is shown in Table 7, followed by a description of how way these barriers affect Gamma's adoption of green initiatives.

Table 7 Studied barriers affecting Gamma's adoption of green initiatives.

\begin{tabular}{|c|c|c|}
\hline & Barriers & Impact \\
\hline \multirow[t]{5}{*}{ Internal } & Financial and economic & Medium \\
\hline & Technical & High \\
\hline & Information & High \\
\hline & Managerial & High \\
\hline & Organizational & Medium \\
\hline \multirow[t]{2}{*}{ External } & Policy & Low \\
\hline & Market & Medium \\
\hline
\end{tabular}

\section{Internal barriers}

Financial; Gamma has not heavily invested in green initiatives so far, but the situation may change if decisions are taken to replace its vehicles fleet with more environmental friendly alternatives. This would probably be a progressive process. Technical; the company has met some technical problems especially when it comes to the monitoring of $\mathrm{CO}_{2}$ emissions from its transports. Information; this is a significant barrier and Gamma has worked a lot with this. A lot of effort has been put into explaining the figures from the monitoring and estimations of $\mathrm{CO}_{2}$ emissions, since people have no understanding of whether these are 
low or high. Overall, it is difficult to really understand the impact of sustainability impact. Gamma states that it is a very subjective process, even if the company tries to be as objective as possible. There is a fear of scaring customers off. However, the company sees a tendency among its partners that they have successively started to accept green initiatives. The partners have identified advantages with green initiatives such as attracting new customers and being less vulnerable to price competition with their competitors. However, Gamma states that the level of knowledge regarding green initiatives is not much higher among customers. The representative of Gamma stresses that the resistance to change regarding green initiatives can be linked to the following argument:

"The transport industry is masculine and traditional"

Managerial; it has not been an easy task to explain to the top management why adopting of green initiatives is needed and why it matters. The interest and desire to adopt green initiatives have been pushed from a bottom-up direction. However, this subject received more attention after Gamma presented its strategy to become "product unique" and showed the results from the survey it had conducted. Without the strategy and the customer survey, it would probably have been difficult to persuade the top management. Organisational; since Gamma recently started to adopt green initiatives, employees have not been affected to any great extent. This may change in the future, especially when it come to the green initiative of eco-driving. However, Gamma stresses that as long as there are incentives to save money, and when the benefits of the change become clear, there will not be any problem.

\section{External barriers}

Policy is not seen as barrier and when it come to the market barriers Gamma mentions that there just a few customers that have showed interest and expressed some green requirements.

\section{Other barriers}

The economic situation could be viewed as a barrier. Gamma claims that if a company has difficulties with its economic situation, it will probably not prioritize the adoption of green initiatives. The representative of Gamma states the following:

"We must be trustworthy, we cannot offer services if we cannot fulfil them. It is better to be a little bit more cautions in our statements and then actually keep them."

\section{Green service offering and its pricing}

Gamma has no specific green service offering today. Customers turn to Gamma in order to get express deliveries and Gamma tries to do this in the wisest and most environmentally way possible. However, the company offers its customers $\mathrm{CO}_{2}$ declarations but this service offering is not categorised as a green service offering but is part of its overall service offering. The background to the adoption of the $\mathrm{CO}_{2}$ 
declaration is a customer survey. The result of the survey showed that the factor "value for money" was ranked lowest. Since Gamma is the price leader on the market and customers experience the company as an expensive alternative, it started to include added services into their overall service offerings in order to raise the factor "value for money". Furthermore, Gamma stresses that the company has no intention to develop a green service offering or product, and the Gamma representative points out:

\section{"I find it hard to believe that we will have a green profile"}

Since Gamma wants to raise the factor "value for money", they will not charge for the offered $\mathrm{CO}_{2}$ declarations which instead will be a part of the overall service offerings. Hence, Gamma states that the investment costs connected to the $\mathrm{CO}_{2}$ declarations have not been especially high since the company already possess a lot of transportation information in its existing IT-systems, and that these have been used in order to compile the $\mathrm{CO}_{2}$ declarations. Furthermore, the company states that it has improved the use of its IT-systems.

Even if sustainability is an important factor, the company still has to fulfil its requirements for the factors speed and reliability. One problem that may occur when it comes to not charging for $\mathrm{CO}_{2}$ reports is the fact that they may be then perceived to lack value. Due to the fact that Gamma has chosen the initial strategy of not charging $\mathrm{CO}_{2}$ declarations, it probably would give an ambiguous picture if the company in the future starts to charge for these reports. Gamma does not think that its competitors possess a similar information base and since Gamma is the price leader on the market, certain services should be included in the service offerings if customers chose to buy from them. However, Gamma cannot prevent its partners from charging for the $\mathrm{CO}_{2}$ declarations and the company has not actively informed its partners not to charge for this service offering. Gamma believes that the chosen strategy of not charging for green initiatives can be a way not only to attract new customers but also to raise the factor "value for money" for existing customers.

\section{DELTA}

Delta is part of a company active in the postal and logistics industry, and has the Nordic market as its geographical area. The company consists of nine business areas, of which Delta is one. Delta specialises in express and delivery transport and the turnover in 2009 was approximately 50.7 million EURO. The total number of employees is about 158 and approximately one of these employees work full-time with green initiatives. Delta consists of four different sub-areas Delivery, Distribution, Express (QuickPack) and Home Delivery.

\section{Service offerings and customers}

The range of service offerings offered by the four sub-areas (mentioned above) presents below;

- Delivery; offer flexible, fast and efficient deliveries to all local transportation needs. 
- Distribution; offer distribution services for planned and routine deliveries and routes.

- Express (QuickPack); fast deliveries within Sweden or all over the world. Can offer different kinds of transportation modes such as air, road or track.

- Home delivery; specialist in efficient home deliveries to consumers in all the Nordic countries.

Delta works mostly business to business, even though in some cases the company delivers direct to private consumers, especially in the sub-area Home deliveries.

\section{Drivers for the adoption of green initiatives}

Delta's perception of the impact of each driver studied is shown in Table 8, followed by a description of how these drivers affect the adoption of green initiatives.

Table 8 Studied drivers affecting Delta's adoption of green initiatives.

\begin{tabular}{|c|c|c|}
\hline & Drivers & Impact \\
\hline \multirow[t]{5}{*}{ Internal } & Cost savings & Medium \\
\hline & Potential source of revenue & High \\
\hline & Employee involvement & High \\
\hline & Long-term innovation opportunity & High \\
\hline & The top of the business agenda & High \\
\hline \multirow[t]{6}{*}{ External } & Government legislation & Medium \\
\hline & Customers & High \\
\hline & Competition & High \\
\hline & Society & High \\
\hline & Suppliers & Low \\
\hline & Investor/owner & High \\
\hline
\end{tabular}

\section{Internal driving forces}

Cost savings; overall it is desirable to achieve cost reductions by choosing to adopt green initiatives. However, Delta does not adopt green initiatives just to save money but instead cost reductions are viewed as a positive side effect. Potential source of revenue; one of the reasons why Delta has started to adopt green initiatives is because that the company wants to enhance their brand. Furthermore, Delta has the ambition to be viewed as a long-term sustainable company. The company wants the green approach to be a part of their profile. This will hopefully in turn lead to new market shares/customers. Delta believes that it would have lost customers if it had not started to invest and adopt green initiatives. Employee involvement; employees are partly viewed as a driver, but pressures mostly come from higher levels, since it is important for Delta to be viewed as an attractive workplace. Long-term innovation opportunity; from a long-term perspective the adoption of green initiatives is really important for Delta, because the company wants to be viewed as a sustainable company in the future. The top of the business agenda; Delta perceives itself better at investing and adopting green initiatives from a short-term rather than a long-term perspective. The company considers itself to be flexible and agile when it comes to meeting different kinds of problems from customers, as long as the results are profitable. This driver is therefore important due to the fact that it is linked to the business with customers. 


\section{Other internal drivers}

Delta wants to be viewed as an attractive workplace and aims to create a good working climate. The company finds it important to show its employees that green initiatives are an important issue. Delta has quite a lot of young employees, who also find this important, and the company offers all of its employees' environmental education in the form of an environmental "driving license". Furthermore, the company's drivers also receive training in eco-driving.

\section{External drivers}

Customers; Delta believes that the customers are its best driver and really appreciates demanding customers. But there are also customers that do not make any green requirements still Delta feels that customers today are more demanding regarding green initiatives than previously. The customers are perceived as important drivers and the representative of Delta states:

\section{"We must work together with our customers in order} to get the best effect."

Competition; Delta believes they have come further than their competitors with regard to the adoption of green initiatives and the company wants to maintain that lead. Therefore, Delta tries to benchmark its competitors' situation and how they are adopting green initiatives. Society; is seen as an important driver. Delta does not want to have bad publicity from social media because the company does not want to risk destroying its brand. Suppliers; suppliers are not perceived as a great driver even if it sometimes happens that suppliers make demands. Instead, Delta has the more active role when it comes to pushing its suppliers even they work together. Investor/owner; the owners put pressure on Delta and have set a target to reduce 30\% $\mathrm{CO}_{2}$ by 2015.

\section{Other external drivers}

Delta finds it important to work with green initiatives also from an ethic perspective. The company does not want to underestimate the importance of environmental concerns and instead strives to ensure that the impression they give is positive.

\section{Barriers for the adoption of green initiatives}

The impact of each of the barriers studied is shown in Table 9, followed by a description of the way in which these barriers affect Delta's adoption of green initiatives.

Table 9 Studied barriers affecting Delta's adoption of green initiatives.

\begin{tabular}{|c|c|c|}
\hline & Barriers & Impact \\
\hline \multirow[t]{5}{*}{ Internal } & Financial and economic & Medium \\
\hline & Technical & High \\
\hline & Information & High \\
\hline & Managerial & Low \\
\hline & Organizational & Low \\
\hline \multirow[t]{2}{*}{ External } & Policy & High \\
\hline & Market & Low \\
\hline
\end{tabular}




\section{Internal barriers}

Financial; Delta does not find it difficult to justify investments connected to the adoption of green initiatives but points out that when it comes to green investments it is more a question of finding smart solutions. Technical; the biggest problem when it comes to buying more environmental friendly vehicles, is the limited number of these on the market. They are hard to get and another problem is the limited gas stations where these vehicles can be refuelled. Delta also tries to measure carbon emissions, but does not find it an easy task. Information; the company has some problems with IT-support and its information systems, for example when it comes to $\mathrm{CO}_{2}$ reporting. Delta has customers that demand $\mathrm{CO}_{2}$ reporting. Managerial; this factor is not seen as a barrier as the company receives good support from the board of directors. Organizational; some employees cannot see the advantages of adopting green initiatives, although Delta does not see the organizational factor to be a barrier.

\section{Other barriers}

The company states that there are some problems with the legislation due to the fact that they cannot always buy the cars/vehicles it wants to because of the difficulties with refuelling.

\section{External barriers}

Policy; some laws and policies require a lot of paper work which takes a lot of time and leads to extra difficulties. Market; Delta states that it has suggested service offerings that had a positive effect on the environment but that customers have not been mature for these kinds of service offerings. Despite this, Delta does not see the market as a main barrier.

\section{Green service offering and its pricing}

Delta does not make a concrete green service offering. This is something that has been discussed, but Delta does not believe it can offer a green core product. Instead of marketing a concrete green service offering, the company has focused and worked with the brand in order to make it greener and more valuable for customers. On its homepage Delta wants to communicate that the company is a green brand/alternative and also suggests what kinds of positive green initiatives can be made within Delta. Examples can be more environmentally friendly transport alternatives, effective coordination of transports, bicycle messengers, more environmentally friendly transportation modes, education (e.g. eco driving and internal environmental training) and traffic safety.

When it comes to adoption of green initiatives, Delta believes that the first thing the company has to do is to start communicate the company's environmental policy and after that, gradually do more. Delta stresses that the company wants to be more confident before it starts to promote green service offerings. The company does not want to launch a green service offering and then not do anything about it. Delta wants to start to build up trust, both within the company and with their customers in order to be trustworthy.

Delta does not want to take charge of the green issues on its own but instead includes this in the agreement with the customers. However, the company has discussed this issue. Although Delta has received payment, for example from the county council that has 
requirements for biogas cars. But generally, Delta stresses that the margins are not big enough to take extra charge for the environment that instead must find smarter solutions. Delta does not belong to the group of companies that thinks that customers make a lot of demands but are not willing to pay for them. Delta believes that it is better to find and keep new customers than charging more. The company wants to work more closely with customers in different kinds of project in order to find alternative solutions together, which will hopefully lead to a stronger cooperation and a better and a more trustful relationship. Delta does not believe that companies have to invent a lot of green service offerings and charge extra for them. Instead it is better to increase sales. The company is aware of the fact that there can be situations when Delta has to charge for green initiatives e.g. investments of environmental friendly vehicles, but the idea of charging more for green services is something Delta neither supports nor believes in.

\section{EPSILON}

Epsilon is part of one of the world's leading logistics group. The company had a turnover of 530 million EURO in 2009 and has today approximately 4500 employees. Overall, approximately four employees work full-time concerning environmental sustainability in Sweden. The company is divided into four business units, which are presented below.

\section{Service offerings and customers}

Express; offers courier and express services to both businesses and end consumers.

Supply Chain; offers services such as customised solutions, inventory management, order fulfilment and transport.

Global Forwarding; focuses on air and ocean freight. It also offers customised solutions for industrial projects, customer management programs (CPM) and customs house brokerage.

Freight; offers flexible and customised services such as part loads and full loads by road, rail or intermodal transportation. It also offers trade fair services.

\section{Drivers for the adoption of green initiatives}

The impact of each of the drivers studied is illustrated in Table 10, followed by a description of how these affect Epsilon's adoption of green initiatives.

Table 10 Studied drivers affecting Epsilon's adoption of green initiatives.

\begin{tabular}{|l|l|l|}
\hline \multirow{4}{*}{ Internal } & Drivers & Impact \\
\cline { 2 - 3 } & Cost savings & High \\
\cline { 2 - 3 } & Potential source of revenue & Medium \\
\cline { 2 - 3 } & Employee involvement & High \\
\cline { 2 - 3 } & Long-term innovation opportunity & High \\
\cline { 2 - 3 } & The top of the business agenda & High \\
\hline \multirow{3}{*}{ External } & Government legislation & Low \\
\cline { 2 - 3 } & Customers & High \\
\cline { 2 - 3 } & Competition & High \\
\cline { 2 - 3 } & Society & Medium \\
\cline { 2 - 3 } & Suppliers & Low \\
\cline { 2 - 3 } & Investor/owner & High \\
\hline
\end{tabular}




\section{Internal drivers}

Cost savings; this varies between the different business units within Epsilon but the cost factor is a big driver. Potential source of revenue; this is at the moment not a major driver. Employee involvement; Epsilon measures this factor. A questionnaire is sent out every year to all employees and the questionnaire is reprimanded if the company average is too low. The average of Sweden is generally low. Epsilon tries to talk about sustainability issues more with employees and to communicate better. A long-term innovation opportunity; this is important for the business unit which the Epsilon representative is responsible over but the other business areas within Epsilon show a somewhat lower interest in this driver. Every business areas within Epsilon is not particular driving which can be explained by different business cultures. The top of the business agenda; this driver is also important for the business area for which the representative is responsible but interest among the other business areas is not very high. The Epsilon representative states that its business area tries to see how everything is affected from a broad perspective and feels a great pressure from customers.

\section{Other internal drivers}

The transport industry is a big part of the carbon dioxide problem; therefore Epsilon feels that it has to take responsibility for this. This is a hot topic; emissions have increased and are therefore seen as an internal driver within the company.

\section{External driver}

Government legislation; is not seen as a major driver because there are not very many legal requirements for the transport industry. Epsilon states that if it was a requirement to make annual reports and audit reports the situation might be different. Customer; is a major driver. Epsilon wants to be ready and available when and if customers start demanding green initiatives. Competition; the company has a vision to be an "Industry shaper" and this vision includes also sustainability issues. Market shares are seen as very important. Society; this is not a major driver and Epsilon does not receive much media attention. Suppliers; the suppliers do not take any green initiatives but they are very keen to meet Epsilon's requirements. Epsilon sees opportunities for cooperation with its suppliers, but this cooperation has not really started yet. Investor/owner; this driver has increased. It has been stated from the board that sustainability is an important matter and also that it is important to reach the set targets and goals.

\section{Other external drivers}

New market shares are an important driver for Epsilon. The company wants to win new customers by providing green initiatives. Research is another significant driver; Epsilon follows what is happening in research, at least in Sweden.

\section{Barriers for the adoption of green initiatives}

The impact of each of the barriers studied is shown in Table 11, followed by a description of how these barriers affect Epsilon's adoption of green initiatives. 
Table 11 Studied barriers affecting Epsilon's adoption of green initiatives.

\begin{tabular}{|l|l|l|}
\hline & Barriers & Impact \\
\hline \multirow{3}{*}{ Internal } & Financial and economic & High \\
\cline { 2 - 3 } & Technical & High \\
\cline { 2 - 3 } & Information & High \\
\cline { 2 - 3 } & Managerial & Low \\
\cline { 2 - 3 } & Organizational & Medium \\
\hline \multirow{2}{*}{ External } & Policy & Low \\
\cline { 2 - 3 } & Market & High \\
\hline
\end{tabular}

\section{Internal barriers}

Financial and economic; is a big issue. For example, if a business area does not show profit, the existence of green service offerings and why these service offerings do not show more profit can be queried. This in turn can lead to difficulties in justifying investment in any further adoption of green initiatives. Another contributing factor is that the transport business is a low margin industry. Technical; the technical development is not as fast as Epsilon would like. Information; Epsilon has IT-systems but the existing systems are not flexible enough, at least from the perspective of sustainability. However, Epsilon is currently working and investing in these systems in order to overcome this information barrier. Managerial issues; is not a seen as a barrier, Epsilon has good support from the top management. Organisational; Epsilon has high goals regarding the company's green future but this process are restricted by the time factor. Everyone knows that adoption of green initiatives is needed and is important but the interest among employees regarding these initiatives varies.

\section{External barrier}

Policy; is not seen as a barrier since Epsilon perceives there is a lack of political requirements regarding green initiatives. Market; this factor is seen as a barrier particularly when it comes to customers' lack of interest. The representative of Epsilon states the following:

"Customers just want to listen and hear that we are working with green initiatives but do not want to be a part of it. We need customers that are willing to do something as well."

\section{Other barriers}

Global functions that work with sustainability issues are considered to be as a barrier because there are different methods and approaches. This is a problem within large companies. Other problems are how information is handled and cultural differences. Different countries have different values and approaches while Sweden tries to look at sustainability on a global level.

\section{Green service offering and its pricing}

Not all business areas within Epsilon provide $\mathrm{CO}_{2}$ declarations, for example, one of the business areas does not offer these declarations due to lack of customers interest. Epsilon mentions other service offerings which may reduce the carbon dioxide even if this is not 
the main purpose of the service offering. Furthermore, the company also offers carbonoffset service offerings. Epsilon tries to communicate these service offerings in different ways to its customers but has noticed a problem. The sales people feel more comfortable about offering and selling $\mathrm{CO}_{2}$ declarations than the other green service offerings.

When it comes to $\mathrm{CO}_{2}$ declarations, Epsilon has a first draft of a pricing model. Customers get the annual report free but if they want more regular follow-ups, they have to pay extra. The interest in this service offering is low, but Epsilon hopes that this will increase in the future. The pricing model for $\mathrm{CO}_{2}$ declarations is just a test and this service offering may eventually be free. But if this service becomes more comprehensive, Epsilon wants to charge extra for it. In the case of the carbon-offset offering, the pricing of this service offering differs among the different business areas. Overall, pricing models for regular services are complicated and hard to understand, not only for customers but also for Epsilon. Many different costs are added to the price and the pricing is often done in different ways. However, Epsilon has a special tool where customers can fill in their own data, such as destination and additional costs. It is possible to choose between different transport options and it is easy to understand. In the future, Epsilon wants to integrate sustainability aspects of the offering into the tool as well. Not only will customers then become more aware of the sustainability impacts of their transports, but it could make it easier for Epsilon to communicate its sustainability work with its customers, something which is lacking today.

In the future, Epsilon thinks it is important to have transparency to show what its customers actually pay for. Epsilon tries to observe its competitors and how they work with the pricing of sustainability aspects, but the company does not really have a good benchmark process for this. The company does not want to take a too low or high price when for example it comes to consulting services. Epsilon wants to make money from sustainability but not too much. But it is difficult to know how others competitors price their services. Epsilon considers itself to be well advanced in its green service offerings but is poor at communicating these services. The company hopes to overcome this problem with a new tool on the website. Epsilon wants to start to show its customers what the company has to offer.

Epsilon faces another barrier when it comes to customers, who prefer to go to a consulting firm that has sustainability expertise instead of using Epsilon, when they implement green initiatives. Customers are probably a little bit sceptical about the fact that Epsilon has started to offer additional service offerings, for example consulting services. Epsilon finds rather hard to market these kinds of services and to find a good pricing model. Epsilon has a lot of knowledge and information about its network and is able to make transport optimisations, but customers may question why these additional services have not been done before.

\section{ZETA}

Zeta is part of one of the world's biggest logistics company. The company provides land transport within Europe, global ocean- and airfreight and customised logistics solutions. Zeta Sweden (hereafter labelled Zeta) had a turnover of approximately 1248 million euro in 2009. The company employs approximately 4000 people and 6000 other people are 
included together with cooperative road carriers. Only one person works full-time with sustainability issues at Zeta but there are approximately 5-6 full-time jobs in this area if all the work done by different individuals is actually put together.

\section{Service offerings and customers}

Zeta offers everything from rail transport to packet transport. Rail transport includes in turn wagonload and combined transport. The company offers also all types of truckloads of palletized goods, and in some cases, long goods. Furthermore, Zeta offers logistics services such as warehousing and the handling of goods. Zeta does not offer courier services or mail freight. Zeta offers also consulting services such as transportation optimization, simulation and development of logistics (linked to the environment). This area is linked to green initiatives in terms of emissions calculations and project management in order to help customers develop and improve their environmental offerings. The split between inputs for industry and consumer products is about $50 \%$ each.

\section{Drivers for the adoption of green initiatives}

The impact of the drivers studied is illustrated in Table 12, followed by a description of how these affect Zeta's adoption of green initiatives.

Table 12 Studied drivers affecting Zeta's adoption of green initiatives.

\begin{tabular}{|c|c|c|}
\hline & Drivers & Impact \\
\hline \multirow[t]{5}{*}{ Internal } & Cost savings & High \\
\hline & Potential source of revenue & High \\
\hline & Employee involvement & High \\
\hline & Long-term innovation opportunity & High \\
\hline & The top of the business agenda & Medium \\
\hline \multirow[t]{6}{*}{ External } & Government legislation & High \\
\hline & Customers & High \\
\hline & Competition & Medium \\
\hline & Society & Medium \\
\hline & Suppliers & Low \\
\hline & Investor/owner & Medium \\
\hline
\end{tabular}

\section{Internal driving forces}

Cost savings; as a forwarder Zeta might not be able to make large cost savings but instead the focus should be on working to ensure its customers reduce their costs. Otherwise, the company risks losing market shares to its competitors. It is important to be prepared for this in the future. Potential source of revenue; Zeta's incentive to adopt green initiatives is to win new customers. Furthermore, it can also be a way to retain existing customers. However, the company sees business potential here and hopes that green initiatives will become more important and that customers will be willing to pay for them. Employee involvement; this driver is very important. The employees of the company are involved in the adoption of green initiatives. Zeta works to profile the entire company as a green logistics service provider instead of just offering green service offerings. Long-term innovation opportunity; the company views green initiatives from a long-term perspective because it is stated from the top that green initiatives should not be viewed from a shortterm perspective. The top of the business agenda; there is not much focus on this driver at the moment because the company has some concerns about which direction it should take regarding green initiatives. 


\section{Other internal drivers}

When it comes to adopting green initiatives, Zeta stresses the need for a responsible green unit as well as for support, engagement and feedback from the highest levels within the company.

\section{External drivers}

Government legislation; has definitely a major impact, but Zeta does not perceive that there is so much legislation regarding green initiatives. Customers; this is difficult, because customers have different requirements which is not always possible to fulfil. Big customers have always some kinds of sustainability requirements such as ISO certifications or special kinds of transport modes, while requirements from small customers are not vey clear. Competition; Zeta does not sense any competition from its competitors and considers itself to know what its competitors are doing in this field. Zeta does not think that any customers choose Zeta or one of its competitors just because of the adoption of green initiatives. This may change in the future and to adopt green initiatives may even become a competitive advantage. Society; Zeta aims of course to be a good company both for its employees and the community. The company perceives that it is doing enough but that it can be better. Suppliers are not really seen as a driver at all. Perhaps they should become more involved because if Zeta improves its adoption of green initiatives, this would probably generate more income for its suppliers. Investors/owners; there are some requirements from the owners, but they are not seen as a major driver.

\section{Barriers for the adoption of green initiatives}

The impact of the barriers studied is illustrated in Table 13, followed by a description of how these affect Zeta's adoption of green initiatives.

Table 13 Studied barriers affecting Zeta's adoption of green initiatives.

\begin{tabular}{|l|l|l|}
\hline & Barriers & Impact \\
\hline \multirow{3}{*}{ Internal } & Financial and economic & High \\
\cline { 2 - 3 } & Technical & Low \\
\cline { 2 - 3 } & Information & High \\
\cline { 2 - 3 } & Managerial & Low \\
\cline { 2 - 3 } & Organizational & High \\
\hline \multirow{2}{*}{ External } & Policy & High \\
\cline { 2 - 3 } & Market & High \\
\hline
\end{tabular}

\section{Internal barriers}

Financial; this factor is perhaps not the biggest barrier but still has a strong impact. Technical; is not one of the major barriers. Information is normally not a problem, but while Zeta has a large customer base and no standardised green service offering, information can be a problem. In addition, the company does not perceive that its IT-system is supportive enough, especially with regard to estimating emissions. Managerial issues; are not seen as a barrier. Organisation; Zeta has some problems when it comes to different functions within the company. The views on how to adopt and work with green initiatives vary within the company because each function has its own aims and ways of working with these. 


\section{External barriers}

Policy; Zeta does not really agree with the different green requirements which means that their acceptance is not fully supported within the company. Market; this is a major barrier since customers have so many different requirements. There are as many requirements as there are customers. There is some customer consensus about the requirements but the different nuances among these requirements cause problem. Zeta stresses that trade associations can do more to reduce these market barriers.

\section{Green service offering and its pricing}

Zeta does not have any developed green service offering but instead the company profiles its network as a freight collective network. The company offers customised green solutions to customers who demand this. Zeta tries to target customers interested in these kinds of offerings, for example the automotive industry. Other customers, who are interested in green service offerings, are those who work with consumer products and also food industry, which is interested in environmental and emissions calculations and the use of more environmental friendly vehicles. The company perceives that it answers customers' requirements quite well and promotes thoughts and ideas for improvements proposal certain selected customers. Zeta has a steady steam of environmentally sustainable projects, which indicates that this is a significant part of the development of logistics.

Zeta does not work with different price models for green initiatives Instead, the environmental aspect is added as an extra cost. For example, if Zeta invests in green equipment, this cost is included in the transport calculation. The representative of Zeta states:

\section{"How do you charge for a more environmentally sustainable transport solutions? We would like to work more with value- based pricing to our customers but the problem is what is it worth to customers?"}

As mentioned above, if Zeta has additional costs because the solution has a more environmentally sustainable orientation, these costs are integrated into the service calculation. These additional costs are not usually value-priced but are instead more costpriced. This method is used because the company finds it difficult to estimate the perceived value for its customers. One reason for this problem is that there is no real market pricing regarding these kinds of service offerings. Zeta perceives that its customers are not willing to pay more for green initiatives although there are customers who want to pay for more environment friendly vehicles. Zeta has discussed the advantages and disadvantages of a more value-based pricing system for green initiatives, and the company has also conducted market research among its customers on this issue. The market research showed that customers will be willing to pay more for the environment in the future but not today. One reason for this could be that are no requirements for this today and also the fact that customers do not want to pay for something that nobody else does. Instead, the customers only experience a loss of competitiveness. The customers require information about their transports and think that 
$\mathrm{CO}_{2}$ declaration should be included in the service offering. Zeta feels that it cannot charge for this and instead, $\mathrm{CO}_{2}$ declarations are seen as added value to its service offerings. Furthermore, Zeta has observed that its customers are not interested in green initiatives but that their customers in turn are interested and are willing to pay for these. Zeta stresses that there are a lot of green opportunities but that there is no money for implementation, especially when customers are not willing to invest as well. The company is met with suspicion from customers when Zeta suggests that they should split the investments costs. Instead the customers think that Zeta can take the entire investment alone.

As mentioned earlier, at the moment pricing models, which take green initiatives into account, do not exist within the company. Zeta finds this issue interesting but it is difficult to evaluate green initiatives and reach customers with this message. Perhaps government legislation could facilitate this problem and Zeta believes that tougher regulations, standards and monitoring could facilitate the process. 
APPENDIX 3

\section{The QuestionnaIRE}





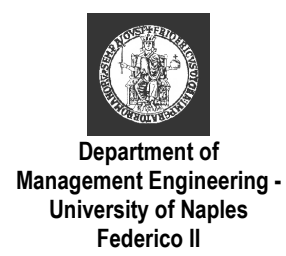

\section{SURVEY ON GREEN SUPPLY CHAIN INITIATIVES IN THE TRANSPORT AND LOGISTICS SERVICE INDUSTRY}

\section{Informant title:}

Entrepreneur or main shareholder or company owner

Chief Executive Officer

Logistics/supply chain manager

Environmental sustainability manager

Other (please specify)

\section{SECTION 1 - COMPANY PROFILE}

1.1 - Can you please indicate, among the following bands, the number of employees in the year 2010? (If your company belong to a corporate group, please answer to the unit/subsidiary in which you operate)
$1-9$
$10-49$
$50-99$
$100-249$
$250-499$
$\geq 500$

1.2 - What has been the annual company turnover in the year 2010? (If your company belongs to a corporate group, please answer to the unit/subsidiary in which you operate)
$<2 \mathrm{M} €$
$2-10 \mathrm{M} €$
$10-20 \mathrm{M} €$
$20-50 \mathrm{M} €$
$50-100 \mathrm{M} €$
$>100 \mathrm{M} €$

1.3 - What is the geographical scale of your company's operations? (multiple choices are possible) Local Europe

Regional

Global

National 
1.4 - Which type of product results in the major contribution of your deliveries?

about $50 \%$ of each
$\square$
$\square \quad$ more than $50 \%$ consumer goods
$\square$
$\square$
$\square$ Don't know

1.5 - Which of the following ICT tools are used in your company? (multiple choices are possible)

\begin{tabular}{|l|c|l|c|}
\hline Electronic Data Interchange - EDI & $\square$ & Wireless LAN & $\square$ \\
\hline Global Positioning System - GPS & $\square$ & Radio Frequency Identification - RFID & $\square$ \\
\hline Bar code & $\square$ & Enterprise Resource Planning - ERP & $\square$ \\
\hline Radio frequency & $\square$ & Customer Relationship Management - CRM & $\square$ \\
\hline Local Area Network - LAN & $\square$ & Other (please specify): & $\square$ \\
\hline
\end{tabular}

1.6 - Please indicate the level of ICT integration with other supply chain participants (please rate where $1=$ Not integrated at all and $5=$ Fully integrated and automated)

\begin{tabular}{|l|c|c|c|c|c|c|}
\cline { 2 - 7 } \multicolumn{1}{c|}{} & $\mathbf{1}$ & $\mathbf{2}$ & $\mathbf{3}$ & $\mathbf{4}$ & $\mathbf{5}$ & Don't know \\
\hline Our customers & $\square$ & $\square$ & $\square$ & $\square$ & $\square$ & $\square$ \\
\hline Our customers' customers & $\square$ & $\square$ & $\square$ & $\square$ & $\square$ & $\square$ \\
\hline Suppliers of our customers & $\square$ & $\square$ & $\square$ & $\square$ & $\square$ & $\square$ \\
\hline Other logistics service providers & $\square$ & $\square$ & $\square$ & $\square$ & $\square$ & $\square$ \\
\hline
\end{tabular}

1.7 - What was the percentage of your turnover generated by each of the service categories listed below in the year 2010? (totally $100 \%$ )

\begin{tabular}{|c|c|c|c|c|c|c|c|}
\hline $\begin{array}{c}\text { Logistics } \\
\text { and transport } \\
\text { services } \\
\text { offered }\end{array}$ & ț & 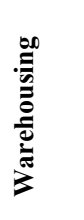 & 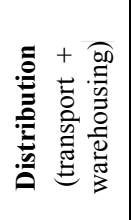 & 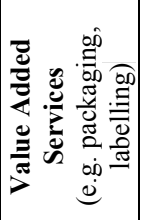 & 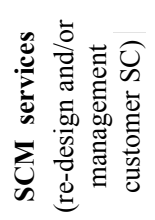 & 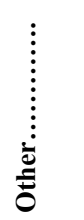 & 离 \\
\hline & 1 & 2 & 3 & 4 & 5 & 6 & $(1+2+3+4+5+6)$ \\
\hline 2010 & $\%$ & $\%$ & $\%$ & $\%$ & $\%$ & $\%$ & $100 \%$ \\
\hline
\end{tabular}

\section{SECTION 2 - ADOPTION OF GREEN INITIATIVES AND RESOURCES}

2.1 - Does your company have any environmental office or department responsible for green initiatives?

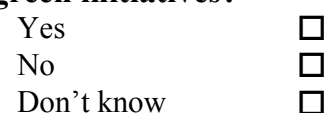

2.2 - Can you please indicate the percentage of full-time employees (or equivalent) on total company employees with environmental responsibilities or tasks? $\%$ 
2.3 - The environment is part of our company's overall business strategy:

Yes, formal/explicit

Yes, informal/implicit

No, it's not

$\square$ Don't know

2.4 - Environmental concerns are considered in a high extent when implementing changes in our company's logistics system: $(1=$ totally disagree and $5=$ totally agree $)$

$\begin{array}{cccccc}1 & 2 & 3 & 4 & 5 & \text { Don't know } \\ \square & \square & \square & \square & \square & \square\end{array}$

2.5 - Has your company currently adopted green logistics management initiatives?

Yes

No

Don't know

\section{ATTENTION}

If you answered YES to question 2.5, go ahead to point 2.6

If you answered "NO" or "DON'T KNOW" to question 2.5, go to SECTION 4

2.6 - For environmental reasons, our company has in a high extent adopted in the following transport related measures: $(1=$ totally disagree and $5=$ totally agree $)$

\begin{tabular}{|l|l|l|l|l|l|l|}
\hline & $\mathbf{1}$ & $\mathbf{2}$ & $\mathbf{3}$ & $\mathbf{4}$ & $\mathbf{5}$ & Don't know \\
\hline Alternative fuels for transports & & & & & & \\
\hline $\begin{array}{l}\text { Modifications to vehicle specifications/design (e.g. } \\
\text { aerodynamic features, low rolling resistances tires) }\end{array}$ & & & & & & \\
\hline Eco-driving & & & & & & \\
\hline Recurrent environmental pilot project & & & & & & \\
\hline Switch to less energy-intensive transports modes & & & & & & \\
\hline Greater use of intermodal transport & & & & & & \\
\hline Measures to improve vehicle loading & & & & & \\
\hline Measures to reduce empty running & & & & & \\
\hline Transport planning management (e.g. route optimization) & & & & & \\
\hline $\begin{array}{l}\text { Structural changes to your logistics system (e.g. number, size } \\
\text { or location of facilities) }\end{array}$ & & & & & \\
\hline Offer documented emissions data & & & & & & \\
\hline Other (please specify): & & & & & & \\
\hline
\end{tabular}

2.7 - For environmental reasons, our company has in a high extent adopted in the following supply chain related measures: ( $1=$ totally disagree and 5=totally agree)

\begin{tabular}{|c|c|c|c|c|c|c|}
\hline \multirow{2}{*}{ Renewable/green energy } & 1 & 2 & 3 & 4 & 5 & Don't know \\
\hline & & & & & & \\
\hline \multicolumn{7}{|l|}{ Collecting information on energy use/carbon footprint } \\
\hline \multicolumn{7}{|l|}{ Reduction in the amount of packaging } \\
\hline \multicolumn{7}{|l|}{ Increase the amount of waste recycled } \\
\hline \multicolumn{7}{|l|}{ Environmental certification (e.g. ISO 14001) } \\
\hline \multicolumn{7}{|l|}{ Environmental Management System (EMS) } \\
\hline \multicolumn{7}{|l|}{ Emission off-set programmes } \\
\hline \multicolumn{7}{|l|}{ Setting a corporate GHG emission reduction target } \\
\hline $\begin{array}{l}\text { Cooperation with our suppliers/partners in order to reach } \\
\text { environmental targets }\end{array}$ & & & & & & \\
\hline
\end{tabular}




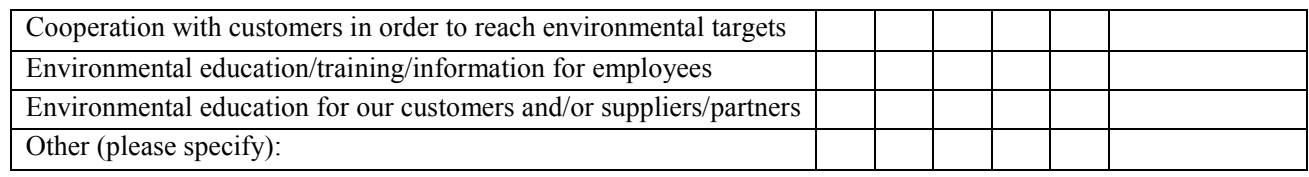

2.8 - The following ICT applications are strongly used in managing the environmental impact of our company's transport and logistics operations (please rate where $1=$ totally disagree and $5=$ totally agree)

\begin{tabular}{|l|l|l|l|l|l|l|}
\hline & $\mathbf{1}$ & $\mathbf{2}$ & $\mathbf{3}$ & $\mathbf{4}$ & $\mathbf{5}$ & Don't know \\
\hline Transport management systems - TMS & & & & & & \\
\hline Vehicle routing and scheduling & & & & & & \\
\hline Transport simulation/planning & & & & & & \\
\hline Tracking and tracing system & & & & & & \\
\hline Inventory management & & & & & & \\
\hline Warehouse management & & & & & & \\
\hline Network/facility location configuration & & & & & & \\
\hline Distribution planning & & & & & & \\
\hline Purchasing and supply planning & & & & & & \\
\hline Other (please specify): & & & & & & \\
\hline
\end{tabular}

2.9 - The following tracking \& tracing functionalities are strongly used in managing the environmental impact of our company's transport and logistics operations (please rate where $1=$ totally disagree and $5=$ totally agree)

\begin{tabular}{|l|l|l|l|l|l|l|}
\hline & $\mathbf{1}$ & $\mathbf{2}$ & $\mathbf{3}$ & $\mathbf{4}$ & $\mathbf{5}$ & Don't know \\
\hline Tracking of shipment documents & & & & & & \\
\hline Tracking of containers & & & & & & \\
\hline Tracking of pallets & & & & & & \\
\hline Tracking of packages & & & & & & \\
\hline Tracking of inventories & & & & & & \\
\hline Tracking of full truck load & & & & & & \\
\hline Tracking of and tracing is not used at all & & & & & & \\
\hline
\end{tabular}

2.10 - The following professional functions are involved in a high extent with our company's green supply chain initiatives: (please rate where $1=$ totally disagree and $5=$ totally agree)

\begin{tabular}{|l|l|l|l|l|l|l|}
\hline & $\mathbf{1}$ & $\mathbf{2}$ & $\mathbf{3}$ & $\mathbf{4}$ & $\mathbf{5}$ & Don't know \\
\hline Logistics/transport department & & & & & & \\
\hline Supply chain department & & & & & & \\
\hline Warehouse/terminals & & & & & & \\
\hline Environmental department & & & & & & \\
\hline Marketing department & & & & & & \\
\hline Sales department & & & & & & \\
\hline Top management & & & & & & \\
\hline IT-department & & & & & & \\
\hline Finance department & & & & & & \\
\hline Purchasing department & & & & & & \\
\hline Other (please specify): & & & & \\
\hline
\end{tabular}


2.11 - Does your company measure the performance of green supply chain initiatives that are adopted?

Yes

No

Don't know

\section{SECTION 3 - DRIVER OF GREEN LOGISTICS INITIATIVES}

3.1 - The following stakeholder group(s) exert strong influence when adopting environmental logistics initiatives in our company: (please rate where $1=$ totally disagree and $5=$ totally agree)

\begin{tabular}{|l|l|l|l|l|l|l|}
\hline & $\mathbf{1}$ & $\mathbf{2}$ & $\mathbf{3}$ & $\mathbf{4}$ & $\mathbf{5}$ & Don't know \\
\hline Competitors & & & & & & \\
\hline Customers & & & & & & \\
\hline Management & & & & & & \\
\hline Transport/Logistics Suppliers or partners & & & & & & \\
\hline Transport/Logistics Equipment Suppliers & & & & & & \\
\hline Employees & & & & & & \\
\hline Experts (academics/consultants) & & & & & & \\
\hline Trade bodies & & & & & & \\
\hline Government and public bodies & & & & & & \\
\hline Owners/shareholders & & & & & & \\
\hline Insurers & & & & & & \\
\hline Other (please specify): & & & & & & \\
\hline
\end{tabular}

3.2 - The following arguments have a strong impact on decisions to adopt green logistics/supply chain initiatives in our company: (please rate where $1=$ totally disagree and $5=$ totally agree)

\begin{tabular}{|l|l|l|l|l|l|l|}
\hline & $\mathbf{1}$ & $\mathbf{2}$ & $\mathbf{3}$ & $\mathbf{4}$ & $\mathbf{5}$ & Don't know \\
\hline Cost reductions for customers & & & & & & \\
\hline Cost reductions for suppliers/partners & & & & & & \\
\hline Cost reductions within my company & & & & & & \\
\hline Improve customer relationship & & & & & & \\
\hline Improve customer service & & & & & \\
\hline Increase firm's competiveness & & & & & & \\
\hline Increase firm's revenue & & & & & \\
\hline Increase ROI & & & & & \\
\hline Increase market shares & & & & & & \\
\hline National and EU laws and regulations & & & & & & \\
\hline Economic means of control (e.g. taxes, subventions) & & & & & & \\
\hline Improve brand image & & & & & & \\
\hline Reduce company risk & & & & & & \\
\hline Other (please specify): & & & & & & \\
\hline
\end{tabular}

3.3 - The following arguments have a strong impact on decisions to investing in ICT to support green initiatives in our company: (please rate where $1=$ totally disagree and $5=$ totally agree)

\begin{tabular}{|l|l|l|l|l|l|l|}
\hline & $\mathbf{1}$ & $\mathbf{2}$ & $\mathbf{3}$ & $\mathbf{4}$ & $\mathbf{5}$ & Don't know \\
\hline Reduce cost of transport and logistics operations & & & & & & \\
\hline Improve internal control on environmental performance & & & & & & \\
\hline Improve response to customer's green needs & & & & & & \\
\hline Improve overall quality of customer service & & & & & & \\
\hline Improve competitiveness & & & & & & \\
\hline
\end{tabular}


Improve service differentiation

Other (please specify)

\section{SECTION 4 - BARRIERS TO GREEN SUPPLY CHAIN INITIATIVES}

4.1 - The following internal elements are strong barriers when adopting green supply chain initiatives in our company: (please rate where $1=$ totally disagree and $5=$ totally agree)

\begin{tabular}{|c|c|c|c|c|c|c|}
\hline & 1 & 2 & 3 & 4 & 5 & Don't know \\
\hline \multicolumn{7}{|l|}{ High investments costs } \\
\hline \multicolumn{7}{|l|}{ Uncertain payback period } \\
\hline \multicolumn{7}{|c|}{ Lack of financial resources } \\
\hline \multicolumn{7}{|c|}{$\begin{array}{l}\text { Lack of organisational/human resources specifically } \\
\text { devoted to manage such initiatives }\end{array}$} \\
\hline \multicolumn{7}{|c|}{ Lack of knowledge/skills in-house } \\
\hline \multicolumn{7}{|l|}{ Lack of ICT skills internal } \\
\hline \multicolumn{7}{|l|}{ Lack of ICT skills external } \\
\hline \multicolumn{7}{|l|}{ High ICT running costs } \\
\hline Other (please specify): & & & & & & \\
\hline
\end{tabular}

4.2 - The following external elements are strong barriers when adopting green supply chain initiatives in our company: (please rate where $1=$ totally disagree and $5=$ totally agree)

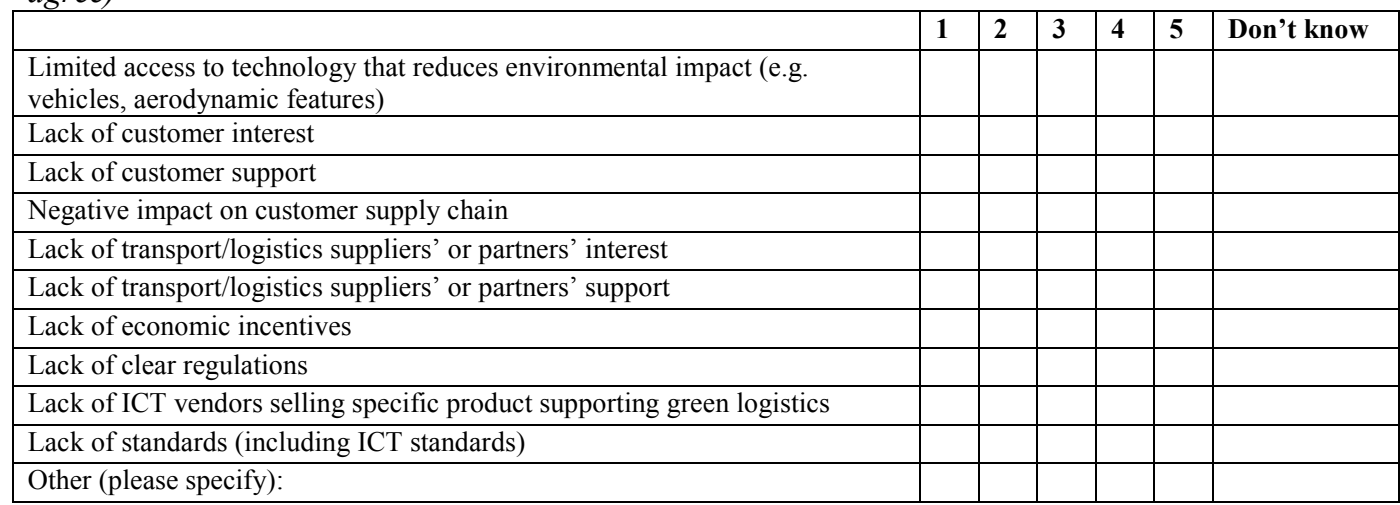

\section{SECTION 5 - FUTURE: ADOPTED GREEN SUPPLY CHAIN INITIATIVES WITHIN THREE YEARS}

5.1 - Within 3 years, our company plans in a high extent adopt the following transport related measures for environmental reasons: (please rate where $1=$ totally disagree and $5=$ totally agree)

\begin{tabular}{|c|c|c|c|c|c|c|}
\hline & 1 & 2 & 3 & 4 & 5 & Don't know \\
\hline \multicolumn{7}{|l|}{ Alternative fuels for transports } \\
\hline \multicolumn{7}{|l|}{$\begin{array}{l}\text { Modifications to vehicle specifications/design (e.g. } \\
\text { aerodynamic features, low rolling resistances tires) }\end{array}$} \\
\hline \multicolumn{7}{|l|}{ Eco-driving } \\
\hline \multicolumn{7}{|l|}{ Recurrent environmental pilot project } \\
\hline \multicolumn{7}{|l|}{ Switch to less energy-intensive transports modes } \\
\hline Greater use of intermodal transport & & & & & & \\
\hline
\end{tabular}




\begin{tabular}{|l|l|l|l|l|l|l|}
\hline Measures to improve vehicle loading & & & & & & \\
\hline Measures to reduce empty running & & & & & & \\
\hline Transport planning management (e.g. route optimization) & & & & & & \\
\hline $\begin{array}{l}\text { Structural changes to your logistics system (e.g. number, } \\
\text { size or location of facilities) }\end{array}$ & & & & & & \\
\hline Offer documented emissions data & & & & & & \\
\hline Other (please specify): & & & & & \\
\hline
\end{tabular}

\section{2 - Within 3 years, our company plans in a high extent to adopt the following supply} chain related measures for environmental reasons: (please rate where $1=$ totally disagree and $5=$ totally agree)

\begin{tabular}{|l|l|l|l|l|l|l|}
\hline & $\mathbf{1}$ & $\mathbf{2}$ & $\mathbf{3}$ & $\mathbf{4}$ & $\mathbf{5}$ & Don't know \\
\hline Renewable/green energy & & & & & & \\
\hline Collecting information on energy use/carbon footprint & & & & & & \\
\hline Reduction in the amount of packaging & & & & & & \\
\hline Increase the amount of waste recycled & & & & & & \\
\hline Environmental certification (e.g. ISO 14001) & & & & & & \\
\hline Environmental Management System (EMS) & & & & & \\
\hline Emission off-set programmes & & & & & & \\
\hline Setting a corporate GHG emission reduction target & & & & & & \\
\hline $\begin{array}{l}\text { Cooperation with your suppliers/partners in order to reach } \\
\text { environmental targets }\end{array}$ & & & & & & \\
\hline $\begin{array}{l}\text { Cooperation with customers in order to reach environmental } \\
\text { targets }\end{array}$ & & & & & & \\
\hline Environmental education/training/information for employees & & & & & & \\
\hline $\begin{array}{l}\text { Environmental education for your customers and/or } \\
\text { suppliers/partners }\end{array}$ & & & & & & \\
\hline Other (please specify): & & & \\
\hline
\end{tabular}





\section{Papers}

The articles associated with this thesis have been removed for copyright reasons. For more details about these see:

http://urn.kb.se/resolve?urn=urn:nbn:se:liu:diva-106783 